\title{
Métodos Restritos e Validação de Modelos Simétricos de Regressão
}

Francisco José de Azevêdo Cysneiros

\author{
Tese apresentada \\ ao \\ Instituto de Matemática e Estatística \\ da \\ Universidade de São Paulo \\ para \\ obtenção do grau \\ de \\ Doutor em Estatística
}

Área de Concentração: Estatística

Orientador: Prof. Dr. Gilberto Alvarenga Paula

São Paulo, 6 de fevereiro de 2004 


\title{
Métodos Restritos e Validação de \\ Modelos Simétricos de Regressão
}

Francisco José de Azevêdo Cysneiros

\begin{abstract}
Este exemplar corresponde à redação final da Tese devidamente corrigida e defendida por Francisco José de Azevêdo Cysneiros e aprovada pela comissão julgadora.
\end{abstract}

Aprovado em : 6 de fevereiro de 2004

Comissão julgadora:

- Prof. Dr. Gilberto Alvarenga Paula (Orientador)

IME/USP

- Prof. Dr. Heleno Bolfarine

IME/USP

- Prof ${ }^{a}$ Dr $\underline{\text { a }}$ Clarice Garcia Borges Demétrio

ESALQ/USP

- Prof. Dr. Filidor Edilfonso Vilca Labra IMECC/UNICAMP

- Prof. Dr. Gauss Moutinho Cordeiro UFRPE 
À minha mãe e irmãos, com gratidão,

Ao meu pai Gilberto (in memorian), com saudade,

À minha esposa

Audrey

com eterna paixão,

Ao meu filho

Rafael

com admiração,

dedico com carinho e amor. 


\section{Agradecimentos}

- Ao Professor Gilberto a confiança, o incentivo, as oportunidades oferecidas, e a excelente orientação dedicada na elaboração deste trabalho.

- À minha esposa Audrey, o amor e o apoio a mim concedido, em especial, ao meu filho, Rafael, a compreensão, o amor e o carinho por ele oferecido.

- Aos meu Pais, Gilberto e Gilvanete, que me forneceram princípios básicos e fundamentais para minha formação moral e dedicação integral ao meu objetivo.

- Aos meus irmãos Jorge e Beto, a eterna união.

- Aos meu sobrinhos e sobrinhas o carinho que sempre tive.

- Ao meu sogro Washington (in memorian), a minha sogra Lauricy, ao Seu Mariz (in memorian), as minhas cunhadas e cunhados a convivência alegre. Em especial a minha cunhada Lourdinha, a imensa ajuda nesses anos.

- Aos professores do Instituto de Matemática e Estatística que ajudaram na minha formação acadêmica.

- Ao Professor Dr. Manuel Galea pela sua contribuição nos resultados do Capítulo 4 desta Tese.

- Aos meus amigos que me apoiaram e ajudaram permitindo que este passo da minha vida fosse dado.

- Aos colegas do Departamento e Estatística da Universidade Federal da Pernambuco o apoio dado durante este curso.

- A Enivaldo Rocha, Jacira Guiro, Gauss Cordeiro, Francisco Cribari, Cláudia Lima e Manoel Senna que sempre me incentivaram nesta caminhada. - Aos amigos Paulo de Tarso, Maria Paula Chicarino, Raquel Valle, Érika Fukunaga, Márcia Branco, Iracema Arashiro, Jacqueline David, Regina Ishimoto, Carine Savalli, a agradável convivência e os momentos de descontração.

- À CAPES o apoio financeiro através do PICDT (Programa Institucional de Capacitação Docente e Técnica). 


\section{Resumo}

É conhecido, na literatura, que a modelagem sob a suposição de erros normalmente distribuídos pode ser altamente influenciada por observações extremas. O objetivo deste trabalho é apresentar alguns resultados na área de modelagem estatística de regressão com erros distribuídos na família simétrica, que contempla distribuições com caudas mais pesadas do que a normal. Numa primeira etapa, são apresentados alguns resultados na classe simétrica de distribuições. Em seguida, métodos de validação de modelos estatísticos baseados na teoria de influência local desenvolvida por Cook (1986) são apresentados. Quando a suposição de homoscedasticidade do modelo não é verificada, modelos heteroscedásticos são propostos em que a variância do modelo está relacionada, através de uma função de ligação, com um conjunto de variáveis explicativas. Métodos de validação são, também, desenvolvidos nesse caso e conjuntos de dados reais são utilizados para ilustrar a teoria proposta. Numa segunda etapa, discutimos a parte inferencial em modelos simétricos de regressão lineares com restrições nos parâmetros. Desenvolvemos processos iterativos para a estimação dos parâmetros e, também, alguns testes estatísticos, tais como razão de verossimilhanças, Wald e escore, para dois casos gerais de hipóteses restritas na forma de desigualdades lineares. Conjuntos de dados reais são utilizados para ilustrar a teoria desenvolvida. Rotinas computacionais originais em S-Plus e R para a obtenção das estimavas restritas e irrestritas em modelos simétricos lineares e não-lineares são desenvolvidas e apresentadas na web-page www. de.ufpe.br/ cysneiros/elliptical/elliptical.html. Focamos também modelos de regressão com erros $t$-Student para a análise de dados longitudinais com restrições nos parâmetros na forma de desigualdade lineares. 


\begin{abstract}
It is well known that statistical modelling under the assumption of errors normally distributed may be highly influenced by extreme observations. The objective of this work is to present some results in the area of regression models with errors distributed in the symmetrical class that contemplates distributions with heavier/lighter tails than the normal. In the first stage, some results in the symmetrical class of distributions are presented. Then, some diagnostic methods based on local influence are developed for linear and nonlinear symmetrical models as well as standardized residuals are proposed. When the assumption of homoscedasticity is not verified, heteroscedastic models are proposed. Here, the variance is related through a link function with a set of explanatory variables. Diagnostic procedures are also developed in this case. Real data sets are given to illustrate the proposed methods. In the second stage, symmetrical linear regression models with parameter contraints in linear inequality are discussed. Iterative process for the parameter estimation as well as some statistical tests, such as likelihood ratio, Wald and score, for two general cases of restricted hypotheses are given. Examples with real data are also used to illustrate the restricted methods. A group of original computational routines in S-Plus and $R$ for obtaining restricted and unrestricted estimates in symmetrical linear and nonlinear regression models are developed and presented in the web-page www.de.ufpe.br/ cysneiros/elliptical/elliptical.html. Finally, we focus on $t$-Student linear regression models to analyse longitudinal data sets under the assumption of parameter constraints in linear inequalities.
\end{abstract}




\section{Conteúdo}

Lista de Figuras $\quad$ xi

Lista de Tabelas xvi

1 Introdução 1

1.1 Formulação do problema e definição dos objetivos 1

1.2 Apresentação dos capítulos 2

1.3 Alguns resultados na classe de distribuições simétricas 5

1.3.1 Distribuição Normal 6

$\begin{array}{lll}\text { 1.3.2 Distribuição de Cauchy } & 7\end{array}$

1.3.3 Distribuição $t$-Student 8

1.3.4 Distribuição t-Student Generalizada 9

$\begin{array}{ll}\text { 1.3.5 Distribuição Logística-I } & 10\end{array}$

1.3.6 Distribuição Logística-II 11

$\begin{array}{ll}\text { 1.3.7 Distribuição Logística Generalizada } & 11\end{array}$

1.3.8 Distribuição Exponencial Dupla 12

1.3.9 Distribuição Exponencial Potência 12

1.3.10 Distribuição Potência Estendida 13

$\begin{array}{ll}\text { 1.3.11 Distribuição de Kotz } & 13\end{array}$

1.3.12 Distribuição de Kotz Generalizada 14

$\begin{array}{ll}\text { 1.3.13 Distribuição Normal Contaminada } & 14\end{array}$

2 Modelos de regressão com erros simétricos $\quad 17$

$\begin{array}{lll}2.1 \text { Introdução } & 17\end{array}$ 
CONTEÚDO

2.2 Modelo simétrico de regressão

2.2.1 Informação de Fisher

2.3 Resíduos

2.4 Coelhos europeus na Austrália

3 Modelos simétricos lineares heteroscedásticos 32

3.1 Introdução 32

3.2 Modelos simétricos lineares heteroscedásticos 33

3.2.1 Informação de Fisher 34

3.2.2 Testes de heteroscedasticidade $\quad 35$

$\begin{array}{lll}3.3 \text { Resíduos } & 37\end{array}$

$\begin{array}{ll}3.4 \text { Aplicação } & 39\end{array}$

4 Diagnósticos em modelos simétricos 44

4.1 Influência local no afastamento da verossimilhança 44

4.1.1 Perturbação na escala no modelo simétrico não-linear 46

4.1.2 Perturbação de casos no modelo simétrico linear heterocedástico 46

4.2 Influência local na predição $\quad 47$

4.2.1 Perturbação aditiva na resposta no modelo simétrico linear heteroscedástico

4.2.2 Perturbação na variável explanatória no modelo simétrico linear heteroscedástico

4.3 Ponto de alavanca generalizado no modelo simétrico de regressão 49

4.3.1 Caso linear homoscedástico

4.3.2 Relação entre a medida de influência e a matriz de pontos de alavanca generalizados

4.3.3 Caso linear heteroscedástico $\quad 52$

4.4 Aplicações não-linear (Coelhos) 53

4.5 Aplicação heteroscedástica 59

5 Métodos restritos em modelos simétricos 64 
CONTEÚDO

5.1 Introdução 64

5.2 Inferência com restrições em igualdades e desigualdades lineares $\quad 67$

$\begin{array}{ll}5.2 .1 \text { Igualdades lineares } & 67\end{array}$

$\begin{array}{ll}\text { 5.2.2 Desigualdades lineares } & 69\end{array}$

$\begin{array}{ll}5.3 \text { Testes unilaterais } & 71\end{array}$

$\begin{array}{ll}5.3 .1 \text { Caso } 1 & 71\end{array}$

$\begin{array}{ll}\text { 5.3.2 Caso } 2 & 72\end{array}$

$\begin{array}{ll}5.4 & \text { Assinaturas de TV a cabo }\end{array}$

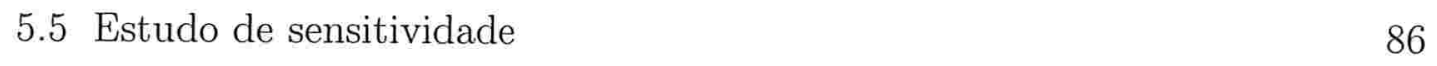

6 Inferência em modelos $t$-multivariados restritos $\quad 89$

6.1 Modelo linear $t$-multivariado 89

$\begin{array}{ll}6.2 g \text { grupos } & 92\end{array}$

6.3 Restrições em igualdades lineares 93

6.4 Restrições em desigualdade lineares 95

$\begin{array}{ll}6.5 \text { Testes unilaterais } & 96\end{array}$

$\begin{array}{ll}\text { 6.6 Ordem simples } & 97\end{array}$

$\begin{array}{ll}6.7 \text { Estudos de simulação } & 98\end{array}$

$\begin{array}{ll}\text { 6.7.1 Dados agrupados } & 99\end{array}$

$\begin{array}{ll}6.7 .2 \text { Presença de regressores } & 110\end{array}$

$\begin{array}{ll}\text { 6.7.3 Dados sobre diabéticos } & 116\end{array}$

$\begin{array}{lr}\text { Conclusões } & 123\end{array}$

A Medidas de curvatura e viés de ordem $n^{-1} \quad 125$

A.1 Multiplicação de "array" 125

$\begin{array}{ll}\text { A.2 Medidas de curvatura } & 126\end{array}$

A.3 Viés de segunda ordem das estimativas de máxima verossimilhança 129

B Probabilidades de Nível 131

$\begin{array}{ll}\text { B.1 Caso de } k=2 \text { restrições } & 131\end{array}$ 
CONTEÚDO

B.2 Caso de $k=3$ restrições 131

B.3 Caso de $k=4$ restrições 132

$\begin{array}{lc}\text { C Coelhos } & 133\end{array}$

$\begin{array}{ll}\text { D Estoque } & 134\end{array}$

$\begin{array}{ll}\text { E TV a cabo } & 135\end{array}$

$\begin{array}{lll}\text { F Pacientes diabéticos } & 136\end{array}$

$\begin{array}{ll}\text { Referências } & 137\end{array}$ 


\section{Lista de Figuras}

1.1 Gráfico da função de densidade da distribuição $t$-Student com $\nu=4$ (esquerda) $\operatorname{com} \nu=6$ (direita).

1.2 Gráfico da função de densidade da distribuição $t$-Student com $\nu=10$ (esquerda) $\operatorname{com} \nu=15$ (direita).

1.3 Gráfico da função de densidade da distribuição exponencial potência com $k=-0,3$ (esquerda) com $k=0,3$ (direita).

1.4 Gráfico da função de densidade da distribuição logística-I (esquerda) e logística-II (direita).

2.1 Gráfico de dispersão do peso das lentes dos olhos contra idade de coelhos europeus.

2.2 Gráfico normal de probabilidades com envelope para $t_{r_{i}}$ (esquerda) e gráfico de resíduos $t_{r_{i}}$ contra os valores ajustados para o modelo normal (direita) ajustado aos dados dos coelhos da Tabela C.1.

2.3 Gráfico normal de probabilidades com envelope para $t_{r_{i}}$ (esquerda) e gráfico de resíduos $t_{r_{i}}$ contra os valores ajustados para o modelo t-Student com 10 g.l. (direita) ajustado aos dados dos coelhos da Tabela C.1.

2.4 Gráfico normal de probabilidades com envelope para $t_{r_{i}}$ (esquerda) e gráfico de resíduos $t_{r_{i}}$ contra os valores ajustados para o modelo logístico-II (direita) ajustado aos dados dos coelhos da Tabela C.1. 
3.1 Gráfico normal de probabilidades com envelope para $r_{t_{i} \text {. (esquerda) }}$ e gráfico de resíduos $r_{t_{i}}$ contra os valores ajustados para o modelo normal (direita), referente ao modelo ajustado aos dados de estoque da Tabela D.1.

3.2 Gráfico normal de probabilidades com envelope para $r_{t_{i}}$ (esquerda) e gráfico de resíduos $r_{t_{i}}$ contra os valores ajustados para o modelo $t$-Student com 4 g.l. (direita), referente ao modelo ajustado aos dados de estoque da Tabela D.1.

3.3 Gráfico normal de probabilidades com envelope para $r_{t_{i}}$ (esquerda) e gráfico de resíduos $r_{t_{i}}$ contra os valores ajustados para o modelo logístico-II (direita), referente ao modelo ajustados aos dados de estoque da Tabela D.1.

4.1 Gráficos de índices de $\mathrm{C}_{i}$ sob o modelo normal (esquerda), $t$-Student com 10 g.l. (direita) e logístico-II (abaixo) ajustados aos dados dos coelhos da Tabela C.1.

4.2 Gráficos de índices de $\mathrm{C}_{i}(\beta)$ sob o modelo normal (esquerda), t-Student com 10 g.l. (direita) e logístico-II (abaixo) ajustados aos dados dos coelhos da Tabela C.1.

4.3 Gráficos de índices de $\mathrm{C}_{i}(\phi)$ sob o modelo normal (esquerda), t-Student com 10 g.l. (direita) e logístico-II (abaixo) ajustados aos dados dos coelhos da Tabela C.1.

4.4 Gráficos de pontos de alavanca generalizados contra idade sob o modelo normal (esquerda), t-Student com 10 g.l. (direita) e logístico-II (abaixo) ajustados aos dados dos coelhos da Tabela C.1.

4.5 Gráfico de $\mathrm{C}_{\max }$ contra $x_{1}$ quando $x_{1}$ é perturbado sob o modelo normal (esquerda), $t$-Student com 4 g.l. (direita) e logístico-II (abaixo) ajustados aos dados de estoque da Tabela D.1. 
4.6 Gráfico de $\mathrm{C}_{\max }$ contra $x_{2}$ quando $x_{2}$ é perturbado sob o modelo normal (esquerda), $t$-Student com 4 g.l. (direita) e logístico-II (abaixo) ajustados aos dados de estoque da Tabela D.1.

4.7 Gráfico de índices dos pontos de alavanca generalizados sob o modelo normal (esquerda), $t$-Student com 4 g.l. (direita) e logístico-II (abaixo) ajustados aos dados de estoque da Tabela D.1.

5.1 Gráfico de $t_{r_{i}}$ contra os valores ajustados para o modelo (5.5) sob erros (a) normal, (b) $t$-Student com 6 g.l., (c) $\operatorname{EP}(0,3)$ e (d) logístico-II.

5.2 Gráfico normal de probabilidades com envelope para o resíduo $t_{r_{i}}$ para o modelo (5.5) sob erro (a) normal, (b) $t$-Student com 6 g.l., (c) $\operatorname{EP}(0,3)$ e (d) logístico-II.

5.3 Gráfico de índices de $\mathrm{C}_{i}$ para as estimativas dos parâmetros do modelo (5.5) sob erro (a) normal, (b) $t$-Student com 6 g.l., (c) $\operatorname{EP}(0,3)$ e (d) logístico-II.

5.4 Gráfico de índices de $\mathrm{C}_{i}(\boldsymbol{\beta})$ para as estimativas dos parâmetros do modelo (5.5) sob erro (a) normal, (b) $t$-Student com 6 g.l., (c) $\operatorname{EP}(0,3)$ e (d) Logístico-II.

5.5 Gráfico de índices de $C_{i}(\phi)$ para as estimativas dos parâmetros do modelo (5.5) sob erro (a) normal, (b) t-Student com 6 g.l., (c) $\operatorname{EP}(0,3)$ e (d) Logístico-II.

5.6 Gráfico de índices de $\mathrm{GL}_{i i}$ para o modelo simétrico perturbado $(a=3)$ sob erros (a) normal (b) $t$-Student com 3 g.l., (c) t-Student com 12 g.l., (d) $\operatorname{EP}(0,3)$, (e) $\operatorname{EP}(0,6)$ e (f) logístico-II.

5.7 Estudo de sensitividade para o $p$-valor da estatística $\xi_{R V}$ sob perturbações na variável explanatória.

6.1 Distribuições acumuladas teórica e empírica da estatística $\xi_{R V}$ para dados agrupados (caso I), $n=20$ e para a estrutura $\mathrm{AR}(1)$. 
6.2 Distribuições acumuladas teórica e empírica da estatística $\xi_{R V}$ para dados agrupados (caso I), $n=20$ e para a estrutura uniforme.

6.3 Distribuições acumuladas teórica e empírica da estatística $\xi_{R V}$ para dados agrupados (caso I), $n=50$ e para a estrutura $\mathrm{AR}(1)$.

6.4 Distribuições acumuladas teórica e empírica da estatística $\xi_{R V}$ para dados agrupados (caso I), $n=50$ e para a estrutura uniforme.

6.5 Distribuições acumuladas teórica e empírica da estatística $\xi_{R V}$ para dados agrupados (caso II), $n_{1}=n_{2}=n_{3}=10$ e para estrutura $\operatorname{AR}(1)$.

6.6 Distribuições acumuladas teórica e empírica da estatística $\xi_{R V}$ para dados agrupados (caso II), $n_{1}=n_{2}=n_{3}=10$ e para estrutura uniforme.

6.7 Distribuições acumuladas teórica e empírica da estatística $\xi_{R V}$ para dados agrupados (caso II), $n_{1}=n_{2}=n_{3}=20$ e para estrutura $\operatorname{AR}(1)$.

6.8 Distribuições acumuladas teórica e empírica da estatística $\xi_{R V}$ para dados agrupados (caso II), $n_{1}=n_{2}=n_{3}=20$ e para estrutura uniforme.

6.9 Distribuições acumuladas teórica e empírica da estatística $\xi_{R V}$ para regressores, $n=20$ e para a estrutura $\mathrm{AR}(1)$.

6.10 Distribuições acumuladas teórica e empírica da estatística $\xi_{R V}$ para regressores, $n=20$ e para a estrutura uniforme.

6.11 Distribuições acumuladas teórica e empírica da estatística $\xi_{R V}$ para regressores, $n=50$ e para a estrutura $\operatorname{AR}(1)$.

6.12 Distribuições acumuladas teórica e empírica da estatística $\xi_{R V}$ para regressores, $n=50$ e para a estrutura uniforme.

6.13 Gráfico de índices do resíduo padronizado $t_{r_{k}}^{*}$ sob o modelo normal. 118

6.14 Gráfico normal de probabilidades com envelope para o resíduo padronizado $t_{r_{k}}^{*}$ sob o modelo normal. 
6.15 Gráfico de índices do resíduo padronizado $t_{r_{k}}^{*}$ sob o modelo $t$-Student.

6.16 Gráfico de probabilidades com envelope para o resíduo padronizado $t_{r_{k}}^{*}$ sob o modelo $t$-Student.

6.17 Comportamentos do $p$-valor para as estatísticas $\xi_{S R}$, $\xi_{R V}$ e $\xi_{W}$ sob o modelo $t$-Student ajustado aos dados de diabéticos.

6.18 Distribuições acumuladas teórica e empírica da estatística $\xi_{R V}$ sob o modelo $t$-Student ajustado aos dados de diabéticos. 


\section{Lista de Tabelas}

2.1 Expressões para $W_{g}(u)$ e $W_{g}^{\prime}(u)$ para algumas distribuições simétricas.

2.2 Valores de $d_{g}, f_{g}$ e $\xi$ para algumas distribuições simétricas.

2.3 Análise descritiva para o resíduo $t_{r_{i}}$ de 1000 observações geradas do modelo ajustado na Seção 2.4 .

2.4 Estimativas de máxima verossimilhança (erro padrão aproximado) para alguns modelos simétricos ajustados aos dados dos coelhos da Tabela C.1.

2.5 Medidas de não-linearidade e viés relativo das estimativas dos parâmetros de locação para alguns modelos simétricos ajustados aos dados dos coelhos da Tabela C.1.

3.1 Análise descritiva para o resíduo $r_{t_{i}}$ de 1000 observações geradas para o modelo ajustado na Seção 3.4.

3.2 Estimativas de máxima verossimilhança (erro padrão aproximado) para alguns modelos simétricos ajustados aos dados de estoque da Tabela D.1.

3.3 Valor das estatísticas dos testes e $p$-valor (entre parênteses) para alguns modelos simétricos ajustados aos dados de estoque da Tabela D.1.

4.1 Mudanças (em \%) nas estimativas dos parâmetros dos modelos ajustados aos dados dos coelhos depois de excluídas as observações $(16,17)$. 
4.2 Mudanças (em \%) nas estimativas dos parâmetros dos modelos ajustados aos dados dos coelhos depois de excluídas as observações $(1,2,3,4,5,16,17)$.

4.3 Mudanças (em \%) nas estimativas dos parâmetros dos modelos ajustados aos dados de estoque depois de excluída a observação 9 .

4.4 Mudanças (em \%) nas estimativas dos parâmetros dos modelos ajustados aos dados de estoque depois de excluída a observação 22.

5.1 Estimativas de máxima verossimilhança irrestritas (erros padrões). 74

5.2 Estimativas de máxima verossimilhança restritas (erros padrões). $\quad 75$

5.3 Valores das estatísticas dos testes e $p$-valor (entre parênteses).

5.4 Valor das estatísticas dos testes e $p$-valor (entre parênteses) excluindo a área 14.

5.5 Valor das estatísticas dos testes e $p$-valor (entre parênteses) excluindo a área 1.

5.6 Mudanças (em \%) nas estimativas dos parâmetros dos modelos irrestritos ajustados aos dados de TV a cabo excluindo as áreas 1 e 14 (erros padrões).

6.1 Estudo de poder dos testes unilaterais e bilaterais para dados agrupados (caso I) e $n=20$.

6.2 Estudo de poder dos testes unilaterais e bilaterais para dados agrupados (caso I) e $n=50$.

6.3 Estudo de poder dos testes unilaterais e bilaterais para dados agrupados (caso I) e $n=100$.

6.4 Diferenças (em valor absoluto) entre a distribuição acumulada teórica e empírica da estatística $\xi_{R V}$ para dados agrupados (caso I) (em \%).

6.5 Estudo de poder dos testes unilaterais e bilaterais para dados agrupados (caso II) e $n_{1}=n_{2}=n_{3}=10$. 
6.6 Estudo de poder dos testes unilaterais e bilaterais para dados agrupados (caso II) e $n_{1}=n_{2}=n_{3}=20$.

6.7 Estudo de poder dos testes unilaterais e bilaterais para dados agrupados (caso II) e $n_{1}=n_{2}=n_{3}=50$.

6.8 Diferenças (em valor absoluto) entre a distribuição acumulada teórica e empírica da estatística $\xi_{R V}$ para dados agrupados (caso II) $(\mathrm{em} \%)$.

6.9 Estudo de poder dos testes unilaterais e bilaterais sob a presença de regressores e $n=20$.

6.10 Estudo de poder dos testes unilaterais e bilaterais sob a presença de regressores e $n=50$.

6.11 Estudo de poder dos testes unilaterais e bilaterais sob a presença de regressores e $n=100$.

6.12 Diferenças (em valor absoluto) entre as distribuições acumuladas teórica e empírica da estatística $\xi_{R V}$ para o caso de regressores (em $\%$ ).

6.13 Estimativas de máxima verossimilhança e valores das estatísticas dos testes sob o modelo normal.

6.14 Estimativas de máxima verossimilhança e valores das estatísticas dos testes sob o modelo $t$-Student $\operatorname{com} \nu=15$ graus de liberdade.

C.1 Pesos das lentes dos olhos de coelhos europeus (y), em miligramas, a idade (x) em dias numa amostra de 71 observações.

D.1 Tempo gasto no serviço (y) em minutos, número de bebidas estocadas $\left(x_{1}\right)$ e distância percorrida $\left(x_{2}\right)$ em pés numa amostra de 25 observações.

E.1 Conjuntos de dados sobre demanda de TV a cabo.

F.1 Efeito de um teste físico em pacientes hospitalares. 


\section{CAPÍTULO 1}

\section{Introdução}

\subsection{Formulação do problema e definição dos objetivos}

A suposição de normalidade sempre foi muito atrativa para os erros de modelos de regressão com resposta contínua e, mesmo quando não era alcançada, procurava-se alguma transformação na resposta no sentido de obter-se pelo menos a simetria. Contudo, com o passar do tempo, verificou-se que as estimativas obtidas para os coeficientes dos modelos normais mostraram-se sensíveis a observações extremas, comumente chamadas de observações aberrantes, incentivando o desenvolvimento de metodologias robustas contra tais observações. Dentre essas metodologias, destacam-se os métodos robustos e modelos robustos. Esses últimos serão discutidos neste trabalho.

$\mathrm{Na}$ linha de modelos robustos, alternativas à suposição de erros normais têm sido propostas na literatura. Uma dessas alternativas é assumir para os erros distribuições com caudas mais pesadas do que a normal, a fim de reduzir a influência de pontos aberrantes. Nessa linha, podemos citar Lange, Little e Taylor (1989) que propõem o modelo $t$-Student com $\nu$ graus de liberdade. Na última década, diversos resultados de natureza teórica e aplicada surgiram como alternativas à modelagem com erros normais como, por exemplo, o uso de distribuições simétricas (ou elípticas). Grande parte desses resultados podem ser encontrados em Fang, Kotz e Ng (1990) e Fang e Anderson (1990).

O objetivo geral deste trabalho é o desenvolvimento da análise inferencial e de validação na classe de modelos lineares e não-lineares com erros simétricos independentes, embora alguns resultados para erros correlacionados serão também apresentados. Podemos então relacionar os seguintes objetivos específicos :

(i) desenvolvimento de processos iterativos na estimação dos parâmetros bem como 
de testes de hipóteses em modelos simétricos de regressão lineares e não-lineares, e modelos simétricos de regressão lineares heteroscedásticos;

(ii) propor resíduos e desenvolver métodos de validação (ou métodos de diagnóstico) em modelos simétricos de regressão lineares e não-lineares;

(iii) desenvolvimento de processos iterativos na estimação dos parâmetros bem como de testes de hipóteses em modelos simétricos de regressão lineares com restrição nos parâmetros;

(iv) desenvolvimento de processos iterativos na estimação dos parâmetros bem como de testes de hipóteses em modelos de regressão $t$-Student multivariados para análise de dados longitudinais.

\subsection{Apresentação dos capítulos}

No capítulo 2 trataremos do problema de estimação dos parâmetros no modelo simétrico de regressão não-linear e testes da razão de verossimilhanças, Wald e escore bem como a caracterização da distribuição nula assintótica e, também, proporemos a definição de um resíduo padronizado. Um dos exemplos motivadores trata-se de uma aplicação analisada em Ratkowsky (1983, Tabela 6.1) sob a suposição de normalidade, cujo interesse principal é relacionar o peso das lentes dos olhos de coelhos europeus (Oryctolagus cuniculus), y (em mg) e a idade do animal, $x$ (em dias), numa amostra de 71 observações. Os dados são encontrados no Apêndice C. Esse animal é largamente distribuído na população selvagem na Austrália. Um aspecto interessante para esses dados que suporta o uso de erros com distribuições de caudas mais pesadas que a normal, é a suspeita de pontos aberrantes na análise por mínimos quadrados. Então, para reanalizar esses dados, propomos o seguinte modelo:

$$
y_{i}=\exp \left(\alpha-\frac{\beta}{x_{i}+\gamma}\right) e^{\epsilon_{i}}, \quad i=1, \ldots, 71,
$$

em que $\epsilon_{i}$ 's são erros mutuamente independentes na classe simétrica de distribuições. 
No capítulo 3 abordaremos os modelos simétricos de regressão em que a heteroscedasticidade é modelada através de um conjunto de variáveis explicativas. Proporemos uma análise de diagnóstico na linha de influência local e um resíduo padronizado. A presença de heteroscedasticidade, frequentemente, aparece em problemas de análise de dados, por exemplo, em dados econométricos. Como ilustração usaremos o conjunto de dados de Montgomery, Peck e Vining (2001, Tabela 3.2), cujo interesse é predizer quanto tempo é requerido pelo motorista da rota para o serviço de manutenção e reposição de latas e garrafas de bebidas em máquinas de vendas automáticas. A atividade de serviço inclue estocagem em máquinas com produtos e sua manutenção. Ajustou-se um modelo linear supondo variância constante cuja variável resposta é o tempo gasto no serviço, y (em minutos), e as covariadas são o número de bebidas estocadas $\left(x_{1}\right)$ e a distância pecorrida pelo motorista $\left(x_{2}\right.$ em pés) numa amostra de 25 observações. Nota-se que as observações 9 e 22 têm grande influência nas estimativas dos parâmetros. Ferrari, Cysneiros e CribariNeto (2004) detectaram a presença de heteroscedasticidade supondo o modelo de regressão linear para o conjunto de dados excluíndo esses pontos. A proposta deste capítulo é de ajustar o modelo heteroscedástico para o conjunto completo de dados com a suposição de erros simétricos. Os dados são encontrados no Apêndice D.

No capítulo 4 desenvolveremos métodos de validação de modelos de regressão simétricos. Seguindo a linha de Cook (1986), proporemos metodologias de influência local, bem como medidas de alavancagem seguindo a linha de Wei, Hu e Fung (1998).

No capítulo 5 trataremos do problema de estimação dos parâmetros restritos em modelos simétricos sob o enfoque da função penalizada quadrática. Desenvolveremos, também, testes da razão de verossimilhanças, Wald e escore para duas situações de interesse, bem como, a caracterização da distribuição nula assintótica. É comum em ensaios clínicos e dados econométricos que os parâmetros estejam sujeitos a algum tipo de restrição. Como exemplo, tem-se um estudo em que sete variáveis são observadas em 40 áreas metropolitanas (veja Apêndice E). O principal interesse é explicar o número (em milhares) de assinantes com TV a cabo 
(y) segundo o número (em milhares) de domicílios na área $\left(x_{1}\right)$, a renda per capita por domicílio com TV a cabo $\left(x_{2}\right)$, a taxa de instalação $\left(x_{3}\right)$, o custo médio mensal de manutenção $\left(x_{4}\right)$, o número de canais a cabo disponíveis na área $\left(x_{5}\right)$ e o número de canais abertos com sinal de boa qualidade na área $\left(x_{6}\right)$. Como $y$ corresponde a dados de contagem usaremos a transformação raiz quadrada a fim de tentar estabilizar a variância de $y$. Então, propomos o modelo

$$
\sqrt{y_{i}}=\beta_{0}+\sum_{j=1}^{6} \beta_{j} x_{j i}+\epsilon_{i}, \quad i=1, \ldots, 40,
$$

em que $\epsilon_{i}$ 's são erros mutuamente independentes na classe de distribuições simétricas. Além disso, é razoável assumir algumas restrições. Por exemplo, é razoável esperar que o número de assinantes decresça à medida que o custo médio mensal de manutenção cresça, embora o contrário possa ocorrer teoricamente. Isso induz à restrição $\beta_{4} \leqslant 0$. Seguindo a mesma idéia para as demais variáveis temos as restrições $\beta_{1} \geqslant 0, \beta_{2} \geqslant 0, \beta_{3} \leqslant 0, \beta_{5} \geqslant 0$ e $\beta_{6} \leqslant 0$.

No capítulo 6 trataremos, também, de modelos de regressão com restrição nos parâmetros com erros $t$-Student multivariados para a análise de dados longitudinais. Um exemplo interessante é o estudo comparativo de indivíduos diabéticos apresentado em Shin, Park e Park (1996). Foram considerados 3 grupos : grupo controle $\left(n_{1}=8\right)$, grupo diabético sem complicações $\left(n_{2}=6\right)$ e grupo diabético com hipertensão $\left(n_{3}=7\right)$. Para cada paciente a resposta foi um teste físico medido em oito intervalos de tempo. Seja $y_{i \ell j}$ a tarefa física observada para o $i$-ésimo paciente do $\ell$-ésimo grupo no tempo $j$. O modelo proposto é o seguinte :

$$
\mathrm{y}_{i \ell}=\mu_{\ell}+\epsilon_{i \ell}
$$

em que $\mu_{\ell}=\mu_{\ell} 1_{m}, \mathrm{y}_{i \ell}=\left(y_{i \ell 1}, \ldots, y_{i \ell m}\right)^{T}$ e $\epsilon_{i \ell}$ segue uma distribuição $t$-Student multivariada de dimensão $m=8 \mathrm{com}$ um vetor de médias zeros e uma matriz escala $\Phi_{i \ell}=\sigma^{2} \mathbf{R}(\rho)$, com $\nu$ graus de liberdade. Como sugerido por Shin, Park e Park (1996) uma estrutura de correlação AR(1) é assumida para $\mathbf{R}(\rho)$. Além disso, é razoável assumir as restrições $\mu_{1} \geqslant \mu_{2} \geqslant \mu_{3}$ para os valores esperados do teste físico. Os dados são encontrados no Apêndice F. 
ALGUNS RESULTADOS NA CLASSE DE DISTRIBUIÇÕES SIMÉTRICAS

O capítulo 7 finaliza esta tese com algumas conclusões e diretrizes para trabalhos futuros.

\subsection{Alguns resultados na classe de distribuições simétricas}

Nesta seção pretendemos caracterizar e apresentar alguns resultados teóricos necessários para o desenvolvimento do texto.

Definição 1.1 Seja a variável aleatória y com suporte em $\mathbb{R}$, com parâmetro de locação $\mu \in \mathbb{R}$ e de escala $\phi>0$ com função de densidade de probabilidade dada por

$$
f(y ; \mu, \phi)=\frac{1}{\sqrt{\phi}} g\left\{\frac{(y-\mu)^{2}}{\phi}\right\}, \quad y \in \mathbb{R},
$$

para alguma função $g(\cdot)$ denominada função geradora de densidades, com $g(u)>0$, para $u>0$ e $\int_{0}^{\infty} u^{-1 / 2} g(u) d u=1$. Essa condição é necessário para que $f(y ; \mu, \phi)$ seja uma função de densidade de probabilidade. Denotamos por $y \sim S(\mu, \phi) e$ denominamos de variável aleatória simétrica.

Como distribuições pertencentes a essa classe podemos citar a normal, $t$-Student, t-Student generalizada, logística tipos I e II, logística generalizada, Kotz, Kotz generalizada, exponencial potência, entre outras.

Algumas propriedades da distribuição normal podem ser estendidas para a classe simétrica de distribuições. Podemos ver que, se $y \sim S(\mu, \phi)$ então a função característica de $y, \varsigma_{y}(t)=\mathrm{E}\left(e^{i t y}\right)$ é dada por $e^{i t \mu} \varphi\left(t^{2} \phi\right), t \in \mathbb{R}$ para alguma função $\varphi$, $\operatorname{com} \varphi(u) \in \mathbb{R}$ para $u>0$. Quando existem, $\mathrm{E}\left(y_{i}\right)=\mu_{i}$ e $\operatorname{Var}\left(y_{i}\right)=\xi \phi$, em que $\xi>0$ é uma constante dada por $\xi=-2 \varphi^{\prime}(0)$, com $\varphi^{\prime}(0)=d \varphi(u) /\left.d u\right|_{u=0}$ e que não depende dos parâmetros $\mu$ e $\phi$ (Fang, Kotz e Ng, 1990, p.43). Kelker (1970) observa que se $u^{-\frac{1}{2}(k+1)} g(u)$ for integrável então o $k$-ésimo momento de $y$ existe.

Temos também que, se $y \sim S(\mu, \phi)$ então $a+b y \sim S\left(a+b \mu, b^{2} \phi\right)$, em que $a, b \in \mathbb{R}$ com $b \neq 0$, isto é, a distribuição de qualquer combinação linear de uma variável aleatória com distribuição simétrica é também simétrica. Como exemplo, se $y \sim S(\mu, \phi)$ então $z=(y-\mu) / \sqrt{\phi} \sim S(0,1)$, com função de densidade $f(z)=$ $f(z ; 0,1)=g\left(z^{2}\right), \dot{z} \in \mathbb{R}$ e chamaremos $z$ de simétrica padrão. 
ALGUNS RESULTADOS NA CLASSE DE DISTRIBUIÇŌES SIMÉTRICAS

Berkane e Bentler (1986) considerando uma distribuição simétrica padrão e que seus momentos existem, mostram que a função característica de $z$ pode ser expandida como

$$
\varsigma_{z}(t)=\sum_{k=0}^{\infty} i^{k} \mu_{k}^{\prime} \frac{t^{k}}{k !}
$$

em que $\mu_{k}^{\prime}=\mathrm{E}\left(y^{k}\right)=i^{-k} \varsigma_{z}^{(k)}(0), \operatorname{com} \varsigma_{z}^{(k)}(0)$ denotando a $k$-ésima derivada de $\varsigma_{z}(t)$ avaliada em $t=0$. Portanto, $\mu_{k}^{\prime}=0$ para $k$ ímpar e para $k=2 m, m=1,2, \ldots$, temos que

$$
\mu_{2 m}^{\prime}=\frac{(2 m) !}{2^{m} m !}\left(\mu_{2}^{\prime}\right)^{m}\{k(m)+1\} \text { e } k(m)=\frac{\varphi^{m}(0)}{\left\{\varphi^{(1)}(0)\right\}^{m}}-1
$$

em que $\varphi^{(r)}(0)$ é a $r$-ésima derivada da função $\varphi$, avaliada em zero. Os coeficientes $k(m), m=1,2, \ldots$ são conhecidos como parâmetros de momentos e generalizam o coeficiente de curtose $\gamma_{2}=3\{k(2)+1\}$ de uma distribuição $S(\mu, \phi)$ (Muirhead, 1982). Cambanis, Huang e Simons (1981) observam que a família de distribuições simétricas coincide com a classe de distribuições elípticas univariadas. Nesta última década surgiram contribuições importantes a partir dos trabalhos de Kelker (1970) para as distribuições elípticas univariadas e multivariadas. Podemos citar algums trabalhos que discutem propriedades dessas distribuições, tais como Berkane e Bentler (1986), Muirhead (1980 e 1982), Rao (1990), Cambanis, Huang e Simons (1981) e Anderson e Fang (1987). Na literatura podemos encontrar excelentes livros, tais como Fang, Kotz e Ng (1990), Fang e Anderson (1990) e Fang e Zhang (1990).

A seguir apresentaremos algumas distribuições simétricas com suporte na reta real para $u=(y-\mu)^{2} / \phi$, em que $y \sim S(\mu, \phi)$.

\subsubsection{Distribuição Normal}

A normal é a distribuição pertencente à classe simétrica mais utilizada devido a todo desenvolvimento teórico e aplicado estabelecido no decorrer dos anos. Alguns resultados devidos a Muirhead (1982), Devlin, Gnanadesikan e Kettenring (1976) caracterizam a distribuição normal, chamada de normal composta, dentro da classe de distribuições simétricas. 
Se $y \sim S(\mu, \phi)$ e a função geradora de densidade $g(\cdot)$ é da forma

$$
g(u)=\frac{1}{\sqrt{2 \pi}} \exp \{-u / 2\}, \quad u>0,
$$

então $y$ tem uma distribuição normal denotada por $y \sim N(\mu, \phi)$, e sua função característica é dada por

$$
\varsigma_{y}(t)=e^{i t \mu} \exp \left\{-t^{2} \phi / 2\right\}, \quad t \in \mathbb{R}
$$

Se $y \sim N(\mu, \phi)$ então $\mathrm{E}(y)=\mu, \operatorname{Var}(y)=\phi$ e os momentos centrais de ordem $r$ são

$$
\mu_{r}=\mathrm{E}\left\{(y-\mu)^{r}\right\}= \begin{cases}0, & r \text { ímpar } \\ \phi^{r / 2} r ! /\left\{2^{r / 2}(r / 2) !\right\}, & r \text { par }\end{cases}
$$

portanto o coeficiente de curtose é $\gamma_{2}=3$.

\subsubsection{Distribuição de Cauchy}

Dizemos que a variável aleatória $y \sim S(\mu, \phi)$ tem distribuição de Cauchy se sua função geradora de densidade $g(\cdot)$ é da forma

$$
g(u)=\frac{1}{\pi}(1+u)^{-1}, \quad u>0 .
$$

Denotamos por $y \sim C(\mu, \phi)$ e sua função característica é dada por

$$
\varsigma_{y}(t)=\exp \{i t \mu-|t| \sqrt{\phi}\}, \quad t \in \mathbb{R}
$$

Em particular, os momentos e os cumulantes para essa distribuição não existem. Sua mediana e moda são iguais a $\mu$, os quartis superior e inferior iguais a $\mu \pm$ $\sqrt{\phi}$. Os pontos de inflexão da função de densidade são $\mu \pm \sqrt{3 \phi}$, e os valores da função de distribuição acumulada nos pontos de inflexão são 0,273 e 0,723 que são próximos aos correspondentes da distribuição normal $(0,159$ e 0,841$)$. A diferença mais importante é que a distribuição de Cauchy tem caudas mais pesadas do que a normal. Um resultado interessante é que para $a_{j} \neq 0, \sum_{j=1}^{n} a_{j} y_{j}$ e $y_{j} \sim$ $C\left(\mu_{j}, \phi_{j}\right)$ independentes temos uma distribuição de Cauchy com parâmetros de locação $\mu=\sum_{i=1}^{n} a_{j} \mu_{j}$ e escala, $\phi=\sum_{i=1}^{n} a_{j}^{2} \phi_{j}$. Em particular, se $y_{j}$ são i.i.d. então 

$\bar{y}=n^{-1} \sum_{i=1}^{n} y_{j} \sim C(\mu, \phi)$. A distribuição de Cauchy padronizada reduz-se $(\mu=0$ e $\phi=1$ ) à distribuição central $t$-Student com um grau de liberdade. Temos ainda a relação $y=\mu+\phi N_{1} / N_{2}$ em que $N_{i} \sim N(0,1)$ para $i=1,2$ independentes. Com essa relação é possível definir um gerador de números aleatórios para a distribuição de Cauchy.

\subsubsection{Distribuição t-Student}

A variável aleatória $y$ tem distribuição $t$-Student com $\nu$ graus de liberdade se $y \sim S(\mu, \phi)$ e a sua função geradora de densidades for da forma

$$
g(u)=\frac{\nu^{\nu / 2}}{\mathrm{~B}(1 / 2, \nu / 2)}(\nu+u)^{-\frac{\nu+1}{2}}, \quad \nu>0, u>0,
$$

em que $\mathrm{B}(\cdot, \cdot)$ é a função Beta e denotamos $y \sim t(\mu, \phi, \nu)$. Logo, a função de densidade de $y$ é obtida de (1.1) aplicando a função $g(\cdot)$ acima. Podemos encontrar a sua função característica definida em Fang, Kotz e Ng (1990, p.87). Relacionando algumas propriedades temos que se $y$ é definido por $y=v^{1 / 2} z$, em que $v \sim G I(\nu / 2, \nu / 2)$ (gama inversa), $\nu>0$ e $z \sim N(0,1)$ independentes, então, $y \sim t(0,1, \nu)$.

Se $t(0,1, \nu)$ temos o seguinte :

(i) Para $\nu>r$, seus momentos de ordem $r$ existem e são dados por

$$
\mathrm{E}\left(y^{r}\right)= \begin{cases}0, & r \text { ímpar } \\ \nu^{r / 2} \Gamma\left(\frac{r+1}{2}\right) \Gamma\left(\frac{\nu-r}{2}\right) /\left\{\Gamma\left(\frac{1}{2}\right) \Gamma\left(\frac{\nu}{2}\right)\right\}, & r \text { par }\end{cases}
$$

em que $\Gamma(\cdot)$ denota a função Gama. Logo, $\mathrm{E}(y)=0$ para $\nu>1$ e $\operatorname{Var}(y)=$ $\nu /(\nu-2)$ para $\nu>2$. Se $r \geqslant \nu$ e $r$ par temos que o momento de ordem $r$ é infinito;

(ii) o desvio médio é dado por

$$
\mathrm{E}(|y|)=\frac{\nu^{1 / 2} \Gamma\left(\frac{\nu-1}{2}\right)}{\Gamma(1 / 2) \Gamma(\nu / 2)}
$$

(iii) o coeficiente de curtose é dado por $\gamma_{2}=3+6 /(\nu-4)$, para $\nu>4$. Observe que este coeficiente é maior do que o coeficiente da distribuição normal. 
(iv) $y^{2} \sim F_{(1, \nu)}$ em que $F_{(1, \nu)}$ denota a distribuição $F$-Snedecor com 1 e $\nu$ graus de liberdade;

(v) se $w=(\nu+1) /\left(\nu+y^{2}\right)$ então

$$
\mathrm{E}\left(y^{2 k} w^{\ell}\right)=\frac{\left(-\frac{\nu+1}{2}\right)^{\ell}}{\nu^{\ell-k}} \frac{\mathrm{B}[(2 k+1) / 2,\{\nu+2(\ell-k)\} / 2]}{\mathrm{B}(1 / 2, \nu / 2)},
$$

para $\ell=0,1,2$ e $k=1,2, \ldots$;

(vi) a função densidade de $y$ tem pontos de inflexão em $\pm\{\nu /(\nu+2)\}^{1 / 2}$;

(vii) a variável aleatória $u=\left(1+\nu / y^{2}\right)^{-1}$ tem distribuição beta com parâmetros $a=1 / 2$ e $b=\nu / 2$ (Manoukin , 1985, p.41);

(viii) $y \mid v=\nu \sim N(0, \nu)$;

(ix) $v \mid y=y \sim G I\left\{(\nu+1) / 2,\left(\nu+y^{2}\right) / 2\right\}$.

Baseados nessas propriedades podemos ver que a distribuição $t$-Student de parâmetros $(\mu, \phi, \nu)$ tende a um distribuição normal com média $\mu$ e variância $\phi$ quando $\nu \rightarrow \infty$.

Quando $\nu=1$ temos a distribuição de Cauchy com parâmetros $\mu$ e $\phi$.

\subsubsection{Distribuição t-Student Generalizada}

Uma variável aleatória $y \sim S(\mu, \phi)$ com a função geradora de densidades definida por

$$
g(u)=\frac{s^{r / 2}}{\mathrm{~B}(1 / 2, r / 2)}(s+u)^{-\frac{r+1}{2}}, \quad s, r>0, u>0,
$$

é dita $t$-Student generalizada com parâmetros $(\mu, \phi, s, r)$ (Dickey, 1967). Como membro dessa família de distribuições temos a $t$-Student $(s=r=\nu)$ e Cauchy $(s=r=1)$. Quando $\sqrt{s}=c$ e $(r+1) / 2=m$, com $m>1 / 2$ temos a distribuição Pearson VII (Fang, Kotz e Ng, 1990).

Suponha $y \mid v=\nu \sim N(\mu, \nu \phi)$, em que $v \sim G I(r / 2, s / 2)$, independentes com $s, r>0$ podendo não ser inteiro. Podemos relacionar algumas propriedades :

(i) $y \sim t G(\mu, \phi, s, r)$;

(ii) $\mathrm{E}(y)=\mu$ para $r>1, \operatorname{Var}(y)=\{s /(r-2)\} \phi$ para $r>2$ e o coeficiente de curtose $\gamma_{2}=3+6 /(r-4)$ para $r>4$. Vale salientar que o coeficiente de curtose não depende do parâmetro $s$ e é maior do que o coeficiente de curtose da normal; 
ALGUNS RESULTADOS NA CLASSE DE DISTRIBUIÇŌES SIMÉTRICAS

(iii) $v \mid y \sim G I\left\{(r+1) / 2,\left(s+z^{2}\right) / 2\right\}$, em que $z^{2}=(y-\mu)^{2} / \phi$;

(iv) $u^{2}=r z^{2} / s \sim F_{(1, r)}$;

$(v)$ se $w=(r+1) /\left(s+z^{2}\right)$ então

$$
\mathrm{E}\left(z^{2 k} w^{\ell}\right)=\frac{\left(-\frac{r+1}{2}\right)^{\ell}}{s^{\ell-k}} \frac{\mathrm{B}[(2 k+1) / 2,\{r+2(\ell-k)\} / 2]}{\mathrm{B}(1 / 2, r / 2)}
$$

para $\ell=0,1,2$ e $k=1,2, \ldots$;

(vi) os parâmetros $s$ e $r$ tem uma relação com o parâmetro de curtose e o segundo momento central (Johnson e Kotz, 1970, p.116) dados por

$$
r=\frac{2\left(2 \gamma_{2}-3\right)}{\gamma_{2}-3} \quad \text { e } \quad s=\frac{2 \mu_{2} \gamma_{2}}{\gamma_{2}-3}
$$

(vii) o $\ell$-ésimo momente existe se e somente se $r>\ell$;

(viii) para a variável aleatória $y=v^{-1 / 2} z, z$ e $v$ variáveis aleatórias independentes, em que $z \sim N(0,1)$ e $v \sim G I(r / 2, s / 2)$ então $y \sim t G(0,1, s, r)$.

\subsubsection{Distribuição Logística-I}

Dizemos que a variável aleatória $y \sim S(\mu, \phi)$ tem distribuição logística-I (Fang, Kotz e $\mathrm{Ng}, 1990)$ se sua função geradora de densidades $g(\cdot)$ é da forma

$$
g(u)=c \frac{e^{-u}}{\left(1+e^{-u}\right)^{2}}, \quad u>0
$$

em que $c$ é a constante normalizadora obtida da relação $\int_{0}^{\infty} u^{-1 / 2} g(u)=1$, logo $c \approx 1,484300029$ e é denotada por $y \sim L I(\mu, \phi)$. Temos que $\mathrm{E}(y)=\mu, \operatorname{Var}(y) \approx$ $0,79569 \phi$ e $\gamma_{2} \approx 2,385165$. Observe que o coeficiente de curtose da distribuição logística-I é menor do que o coeficiente de curtose da distribuição normal.

Se $v=\left(e^{-z^{2}}-1\right) /\left(1+e^{-z^{2}}\right)$, com $z^{2}=(y-\mu)^{2} / \phi$, então $\mathrm{E}\left(z^{2 r} v^{\ell}\right)=\frac{c}{2}(-1)^{\ell} \int_{0}^{1}\{\log (1+s)-\log (1-s)\}^{r-1 / 2} s^{\ell} d s, \quad \ell=0,1,2, \ldots$ e $r=1,2, \ldots$ 


\subsubsection{Distribuição Logística-II}

Dizemos que a variável aleatória $y \sim S(\mu, \phi)$ tem distribuição logística-II se sua função geradora de densidades $g(\cdot)$ é da forma

$$
g(u)=\frac{e^{-u^{1 / 2}}}{\left(1+e^{-u^{1 / 2}}\right)^{2}}, \quad u>0,
$$

denotada por $y \sim L I I(\mu, \phi)$. A função característica é dada por $\varsigma_{y}(t)=\frac{2\left(\mathrm{e}^{i t \mu} \pi \phi^{1 / 2} t\right)}{\left(\mathrm{e}^{\pi \phi^{1 / 2} t}-\mathrm{e}^{-\pi \phi^{1 / 2}} t\right)}$, $t \in \mathbb{R}$. Temos que $\mathrm{E}(y)=\mu, \operatorname{Var}(y)=\pi^{2} \phi / 3$ e $\gamma_{2}=4,2$. E ainda, tem-se que a mediana e moda são iguais à média. Uma relação bastante útil para gerar amostras aleatórias é dada por Hastings e Peacock (1975). Seja $u \sim \mathrm{U}(0,1)$ e $y=\mu+\sqrt{\phi} \log \{u /(1-u)\}$ então $y \sim L I I(\mu, \phi)$. A função de distribuição logística-II é comumente usada para representar curvas de crescimento em economia e demografia (Johnson e Kotz, 1970).

\subsubsection{Distribuição Logística Generalizada}

Uma variável aleatória $y \sim S(\mu, \phi)$ tem distribuição logística generalizada se a sua função geradora de densidades $g(\cdot)$ é da forma

$$
g(u)=\frac{\alpha}{\mathrm{B}(m, m)}\left\{\frac{\mathrm{e}^{-\alpha \sqrt{u}}}{\left(1+\mathrm{e}^{-\alpha \sqrt{u}}\right)^{2}}\right\}^{m}, \quad m>0, u>0
$$

em que $\alpha=\alpha(m)$ com $\alpha(\cdot)$ definida em $\mathbb{R}^{+}$e $\alpha(m)>0$, para $m>0$, e é denotada por $y \sim L G(\mu, \phi, m)$. Essa distribuição pertence à família de distribuições de Perks (veja Johnson e Kotz, 1970). Se $\alpha(m)=1, \forall m>0$ e $m=1$ temos a distribuição logística-II. Gumbel (1944) utiliza a distribuição logística generalizada com uma função particular $\alpha(\cdot)$ para a distribuição da $m$-ésima amplitude (média entre o maior e o menor valor de uma amostra aleatória de tamanho $n$ ) para uma classe de distribuições simétricas. Temos que $\mathrm{E}(y)=\mu, \operatorname{Var}(y)=2 \psi^{\prime}(m) \phi / \alpha(m)$ e $\gamma_{2}=3+\frac{\psi^{\prime \prime \prime}(m)}{2 \psi^{\prime}(m)^{2}}$, em que $\psi^{\prime}(\cdot)$ e $\psi^{\prime \prime \prime}(\cdot)$ são a primeira e a terceira derivadas da função digama, respectivamente e $\forall m>0$ temos que $\gamma_{2}>0$. Quando $m \rightarrow \infty$ temos que $\gamma_{2} \rightarrow 3$, ou melhor, o coeficiente de curtose da logística generalizada converge para o coeficiente de curtose da normal. 


\subsubsection{Distribuição Exponencial Dupla}

Uma variável aleatória $y \sim S(\mu, \phi)$ tem distribuição exponencial dupla (Laplace) se a sua função geradora de densidades $g(\cdot)$ é da forma

$$
g(u)=\frac{1}{2} \exp \{-\sqrt{u}\}, \quad u>0,
$$

e denotamos por $y \sim E D(\mu, \phi)$. A função característica é dada por $\varsigma_{y}(t)=$ $\frac{e^{i t \mu}}{1+t^{2} \phi}, t \in \mathbb{R}$. Se $z \sim E D(0,1)$ temos os momentos $\mu_{r}^{\prime}$ dados por

$$
\mu_{r}^{\prime}=\mathrm{E}\left(z^{r}\right)= \begin{cases}0, & r \text { ímpar } \\ r !, & r \text { par. }\end{cases}
$$

Portanto, $\mathrm{E}(y)=\mu, \operatorname{Var}(y)=2 \phi$, a mediana e a moda são iguais a $\mu$ e ainda o coeficiente de curtose $\gamma_{2}=6$. Os quartis superior e inferior são $\mu \pm 0,534 \sqrt{\phi}$.

\subsubsection{Distribuição Exponencial Potência}

Uma variável aleatória $y \sim S(\mu, \phi)$ tem distribuição exponencial potência (Box e Tiao, 1973, Cap. 3) se a sua função geradora de densidades $g(\cdot)$ é da forma

$$
g(u)=C(k) \exp \left\{-\frac{1}{2} u^{1 /(1+k)}\right\}, \quad-1<k \leqslant 1, u>0,
$$

em que $C(k)^{-1}=\Gamma\left(1+\frac{1+k}{2}\right) 2^{1+(1+k) / 2}$ e denotamos por $y \sim E P(\mu, \phi, k)$.

Temos ainda que

$$
\mathrm{E}(y)=\mu, \operatorname{Var}(y)=2^{(1+k)}\left[\frac{\Gamma\left\{\frac{3(1+k)}{2}\right\}}{\Gamma\left(\frac{1+k}{2}\right)}\right] \phi \text { e } \gamma_{2}=\frac{\Gamma\left\{\frac{5}{2}(1+k)\right\} \Gamma\left(\frac{1+k}{2}\right)}{\Gamma^{2}\left\{\frac{3}{2}(1+k)\right\}}
$$

Observe que para $k>0$, temos que $\gamma_{2}>3$, ou seja, a distribuição é leptocúrtica e para $k<0$, temos $\gamma_{2}<3$, ou seja, a distribuição é platicúrtica. Podemos ver o parâmetro $k$ como uma medida de curtose, ou mesmo, uma medida de não normalidade pois quando $k=0$ temos a distribuição normal. Em particular, quando $k=1$ temos a distribuição exponencial dupla. Se $k$ tende a -1 , a distribuição tende a uma distribuição uniforme no intervalo $(\mu-\sqrt{3 \phi}, \mu+\sqrt{3 \phi})$.

Se $y=(2 w)^{1 / r} v$ em que $v \sim \mathrm{U}(-1,1), w \sim G(1+1 / r, 1)$ e $r=2 /(1+k)$ independentes (veja Devroye, 1986, pp.174-175), então $y \sim E P(0,1, k)$. Essa relação é suficiente para gerar amostras de uma distribuição $\operatorname{EP}(0,1, k)$. 


\subsubsection{Distribuição Potência Estendida}

Uma variável aleatória $y \sim S(\mu, \phi)$ tem distribuição potência estendida (Albert, Delampady e Polasek, 1991) se a sua função geradora de densidades $g(\cdot)$ é da forma

$$
g(u)=C(c, \lambda) \exp \left[-\frac{1}{2} c \rho_{\lambda}\{1+u /(c-1)\}\right],
$$

denotamos por $y \sim P E(\mu, \phi, \lambda)$ em que $C(c, \lambda)$ é uma constante normalizadora, $c>1, \lambda \geqslant 0, u>0 \mathrm{e}$

$$
\rho_{\lambda}(v)= \begin{cases}\frac{v^{\lambda}-1}{\lambda}, & \text { se } \lambda>0 \\ \lim _{\lambda \rightarrow 0} \frac{v^{\lambda}-1}{\lambda}, & \text { se } \lambda=0 .\end{cases}
$$

Podemos citar alguns casos particulares, quando $\lambda=1$ temos a distribuição $N(\mu, \phi\{c-1\} / c)$, se $\lambda=0$ temos a distribuição $t$-Student $(\mu, \phi, c-1)$ e quando $\lambda=1 / 2$ temos a distribuição exponencial dupla. Se $\lambda>0$, os momentos $\mathrm{E}\left(y^{k}\right)$ existem para $k>0$.

\subsubsection{Distribuição de Kotz}

Dizemos que uma variável aleatória $y \sim S(\mu, \phi)$ tem distribuição de Kotz (Kotz, 1975) se a sua função geradora de densidades $g(\cdot)$ é da forma

$$
g(u)=\frac{r^{(2 N-1) / 2}}{\Gamma\left(\frac{2 N-1}{2}\right)} u^{N-1} \mathrm{e}^{-r u}, \quad r>0, N \geqslant 1, u>0,
$$

e denotamos por $y \sim K(\mu, \phi, N, r)$. Quando $N=1$ temos a distribuição normal com média $\mu$ e variância $\phi /(2 r)$. Ainda se $N>1$, a distribuição é bimodal com modas em $y=\mu \pm \sqrt{(N-1) /(r \phi)}$. Temos que $\mathrm{E}(y)=\mu, \operatorname{Var}(y)=\{(2 N-$ 1) $/(2 r)\} \phi$, o coeficiente de curtose $\gamma_{2}=(2 N+1) /(2 N-1)$ e os momentos centrais de ordem $2 m$ dados por

$$
\mu_{2 m}=\mathrm{E}\left\{(y-\mu)^{2 m}\right\}=\frac{\Gamma\{(2 N+2 m-1) / 2\}}{r^{m} \Gamma\{(2 N-1) / 2\}} \phi^{m}, m>0 .
$$

Se $z^{2}=(y-\mu)^{2} / \phi$ então $z^{2} \sim G(\{2 N-1\} / 2, r)$. Em particular, se $N=1$ e $r=1 / 2$ então temos que $z^{2} \sim \chi_{1}^{2}$. 


\subsubsection{Distribuição de Kotz Generalizada}

Seja $y \sim S(\mu, \phi)$ com a função geradora de densidades $g(\cdot)$ dada por

$$
g(u)=\frac{s r^{(2 N-1) / 2 s}}{\Gamma\left(\frac{2 N-1}{2 s}\right)} u^{N-1} \mathrm{e}^{-r u^{s}}, \quad r, s>0, N \geqslant 1, u>0 .
$$

Então $y$ tem distribuição de Kotz generalizada e denotamos por $y \sim K G(\mu, \phi, N, r, s)$. Quando $s=1$ a distribuição reduz a $K(\mu, \phi, N, r)$ e, quando $N=1, s=1$ e $r=1 / 2$ temos a distribuição normal $N(\mu, \phi)$. Ainda, se $N=1, r=1 / 2$ e $s=1 /(1+k)$ temos a distribuição exponencial potência.

Temos que

$\mathrm{E}(y)=\mu, \operatorname{Var}(y)=\frac{\Gamma\{(2 N-1) / 2 s\}}{r^{1 / s} \Gamma\{(2 N-1) / 2 s\}} \phi$ e $\gamma_{2}=\frac{\Gamma\{(2 N-1) / 2 s\} \Gamma\{(2 N+3) / 2 s\}}{\Gamma^{2}\{(2 N+1) / 2 s\}}$ e os momentos centrais de ordem $2 m$ são dados por

$$
\mu_{2 m}=\mathrm{E}\left\{(y-\mu)^{2 m}\right\}=\frac{\Gamma\{(2 N+2 m-1) / 2 s\}}{r^{m / s} \Gamma\{(2 N-1) / 2 s\}} \phi^{m}, \quad m>0 .
$$

\subsubsection{Distribuição Normal Contaminada}

Considere uma variável aleatória $y \sim S(\mu, \phi)$ com a função geradora de densidades $g(\cdot)$ dada por

$$
g(u)=(1-\epsilon) \frac{1}{\sqrt{2 \pi}} \exp \{-u / 2\}+\epsilon \frac{1}{\sqrt{2 \pi \sigma}} \exp \left\{-u /\left(2 \sigma^{2}\right)\right\},
$$

em que $u>0, \sigma>0$ e $0 \leqslant \epsilon \leqslant 1$ e denotaremos $y \sim N C\left(\mu, \phi, \epsilon, \sigma^{2}\right)$. Temos que $\mathrm{E}(y)=\mu$ e $\operatorname{Var}(y)=\left\{1+\epsilon\left(\sigma^{2}-1\right)\right\} \phi$. O coeficiente de curtose fica dado por (Berkane e Bentler, 1986)

$$
\gamma_{2}=\frac{3\left\{1+\epsilon\left(\sigma^{4}-1\right)\right\}}{\left\{1+\epsilon\left(\sigma^{2}-1\right)\right\}^{2}} .
$$

Little (1988) incorpora parâmetros adicionais para ajustar a curtose utilizando esta distribuição.

Como ilustração, temos os gráficos da função de densidade de várias distribuições simétricas (linha cheia) comparando com a função de densidade da distribuição normal (linha pontilhada). Para todas as distribuições aqui consideradas, o parâmetro de locação e escala são fixados em $\mu=0$ e $\phi=1$, respectivamente. 
Figura 1.1 Gráfico da função de densidade da distribuição t-Student com $\nu=4$ (esquerda) $\operatorname{com} \nu=6$ (direita).
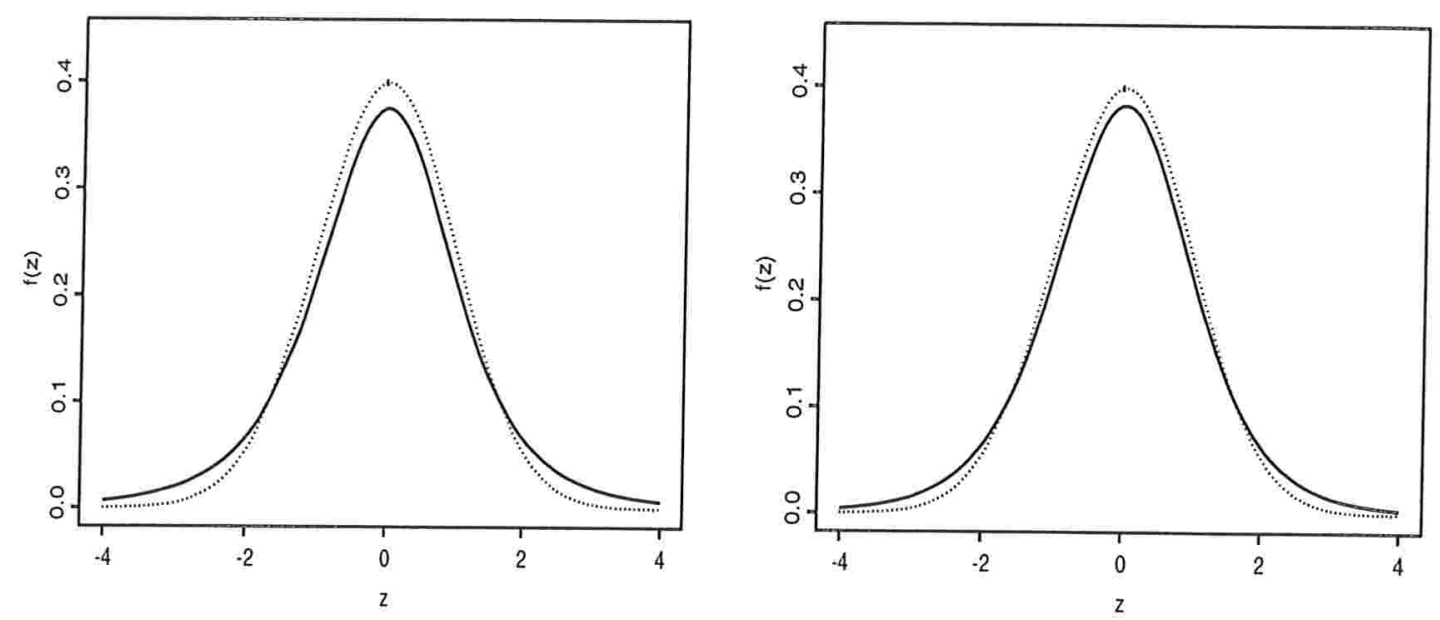

Figura 1.2 Gráfico da função de densidade da distribuição t-Student com $\nu=10$ (esquerda) $\operatorname{com} \nu=15$ (direita).
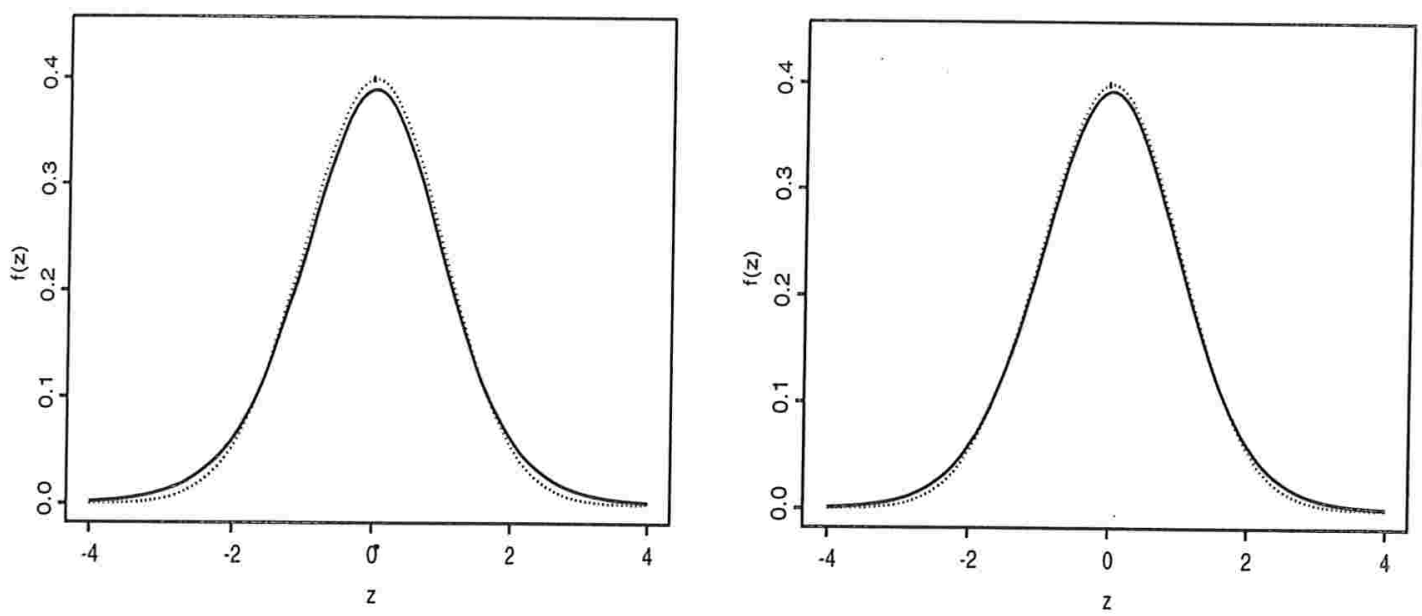
Figura 1.3 Gráfico da função de densidade da distribuição exponencial potência com $k=-0,3$ (esquerda) com $k=0,3$ (direita).
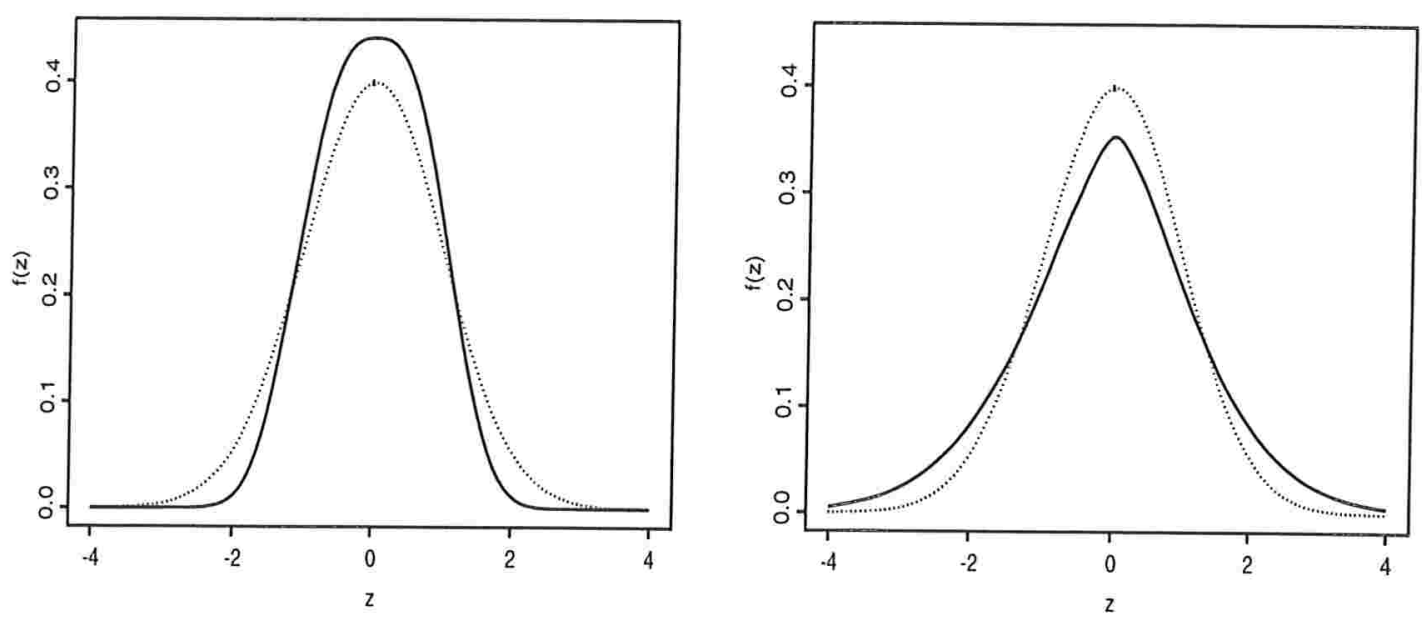

Figura 1.4 Gráfico da função de densidade da distribuição logística-I (esquerda) e logística-II (direita).
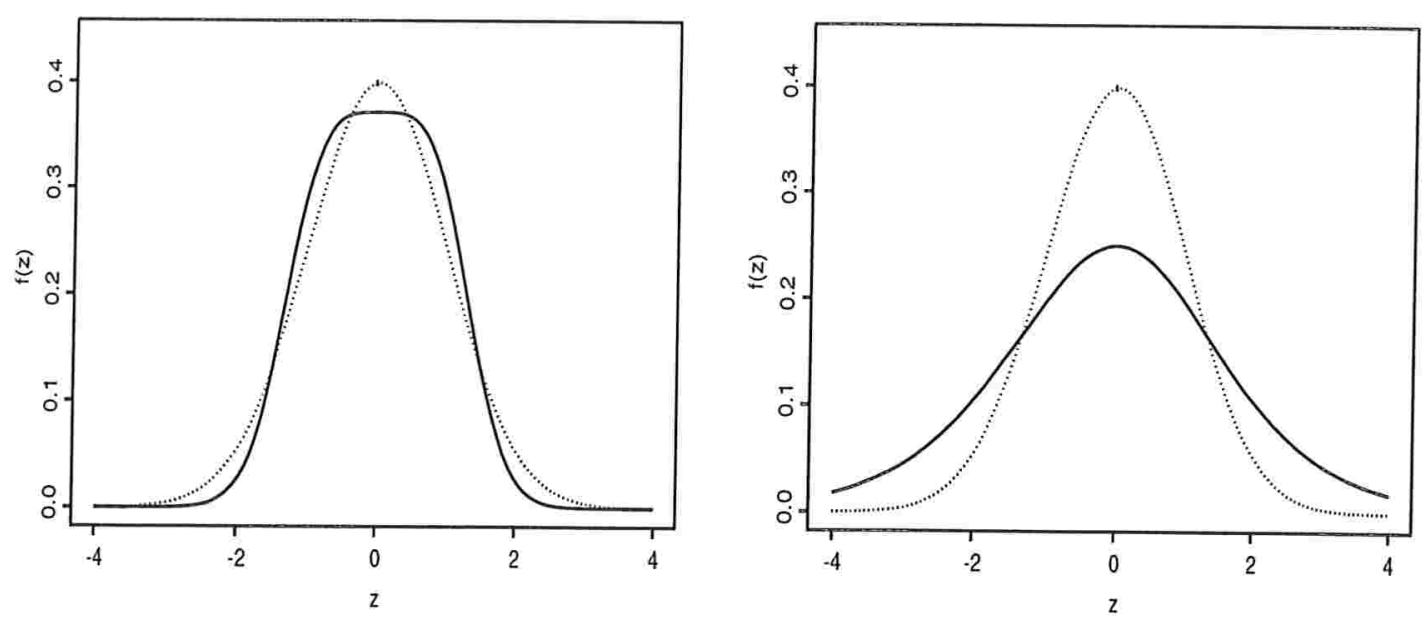


\section{CAPÍTULO 2}

\section{Modelos de regressão com erros simétricos}

\subsection{Introdução}

A classe de distribuições simétricas tem recebido uma crescente atenção na literatura estatística nos últimos anos (veja por exemplo, Fang, Kotz e Ng, 1990; Fang e Zhang, 1990; Fang e Anderson, 1990 e Gupta e Varga, 1993). Uma revisão de diferentes áreas em que as distribuições simétricas são aplicadas é descrita em Chmielewski (1981). Em muitas situações da modelagem estatística há necessidade da procura de modelos menos sensíveis a observações aberrantes. É bem conhecido que os estimadores obtidos pelo método de mínimos quadrados são altamente sensíveis a observações aberrantes. Como alternativa robusta, Lange, Little e Taylor (1989) propõem o modelo baseado na suposicão de erros $t$-Student enquanto Little (1988) e Yamaguchi (1990) utilizam a distribuição normal contaminada. Em ambos os modelos incorporam-se parâmetros adicionais, os quais permitem ajustar a curtose da distribuição aos dados. No caso da $t$-Student, os graus de liberdade são usados para controlar a curtose. Taylor (1992) propõe o ajuste de um modelo de regressão linear supondo erros distribuídos como exponencial potência com um parâmetro extra de forma. Albert, Delampady e Polasek (1991) estendem resultados para a família potência estendida estudando propriedades robustas no enfoque de estimação dos parâmetros do modelo de regressão. Arellano-Valle (1994) apresenta vários resultados para a $t$-Student com aplicações em modelos com erros nas variáveis. Ferrari e Arellano-Valle (1996) desenvolvem correções de Bartlett para teste de hipóteses em modelos de regressão linear com erros $t$-Student e Uribe-Opazo (1997) e Ferrari e Uribe-Opazo (2001) estendem esses resultados para modelos de regressão linear com erros simétricos. Uribe-Opazo, Ferrari e Cordeiro (2003) desenvolvem correções tipo-Bartlett para modelos de regressão linear 
MODELO SIMÉTRICO DE REGRESSÃO

com erros simétricos e Cordeiro (2004) desenvolveu correções de Bartlett para os modelos de regressão não-lineares simétricos .

\subsection{Modelo simétrico de regressão}

Para definir a classe de modelos de regressão com erros simétricos suponha que $\epsilon_{1}, \ldots, \epsilon_{n}$ são variáveis aleatórias independentes com função de densidade definida como

$$
f_{\epsilon_{i}}(\epsilon)=\frac{1}{\sqrt{\phi}} g\left\{\epsilon^{2} / \phi\right\}
$$

$\epsilon \in \mathbb{R}$ and $g(\cdot)$ definida como na Seção 1.3. O modelo simétrico não-linear é definido aqui por

$$
y_{i}=\mu_{i}\left(\beta ; \mathbf{x}_{i}\right)+\epsilon_{i}
$$

em que $\mu_{i}=\mu_{i}\left(\boldsymbol{\beta} ; \mathbf{x}_{i}\right)$ é uma função não-linear contínua e diferenciável de $\boldsymbol{\beta}=$ $\left(\beta_{1}, \ldots, \beta_{p}\right)^{T}$ tal que a matriz de derivadas $\mathbf{D}_{\beta}=\frac{\partial \boldsymbol{\mu}}{\partial \boldsymbol{\beta}}$ tenha posto $p(p<n)$ para todo $\boldsymbol{\beta}$ com $\boldsymbol{\mu}=\left(\mu_{1}, \ldots, \mu_{n}\right)^{T}, \mathbf{y}=\left(y_{1}, \ldots, y_{n}\right)^{T}$ é o vetor de respostas observadas, $\mathrm{x}_{i}=\left(x_{i 1}, \ldots, x_{i n}\right)^{T}$ contém valores de $p$ variáveis explanatórias e $\epsilon_{i} \sim S(0, \phi)$. No caso linear tem-se que $\boldsymbol{\mu}=\mathbf{X} \boldsymbol{\beta} \operatorname{com} \mathbf{X}=\left(\mathbf{x}_{1}^{T}, \ldots, \mathbf{x}_{n}^{T}\right)^{T}$. A densidade de $y_{i}$ é dada por

$$
f_{y_{i}}\left(y_{i}\right)=\frac{1}{\sqrt{\phi}} g\left(u_{i}\right)
$$

em que $u_{i}=\left(y_{i}-\mu_{i}\right)^{2} / \phi$ e $y_{i} \sim S\left(\mu_{i}, \phi\right)$. Quando existem, $\mathrm{E}\left(y_{i}\right)=\mu_{i}$ e $\operatorname{Var}\left(y_{i}\right)=$ $\xi \phi$. O modelo definido por (2.2) e (2.3) é dito modelo simétrico de regressão nãolinear. O logaritmo da função de verossimilhança de $\theta=\left(\beta^{T}, \phi\right)^{T}$ é dado por

$$
\mathrm{L}(\boldsymbol{\theta})=-\frac{n}{2} \log \phi+\sum_{i=1}^{n} \log \left\{g\left(u_{i}\right)\right\} .
$$

A função $L(\theta)$ é assumida ser regular (Cox e Hinkley, 1974, Cap. 9) com respeito a $\boldsymbol{\beta}$ e $\phi$. Condições regulares são encontradas, também, em Serfling (1980, p. 144). Para obter a função escore e as matrizes de informação de Fisher precisamos derivar $\mathrm{L}(\boldsymbol{\theta})$ com respeito aos parâmetros desconhecidos e então calcular alguns momentos dessas derivadas. Supomos aqui que tais derivadas existem. Contudo, 
algumas distribuições simétricas não satisfazem as condições de regularidade, por exemplo, exponencial dupla. Esses casos não serão considerados.

As funções escore para $\beta$ e $\phi$ tomam, respectivamente, as formas

$$
\mathrm{U}_{\beta}(\theta)=\frac{1}{\phi} \mathrm{D}_{\beta}^{T} \mathrm{D}(\mathrm{v})(\mathrm{y}-\mu)
$$

e

$$
\mathrm{U}_{\phi}(\theta)=-\frac{1}{\phi} \sum_{i=1}^{n}\left\{\frac{1}{2}+W_{g}\left(u_{i}\right) u_{i}\right\}=-\frac{n}{2 \phi}+\frac{1}{2 \phi^{2}}(\mathrm{y}-\mu)^{T} \mathrm{D}(\mathrm{v})(\mathrm{y}-\mu),
$$

em que $\mathbf{D}(\mathbf{v})=\operatorname{diag}\left\{v_{1}, \ldots, v_{n}\right\}$ com $v_{i}=-2 W_{g}\left(u_{i}\right)$. Expressões para $W_{g}(u)$ e $W_{g}^{\prime}(u)$ para algumas distribuições simétricas são dadas na Tabela 2.1. Algoritmos de estimação são discutidos em Smyth (1996). Um processo iterativo para obter as estimativas de máxima verossimilhança de $\boldsymbol{\beta}$ e $\phi$ pode ser desenvolvido usando, por exemplo, o método scoring de Fisher. O processo iterativo conjunto é dado por

$$
\boldsymbol{\beta}^{(m+1)}=\boldsymbol{\beta}^{(m)}+\left(4 d_{g}\right)^{-1}\left\{\mathbf{D}_{\beta}^{T(m)} \mathbf{D}_{\beta}^{(m)}\right\}^{-1} \mathbf{D}_{\beta}^{(m) T} \mathbf{D}\left(\mathbf{v}^{(m)}\right)\left\{\mathbf{y}-\boldsymbol{\mu}\left(\boldsymbol{\beta}^{(m)}\right)\right\}
$$

e

$$
\phi^{(m+1)}=\frac{1}{n} Q_{V}\left(\boldsymbol{\beta}^{(m+1)}\right) \quad(m=0,1,2, \ldots),
$$

em que $Q_{V}(\boldsymbol{\beta})=\{\mathrm{y}-\boldsymbol{\mu}(\boldsymbol{\beta})\}^{T} \mathbf{D}(\mathrm{v})\{\mathrm{y}-\boldsymbol{\mu}(\boldsymbol{\beta})\}, d_{g}=\mathrm{E}\left\{W_{g}^{2}\left(U^{2}\right) U^{2}\right\} \operatorname{com} U \sim$ $S(0,1)$. Alguns valores de $d_{g}$ podem ser encontrados na Tabela 2.2.

No caso linear temos uma simplificação na função escore $U_{\beta}(\theta)$ e conseqüentemente no processo iterativo, visto que $\mathrm{D}_{\beta}=\mathrm{X}$. A função escore fica dada por $\mathrm{U}_{\beta}(\boldsymbol{\theta})=\frac{1}{\phi} \mathrm{X}^{T} \mathrm{D}(\mathrm{v})(\mathrm{y}-\mathrm{X} \boldsymbol{\beta})$ e o processo iterativo assume a forma

$$
\boldsymbol{\beta}^{(m+1)}=\left\{\mathrm{X}^{T} \mathbf{D}\left(\mathbf{v}^{(m)}\right) \mathbf{X}\right\}^{-1} \mathbf{X}^{T} \mathbf{D}\left(\mathbf{v}^{(m)}\right) \mathbf{y}
$$

e

$$
\phi^{(m+1)}=\frac{1}{n}\left\{\mathbf{y}-\mathbf{X} \boldsymbol{\beta}^{(m+1)}\right\}^{T} \mathbf{D}\left(\mathbf{v}^{(m+1)}\right)\left\{\mathbf{y}-\mathbf{X} \boldsymbol{\beta}^{(m+1)}\right\} \quad(m=0,1,2, \ldots) .
$$

Note que de (2.4) o peso $v_{i}^{(m)}$ é inversamente proporcional à distância entre o valor observado $y_{i}$ e o seu valor predito $\mu_{i}^{(m)}$ (a menos da normal que é uma 
Tabela 2.1 Expressões para $W_{g}(u)$ e $W_{g}^{\prime}(u)$ para algumas distribuições simétricas.

\begin{tabular}{|c|c|c|}
\hline Distribuição & $W_{g}(u)$ & $W_{g}^{\prime}(u)$ \\
\hline Normal & $-\frac{1}{2}$ & 0 \\
\hline$t$-Student & $-\frac{\nu+1}{2(\nu+u)}$ & $\frac{(\nu+1)}{2(\nu+u)^{2}}$ \\
\hline$t$-Student generalizada & $-\frac{(r+1)}{2(s+u)}$ & $\frac{(r+1)}{2(s+u)^{2}}$ \\
\hline Logística-I & $-\tanh \left(\frac{u}{2}\right)$ & $-\operatorname{sech}\left(\frac{u}{2}\right) / 2$ \\
\hline Logística-II & $-\frac{\exp (-\sqrt{u})-1}{(-2 \sqrt{u})[1+\exp (-\sqrt{u})]}$ & $\frac{2 \exp (-\sqrt{u}) \sqrt{u}+\exp (-2 \sqrt{u})-1}{-4 u^{3 / 2}[1+\exp (-\sqrt{u})]^{2}}$ \\
\hline Logística generalizada & $\frac{-\alpha m[\exp (-\alpha \sqrt{u})-1]}{(-2 \sqrt{u})[1+\exp (-\alpha \sqrt{u})]}$ & $-\frac{\alpha m}{4} \frac{2 \alpha \exp (-\alpha \sqrt{u}) \sqrt{u}+\exp (-2 \alpha \sqrt{u})-1}{u^{3 / 2}[1+\exp (-\alpha \sqrt{u})]^{2}}$ \\
\hline Exponencial potência & $-\frac{1}{2(1+k)(u)^{k /(k+1)}}$ & $\frac{k}{(1+k)^{2} 2 u^{(2 k+1) /(1+k)}}$ \\
\hline
\end{tabular}

função constante e da logística-I que é diretamente proporcional), de forma que observações mais distantes tendem a ter pesos menores no processo de estimação (veja discussão, por exemplo, em Lange, Little e Taylor, 1989). No caso linear e para a distribuição normal os estimadores de máxima verossimilhança tomam expressões em forma fechada, pois $v_{i}=1$, para todo $i$. Para a distribuição $t$-Student com $\nu$ graus de liberdade, temos que $g(u)=c(1+u / \nu)^{-(\nu+1) / 2}, \nu>0$ e $u>0$ de forma que $W_{g}\left(u_{i}\right)=-(\nu+1) / 2\left(\nu+u_{i}\right)$ e $v_{i}=(\nu+1) /\left(\nu+u_{i}\right)$, para todo $i$. Para a distribuição exponencial potência com parâmetro de forma $\gamma=1 /(1+k)$ fixado, $g(u)=c e^{-0,5 u^{\gamma-1}}, u>0$ e $\gamma \geqslant 1 / 2$, então $W_{g}\left(u_{i}\right)=-\frac{1}{2} \gamma u_{i}^{\gamma-1}$ e $v_{i}=\gamma u_{i}^{\gamma-1}$.

\subsubsection{Informação de Fisher}

Seja $-\left.\ddot{\mathbf{L}}_{\theta \theta}\right|_{\hat{\theta}}$ a matriz de informação observada de Fisher para $\theta$. Depois de algumas manipulações algébricas, encontramos o seguinte :

$$
\ddot{\mathrm{L}}_{\theta \theta}=\left[\begin{array}{cc}
\ddot{\mathrm{L}}_{\beta \beta} & \ddot{\mathrm{L}}_{\beta \phi} \\
\ddot{\mathrm{L}}_{\phi \beta} & \ddot{\mathrm{L}}_{\phi \phi}
\end{array}\right], \quad \text { em que }
$$


MODELO SIMÉTRICO DE REGRESSÃO

$$
\begin{aligned}
\ddot{\mathrm{L}}_{\beta \beta} & =-\frac{1}{\phi}\left\{\sum_{i=1}^{n} 2 s_{i} \mathbf{D}_{\beta \beta}(i)+\mathbf{D}_{\beta}^{T} \mathrm{D}(\mathbf{a}) \mathbf{D}_{\beta}\right\} \\
& =-\frac{1}{\phi}\left\{\left[2 \mathrm{~s}^{T}\right]\left[\mathbf{D}_{\beta \beta}\right]+\mathbf{D}_{\beta}^{T} \mathbf{D}(\mathbf{a}) \mathbf{D}_{\beta}\right\}, \\
\ddot{\mathrm{L}}_{\beta \phi} & =\frac{2}{\phi^{2}} \mathbf{D}_{\beta}^{T} \mathrm{~b} \mathrm{e} \\
\ddot{\mathrm{L}}_{\phi \phi} & =\frac{1}{\phi^{2}}\left\{\frac{n}{2}+\mathbf{u}^{T} \mathbf{D}(\mathrm{c}) \mathbf{u}-\frac{1}{\phi} \mathrm{e}^{T} \mathrm{D}(\mathrm{v}) \mathrm{e}\right\},
\end{aligned}
$$

sendo $\mathbf{D}_{\beta \beta}(i)=\partial^{2} \mu_{i} / \partial \boldsymbol{\beta} \partial \boldsymbol{\beta}^{T}, \mathbf{D}(\mathbf{a})=\operatorname{diag}\left\{a_{1}, \ldots, a_{n}\right\}, \mathbf{D}(\mathbf{c})=\operatorname{diag}\left\{c_{1}, \ldots, c_{n}\right\}$, $\mathbf{b}^{T}=\left(b_{1}, \ldots, b_{n}\right), \mathbf{u}=\left(u_{1}, \ldots, u_{n}\right)^{T}, a_{i}=v_{i}-4 W_{g}^{\prime}\left(u_{i}\right) u_{i}, c_{i}=W_{g}^{\prime}\left(u_{i}\right), b_{i}=$ $\left\{W_{g}\left(u_{i}\right)+u_{i} W_{g}^{\prime}\left(u_{i}\right)\right\} e_{i}, e_{i}=\left(y_{i}-\mu_{i}\right), s_{i}=W_{g}\left(u_{i}\right) e_{i}, i=1, \ldots, n$ e a notação entre colchetes está definida no Apêndice A. No caso linear temos que $\mathbf{D}_{\beta \beta}(i)=0$, para todo $i$, coincidindo com as expressões dadas em Galea, Paula e Uribe-Opazo (2003).

Tabela 2.2 Valores de $d_{g}, f_{g}$ e $\xi$ para algumas distribuições simétricas.

\begin{tabular}{r|c|c|c}
\hline Distribuição & $d_{g}$ & $f_{g}$ & $\xi$ \\
\hline Normal & $\frac{1}{4}$ & $\frac{3}{4}$ & 1 \\
$t$-Student & $\frac{(\nu+1)}{4(\nu+3)}$ & $\frac{3(\nu+1)}{4(\nu+3)}$ & $\frac{\nu}{\nu-2}, \quad \nu>2$ \\
$t$-Student generalizada & $\frac{r(r+1)}{4 s(r+3)}$ & $\frac{3(r+1)}{4(r+3)}$ & $\frac{s}{r-2}, \quad s>0, r>2$ \\
Logística-I & 0,369310044 & 1,003445984 & 0,79569 \\
Logística-II & $\frac{1}{12}$ & 0,60749 & $\pi^{2} / 3$ \\
Logística generalizada & $\frac{\alpha^{2} m^{2}}{4(2 m+1)}$ & $\frac{2 m\left(2+m^{2} \psi^{\prime}(m)\right)}{4(2 m+1)}$ & $2 \psi^{\prime}(m)$ \\
Exponencial potência & $\frac{\Gamma\{(3-k) / 2\}}{4\left(2^{k-1}\right)(1+k)^{2} \Gamma\{(k+1) / 2\}}$ & $\frac{(k+3)}{4(k+1)}$ & $2^{(1+k)} \frac{\Gamma\{3(k+1) / 2\}}{\Gamma\{(k+1) / 2\}}$ \\
\hline
\end{tabular}


A inversa de $\ddot{\mathrm{L}}_{\theta \theta}$ pode ser expressa na forma

$$
\ddot{\mathrm{L}}_{\theta \theta}^{-1}=\left[\begin{array}{cc}
-\phi \mathrm{M}^{-1}+\frac{\mathrm{AA}^{T}}{\mathrm{E}} & \frac{\mathrm{A}}{\mathrm{E}} \\
\frac{\mathrm{A}^{T}}{\mathrm{E}} & \frac{1}{\mathrm{E}}
\end{array}\right],
$$

em que $\mathbf{M}=2\left[\mathbf{s}^{T}\right]\left[\mathbf{D}_{\beta \beta}\right]+\mathbf{D}_{\beta}^{T} \mathbf{D}(\mathbf{a}) \mathbf{D}_{\beta}, \mathbf{A}=\frac{2}{\phi} \mathbf{M}^{-1} \mathbf{D}_{\beta}^{T} \mathbf{b}$ e $\mathrm{E}=\ddot{\mathbf{L}}_{\phi \phi}+\frac{2}{\phi^{2}} \mathbf{b}^{T} \mathbf{D}_{\beta} \mathbf{A}$.

A matriz de informação esperada de Fisher para $\theta$ pode ser expressa na forma

$$
\mathrm{K}_{\theta \theta}=\left[\begin{array}{cc}
\mathrm{K}_{\beta \beta} & 0 \\
0 & \mathrm{~K}_{\phi \phi}
\end{array}\right]
$$

em que $\mathbf{K}_{\beta \beta}=\frac{4 d_{g}}{\phi} \mathbf{D}_{\beta}^{T} \mathrm{D}_{\beta}$ com $\mathrm{K}_{\phi \phi}=\frac{n}{4 \phi^{2}}\left(4 f_{g}-1\right), f_{g}=\mathrm{E}\left\{W_{g}^{2}\left(U^{2}\right) U^{4}\right\}$ e $U \sim S(0,1)$ (veja Tabela 2.2). Portanto, temos ortogonalidade entre $\beta$ e $\phi$. Por exemplo, para a distribuição $t$-Student com $\nu$ graus de liberdade temos que $d_{g}=(\nu+1) /\{4(\nu+3)\}$ e $f_{g}=3(\nu+1) /\{4(\nu+3)\}$.

Assumimos que $\beta \in \Omega_{\beta} \subset \mathbb{R}^{p}$, em que $\Omega_{\beta}$ é um conjunto aberto com pontos interiores. É possível mostrar que $\hat{\boldsymbol{\beta}}$, o estimador de máxima verossimilhança de $\boldsymbol{\beta}$, é um estimador consistente de $\boldsymbol{\beta}$, e

$$
\sqrt{n}(\hat{\boldsymbol{\beta}}-\boldsymbol{\beta}) \stackrel{\mathrm{d}}{\rightarrow} \mathrm{N}_{p}\left(\mathbf{0}, \mathbf{J}_{\beta \beta}^{-1}\right), \text { em que } \mathbf{J}_{\beta \beta}=\lim _{n \rightarrow \infty} \frac{1}{n} \mathbf{K}_{\beta \beta}
$$

Então, $\hat{\mathbf{K}}_{\beta \beta}^{-1}=\frac{\hat{\phi}}{4 d_{g}}\left(\mathbf{D}_{\hat{\beta}}^{T} \mathbf{D}_{\hat{\beta}}\right)^{-1}$ é um estimador consistente da matriz de variânciacovariância assintótica de $\hat{\boldsymbol{\beta}}$. Observe que no caso linear a matriz de correlação assintótica não depende de parâmetros desconhecidos. De forma similar, $\hat{\phi}$, o estimador de máxima verossimilhança de $\phi$, é um estimador consistente de $\phi$, e

$$
\sqrt{n}(\hat{\phi}-\phi) \stackrel{\mathrm{d}}{\rightarrow} \mathrm{N}\left(0, \mathrm{~J}_{\phi \phi}^{-1}\right), \quad \text { em que } \quad \mathrm{J}_{\phi \phi}=\lim _{n \rightarrow \infty} \frac{1}{n} \mathrm{~K}_{\phi \phi}
$$

Então, $\hat{\mathrm{K}}_{\phi \phi}^{-1}=\frac{4 \hat{\phi}^{2}}{n\left(4 f_{g}-1\right)}$ é um estimador consistente da variância assintótica de $\hat{\phi}$.

\subsection{Resíduos}

Uma pergunta comum após o ajustamento de um modelo sugerido é : "será que o modelo se ajusta bem aos dados ?" É importante responder a essa pergunta pois se o modelo não estiver bem ajustado, o mesmo pode fornecer conclusões errôneas. 
Uma técnica que pode ajudar a responder essa pergunta é a análise de resíduos. Essa técnica verifica, por exemplo, se há afastamentos sérios das suposições feitas para os erros e se existem observações aberrantes. Uma definição natural de resíduo é a diferença entre a resposta observada e o valor predito, denominado resíduo ordinário. É importante conhecer algumas propriedades desse resíduo. Nesse sentido, podemos utilizar a metodologia apresentada em Cox e Snell (1968) para determinar os momentos do resíduo ordinário em modelos simétricos. Consideraremos o resíduo ordinário com $\phi$ conhecido ou fixo expresso na forma abaixo

$$
r_{i}\left(y_{i}, \hat{\mu}_{i}, \phi\right)=y_{i}-\hat{\mu}_{i}, \quad i=1, \ldots, n
$$

em que $\mu_{i}=\mu\left(\mathrm{x}_{i}, \boldsymbol{\beta}\right), y_{i}=\mu_{i}+\epsilon_{i}$ e $\epsilon_{i} \sim S(0, \phi)$.

Esses resíduos são, em geral, viesados e têm distribuição não normal, mesmo assintoticamente, dificultando a verificação da adequacidade dos modelos pelos métodos tradicionais. Em modelos de regressão normais não-lineares Cook e Tsai (1985) propuseram o resíduo projetado obtido num sub-espaço dos resíduos ordinários. Esses novos resíduos têm distribuição aproximadamente normal de média zero e variância dependendo de $\sigma^{2}$. Contudo, árduas álgebras podem ser necessárias para obter tais resíduos.

Propomos a seguir corrigir, até ordem $n^{-1}$, os dois primeiros momentos de $r_{i}$ a fim de obtermos propriedades próximas às do $i$-ésimo erro $\epsilon_{i}=y_{i}-\mu_{i}$. A expansão em série de Taylor de $r_{i}-\epsilon_{i}$, sendo $r_{i}=h\left(y_{i}, \hat{\boldsymbol{\beta}}, \phi\right)$ e $\epsilon_{i}=h\left(y_{i}, \boldsymbol{\beta}, \phi\right)$ em termos de $\hat{\beta}_{r}-\beta_{r}, r=1, \ldots, p$, até ordem $n^{-1}$, é dada pela seguinte expressão :

$$
r_{i}=\epsilon_{i}+\left(\hat{\beta}_{r}-\beta_{r}\right) H_{r}^{i}+\frac{1}{2}\left(\hat{\beta}_{r}-\beta_{r}\right)\left(\hat{\beta}_{s}-\beta_{s}\right) H_{r s}^{i}
$$

sendo

$$
H_{r}^{i}=\frac{\partial h\left(y_{i}, \boldsymbol{\beta}, \phi\right)}{\partial \beta_{r}}=\frac{\partial h\left(y_{i}, \boldsymbol{\beta}, \phi\right)}{\partial \mu_{i}} \frac{\partial \mu_{i}}{\partial \beta_{r}}
$$

e

$$
H_{r s}^{i}=\frac{\partial^{2} h\left(y_{i}, \boldsymbol{\beta}, \phi\right)}{\partial \beta_{r} \partial \beta_{s}}=\frac{\partial h\left(y_{i}, \boldsymbol{\beta}, \phi\right)}{\partial \mu_{i}} \frac{\partial^{2} \mu_{i}}{\partial \beta_{r} \partial \beta_{s}} .
$$

Seja $h\left(y_{i}, \boldsymbol{\beta}, \phi\right)=y_{i}-\mu_{i}$, então temos por (2.10) e (2.11) que $H_{r}^{i}=-d_{i r}$ e $H_{r s}^{i}=$ $-g_{i r s}$ em que $\frac{\partial \mu_{i}}{\partial \beta_{r}}=d_{i r}$ e $\frac{\partial^{2} \mu_{i}}{\partial \beta_{r} \partial \beta_{s}}=g_{i r s}$. 
Assim, aplicando a esperança na expressão (2.9) e procedendo de forma análoga a Cox e Snell (1968), obtemos

$$
\begin{aligned}
& \mathrm{E}\left(r_{i}\right)=\mathrm{E}\left(\epsilon_{i}\right)+\mathrm{E}\left(\hat{\beta}_{r}-\beta_{r}\right) \mathrm{E}\left(H_{r}^{i}\right)+I^{r s} \mathrm{E}\left(H_{r}^{i} U_{s}^{i}+\frac{1}{2} H_{r s}^{i}\right) \\
& \mathrm{E}\left(r_{i}\right)=\mathrm{E}\left(\epsilon_{i}\right)+\mathcal{A}_{i},
\end{aligned}
$$

sendo que no lado direito de (2.12) aplica-se a convenção da soma para $r, s=$ $1, \ldots, p, \epsilon_{i}=h\left(y_{i}, \boldsymbol{\beta}, \phi\right)=y_{i}-\mu_{i}, \mathrm{E}\left(\hat{\beta}_{r}-\beta_{r}\right)$ é o viés de ordem $n^{-1}$, dado no Apêndice A (A.5). $U_{s}^{i}$ é a função escore $\frac{\partial \mathrm{L}(\boldsymbol{\theta})}{\partial \beta_{s}}=-\frac{2}{\phi} W_{g}\left(u_{i}\right)\left(y_{i}-\mu_{i}\right) d_{i s}, I_{r s}$ é $(r, s)$-ésimo elemento da matriz $\mathbf{K}_{\beta \beta}=\frac{4 d_{g}}{\phi} \sum_{i=1}^{n} d_{i r} d_{i s}$ e $I^{r s}$ é o $(r, s)$-elemento da matriz $\mathbf{K}_{\beta \beta}^{-1}$. Aplicando esperança obtemos

$$
\begin{aligned}
\mathrm{E}\left(\epsilon_{i}\right) & =0 \\
\mathrm{E}\left(H_{r}^{i}\right) & =-d_{i r}, \\
\mathrm{E}\left(H_{r s}^{i}\right) & =-g_{i r s} \mathrm{e} \\
\mathrm{E}\left(H_{r}^{i} U_{s}^{i}\right) & =\mathrm{E}\left\{\frac{2}{\phi} W_{g}\left(u_{i}\right)\left(y_{i}-\mu_{i}\right) d_{i r} d_{i s}\right\}=0, \quad \forall(r, s) .
\end{aligned}
$$

De (2.15) e (2.16) temos que

$$
I^{r s} \mathrm{E}\left(H_{r}^{i} U_{s}^{i}+\frac{1}{2} H_{r s}^{i}\right)=-\frac{1}{2} I^{r s} g_{i r s} .
$$

Assumindo que a convenção da soma é dada para os índices $r, s=1, \ldots, p$ e substituindo (2.13),(2.14), (A.5) e (2.17) em (2.12) obtemos até ordem $n^{-1}$

$$
\mathrm{E}\left(r_{i}\right)=-\mathrm{d}_{i}^{T}\left(\mathbf{D}_{\beta}^{T} \mathbf{D}_{\beta}\right)^{-1} \mathbf{D}_{\beta}^{T} \eta+\eta_{i},
$$

em que $\eta=\left(\eta_{1}, \ldots, \eta_{n}\right)^{T}, \eta_{i}=-\frac{\phi}{8 d_{g}} \operatorname{tr}\left\{\left(\mathbf{D}_{\beta}^{T} \mathbf{D}_{\beta}\right)^{-1} \mathbf{D}_{\beta \beta}(i)\right\}$ e $\mathbf{d}_{i}=\left(d_{i 1}, \ldots, d_{i p}\right)^{T}$. Conseqüentemente, em forma matricial

$$
\mathrm{E}(\mathrm{r})=\left(\mathrm{I}_{n}-\mathbf{H}\right) \eta
$$

em que $\mathbf{H}=\mathbf{D}_{\beta}\left(\mathbf{D}_{\beta}^{T} \mathbf{D}_{\beta}\right)^{-1} \mathbf{D}_{\beta}^{T}$ e $\mathbf{I}_{n}$ é a matriz identidade de ordem $n$, generalizando as expressões dadas em Cook, Tsai e Wei (1986) que encontraram essa relação para os modelos normais não-lineares. 
Vamos calcular agora $\mathrm{E}\left(r_{i}^{2}\right)$. Assim,

$$
\mathrm{E}\left(r_{i}^{2}\right)=\mathrm{E}\left(\epsilon_{i}^{2}\right)+2 \mathrm{E}\left(\hat{\beta}_{r}-\beta_{r}\right) \mathrm{E}\left(\epsilon_{i} H_{r}^{i}\right)+2 I^{r s} \mathrm{E}\left(\epsilon_{i} H_{r}^{i} U_{s}^{i}+\frac{1}{2} H_{r}^{i} H_{s}^{i}+\frac{1}{2} \epsilon_{i} H_{r s}^{i}\right),
$$

$\operatorname{com} \epsilon_{i}^{2}=\left(y_{i}-\mu_{i}\right)^{2}, \epsilon_{i} H_{r}^{i}=-\left(y_{i}-\mu_{i}\right) d_{i r}, \epsilon_{i} H_{r}^{i} U_{s}^{i}=2 W_{g}\left(u_{i}\right)\left(u_{i}\right) d_{i r} d_{i s}, \frac{1}{2} H_{r}^{i} H_{s}^{i}=$ $\frac{1}{2} d_{i r} d_{i s}$ e $\frac{1}{2} \epsilon_{i} H_{r s}^{i}=-\frac{1}{2}\left(y_{i}-\mu_{i}\right) g_{i r s}$.

Aplicando esperança obtemos

$$
\begin{aligned}
\mathrm{E}\left(\epsilon_{i}^{2}\right) & =\xi \phi, \\
\mathrm{E}\left(\epsilon_{i} H_{r}^{i}\right) & =0 \quad \mathrm{e} \\
\mathrm{E}\left(\frac{1}{2} \epsilon_{i} H_{r s}^{i}\right) & =0 .
\end{aligned}
$$

De Fang, Kotz e $\mathrm{Ng}\left(1990\right.$, p.94) segue que $\mathrm{E}\left\{W_{g}(u) u\right\}=-1 / 2$, em que $\sqrt{u} \sim$ $S(0,1)$. Logo,

$$
\mathrm{E}\left(\epsilon_{i} H_{r}^{i} U_{s}^{i}\right)=-d_{i r} d_{i s}
$$

Substituindo (2.20)-(2.23) em (2.19), obtemos até ordem $n^{-1}$

$$
\mathrm{E}\left(r_{i}^{2}\right)=\xi \phi-I^{r s} d_{i r} d_{i s}
$$

e

$$
\begin{aligned}
\operatorname{Var}\left(r_{i}\right) & =\xi \phi\left\{1-\left(4 d_{g} \xi\right)^{-1} a_{r s} d_{i r} d_{i s}\right\} \\
& =\operatorname{Var}\left(y_{i}\right)\left\{1-\left(4 d_{g} \xi\right)^{-1} h_{i i}\right\} \\
& =\phi \xi\left\{1-\left(4 d_{g} \xi\right)^{-1} h_{i i}\right\},
\end{aligned}
$$

em que $a_{r s}$ é o $(r, s)$-ésimo elemento da matriz $\left(\mathbf{D}_{\beta}^{T} \mathbf{D}_{\beta}\right)^{-1}$ e $h_{i i}=\mathbf{d}_{i}^{T}\left(\mathbf{D}_{\beta}^{T} \mathbf{D}_{\beta}\right)^{-1} \mathbf{d}_{i}$.

Para $\operatorname{Cov}\left(r_{i}, r_{j}\right)$ temos que

$$
\operatorname{Cov}\left(r_{i}, r_{j}\right)=\mathrm{E}^{2}\left(\epsilon_{i}\right)+\left(\mathcal{A}_{i}+\mathcal{A}_{j}\right) \mathrm{E}\left(\epsilon_{i}\right)+I^{r s} \mathrm{E}\left(\epsilon_{i} H_{r}^{j} U_{s}^{i}+\epsilon_{j} H_{r}^{i} U_{s}^{j}+H_{r}^{i} H_{s}^{j}\right),
$$

em que $\epsilon_{i} H_{r}^{j} U_{s}^{i}=2 W_{g}\left(u_{i}\right) u_{i} d_{j r} d_{i s}$ e $\epsilon_{j} H_{r}^{i} U_{s}^{j}=2 W_{g}\left(u_{j}\right) u_{j} d_{i r} d_{j s}$. Além disso,

$$
\begin{aligned}
\mathrm{E}\left(\epsilon_{i} H_{r}^{j} U_{s}^{i}\right) & =-d_{j r} d_{i s}, \\
\mathrm{E}\left(\epsilon_{j} H_{r}^{i} U_{s}^{j}\right) & =-d_{i r} d_{j s} \quad \mathrm{e} \\
\mathrm{E}\left(H_{r}^{i} H_{s}^{j}\right) & =d_{i r} d_{j s} .
\end{aligned}
$$


RESíDUOS

Substituindo as equações (2.26)-(2.28) em (2.25) e desde que $\mathrm{E}\left(\epsilon_{i}\right)=0$, segue que

$$
\mathrm{E}\left(r_{i}, r_{j}\right)=-I^{r s} d_{j r} d_{j s}
$$

Sendo assim,

$$
\begin{aligned}
\operatorname{Cov}\left(r_{i}, r_{j}\right) & =-\frac{\phi}{4 d_{g}} a_{r s} d_{j r} d_{i s} \\
& =-\phi \xi\left(4 d_{g} \xi\right)^{-1} h_{i j}, \quad i \neq j,
\end{aligned}
$$

em que $h_{i j}=\mathrm{d}_{i}^{T}\left(\mathbf{D}_{\beta}^{T} \mathbf{D}_{\beta}\right)^{-1} \mathrm{~d}_{j}$. Portanto, em notação matricial temos que a matriz de variância-covariância do vetor de resíduos ordinários pode ser expressa na forma

$$
\operatorname{Var}(\mathbf{r})=\phi \xi\left\{\mathbf{I}_{n}-\left(4 d_{g} \xi\right)^{-1} \mathbf{H}\right\}
$$

em que $\mathbf{I}_{n}$ é a matriz identidade de ordem $n$ e $\mathbf{H}$ é uma matriz $n \times n$ com $(i, j)$-ésimo elemento dado por $h_{i j}$.

No caso em que podemos estabelecer uma relação linear nos parâmetros, $\mu_{i}=$ $\mathrm{x}_{i}^{T} \boldsymbol{\beta}$, encontramos simplicações interessantes nas expressões acima. Devido ao fato de que o viés de ordem $n^{-1}$ de $\hat{\boldsymbol{\beta}}$ é nulo quando temos um relação linear nos parâmetros e que $H_{r s}^{i}=0 \forall(r, s)$ e $i=1, \ldots, n$ segue o seguinte :

$$
\mathrm{E}(\mathrm{r})=0 \quad \text { e } \quad \operatorname{Var}(\mathbf{r})=\phi \xi\left\{\mathbf{I}_{n}-\left(4 d_{g} \xi\right)^{-1} \mathbf{H}\right\}
$$

em que $\mathrm{H}=\mathrm{X}\left(\mathrm{X}^{T} \mathrm{X}\right)^{-1} \mathrm{X}^{T}$.

Como os $r_{i}$ 's têm variâncias diferentes, é conveniente expressá-los em forma padronizada, a fim de permitir uma comparabilidade entre os mesmos. Uma definição natural do resíduo padronizado é subtrair pela média e dividir pelo respectivo desvio-padrão, obtendo a expressão

$$
\begin{aligned}
t_{r_{i}} & =\frac{r_{i}}{\{\xi \hat{\phi}\}^{1 / 2}\left\{1-\left(4 d_{g} \xi\right)^{-1} \hat{h}_{i i}\right\}^{1 / 2}} \\
& =\frac{y_{i}-\hat{y}_{i}}{\{\xi \hat{\phi}\}^{1 / 2}\left\{1-\left(4 d_{g} \xi\right)^{-1} \hat{h}_{i i}\right\}^{1 / 2}}, \quad i=1, \ldots, n .
\end{aligned}
$$

Estudos de simulação indicam que o resíduo proposto acima tem média e variância aproximadamente zero e um, respectivamente, uma assimetria desprezível e uma curtose acompanhando a curtose da distribuição do erro (veja, por exemplo, Tabela $2.3)$. 
Tabela 2.3 Análise descritiva para o resíduo $t_{r_{i}}$ de 1000 observações geradas do modelo ajustado na Seção 2.4.

\begin{tabular}{r|ccc}
\hline Estatística & Normal & $t_{10}$ & Logístico-II \\
\hline média & $-0,000$ & $-0,002$ & $-0,000$ \\
variância & 1,044 & 1,041 & 1,037 \\
assimetria & 0,003 & $-0,029$ & $-0,008$ \\
curtose & $-0,012$ & 0,862 & 1,035 \\
\hline
\end{tabular}

\subsection{Coelhos europeus na Austrália}

Para ilustrar uma aplicação consideraremos o conjunto de dados descrito em Ratkowsky (1983, Tabela 6.1) apresentado no Apêndice C, cujo interesse principal é relacionar o peso das lentes dos olhos de coelhos europeus, $y$ (em mg) (Oryctolagus cuniculus) e a idade do animal, $x$ (em dias), em uma amostra de 71 observações. Esse animal é largamente distribuído na população selvagem da Austrália. Um aspecto interessante para esse conjunto de dados, que suporta o uso de erros com distribuição com caudas mais pesadas que a normal, é a suspeita de dois pontos aberrantes sob estimação de mínimos quadrados. Então, para reanalizar o dados, propomos o seguinte modelo :

$$
y_{i}=\exp \left(\alpha-\frac{\beta}{x_{i}+\gamma}\right) e^{\epsilon_{i}}, \quad i=1, \ldots, 71
$$

em que $\epsilon_{i} \sim \mathrm{S}(0, \phi)$ são erros mutuamente independentes.

Várias distribuições com caudas mais pesadas do que a normal foram assumidas, porém, somente dois modelos parecem ajustar-se aos dados tão bem quanto ou melhor do que o modelo normal, o modelo $t$-Student com 10 graus de liberdade e o modelo logístico-II. Os graus de liberdade do modelo $t$-Student foram estimados pelo método dos momentos. O coeficiente de afastamento da curtose para esses dois modelos são, respectivamente, $\gamma_{2}=1$ e $\gamma_{2}=1,2$.

A Figura 2.1 indica que a variabilidade da resposta cresce quando a idade do 
Figura 2.1 Gráfico de dispersão do peso das lentes dos olhos contra idade de coelhos europeus.

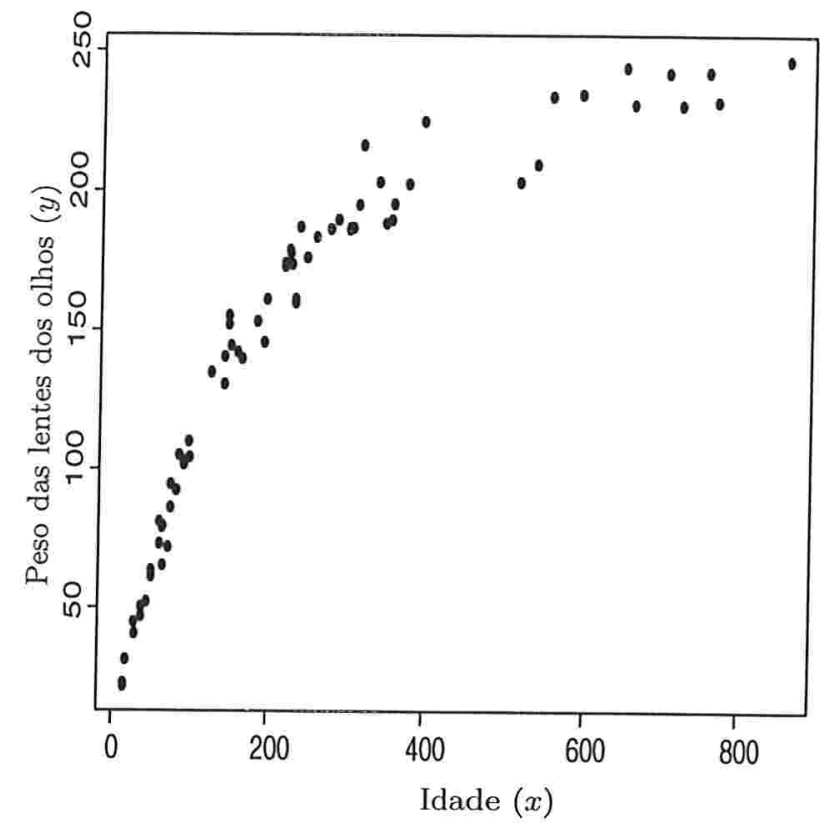

animal cresce, justificando o uso de um modelo multiplicativo. As estimativas de máxima verossimilhança são apresentadas na Tabela 2.4, as quais em geral são parecidas, embora os erros padrões das estimativas dos modelos $t$-Student e logístico-II são, em geral, menores do que as estimativas dos erros padrões do modelo normal. A curvatura intrínseca e paramétrica são desprezíveis nos três modelos, e o viés relativo das estimativas dos parâmetros tende a ser menor nos modelos com curtose maior (veja Tabela 2.5). Além disso, os gráficos de resíduos contra os valores ajustados mostram que as observações 4, 5, 16 e 17 aparecem com destaque em todos os modelos ajustados (veja Figuras $2.2-2.4$ ). Os gráficos normais de probabilidades com envelope para o resíduo $t_{r_{i}}$ não apresentam nenhum comportamento não usual (Figuras $2.2-2.4$ ). No capítulo 4 voltaremos a discutir esse exemplo, no qual, baseado em métodos de validação, escolheremos dentre esses modelos o que melhor se adequada aos dados. 
Tabela 2.4 Estimativas de máxima verossimilhança (erro padrão aproximado) para alguns modelos simétricos ajustados aos dados dos coelhos da Tabela C.1.

\begin{tabular}{c|ccc}
\hline Parâmetro & Normal & $t_{10}$ & Logístico-II \\
\hline$\alpha$ & 5,640 & 5,633 & 5,633 \\
& $(0,020)$ & $(0,018)$ & $(0,018)$ \\
$\beta$ & 130,583 & 127,540 & 127,258 \\
& $(5,603)$ & $(5,097)$ & $(4,992)$ \\
$\gamma$ & 37,603 & 36,079 & 35,864 \\
& $(2,273)$ & $(2,061)$ & $(2,016)$ \\
$\phi$ & 0,004 & 0,003 & 0,001 \\
& $(0,0006)$ & $(0,0005)$ & $(0,0002)$ \\
\hline
\end{tabular}

Tabela 2.5 Medidas de não-linearidade e viés relativo das estimativas dos parâmetros de locação para alguns modelos simétricos ajustados aos dados dos coelhos da Tabela C.1.

\begin{tabular}{c|ccc}
\hline Parâmetro & Normal & $t_{10}$ & Logístico-II \\
\hline$\% \mathrm{~B}(\hat{\beta})$ & 0,005 & 0,004 & 0,004 \\
$\% \mathrm{~B}(\hat{\alpha})$ & 0,115 & 0,100 & 0,096 \\
$\% \mathrm{~B}(\hat{\gamma})$ & 0,150 & 0,133 & 0,128 \\
$\gamma^{P E}$ & 0,080 & 0,073 & 0,072 \\
$\gamma^{I N}$ & 0,021 & 0,019 & 0,019 \\
\hline
\end{tabular}


Figura 2.2 Gráfico normal de probabilidades com envelope para $t_{r_{i}}$ (esquerda) $e$ gráfico de resíduos $t_{r_{i}}$ contra os valores ajustados para o modelo normal (direita) ajustado aos dados dos coelhos da Tabela C.1.
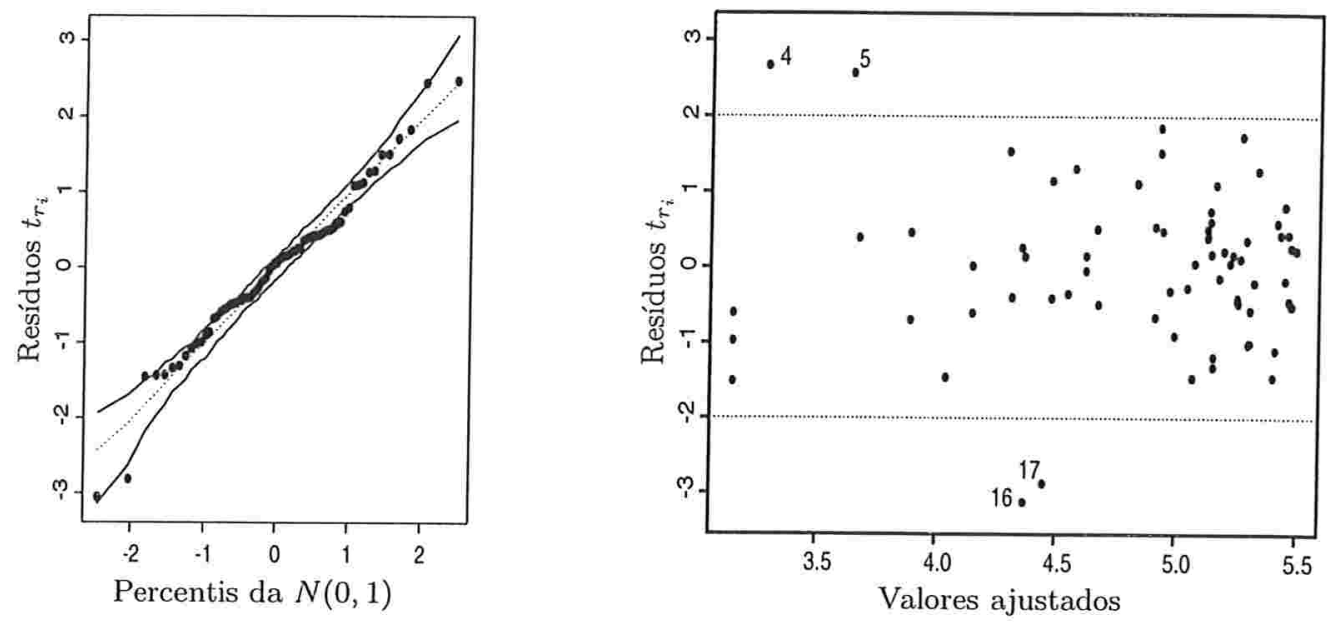

Figura 2.3 Gráfico normal de probabilidades com envelope para $t_{r_{i}}$ (esquerda) $e$ gráfico de resíduos $t_{r_{i}}$ contra os valores ajustados para o modelo $t$-Student com 10 g.l. (direita) ajustado aos dados dos coelhos da Tabela C.1.
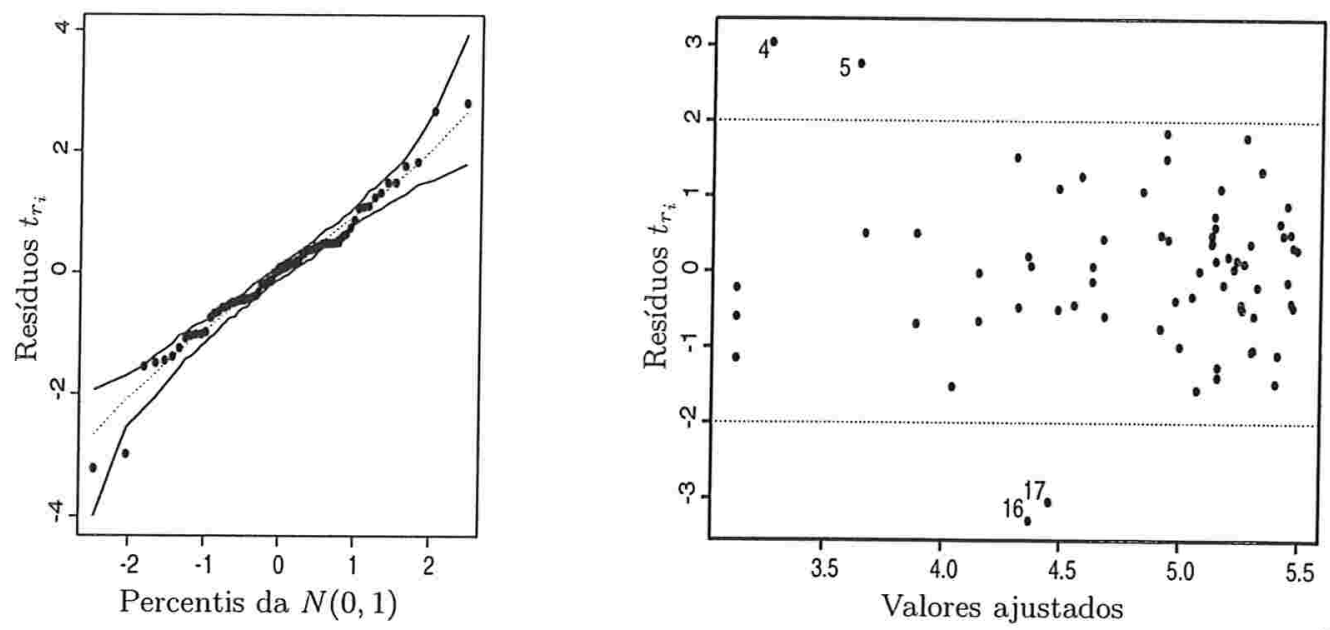
Figura 2.4 Gráfico normal de probabilidades com envelope para $t_{r_{i}}$ (esquerda) $e$ gráfico de resíduos $t_{r_{i}}$ contra os valores ajustados para o modelo logístico-II (direita) ajustado aos dados dos coelhos da Tabela C.1.
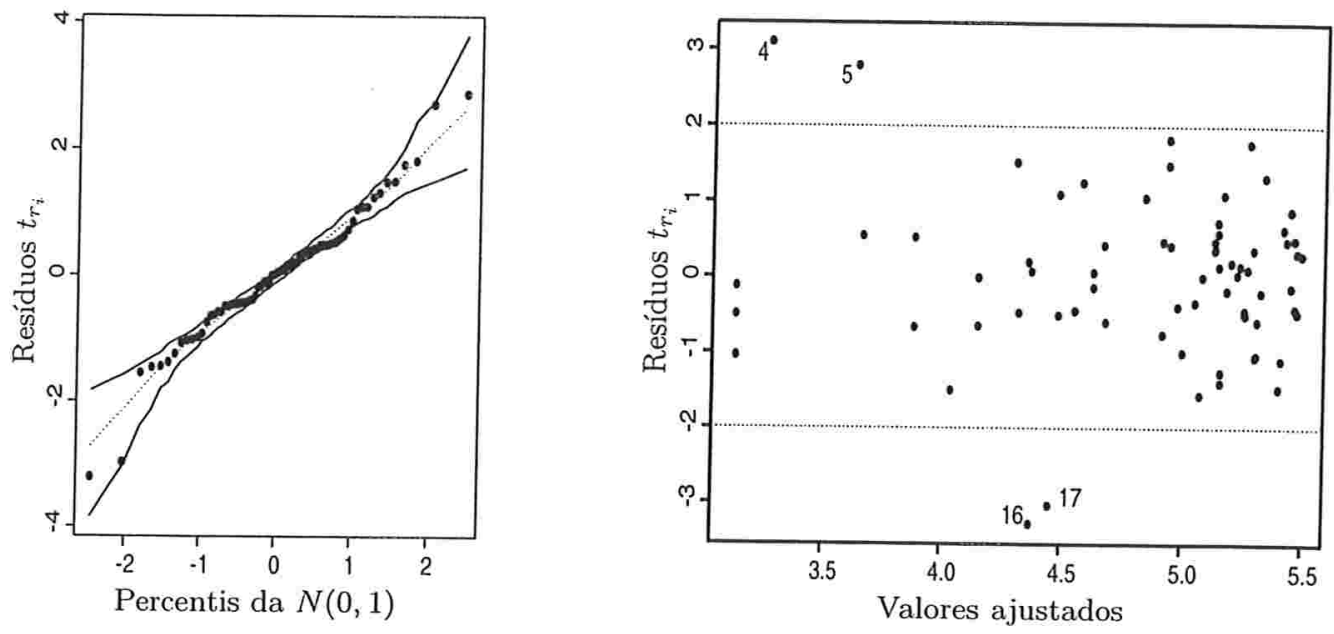


\section{CAPÍTULO 3}

\section{Modelos simétricos lineares heteroscedásticos}

\subsection{Introdução}

A modelagem de dados simétricos é, frequentemente, baseada na suposição de variância constante para os erros. Contudo, em muitas situações práticas essa suposição é dificilmente verificada. A procura de uma transformação na variável resposta para estabilizar a variância, nem sempre tem seu sucesso alcançado ou mesmo é recomendável. Trataremos, neste capítulo, de modelos simétricos de regressão, em que, um parâmetro de dispersão é atribuído para cada observação, sendo relacionado linearmente através de combinações lineares de variáveis explanatórias, por meio de uma função de ligação conhecida. A modelagem da variância tem sido largamente discutida principalmente na área de econometria. Park (1966) propôs um processo de estimação em 2-estágios para modelos log-lineares para a variância e Harvey (1976) tratou de modelos mais gerais. Para verificar a presença de heteroscedasticidade foram desenvolvidos diversos testes de hipótese (por exemplo, Ascombe, 1961; Bickel, 1978). Sob erros normais, por exemplo, Cook e Weisberg (1983) e Atkinson (1985) apresentam alguns métodos gráficos para detectar heteroscedasticidade. Importante passo foi dado por Aitkin (1987) que desenvolveu rotinas computacionais no GLIM para a estimação de máxima verossimilhança para modelagem da variância sob erros normais. Carroll e Ruppert (1988) desenvolveram procedimentos de diagnóstico usando métodos de influência local para as estimativas dos parâmetros da variância em vários modelos não-lineares para a média, enquanto que Verbyla (1993) compara as estimativas de máxima verossimilhança completa e residual baseando-se na deleção de casos e no afastamento da verossimilhança. Symth (1989) descreve um método que permite a modelagem do parâmetro de dispersão em alguns modelos lineares generalizados e, também, para 
modelos de quase-verossimilhança. Vasconcellos, Cordeiro e Barroso (2000) obtiveram expressões para o viés de segunda ordem e sua versão corrigida das estimativas dos parâmetros em modelos heteroscedásticos com erros $t$-Student. Barroso, Cordeiro e Vasconcellos (2002) obtiveram um fator de correção tipo-Bartlett para o teste escore para modelos de regressão heteroscedásticos com erros $t$-Student.

\subsection{Modelos simétricos lineares heteroscedásticos}

Considere o modelo simétrico de regressão linear

$$
y_{i}=\mu_{i}+\sqrt{\phi_{i}} \epsilon_{i}, \quad i=1, \ldots, n
$$

sendo a densidade de $y_{i}$ dada por

$$
f_{y_{i}}(y)=\frac{1}{\sqrt{\phi_{i}}} g\left\{\left(y_{i}-\mu_{i}\right)^{2} / \phi_{i}\right\}
$$

em que $y_{1}, \ldots, y_{n}$ são variáveis respostas observadas, $\mu_{i}=\mathbf{x}_{i}^{T} \boldsymbol{\beta}$ em que $\mathbf{x}_{i}=$ $\left(x_{i 1}, \ldots, x_{i p}\right)^{T}$ contém valores de $p$ variáveis explanatórias, $\beta=\left(\beta_{1}, \ldots, \beta_{p}\right)^{T}$ e $\epsilon_{i} \sim \mathrm{S}(0,1)$. Assumimos que o parâmetro de dispersão $\phi_{i}$ é parametrizado tal que $\phi_{i}=h_{i}=h\left(\tau_{i}\right)$, em que $h(\cdot)$ é uma função conhecida um-a-um contínua e diferenciável e $\tau_{i}=\mathbf{z}_{i}^{T} \gamma$, em que $\mathbf{z}_{i}=\left(z_{i 1}, \ldots, z_{i q}\right)^{T}$ tem valores de $q$ variáveis explanatórias e $\gamma=\left(\gamma_{1}, \ldots, \gamma_{q}\right)^{T}$. A função $h(\cdot)$ é usualmente chamada de função de ligação de dispersão e deve ser uma função positiva. Uma possível escolha para $h(\cdot)$ é $h(\tau)=\exp (\tau)$. As covariáveis na dispersão, não são necessariamente as mesmas da locação. Quando existem, temos que $\mathrm{E}\left(y_{i}\right)=\mu_{i}$ e $\operatorname{Var}\left(y_{i}\right)=\xi \phi_{i}$, em que $\xi$ foi definido na Seção 1.3. O modelo definido por (3.1)-(3.2) é chamado modelo simétrico linear heteroscedástico.

O logaritmo da função de verossimilhança de $\boldsymbol{\theta}=\left(\boldsymbol{\beta}^{T}, \gamma^{T}\right)^{T}$ fica dado por

$$
\mathrm{L}(\boldsymbol{\theta})=-\frac{1}{2} \sum_{i=1}^{n} \log \left\{\phi_{i}\right\}+\sum_{i=1}^{n} \log \left\{g\left(u_{i}\right)\right\},
$$

em que $u_{i}=\left(y_{i}-\mu_{i}\right)^{2} / \phi_{i}$. As funções escore para $\beta$ e $\gamma$ tomam aqui, respectivamente, as formas

$$
\mathrm{U}_{\beta}(\theta)=\mathrm{X}^{T} \mathrm{D}(\mathrm{g})(\mathrm{y}-\mathrm{X} \boldsymbol{\beta}) \quad \text { e } \quad \mathrm{U}_{\gamma}(\theta)=\mathrm{Z}^{T} \mathrm{~m}
$$


em que $\mathrm{X}$ é uma matriz $n \times p$ com linhas $\mathrm{x}_{i}^{T}, \mathrm{y}=\left(y_{1}, \ldots, y_{n}\right)^{T}, \mathrm{D}(\mathrm{g})=\operatorname{diag}\left\{\mathrm{g}_{1}, \ldots, \mathrm{g}_{n}\right\}$ $\operatorname{com} g_{i}=\frac{v_{i}}{\phi_{i}}, v_{i}=-2 W_{g}\left(u_{i}\right), W_{g}(u)=\frac{g^{\prime}(u)}{g(u)}, g^{\prime}(u)=\frac{\partial g(u)}{\partial u}, \mathbf{m}=\left(m_{1}, \ldots, m_{n}\right)^{T} \operatorname{com}$ $m_{i}=\frac{h_{i}^{\prime}}{2 \phi_{i}}\left(v_{i} u_{i}-1\right)$, em que $h_{i}^{\prime}=\frac{\partial h\left(\tau_{i}\right)}{\partial \tau_{i}}$ e Z é uma matriz $n \times q$ de linhas $\mathbf{z}_{i}^{T}$. Expressões para $W_{g}(u)$ e $W_{g}^{\prime}(u)$ podem ser encontradas na Tabela 2.1.

\subsubsection{Informação de Fisher}

Seja $-\left.\ddot{\mathrm{L}}_{\theta \theta}\right|_{\hat{\theta}}$ a matriz de informação observada de Fisher para $\theta$ dada por

$$
\ddot{\mathrm{L}}_{\theta \theta}=\left[\begin{array}{cc}
\ddot{\mathrm{L}}_{\beta \beta} & \ddot{\mathrm{L}}_{\beta \gamma} \\
\ddot{\mathrm{L}}_{\gamma \beta} & \ddot{\mathrm{L}}_{\gamma \gamma}
\end{array}\right] \text {, }
$$

em que $\ddot{\mathbf{L}}_{\beta \beta}=-\mathrm{X}^{T} \mathrm{D}(\mathrm{a}) \mathrm{X}, \ddot{\mathrm{L}}_{\beta \gamma}=2 \mathrm{X}^{T} \mathrm{D}(\mathrm{b}) \mathrm{Z}$ e $\ddot{\mathrm{L}}_{\gamma \gamma}=-\mathbf{Z}^{T} \mathrm{D}(\mathrm{c}) \mathrm{Z} \operatorname{com} \mathrm{D}(\mathbf{a})=$ $\operatorname{diag}\left\{a_{1}, \ldots, a_{n}\right\}, \mathbf{D}(\mathbf{c})=\operatorname{diag}\left\{c_{1}, \ldots, c_{n}\right\}, \mathbf{b}=\left(b_{1}, \ldots, b_{n}\right)^{T}, \mathbf{u}=\left(u_{1}, \ldots, u_{n}\right)^{T}$, $\mathrm{e}=\left(e_{1}, \ldots, e_{n}\right)^{T}, a_{i}=\frac{1}{\phi_{i}}\left\{v_{i}-4 W_{g}^{\prime}\left(u_{i}\right) u_{i}\right\}, b_{i}=\frac{h_{i}^{\prime}}{\phi_{i}^{2}}\left\{W_{g}\left(u_{i}\right)+u_{i} W_{g}^{\prime}\left(u_{i}\right)\right\} e_{i}, c_{i}=$ $\frac{1}{2 \phi_{i}}\left(h_{i}^{\prime \prime}-\frac{h_{i}^{\prime 2}}{\phi_{i}}\right)-\frac{h_{i}^{\prime 2}}{\phi_{i}^{2}}\left\{W_{g}^{\prime}\left(u_{i}\right) u_{i}^{2}+2 W_{g}\left(u_{i}\right) u_{i}\right\}+\frac{h_{i}^{\prime \prime}}{\phi_{i}} W_{g}\left(u_{i}\right) u_{i}, e_{i}=\left(y_{i}-\mu_{i}\right)$, para $i=$ $1, \ldots, n$.

Depois de algumas manipulações algébricas, encontramos a inversa da matriz de informação observada de Fisher $\left.\ddot{\mathbf{L}}_{\theta \theta}\right|_{\hat{\theta}}$ expressa na forma

$$
\ddot{\mathrm{L}}_{\theta \theta}^{-1}=\left[\begin{array}{cc}
\ddot{\mathrm{L}}_{\beta \beta}^{-1}+\mathrm{AE}^{-1} \mathrm{~A}^{T} & \mathrm{AE}^{-1} \\
\mathrm{E}^{-1} \mathrm{~A}^{T} & \mathrm{E}^{-1}
\end{array}\right],
$$

em que $\mathbf{A}=2\left\{\mathbf{X}^{T} \mathbf{D}(\mathbf{a}) \mathbf{X}\right\}^{-1} \mathbf{X}^{T} \mathbf{D}(\mathbf{b}) \mathbf{Z}$ e $\mathbf{E}=-\mathbf{Z}^{T} \mathbf{D}(\mathbf{c}) \mathbf{Z}+2 \mathbf{Z}^{T} \mathbf{D}(\mathbf{b}) \mathbf{X} \mathbf{A}$.

Podemos mostrar que os parâmetros $\beta$ e $\gamma$ são globalmente ortogonais e a matriz de informação esperada de Fisher $\mathbf{K}_{\theta \theta}$ para $\theta$ é bloco-diagonal, $\mathbf{K}_{\theta \theta}=$ $\operatorname{diag}\left\{\mathbf{K}_{\beta \beta}, \mathbf{K}_{\gamma \gamma}\right\}$. As matrizes de informação esperada de Fisher $\mathbf{K}_{\beta \beta}$ e $\mathbf{K}_{\gamma \gamma}$ para $\beta$ e $\gamma$ são dadas, respectivamente, por $K_{\beta \beta}=\mathrm{X}^{T} \mathbf{W}_{1} \mathrm{X}$ e $\mathbf{K}_{\gamma \gamma}=\mathrm{Z}^{T} \mathrm{~W}_{2} \mathbf{Z}$ em que $\mathbf{W}_{1}=\operatorname{diag}\left\{4 d_{g} / \phi_{i}\right\}$ e $\mathbf{W}_{2}=\operatorname{diag}\left\{\frac{\left(4 f_{g}-1\right) h_{i}^{\prime 2}}{4 \phi_{i}^{2}}\right\}$, para $i=1, \ldots, n$. Um processo iterativo para fornecer as estimativas de máxima verossimilhança de $\beta$ e $\gamma$ pode ser desenvolvido usando, por exemplo, o método scoring de Fisher. As estimativas de máxima verossimilhança $\hat{\boldsymbol{\beta}}$ e $\hat{\gamma}$ são obtidas resolvendo-se o seguinte sistema de equações :

$$
\mathrm{X}^{T} \mathrm{~W}_{1}^{(k)} \mathrm{X} \boldsymbol{\beta}^{(k+1)}=\mathrm{X}^{T} \mathrm{~W}_{1}^{(k)} \mathbf{z}_{\beta}^{(k)} \quad \text { e } \quad \mathrm{Z}^{T} \mathrm{~W}_{2}^{(k)} \mathrm{Z} \gamma^{(k+1)}=\mathrm{Z}^{T} \mathbf{W}_{2}^{(k)} \mathbf{z}_{\gamma}^{(k)}
$$


em que $\mathbf{z}_{\beta}$ e $\mathbf{z}_{\gamma}$ são vetores $n \times 1$ cujas componentes são dadas por

$$
z_{\beta_{i}}=\mu_{i}+\frac{v_{i}}{4 d_{g}}\left(y_{i}-\mu_{i}\right) \quad \text { e } \quad z_{\gamma_{i}}=\tau_{i}+\frac{2 \phi_{i}}{\left(4 f_{g}-1\right) h_{i}^{\prime}}\left(v_{i} u_{i}-1\right),
$$

sendo que $d_{g}=\mathrm{E}\left\{W_{g}^{2}\left(U^{2}\right) U^{2}\right\}$ e $f_{g}=\mathrm{E}\left\{W_{g}^{2}\left(U^{2}\right) U^{4}\right\}$ com $U \sim \mathrm{S}(0,1)$. Valores para $d_{g}$ e $f_{g}$ podem ser encontrados na Tabela 2.2 .

Quando $h(\tau)=\exp (\tau)$, obtemos as expressões simplificadas para $m_{i}=\frac{1}{2}\left(v_{i} u_{i}-\right.$ 1), $b_{i}=\frac{1}{\phi_{i}}\left\{W_{g}\left(u_{i}\right)+u_{i} W_{g}^{\prime}\left(u_{i}\right)\right\} e_{i}, c_{i}=\left\{-W_{g}^{\prime}\left(u_{i}\right) u_{i}-W_{g}\left(u_{i}\right)\right\} u_{i}, W_{2}=\frac{\left(4 f_{g}-1\right)}{4} \mathbf{I}_{n}$ e $z_{\gamma_{i}}=\tau_{i}+\frac{2}{\left(4 f_{g}-1\right)}\left(v_{i} u_{i}-1\right)$. Consequentemente, a matriz de informação esperada de Fisher de $\gamma$ fica dada por $\mathbf{K}_{\gamma \gamma}=\frac{\left(4 f_{g}-1\right)}{4} \mathbf{Z}^{T} \mathbf{Z}$.

Em outro caso, quando $h(\tau)=\tau^{2}$ temos $m_{i}=\frac{1}{\phi^{1 / 2}}\left(v_{i} u_{i}-1\right), b_{i}=\frac{2}{\phi_{i}^{3 / 2}}\left\{W_{g}\left(u_{i}\right)+\right.$ $\left.u_{i} W_{g}^{\prime}\left(u_{i}\right)\right\} e_{i}, c_{i}=-\frac{1}{\phi_{i}}\left\{1+4 W_{g}^{\prime}\left(u_{i}\right) u_{i}^{2}+6 W_{g}\left(u_{i}\right) u_{i}\right\}, W_{2}=\operatorname{diag}\left\{\frac{\left(4 f_{g}-1\right)}{\phi_{i}}\right\}$ e $z_{\gamma_{i}}=$ $\tau_{i}+\frac{\tau_{i}}{\left(4 f_{g}-1\right)}\left(v_{i} u_{i}-1\right)$.

\subsubsection{Testes de heteroscedasticidade}

Assumiremos que $\beta \in \Omega_{\beta} \subset \mathbb{R}^{p}$, em que $\Omega_{\beta}$ é um aberto com pontos interiores. Pode-se mostrar que $\hat{\boldsymbol{\beta}}$ é um estimador consistente de $\boldsymbol{\beta}$, e

$$
\sqrt{n}(\hat{\boldsymbol{\beta}}-\boldsymbol{\beta}) \stackrel{\mathrm{d}}{\rightarrow} \mathrm{N}_{p}\left(0, \mathbf{J}_{\beta \beta}^{-1}\right), \text { em que } \mathbf{J}_{\beta \beta}=\lim _{n \rightarrow \infty} \frac{1}{n} \mathbf{K}_{\beta \beta} .
$$

Então, $\hat{\mathrm{K}}_{\beta \beta}^{-1}=\left(\mathrm{X}^{T} \hat{\mathrm{W}}_{1} \mathrm{X}\right)^{-1}$ é um estimador consistente da matriz de variânciacovariância assintótica de $\hat{\boldsymbol{\beta}}$. Além disso, $\hat{\boldsymbol{\gamma}}$ o estimador de máxima verossimilhança de $\gamma$, é um estimador consistente de $\gamma$, e

$$
\sqrt{n}(\hat{\gamma}-\gamma) \stackrel{\mathrm{d}}{\rightarrow} \mathrm{N}_{q}\left(0, \mathbf{J}_{\gamma \gamma}^{-1}\right), \quad \text { em que } \quad \mathrm{J}_{\gamma \gamma}=\lim _{n \rightarrow \infty} \frac{1}{n} \mathbf{K}_{\gamma \gamma}
$$

Então, $\hat{\mathbf{K}}_{\gamma \gamma}^{-1}=\left(\mathbf{Z}^{T} \hat{\mathbf{W}}_{2} \mathbf{Z}\right)^{-1}$ é um estimador consistente da matriz de variânciacovariância assintótica de $\hat{\gamma}$.

Suponha agora que o interesse aqui é testar a presença de heteroscedasticidade, podemos representá-la pelas hipóteses $\mathrm{H}_{0}: \gamma_{*}=0$ contra $\mathrm{H}_{1}$ : pelo menos $\gamma_{j} \neq$ $0, j=2, \ldots, q$ em que $\gamma_{*}=\left(\gamma_{2}, \ldots, \gamma_{q}\right)^{T}$. Nesse caso $\operatorname{Var}(\hat{\gamma})=\frac{4}{\left(4 f_{g}-1\right)}\left(\mathbf{Z}^{T} \mathbf{W}_{3} \mathbf{Z}\right)^{-1}$ em que $\mathrm{W}_{3}=\operatorname{diag}\left\{w_{3 i}\right\}$ com $w_{3 i}=\frac{h_{i}^{\prime 2}}{4 \phi_{i}^{2}}$ para $i=1, \ldots, n$. Usando alguns resultados 
algébricos obtemos $\operatorname{Var}\left(\hat{\gamma}_{*}\right)=\frac{4}{\left(4 f_{g}-1\right)}\left(\mathbf{Z}_{1}^{T} \mathbf{W}_{3}^{1 / 2} \mathbf{M}_{1} \mathbf{W}_{3}^{1 / 2} \mathbf{Z}_{1}\right)^{-1}$ em que $\mathbf{Z}_{1}$ é dada pela partição $\mathrm{Z}=\left(\mathbf{Z}_{1}, \mathbf{1}_{n}\right)$ sendo $\mathbf{Z}_{1}=\left(\mathbf{z}_{2}^{T}, \ldots, \mathbf{z}_{q}^{T}\right)^{T}$ uma matriz $n \times(q-1), \mathbf{1}_{n}$ um vetor de uns e $\mathrm{M}_{1}=\mathrm{I}-\mathrm{H}_{1}$, em que $\mathrm{H}_{1}=\left(1_{n}^{T} \mathrm{~W}_{3} \mathbf{1}_{n}\right)^{-1} \mathrm{~W}_{3}^{1 / 2} \mathrm{~J}_{n} \mathrm{~W}_{3}^{1 / 2}$ sendo $\mathrm{I}_{n}$ uma matriz identidade de ordem $n$ e $\mathbf{J}_{n}=1_{n} 1_{n}^{T}$. Então,

$$
\begin{aligned}
\operatorname{Var}\left(\hat{\gamma}_{*}\right) & =\frac{4}{\left(4 f_{g}-1\right)}\left(\mathbf{Z}_{1}^{T} \mathbf{W}_{3}^{1 / 2} \mathbf{M}_{1} \mathbf{W}_{3}^{1 / 2} \mathbf{Z}_{1}\right)^{-1} \\
& =\frac{4}{\left(4 f_{g}-1\right)}\left(\mathbf{R}^{T} \mathbf{W}_{3} \mathbf{R}\right)^{-1}
\end{aligned}
$$

em que $\mathbf{R}=\mathbf{Z}_{1}-\mathbf{1}_{n} \mathbf{C}$ e $\mathbf{C}=\left(\mathbf{1}_{n}^{T} \mathbf{W}_{3} \mathbf{1}_{n}\right)^{-1} \mathbf{1}_{n}^{T} \mathbf{W}_{3} \mathbf{Z}_{1}$. Aqui $\mathbf{C}$ é uma matriz $n \times(q-1)$ cuja $j$-ésima coluna é o vetor de coeficientes de regressão linear (com pesos $\mathbf{W}_{3}$ ) da $j$-ésima coluna de $\mathbf{Z}_{1}$ sobre $\mathbf{1}_{n}$. Assim, $\mathbf{R}$ pode ser interpretado como sendo uma matriz $n \times(q-1)$ de resíduos. A $j$-ésima coluna de $\mathbf{R}$ corresponde aos resíduos ordinários da regressão linear (com pesos $\mathbf{W}_{3}$ ) da $j$-ésima coluna de $\mathbf{Z}_{1}$ sobre $\mathbf{1}_{n}$. Assim, as estatísticas para os testes da razão de verossimilhanças, Wald e escore são dadas aqui, respectivamente, por

$$
\begin{aligned}
\xi_{R V}^{h} & =2\left\{\mathrm{~L}\left(\hat{\boldsymbol{\beta}}, \hat{\boldsymbol{\gamma}}_{*}, \hat{\gamma}_{1}\right)-\mathrm{L}\left(\hat{\boldsymbol{\beta}}^{0}, \hat{\gamma}_{1}^{0}\right)\right\} \\
\xi_{W}^{h} & =\frac{\left(4 f_{g}-1\right)}{4} \hat{\boldsymbol{\gamma}}_{*}^{T} \hat{\mathbf{R}}^{T} \hat{\mathbf{W}}_{3} \hat{\mathbf{R}} \hat{\boldsymbol{\gamma}}_{*} \mathrm{e} \\
\xi_{S R}^{h} & =\frac{4}{\left(4 f_{g}-1\right)} \hat{\mathbf{m}}_{0}^{T} \mathbf{Z}_{1}\left(\hat{\mathbf{R}}_{0}^{T} \hat{\mathbf{W}}_{3}^{0} \hat{\mathbf{R}}_{0}\right)^{-1} \mathbf{Z}_{1}^{T} \hat{\mathbf{m}}_{0} \\
& =\frac{1}{\left(4 f_{g}-1\right)}\left(\hat{\mathbf{r}}_{e}^{0}\right)^{T} \mathbf{Z}_{1}\left\{\mathbf{Z}_{1}^{T}\left(\mathbf{I}_{n}-\frac{1}{n} \mathbf{J}_{n}\right) \mathbf{Z}_{1}\right\}^{-1} \mathbf{Z}_{1}^{T} \hat{\mathbf{r}}_{e}
\end{aligned}
$$

em que $\hat{\mathbf{m}}_{0}, \mathbf{r}_{e}^{0}$ são vetores avaliados sob $\mathrm{H}_{0}$, com $\mathbf{r}_{e}=\left(v_{1} u_{1}-1, \ldots, v_{n} u_{n}-1\right)^{T}$, isto é, sob o modelo homoscedástico. Segue-se que sob $\mathrm{H}_{0}$ e para $n$ suficientemente grande temos que $\xi_{R V}^{h}, \xi_{W}^{h}$ e $\xi_{S R}^{h}$ têm distribuição qui-quadrado com $(q-1)$ graus de liberdade. Nesse caso, o teste escore é muito atraente pois exige somente o ajuste do modelo homoscedástico.

No caso em que temos a função de ligação de dispersão $h\left(\tau_{i}\right)=\exp \left(\gamma_{1}+\right.$ 
$\left.\sum_{j=2}^{q} \gamma_{j} z_{j i}\right)$ temos as matrizes $\mathbf{M}_{1}=\mathrm{I}_{n}-\frac{1}{n} \mathbf{J}_{n}$ e $\mathrm{W}_{3}=\mathrm{I}_{n}$, então

$$
\begin{aligned}
\operatorname{Var}\left(\hat{\gamma}_{*}\right) & =\frac{4}{\left(4 f_{g}-1\right)}\left\{\mathbf{Z}_{1}^{T}\left(\mathbf{I}_{n}-\frac{1}{n} \mathbf{J}_{n}\right) \mathbf{Z}_{1}\right\}^{-1} \\
& =\frac{4}{\left(4 f_{g}-1\right)}\left(\mathbf{R}^{T} \mathbf{R}\right)^{-1},
\end{aligned}
$$

em que $\mathbf{R}=\mathbf{Z}_{1}-\mathbf{1}_{n} \overline{\mathbf{Z}}_{1}, \overline{\mathbf{Z}}_{1}=\left(\overline{\mathbf{Z}}_{2}, \ldots, \overline{\mathbf{z}}_{q}\right)$ e $\overline{\mathbf{z}}_{j}$ é a média da $j$-ésima coluna $\mathbf{Z}_{1}$. As estatísticas para os testes da razão de verossimilhanças, Wald e escore são dadas aqui, respectivamente, por

$$
\begin{aligned}
\xi_{R V}^{h} & =2\left\{\mathrm{~L}\left(\hat{\boldsymbol{\beta}}, \hat{\boldsymbol{\gamma}}_{*} \hat{\gamma}_{1}\right)-\mathrm{L}\left(\hat{\boldsymbol{\beta}}^{0}, \hat{\gamma}_{1}^{0}\right)\right\} \\
\xi_{W}^{h} & =\frac{\left(4 f_{g}-1\right)}{4} \hat{\gamma}_{*}^{T}\left\{\mathbf{Z}_{1}^{T}\left(\mathbf{I}_{n}-\frac{1}{n} \mathbf{J}_{n}\right) \mathbf{Z}_{1}\right\} \hat{\boldsymbol{\gamma}}_{*} \\
& =\frac{\left(4 f_{g}-1\right)}{4} \hat{\boldsymbol{\gamma}}_{*}^{T} \mathbf{R}^{T} \mathbf{R} \hat{\boldsymbol{\gamma}}_{*} \mathrm{e} \\
\xi_{S R}^{h} & =\frac{4}{\left(4 f_{g}-1\right)} \hat{\mathbf{m}}_{0}^{T} \mathbf{Z}_{1}\left(\mathbf{R}^{T} \mathbf{R}\right)^{-1} \mathbf{Z}_{1}^{T} \hat{\mathbf{m}}_{0} \\
& =\frac{4}{\left(4 f_{g}-1\right)} \hat{\mathbf{m}}_{0}^{T} \mathbf{Z}_{1}\left\{\mathbf{Z}_{1}^{T}\left(\mathbf{I}_{n}-\frac{1}{n} \mathbf{J}_{n}\right) \mathbf{Z}_{1}\right\}^{-1} \mathbf{Z}_{1}^{T} \hat{\mathbf{m}}_{0} .
\end{aligned}
$$

\subsection{Resíduos}

De maneira análoga à Seção 2.3, consideraremos aqui o resíduo ordinário com $\phi_{i}$ conhecido ou fixo expresso na seguinte forma :

$$
r_{i}\left(y_{i}, \hat{\boldsymbol{\beta}}, \phi_{i}\right)=y_{i}-\hat{y}_{i}, \quad i=1, \ldots, n
$$

e procedendo como na Seção 2.3 tem-se até ordem $n^{-1}$ que

$$
\mathrm{E}\left(r_{i}\right)=\mathrm{E}\left(\epsilon_{i}\right)+\mathrm{E}\left(\hat{\beta}_{r}-\beta_{r}\right) \mathrm{E}\left(H_{r}^{i}\right)+I^{r s} \mathrm{E}\left(H_{r}^{i} U_{s}^{i}+\frac{1}{2} H_{r s}^{i}\right)
$$

e

$$
\mathrm{E}\left(r_{i}^{2}\right)=\mathrm{E}\left(\epsilon_{i}^{2}\right)+2 \mathrm{E}\left(\hat{\beta}_{r}-\beta_{r}\right) \mathrm{E}\left(\epsilon_{i} H_{r}^{i}\right)+2 I^{r s} \mathrm{E}\left(\epsilon_{i} H_{r}^{i} U_{s}^{i}+\frac{1}{2} H_{r}^{i} H_{s}^{i}+\frac{1}{2} \epsilon_{i} H_{r s}^{i}\right),
$$

sendo que no lado direito da equação a convenção da soma é aplicada em $r, s=$ $1, \ldots, p, \epsilon_{i}=h\left(y_{i}, \boldsymbol{\beta}\right)=y_{i}-\mu_{i}, \mathrm{E}\left(\hat{\beta}_{r}-\beta_{r}\right)$ é o viés de $\beta_{r}$ de ordem $n^{-1}$, que nesse 
caso é nulo, $H_{r}^{i}$ e $H_{r s}^{i}$ denotam, respectivamente, a primeira e a segunda derivadas de $h\left(y_{i}, \beta\right)$ com respeito a $\beta_{r}$ e $\left(\beta_{r}, \beta_{s}\right), U_{s}^{i}$ é a função escore $\partial \mathrm{L}\left(\boldsymbol{\theta} ; y_{i}\right) / \partial \beta_{s}$ e $I^{r s}$ denota o $(r, s)$-ésimo elemento da matriz $\mathbf{K}_{\beta \beta}^{-1}$.

Então, encontramos $\mathrm{E}\left(\epsilon_{i}\right)=0, \mathrm{E}\left(H_{r}^{i}\right)=-x_{i r}, \mathrm{E}\left(H_{r s}^{i}\right)=0$ e $\mathrm{E}\left(H_{r}^{i} U_{s}^{i}\right)=0$ de modo que $\mathrm{E}\left(r_{i}\right)=0$.

Além disso, obtemos que $\mathrm{E}\left(\epsilon_{i}^{2}\right)=\xi \phi_{i}, \mathrm{E}\left(\epsilon_{i} H_{r}^{i}\right)=0, \mathrm{E}\left(\epsilon_{i} H_{r}^{i} U_{s}^{i}\right)=x_{i r} x_{i s}, \mathrm{E}\left(H_{r}^{i} H_{s}^{i}\right)=$ $x_{i r} x_{i s}$ e $\mathrm{E}\left(\epsilon_{i} H_{r s}^{i}\right)=0$. Assim, até ordem $n^{-1}$

$$
\begin{aligned}
\mathrm{E}\left(r_{i}^{2}\right) & =\xi \phi_{i}-I^{r s} x_{i r} x_{i s} \mathrm{e} \\
\operatorname{Var}\left(r_{i}\right) & =\xi \phi_{i}\left\{1-\left(4 d_{g} \xi\right)^{-1} a_{r s} x_{i r} x_{i s}\right\} \\
& =\xi \phi_{i}\left\{1-\left(4 d_{g} \xi\right)^{-1} h_{i i}\right\}
\end{aligned}
$$

em que $a_{r s}$ é o $(r, s)$-elemento da matriz $\left(\mathrm{X}^{T} \boldsymbol{\Phi}^{-1} \mathrm{X}\right)^{-1}, \Phi=\operatorname{diag}\left\{\phi_{1}, \ldots, \phi_{n}\right\}$, $h_{i j}=\phi_{i}^{-1 / 2} \mathrm{x}_{i}^{T}\left(\mathrm{X}^{T} \Phi^{-1} \mathrm{X}\right)^{-1} \mathrm{x}_{j} \phi_{j}^{-1 / 2}$ e $\Phi^{-1 / 2}=\operatorname{diag}\left\{\phi_{1}^{-1 / 2}, \ldots, \phi_{n}^{-1 / 2}\right\}$. Ainda, obtemos até ordem $n^{-1}, \mathrm{E}\left(r_{i} r_{j}\right)=-I^{r s} x_{j r} x_{i s}$. Portanto, em forma matricial

$$
\operatorname{Var}(\mathrm{r})=\xi \Phi\left\{\mathrm{I}_{n}-\left(4 d_{g} \xi\right)^{-1} \mathbf{H}\right\}
$$

em que $\mathrm{H}=\boldsymbol{\Phi}^{-1 / 2} \mathbf{X}\left(\mathrm{X}^{T} \boldsymbol{\Phi}^{-1} \mathbf{X}\right)^{-1} \mathbf{X}^{T} \boldsymbol{\Phi}^{-1 / 2}$ e $\mathbf{I}_{n}$ é a matriz identidade de ordem $n$. Sendo assim, uma forma natural de definir um resíduo padronizado é dada por

$$
\begin{aligned}
r_{t_{i}} & =\frac{r_{i}}{\left\{\xi \hat{\phi}_{i}\right\}^{1 / 2}\left(1-\left(4 d_{g} \xi\right)^{-1} \hat{h}_{i i}\right)^{1 / 2}} \\
& =\frac{y_{i}-\hat{y}_{i}}{\left\{\xi \hat{\phi}_{i}\right\}^{1 / 2}\left\{1-\left(4 d_{g} \xi\right)^{-1} \hat{h}_{i i}\right\}^{1 / 2}}, \quad i=1, \ldots, n .
\end{aligned}
$$

Estudos de simulação têm mostrado que o resíduo proposto $r_{t_{i}}$ tem média e variância aproximadamente zero e um, respectivamente, uma assimetria desprezível e uma curtose acompanhando a curtose da distribuição (veja, por exemplo, Tabela $3.1)$. 
Tabela 3.1 Análise descritiva para o resíduo $r_{t_{i}}$ de 1000 observações geradas para o modelo ajustado na Seção 3.4.

\begin{tabular}{c|ccc}
\hline Estatística & Normal & $t_{4}$ & Logístico-II \\
\hline média & $-0,003$ & 0,006 & $-0,003$ \\
variância & 0,999 & 0,932 & 0,952 \\
assimetria & 0,005 & $-0,044$ & 0,008 \\
curtose & $-0,326$ & 1,583 & 0,476 \\
\hline
\end{tabular}

\subsection{Aplicação}

Como ilustração usaremos o conjunto de dados discutidos em Montgomery, Peck e Vining (2001, Tabela 3.2), apresentado no Apêndice D, cujo interesse principal é predizer quanto tempo é requerido pelo motorista da rota para o serviço de manutenção e reposição de latas e garrafas de bebidas em máquinas de vendas. A atividade de serviço inclui estocagem em máquinas com produtos e sua manutenção. Ajustou-se um modelo linear supondo variância constante, cuja variável resposta é o tempo gasto no serviço, y (em minutos), e as covariadas são o número de bebidas estocadas $\left(x_{1}\right)$ e a distância percorrida pelo motorista ( $x_{2}$ em pés) numa amostra de 25 observações. Na análise de diagnóstico, os pontos 9 e 22 aparecem com uma grande influência nas estimativas dos três parâmetros (veja Montgomery, Peck e Vining, 2001, pp. 210,213,215,216,217). Ferrari, Cysneiros e Cribari-Neto (2004) ajustaram o modelo de regressão linear para esse conjunto de dados excluindo os casos 9 e 22 , dado por $y_{i}=\beta_{0}+\beta_{1} x_{i 1}+\beta_{1} x_{i 2}+\epsilon_{i}, i=1, \ldots, 23$, em que $\epsilon_{i} \sim N\left(0, \sigma^{2} \exp \left\{\delta_{1} x_{i 1}+\delta_{2} x_{i 2}\right\}\right)$ sendo detectada a presença de heteroscedasticidade, baseada no teste da razão de verossimilhanças.

Propomos ajustar o modelo heteroscedástico para o conjunto de dados completos sob erros com distribuições com caudas mais pesadas do que a normal, com a finalidade de tentar acomodar esses dois pontos aberrantes. O modelo é dado por

$$
y_{i}=\beta_{0}+\beta_{1} x_{i 1}+\beta_{1} x_{i 2}+\sqrt{\phi_{i}} \epsilon_{i}, \quad i=1, \ldots, 25
$$


em que $\phi_{i}=\exp \left\{\alpha+\delta_{1}\left(x_{i 1}-\overline{x_{1}}\right)+\delta_{2}\left(x_{i 2}-\overline{x_{2}}\right)\right\}=\sigma^{2} \exp \left\{\delta_{1}\left(x_{i 1}-\overline{x_{1}}\right)+\delta_{2}\left(x_{i 2}-\overline{x_{2}}\right)\right\}$, $\epsilon_{i} \sim \mathrm{S}(0,1)$ são erros mutuamente independentes.

Tentamos várias distribuições para o erro, porém somente dois modelos parecem ajustar aos dados tão bem quanto, ou melhor do que o modelo normal: o modelo $t$-Student com 4 graus de liberdade e o modelo logístico-II. Para o modelo ajustado a variável $x_{1}$ foi removida da parte sistemática referente à dispersão. Lange, Litte e Taylor (1989) sugerem que os graus de liberdade devem ser fixados para amostras pequenas. Mencionam que $\nu=4$ tem funcionado bem para algumas aplicações.

As estimativas de máxima verossimilhança dos três modelos são apresentadas na Tabela 3.2. As estatísticas descritas na Seção 3.2 .2 para avaliar $\mathrm{H}_{0}: \delta_{2}=0$ contra $\mathrm{H}_{0}: \delta_{2} \neq 0$ foram altamente significativas para os três modelos (veja Tabela 3.3). Os gráficos normais de probabilidades com envelope para os três modelos postulados não apresentam nenhum comportamento não usual. Contudo, os modelos logístico-II e $t$-Student parecem acomodar melhor os pontos. Além disso, o gráfico de resíduos contra os valores ajustados sob erro normal indica que os pontos 4 e 21 têm resíduo alto (veja Figura 3.1). Entretanto, o gráfico de resíduos contra os valores ajustados sob erro $t$-Student e logístico-II indicam apenas a presença da observação 4 (veja as Figuras 3.2 - 3.3) como aberrante. No capítulo 4 continuaremos a análise desse exemplo para o qual, baseado em métodos de validação, escolheremos dentre esses modelos o que melhor se adequa aos dados. 
Tabela 3.2 Estimativas de máxima verossimilhança (erro padrão aproximado) para alguns modelos simétricos ajustados aos dados de estoque da Tabela D.1.

\begin{tabular}{c|ccc}
\hline Parâmetro & Normal & $t_{4}$ & Logístico-II \\
\hline$\beta_{0}$ & 4,682 & 4,321 & 4,432 \\
& $(0,815)$ & $(0,752)$ & $(0,789)$ \\
$\beta_{1}$ & 1,459 & 1,437 & 1,450 \\
& $(0,143)$ & $(0,133)$ & $(0,139)$ \\
$\beta_{2}$ & 0,011 & 0,012 & 0,011 \\
& $(0,003)$ & $(0,002)$ & $(0,003)$ \\
$\alpha$ & 1,745 & 1,268 & 0,586 \\
& $(0,283)$ & $(0,374)$ & $(0,334)$ \\
$\delta_{2}$ & 0,003 & 0,003 & 0,003 \\
& $(0,001)$ & $(0,001)$ & $(0,001)$ \\
\hline
\end{tabular}

Tabela 3.3 Valor das estatísticas dos testes e p-valor (entre parênteses) para alguns modelos simétricos ajustados aos dados de estoque da Tabela D.1.

\begin{tabular}{c|ccc}
\hline Estatística & Normal & $t_{4}$ & Logístico-II \\
\hline$\xi_{S R}^{h}$ & 11,722 & 10,772 & 15,079 \\
& $(0,0006)$ & $(0,0010)$ & $(0,0001)$ \\
$\xi_{R V}^{h}$ & 12,511 & 9,843 & 11,374 \\
& $(0,0004)$ & $(0,0017)$ & $(0,0007)$ \\
$\xi_{W}^{h}$ & 10,000 & 8,084 & 8,532 \\
& $(0,0016)$ & $(0,0045)$ & $(0,0035)$ \\
\hline
\end{tabular}


Figura 3.1 Gráfico normal de probabilidades com envelope para $r_{t_{i}}$ (esquerda) $e$ gráfico de resíduos $r_{t_{i}}$ contra os valores ajustados para o modelo normal (direita), referente ao modelo ajustado aos dados de estoque da Tabela D.1.
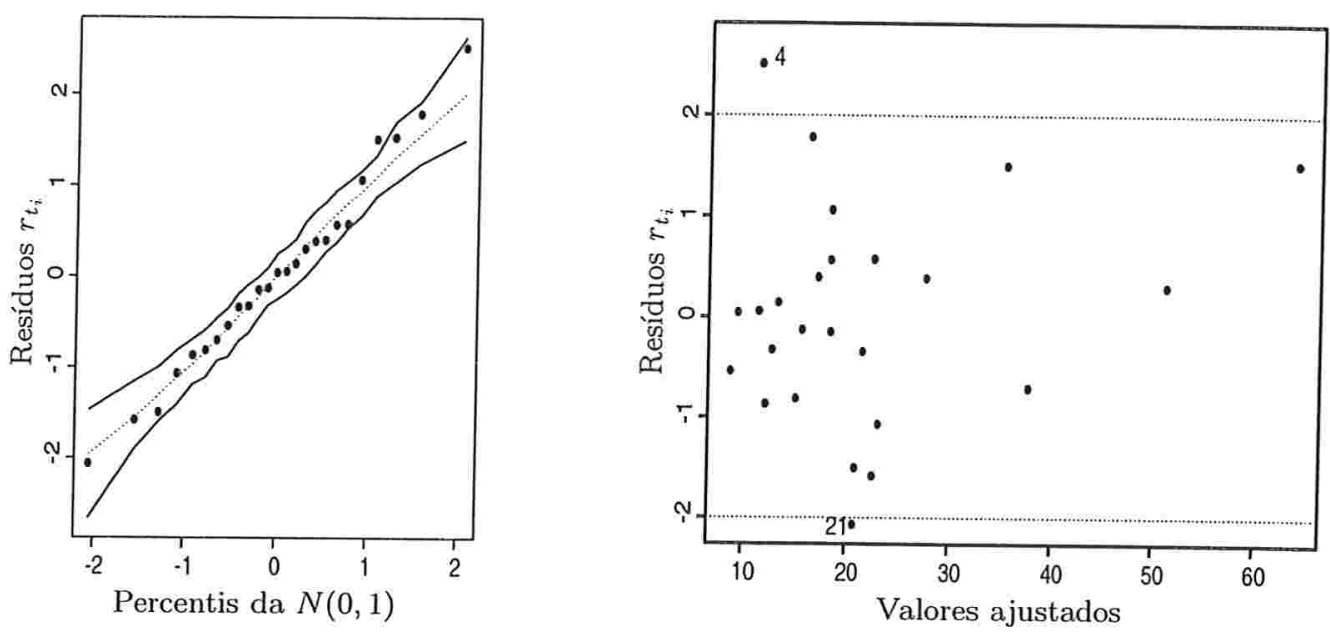

Figura 3.2 Gráfico normal de probabilidades com envelope para $r_{t_{i}}$ (esquerda) $e$ gráfico de resíduos $r_{t_{i}}$ contra os valores ajustados para o modelo $t$-Student com 4 g.l. (direita), referente ao modelo ajustado aos dados de estoque da Tabela D.1.
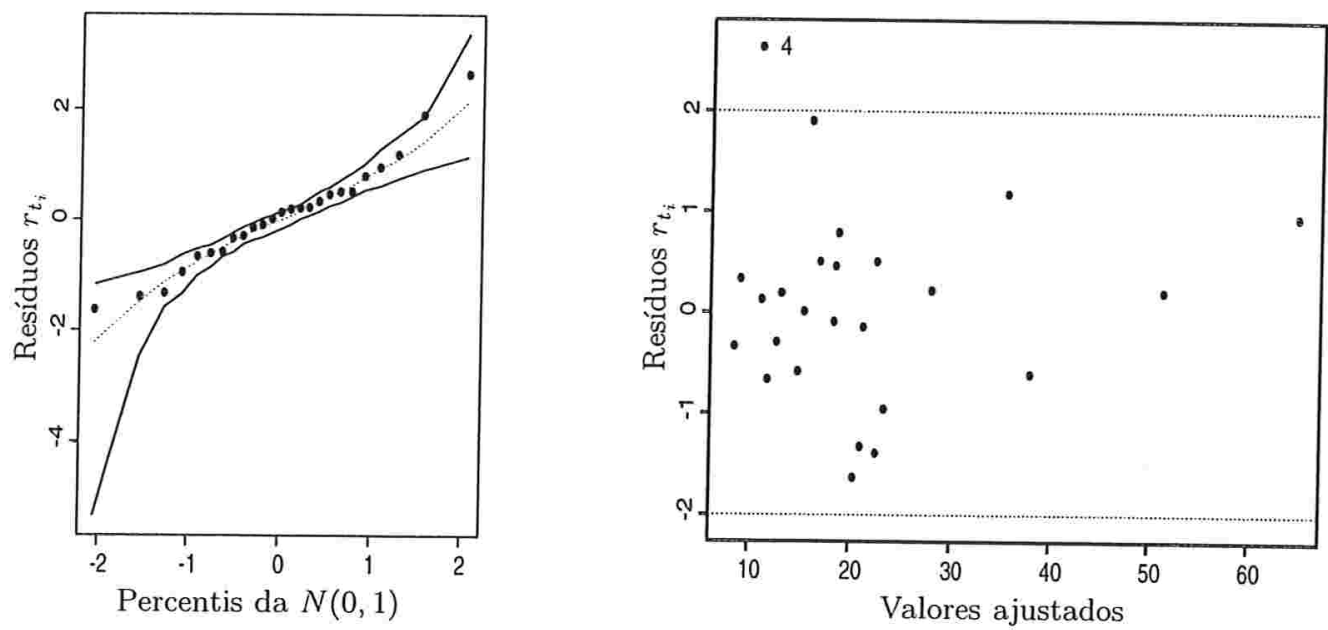
Figura 3.3 Gráfico normal de probabilidades com envelope para $r_{t_{i}}$ (esquerda) e gráfico de resíduos $r_{t_{i}}$ contra os valores ajustados para o modelo logístico-II (direita), referente ao modelo ajustados aos dados de estoque da Tabela D.1.
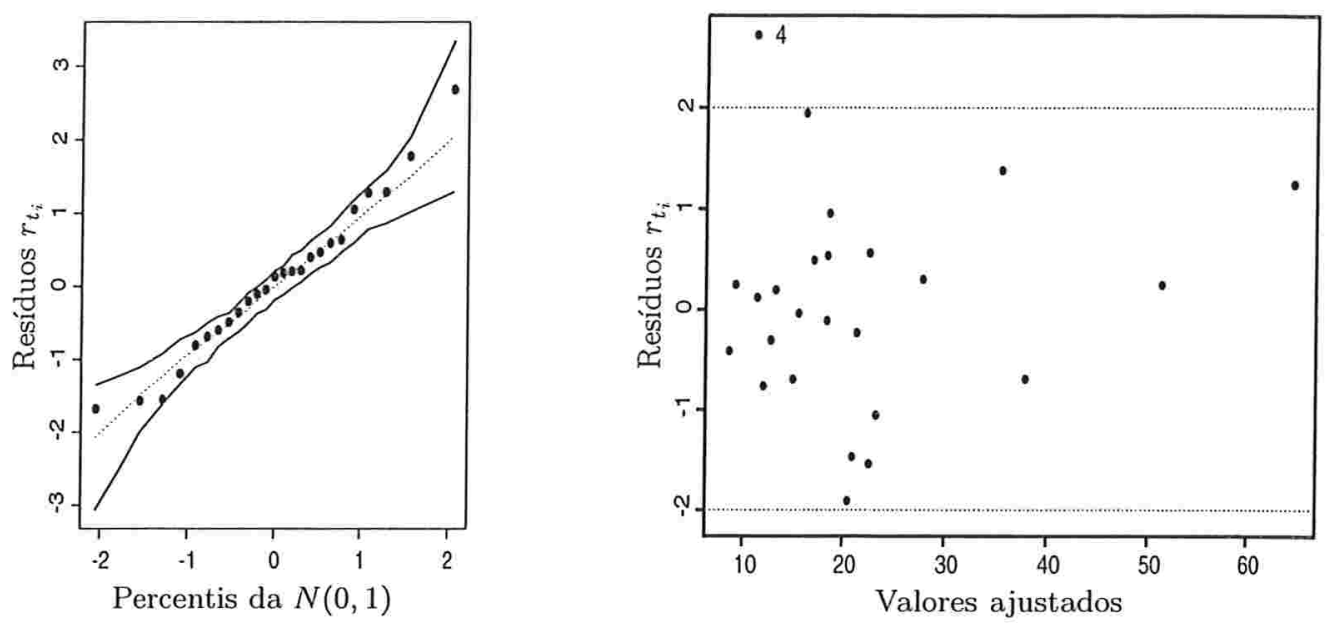


\section{CAPÍTULO 4}

\section{Diagnósticos em modelos simétricos}

\subsection{Influência local no afastamento da verossimilhança}

A idéia principal de influência local é verificar, através de alguma medida apropriada de influência, o efeito de pequenas perturbações no modelo ou nos dados. Se essas perturbações causarem efeitos desproporcionais em determinados resultados do modelo, podem ser indícios de que o modelo está mal ajustado ou que existem afastamentos importantes das suposições feitas para o mesmo. A identificação das observações responsáveis por essas discrepâncias pode ajudar na escolha de um modelo mais adequado aos dados. A medida de influência mais conhecida é o afastamento da verossimilhança $\mathrm{LD}(\boldsymbol{\omega})=2\left\{\mathrm{~L}(\hat{\boldsymbol{\theta}})-\mathrm{L}\left(\hat{\boldsymbol{\theta}}_{\omega}\right)\right\}$, em que $\hat{\boldsymbol{\theta}}_{\boldsymbol{\omega}}$ denota a estimativa de máxima verossimilhança sob o modelo perturbado e $\omega=\left(\omega_{1}, \ldots, \omega_{s}\right)^{T}$ é o vetor de perturbações aplicadas no modelo. A proposta de Cook (1986) é estudar o comportamento de $\operatorname{LD}(\boldsymbol{\omega})$, ou de alguma outra medida de influência, em torno do vetor de não-perturbação $\omega_{0}$. Tem-se que $\operatorname{LD}\left(\omega_{0}\right)=0$. Logo, desde que $\mathrm{LD}(\boldsymbol{\omega}) \geqslant 0, \boldsymbol{\omega}_{0}$ é um ponto de mínimo da função $\mathrm{LD}(\boldsymbol{\omega})$. A sugestão de Cook (1986) é investigar a curvatura normal da linha projetada $\operatorname{LD}\left(\omega_{0}+a \ell\right)$, em que $a \in \mathbb{R}$, em torno de $a=0$ para alguma direção arbitrária $\ell,\|\ell\|=1$. Mostra-se que a curvatura normal pode ser expressa numa forma geral $\mathrm{C}_{\ell}(\theta)=2\left|\ell^{T} \Delta^{T} \ddot{\mathrm{L}}_{\theta \theta}^{-1} \Delta \ell\right|$, em que $\Delta$ é uma matriz $(p+q) \times s$ com elementos $\Delta_{i j}=\partial^{2} L(\theta \mid \omega) / \partial \theta_{i} \partial \omega_{j}$, $i=1, \ldots, p+q$ e $j=1, \ldots, s$, com todas as quantidades sendo avaliadas em $\omega=\omega_{0}$ e $\theta=\hat{\theta}$. Cook sugere tomar a direção correspondente à maior curvatura, denotada por $\ell_{\max }$, o maior autovetor e a correspondente $\mathrm{C}_{\boldsymbol{\ell}_{\max }}$, o maior autovalor da matriz $\mathrm{B}=-\Delta^{T} \ddot{\mathrm{L}}_{\theta \theta}^{-1} \Delta$. O gráfico de índices de $\ell_{\max }$ pode mostrar como se deve perturbar, por exemplo, o parâmetro de escala para obter maiores mudanças nas estimativas de $\boldsymbol{\theta}$. Contudo, se o interesse é somente no vetor $\boldsymbol{\beta}$, a curvatura 
normal na direção $\ell$ é dada por $\mathrm{C}_{\ell}(\boldsymbol{\beta})=2\left|\ell^{T} \Delta^{T}\left(\ddot{\mathrm{L}}_{\theta \theta}^{-1}-\mathrm{L}_{1}\right) \Delta \ell\right|($ veja Cook, 1986), em que

$$
\mathrm{L}_{1}=\left[\begin{array}{cc}
0 & 0 \\
0 & \ddot{\mathbf{L}}_{\gamma \gamma}^{-1}
\end{array}\right]
$$

com $-\left.\ddot{\mathbf{L}}_{\gamma \gamma}\right|_{\hat{\theta}}$ sendo a matriz de informação observada de Fisher para $\gamma$. O gráfico de índices do maior autovetor de $\Delta^{T}\left(\ddot{\mathrm{L}}_{\theta \theta}^{-1}-\mathrm{L}_{1}\right) \Delta$ pode revelar quais observações são influentes em $\hat{\beta}$. Similarmente, a curvatura normal para o parâmetro de escala $\gamma$ na direção $\ell$ é dada por $C_{\ell}(\gamma)=2\left|\ell^{T} \Delta^{T}\left(\ddot{\mathbf{L}}_{\theta \theta}^{-1}-\mathrm{L}_{2}\right) \Delta \ell\right|$, em que

$$
\mathrm{L}_{2}=\left[\begin{array}{cc}
\ddot{\mathrm{L}}_{\beta \beta}^{-1} & 0 \\
0 & 0
\end{array}\right] \text {, }
$$

com $-\left.\ddot{\mathbf{L}}_{\beta \beta}\right|_{\hat{\theta}}$ sendo a matriz de informação observada de Fisher para $\beta$. A influência local das observações em $\hat{\gamma}$ pode ser avaliada considerando-se o gráfico de índices de $\ell_{\text {max }}$ para a matriz $\left|\Delta^{T}\left(\ddot{\mathbf{L}}_{\theta \theta}^{-1}-\mathbf{L}_{2}\right) \Delta\right|$.

Escobar e Meeker (1992) sugerem tomar como medida de influência os elementos da diagonal principal da matriz $\mathrm{B}=-\Delta^{T} \ddot{\mathrm{L}}_{\theta \theta}^{-1} \Delta$, enquanto Lesaffre and Verbeke (1998) sugerem avaliar a curvatura normal na direção da $i$-ésima observação, que consiste na avaliação de $\mathrm{C}_{\ell}(\theta)$ no vetor $(n \times 1) \ell_{i}$ formado por zeros com um na $i$-ésima posição. Essa curvatura é denominada por $\mathrm{C}_{i}$ que é igual a $2\left|b_{i i}\right|$. É sugerido que as observações tais que $\mathrm{C}_{i}>2 \overline{\mathrm{C}}$ tenham uma atenção especial.

Em particular, fazendo uma perturbação aditiva no $i$-ésimo valor da resposta, $y_{i \omega}=y_{i}+\sigma \omega_{i}$ em que $\omega_{i} \in \mathbb{R}$, e $\sigma$ é o desvio padrão de $y_{i}$, podemos considerar a mudança instantânea no $i$-ésimo valor predito (quando $\omega_{i} \rightarrow 0$ ) como uma medida de influência da $i$-ésima observação no seu próprio valor predito. Podemos citar outros esquemas de perturbação de interesse, como por exemplo :

- supor que se deseja verificar a possibilidade das respostas possuírem variâncias distintas, isto é, $\operatorname{Var}\left(y_{i}\right)=\xi \phi / \omega_{i}$, ou seja, a possibilidade de termos um modelo heteroscedástico;

- interesse em perturbar a $t$-ésima variável explicativa, com $\left(x_{i 1}, \ldots, x_{i t}+s_{t} \omega_{i}, \ldots x_{i p}\right)$, em que $s_{t}$ é um fator de escala, que pode ser a norma da $t$-ésima coluna da matriz $\mathrm{X}$. 
É possível perturbar o modelo proposto de diversas outras maneiras, porém é importante escolher esquemas de perturbação e medidas de influência que permitam interpretações fácies. Galea, Bolfarine e Vilca-Labra (2002) estudaram influência local nos modelos com erros nas variáveis sob a distribuição $t$-Student. Galea, Paula e Bolfarine (1997) e Galea, Paula e Uribe-Opazo (2003) investigaram a influência das observações nas estimativas dos parâmetros usando o enfoque de influência local na classe dos modelos simétricos lineares.

\subsubsection{Perturbação na escala no modelo simétrico não-linear}

Considere agora o modelo heteroscedástico

$$
f_{y_{i}}\left(y_{i} \mid \omega_{i}\right)=\sqrt{\frac{\omega_{i}}{\phi}} g\left(\omega_{i} u_{i}\right)
$$

em que $\omega_{i}$ denota o peso correspondente ao $i$-ésimo caso, $i=1, \ldots, n$. Quando $\omega_{i}=1$, o modelo perturbado (4.1) reduz ao modelo postulado (2.2). Além disso, estamos perturbando o parâmetro de escala pela mudança do seu valor para $\phi / \omega_{i}$ para a $i$-ésima observação. A matriz $(p+1) \times n \Delta$ fica nesse caso dada por

$$
\Delta=\left(\begin{array}{c}
-\frac{2}{\phi} \mathrm{D}_{\beta} \mathrm{D}(\mathrm{b}) \\
-\frac{1}{\phi^{2}} \mathrm{e}^{T} \mathrm{D}(\mathrm{b})
\end{array}\right),
$$

em que $b_{i}=\left\{W_{g}\left(u_{i}\right)+u_{i} W_{g}^{\prime}\left(u_{i}\right)\right\} e_{i}, \mathbf{D}(\mathbf{b})=\operatorname{diag}\left(b_{1}, \ldots, b_{n}\right)$ e $e_{i}=y_{i}-\mu_{i}$, para $i=1, \ldots, n$.

\subsubsection{Perturbação de casos no modelo simétrico linear heterocedástico}

Considere o logaritmo da função de verossimilhança de $\theta$ expresso na forma

$$
\mathbf{L}\left(\theta \mid \omega_{i}\right)=\sum_{i=1}^{n} \omega_{i} \log \left\{\frac{u_{i}}{\sqrt{\phi}_{i}}\right\},
$$

em que $0 \leqslant \omega_{i} \leqslant 1$. Sob esse esquema de perturbação a matriz $\Delta$ assume a forma

$$
\Delta=\left(\begin{array}{l}
\mathrm{D}(\mathrm{g}) \mathrm{D}(\mathrm{e}) \mathrm{X} \\
\mathrm{D}(\mathrm{m}) \mathrm{Z}
\end{array}\right),
$$

em que $e_{i}=y_{i}-\mu_{i}$, para $i=1, \ldots, n, \mathbf{D}(\mathrm{g})=\operatorname{diag}\left\{\mathrm{g}_{1}, \ldots, \mathrm{g}_{n}\right\}, \mathbf{D}(\mathbf{e})=\operatorname{diag}\left\{e_{1}, \ldots, e_{n}\right\}$ e $\mathbf{D}(\mathbf{m})=\operatorname{diag}\left\{m_{1}, \ldots, m_{n}\right\}$ estão definidos na Seção 3.2.1. 


\subsection{Influência local na predição}

Seja q um vetor $p \times 1$ de valores das variáveis explanatórias, para o qual não temos necessariamente uma resposta observada. Então, a predição em q é dada por $\hat{\mu}(\mathbf{q})=\sum_{j=1}^{p} q_{j} \hat{\beta}_{j}$. Analogamente, o ponto predito em q baseado no modelo perturbado é dado por $\hat{\mu}(\mathbf{q}, \omega)=\sum_{j=1}^{p} q_{j} \hat{\beta}_{j w}$, em que $\hat{\beta}_{\omega}=\left(\hat{\beta}_{1 \omega}, \ldots, \hat{\beta}_{p \omega}\right)^{T}$ denota a estimativa de máxima verossimilhança do modelo perturbado. Thomas e Cook (1990) têm investigado o efeito de pequenas perturbações na predição em algum particular ponto q em modelos lineares generalizados contínuos assumindo $\phi$ conhecido ou estimado separadamente de $\hat{\beta}$. Contudo, como não é tão claro definir o afastamento da verossimilhança para predições para as quais não se tem nenhuma resposta observada, três funções objetivo baseadas em diferentes resíduos foram definidas. A função objetivo $f(\mathbf{q}, \boldsymbol{\omega})=\{\hat{\mu}(\mathbf{q})-\hat{\mu}(\mathbf{q}, \boldsymbol{\omega})\}^{2}$ tem sido escolhida devido à simplicidade e invariância com respeito a outras medidas de influência.

Similarmente, concentraremos nossos estudos na investigação da curvatura normal na superfície formada pelo vetor $\omega$ e a função $f(q, \omega)$ em torno de $\boldsymbol{\omega}=\boldsymbol{\omega}_{0}$, em que $\boldsymbol{\omega}_{0}$ é tal que $\hat{\boldsymbol{\beta}}_{\omega_{0}}=\hat{\boldsymbol{\beta}}$. A curvatura normal na direção unitária $\ell$ toma, nesse caso, a forma $C_{\ell}=\left|\ell^{T} \ddot{\mathrm{f}} \ell\right|$, em que $\ddot{\mathrm{f}}=\partial^{2} f / \partial \omega \partial \omega^{T}$ é avaliada em $\omega_{0}$ e $\hat{\boldsymbol{\beta}}$. Seguindo Thomas e Cook (1990), obtemos

$$
\ddot{\mathbf{f}}=-2 \Delta^{T}\left(\ddot{\mathbf{L}}_{\beta \beta}^{-1} \mathbf{q q}^{T} \mathrm{~L}_{\beta \beta}^{-1}\right) \Delta
$$

em que $\Delta=\partial^{2} \mathrm{~L}(\boldsymbol{\theta} \mid \boldsymbol{\omega}) / \partial \beta \partial \boldsymbol{\omega}^{T}$ é avaliado em $\left(\hat{\boldsymbol{\beta}}^{T}, \hat{\boldsymbol{\gamma}}^{T}\right)^{T}$. Consequentemente,

$$
\ell_{\max }(\mathbf{q}) \propto \Delta^{T} \ddot{\mathbf{L}}_{\beta \beta}^{-1} \mathbf{q}
$$

Nas subseções seguintes, calcularemos $\ell_{\max }(\mathbf{q})$ sob dois esquemas de perturbação, a perturbação aditiva na resposta e em cada variável explanatória.

\subsubsection{Perturbação aditiva na resposta no modelo simétrico linear heteroscedástico}

Considere inicialmente uma perturbação aditiva na $i$-ésima resposta, isto é $y_{i \omega}=y_{i}+\omega_{i} s_{i}$, em que $s_{i}$ é uma estimativa do desvio padrão de $y_{i}$. Então, temos 
o modelo heteroscedástico perturbado

$$
f_{y_{i}}\left(y_{i} \mid \omega_{i}\right)=\frac{1}{\sqrt{\phi_{i}}} g\left\{\left(y_{i}+\omega_{i} s_{i}-\mathbf{x}_{i}^{T} \beta\right)^{2} / \phi_{i}\right\}=\frac{1}{\sqrt{\phi_{i}}} g\left(u_{i \omega}\right)
$$

em que $u_{i \omega}=\frac{1}{\phi_{i}}\left(y_{i}+\omega_{i} s_{i}-\mathbf{x}_{i}^{T} \boldsymbol{\beta}\right)^{2}=\epsilon_{i \omega}^{2} / \phi_{i}, i=1, \ldots, n$. Então, o logaritmo da função de verossimilhança assume a forma

$$
\mathrm{L}(\theta \mid \omega)=-\frac{1}{2} \sum_{i=1}^{n} \log \left\{\phi_{i}\right\}+\sum_{i=1}^{n} \log \left\{g\left(u_{i \omega}\right)\right\} .
$$

Logo, a matriz $\Delta$ pode ser expressa como $\Delta=\mathrm{X}^{T} \mathrm{D}(\mathrm{a}) \mathrm{D}(\mathrm{s})$, em que $\mathrm{D}(\mathrm{s})=$ $\operatorname{diag}\left\{s_{1}, \ldots, s_{n}\right\}$ e $\mathbf{D}(\mathbf{a})=\operatorname{diag}\left\{a_{1}, \ldots, a_{n}\right\}$, estão definidos na Seção 3.2.1.

$\mathrm{O}$ vetor $\ell_{\text {max }}(\mathbf{q})$ é construído aqui tomando $\mathbf{q}=\mathrm{x}_{i}$, que corresponde ao vetor $n \times 1$ dado por

$$
\begin{aligned}
\ell_{\max }\left(\mathrm{x}_{i}\right) & \propto \Delta^{T} \ddot{\mathrm{L}}_{\beta \beta}^{-1} \mathrm{x}_{i} \\
& \propto \mathrm{D}(\mathrm{s}) \mathrm{D}(\mathrm{a}) \mathrm{X}\left(\mathrm{X}^{T} \mathrm{D}(\mathrm{a}) \mathrm{X}\right)^{-1} \mathbf{x}_{i} .
\end{aligned}
$$

Um grande valor para a $i$-ésima componente de (4.3), $\ell_{\text {max }_{i}}\left(\mathrm{x}_{i}\right)$, indica que a $i$-ésima observação deveria ter uma substancial influência local em $\hat{y}_{i}$. Então, a sugestão é tomar o gráfico de índices do vetor $\left(\ell_{\max _{1}}\left(\mathrm{x}_{1}\right), \ldots, \ell_{\max _{n}}\left(\mathrm{x}_{n}\right)\right)^{T}$ para identificar aquelas observações com influência desproporcional nos próprios valores ajustados.

\subsubsection{Perturbação na variável explanatória no modelo simétrico linear heteroscedástico}

Considere uma perturbação aditiva em uma particular variável explanatória contínua, dada por $x_{i t \omega}=x_{i t}+\omega_{i} s_{t}$, em que $s_{t}$ é um fator de escala. Essa técnica de perturbação leva ao seguinte modelo heteroscedástico perturbado :

$$
f_{y_{i}}\left(y_{i} \mid \omega_{i}\right)=\frac{1}{\sqrt{\phi_{i}}} g\left\{\left(y_{i}-\mathbf{x}_{i \omega}^{T} \boldsymbol{\beta}\right)^{2} / \phi_{i}\right\}=\frac{1}{{\sqrt{\phi_{i}}}} g\left(u_{i \omega}\right),
$$

em que $u_{i \omega}=\frac{1}{\phi_{i}}\left(y_{i}-\mathbf{x}_{i \omega}^{T} \boldsymbol{\beta}\right)^{2}=\epsilon_{i \omega}^{2} / \phi_{i}$, e $\mathbf{x}_{i \omega}=\left(x_{i 1}, \ldots, x_{i t}+s_{t} \omega_{i}, \ldots, x_{i p}\right)^{T}, i=$ $1, \ldots, n$. O logaritmo da função de verossimilhança assume a forma

$$
\mathrm{L}(\boldsymbol{\theta} \mid \boldsymbol{\omega})=-\frac{1}{2} \sum_{i=1}^{n} \log \left\{\phi_{i}\right\}+\sum_{i=1}^{n} \log \left\{g\left(u_{i \omega}\right)\right\},
$$


e depois de algumas manipulações obtemos $\Delta=s_{t}\left\{\mathbf{F D}(\mathbf{e}) \mathbf{D}(\mathrm{g})-\beta_{t} \mathrm{X}^{T} \mathbf{D}(\mathbf{a})\right\}$, em que $\mathrm{F}$ é uma matriz $p \times n$ de zeros com uns na $t$-ésima linha. Similarmente ao caso da perturbação na resposta o sugerido aqui é avaliar a maior curvatura em $\mathrm{q}=\mathrm{x}_{i}$, que leva ao seguinte :

$$
\begin{aligned}
\mathrm{C}_{\max }\left(\mathrm{x}_{i}\right) & =\left|\ell_{\max }^{T} \ddot{\mathbf{f}} \ell_{\max }\right| \\
& =2\left|\mathbf{x}_{i}^{T} \ddot{\mathbf{L}}_{\beta \beta}^{-1} \Delta \Delta^{T} \mathbf{L}_{\beta \beta}^{-1} \mathbf{x}_{i}\right|
\end{aligned}
$$

e consequentemente

$$
\begin{aligned}
\ell_{\max }\left(\mathbf{x}_{i}\right) & \propto \Delta^{T} \ddot{\mathbf{L}}_{\beta \beta}^{-1} \mathbf{x}_{i} \\
& \propto\left\{\mathbf{F D}(\mathbf{e}) \mathbf{D}(\mathrm{g})-\beta_{t} \mathbf{X}^{T} \mathbf{D}(\mathbf{a})\right\}^{T}\left(\mathbf{X}^{T} \mathbf{D}(\mathbf{a}) \mathbf{X}\right)^{-1} \mathbf{x}_{i}
\end{aligned}
$$

Para avaliar em quais valores observados de $\mathrm{x}_{t}$ a predição é mais sensível sob pequenas mudanças em $\mathrm{x}_{t}$, podemos construir o gráfico de $\mathrm{C}_{\text {max }}\left(\mathrm{x}_{i}\right)$ contra $x_{i t}$. $\mathrm{O}$ gráfico de índices do vetor $\left(\ell_{\max _{1}}\left(\mathrm{x}_{1}\right), \ldots, \ell_{\max }\left(\mathrm{x}_{n}\right)\right)^{T}$ pode indicar aquelas observações para as quais uma pequena perturbação em $\mathrm{x}_{t}$ leva a mudanças substanciais na predição.

\subsection{Ponto de alavanca generalizado no modelo simétrico de regressão}

Seja $\mathrm{y}=\left(y_{1}, \ldots, y_{n}\right)^{T}$ o vetor de respostas observadas as quais têm função de probabilidade de densidade $f(\mathbf{y} ; \boldsymbol{\theta})$, sendo $\boldsymbol{\theta}$ um vetor $q$-dimensional. Se denotarmos por $\hat{\theta}=\theta(\mathrm{y})$ a estimativa de máxima verossimilhança de $\theta$ e por $\boldsymbol{\mu}$ o vetor de valores esperados, então $\hat{y}=\boldsymbol{\mu}(\hat{\theta})$ é o vetor de respostas preditas. A principal idéia por trás do conceito de ponto de alavanca (veja, por exemplo, Hoaglin e Welsch, (1978); Cook e Weisberg, (1982); Emerson, Hoaglin e Kempthorne, (1984); St. Laurent e Cook, (1992) e Wei, Hu e Fung, (1998)) é conhecer a influência de $y_{i}$ no próprio valor predito. Essa influência pode șer bem representada pela derivada $\partial \hat{y}_{i} / \partial y_{i}$ que é igual a $h_{i i}$ no caso normal linear, em que $h_{i i}$ é o $i$-ésimo elemento da diagonal principal da matriz de projeção $H=X\left(X^{T} X\right)^{-1} X^{T}$ e $X$ é a matriz modelo. Extensões para modelos de regressão mais gerais têm sido propostas, por 
exemplo, por St. Laurent e Cook (1992) e Wei, Hu e Fung, (1998) quando $\theta$ é irrestrito e por Paula $(1993,1995,1999 b)$ quando $\theta$ é restrito em desigualdades lineares. Em particular, se denotarmos por $\mathrm{L}(\boldsymbol{\theta} ; \mathrm{y})$ o logaritmo da função de verossimilhança de $\theta \in \mathbb{R}^{q}$ e por $\hat{\theta}(\mathrm{y})$ a estimativa que maximiza $\mathrm{L}(\boldsymbol{\theta} ; \mathrm{y})$, segue de Wei, Hu e Fung (1998) que a matriz $(n \times n)\left(\partial \hat{\mathbf{y}} / \partial \mathbf{y}^{T}\right)$ de pontos de alavanca pode ser expressa na forma

$$
\mathrm{GL}(\hat{\theta})=\left.\left\{\left(\mathrm{D}_{\theta}\right)\left(-\ddot{\mathrm{L}}_{\theta \theta}\right)^{-1}\left(\ddot{\mathrm{L}}_{\theta \mathrm{y}}\right)\right\}\right|_{\theta=\hat{\boldsymbol{\theta}}_{(\mathrm{y})}},
$$

em que $\mathbf{D}_{\theta}=\partial \boldsymbol{\mu} / \partial \boldsymbol{\theta}^{T}, \ddot{\mathrm{L}}_{\theta \theta}=\partial^{2} \mathrm{~L}(\boldsymbol{\theta} ; \mathrm{y}) / \partial \boldsymbol{\theta} \partial \boldsymbol{\theta}^{T}$ e $\ddot{\mathrm{L}}_{\theta \mathbf{y}}=\partial^{2} \mathrm{~L}(\boldsymbol{\theta} ; \mathrm{y}) / \partial \boldsymbol{\theta} \partial \mathrm{y}^{T} . \mathrm{A}$ expressão (4.5) generaliza a definição de pontos de alavanca generalizados dada em St. Laurent e Cook (1992).

Sendo $\mathrm{D}_{\theta}=\left(\mathrm{D}_{\beta}, 0\right)$, e desde que

$$
\ddot{\mathrm{L}}_{\beta y}=\frac{1}{\phi} \mathbf{D}_{\beta}^{T} \mathbf{D}(\mathbf{a}) \text { e } \ddot{\mathbf{L}}_{\phi y}=-\frac{2}{\phi^{2}} \mathbf{b}^{T},
$$

então usando a expressão (4.5) a matriz generalizada de pontos de alavanca toma a forma

$$
\mathrm{GL}(\hat{\theta})=\mathrm{GL}_{\beta}(\hat{\theta})+\mathrm{GL}_{\phi}(\hat{\theta})
$$

em que

$$
\begin{gathered}
\mathrm{GL}_{\beta}(\hat{\boldsymbol{\theta}})=\mathbf{D}_{\hat{\beta}} \hat{\mathrm{M}}^{-1} \mathrm{D}_{\hat{\beta}}^{T} \mathrm{D}(\hat{\mathrm{a}}) \quad \mathrm{e} \\
\mathrm{GL}_{\phi}(\hat{\boldsymbol{\theta}})=\frac{4}{\hat{\mathrm{E}} \hat{\phi}^{3}} \mathbf{D}_{\hat{\beta}} \hat{\mathrm{M}}^{-1} \mathbf{D}_{\hat{\beta}}^{T} \hat{\mathrm{b}} \hat{\mathrm{b}}^{T}\left\{\mathrm{I}_{n}-\mathrm{GL}_{\beta}(\hat{\boldsymbol{\theta}})\right\}
\end{gathered}
$$

em que $\hat{\mathbf{M}}=\mathbf{D}_{\hat{\beta}}^{T} \mathbf{D}(\hat{\mathbf{a}}) \mathbf{D}_{\hat{\beta}}+2\left[\hat{\mathbf{s}}^{T}\right]\left[\mathbf{D}_{\hat{\beta} \hat{\beta}}\right], \mathbf{I}_{n}$ sendo a matriz identidade de ordem $n, \mathrm{D}(\mathrm{a})$ e $\mathrm{E}$ estão definidos na seção 2.2.1. Uma interpretação interessante para (4.6) pode ser obtida se considerarmos o procedimento de estimação de mínimos quadrados ao invés de máxima verossimilhança, considerando a função objetivo

$$
Q(\boldsymbol{\beta})=\frac{1}{2 \sigma^{2}} \sum_{i=1}^{n} a_{i}\left\{y_{i}-\mu_{i}(\boldsymbol{\beta})\right\}^{2},
$$

em que $\operatorname{Var}\left(y_{i}\right)=\frac{\sigma^{2}}{a_{i}}$ e os $a_{i}$ 's são constantes positivas. Então, usando a expressão geral (2.2) de Wei, Hu e Fung (1998) encontramos $\mathrm{GL}(\hat{\boldsymbol{\theta}})=\mathrm{GL}_{\beta}(\hat{\boldsymbol{\theta}}) \operatorname{com} s_{i}=$ 
$-a_{i} e_{i}$. Isto é, o procedimento de mínimos quadrados leva em conta somente a influência da estimativa do parâmetro de locação na medida de alavanca, enquanto o de máxima verossimilhança também tende a considerar a influência da estimativa do parâmetro de escala. Quando o parâmetro de dispersão $\phi$ é conhecido é fácil mostrar que $\mathrm{GL}(\hat{\boldsymbol{\theta}})=\mathrm{GL}_{\beta}(\hat{\boldsymbol{\theta}})$. Contudo, para o caso normal, desde que $\mathrm{D}_{\hat{\beta}}^{T} \hat{\mathrm{b}}=0$ a influência de $\hat{\phi}$ na matriz generalizada de pontos de alavanca anula-se e $\operatorname{GL}(\hat{\theta})$ reduz-se à matriz jacobiana de pontos de alavanca

$$
\hat{\mathbf{J}}=\mathrm{D}_{\hat{\beta}}\left\{\mathbf{D}_{\hat{\beta}}^{T} \mathbf{D}_{\hat{\beta}}-\left[\hat{\mathbf{e}}^{T}\right]\left[\mathbf{D}_{\hat{\beta} \hat{\beta}}\right]\right\}^{-1} \mathbf{D}_{\hat{\beta}}^{T}
$$

St. Laurent and Cook (1992) comparam (4.7) com a matriz de pontos de alavanca do plano tangente definida por $\hat{\mathbf{H}}=\mathbf{D}_{\hat{\beta}}\left(\mathbf{D}_{\hat{\beta}}^{T} \mathbf{D}_{\hat{\beta}}\right)^{-1} \mathbf{D}_{\hat{\beta}}^{T}$, que é a matriz de projeção ortogonal no subespaço gerado pelas colunas da matriz $\mathbf{D}_{\hat{\beta}}$. Nesse caso, seguem as propriedades $0 \leqslant \hat{h}_{i i} \leqslant 1, \sum \hat{h}_{i i}=p$ e que $\hat{h}_{k k}=1$ implica em $\hat{h}_{i k}=0$ para $i \neq k$. Essas propriedades não são garantidas para $\hat{j}_{i i}$, o $i$-ésimo elemento da diagonal de $\hat{\mathrm{J}}$. Podemos ter, por exemplo, $\hat{j}_{i i}>1$ chamado superalavanca.

\subsubsection{Caso linear homoscedástico}

Considere agora o caso linear homoscedástico em que $y_{i}=\mathbf{x}_{i}^{T} \boldsymbol{\beta}+\epsilon_{i}$ e seja $\mathbf{X}$ a matriz modelo com linhas $\mathbf{x}_{i}^{T}, i=1, \ldots, n$. Segue que $\mathrm{D}_{\beta}=\mathbf{X}$ e $\mathbf{D}_{\beta \beta}=0$ de modo que a matriz generalizada de pontos de alavanca assume uma forma simplificada

$$
\mathrm{GL}(\hat{\theta})=\hat{\mathrm{H}}+\frac{4}{\hat{\mathrm{E}} \hat{\phi}^{3}} \hat{\mathrm{H}} \mathrm{D}^{-1}(\hat{\mathbf{a}}) \hat{\mathrm{b}} \hat{\mathrm{b}}^{T}\left\{\mathrm{I}_{n}-\hat{\mathrm{H}}\right\}
$$

em que $\hat{\mathrm{H}}=\mathrm{X}\left\{\mathrm{X}^{T} \mathbf{D}(\hat{\mathrm{a}}) \mathrm{X}\right\}^{-1} \mathrm{X}^{T} \mathrm{D}(\hat{\mathrm{a}})$. Entretanto, se os $a_{i}^{\prime} s$ são constantes positivas $\hat{\mathrm{H}}$ pode ser interpretada como a matriz de projeção ortogonal em $C\left(\mathrm{XD}^{1 / 2}(\hat{\mathrm{a}})\right)$, que denota o subespaço gerado pelas colunas da matriz $\mathrm{XD}^{1 / 2}(\hat{\mathrm{a}})$. Quando $a_{i}=1$, $\forall i$, tem-se $\mathbf{H}=\mathbf{X}\left(\mathbf{X}^{T} \mathbf{X}\right)^{-1} \mathbf{X}^{T}$. 
4.3.2 Relação entre a medida de influência e a matriz de pontos de alavanca generalizados

Usando o esquema de perturbação aditiva na resposta encontramos que $\hat{\Delta}^{T}=$ $\left[(1 / \hat{\phi}) \mathbf{D}(\hat{\mathbf{a}}) \mathbf{D}_{\hat{\beta}},-\left(2 / \hat{\phi}^{2}\right) \hat{\mathbf{b}}\right]$. Então, podemos expressar

$$
\mathrm{B}=\frac{1}{\hat{\phi}}\left[\mathbf{D}(\hat{\mathbf{a}}) \mathrm{GL}(\hat{\theta})+\frac{4 \mathrm{bb}^{T}}{\hat{\phi}^{3} \hat{\mathrm{E}}}\left\{\mathrm{I}-\mathrm{GL}_{\beta}(\hat{\theta})\right\}\right] .
$$

Em particular, quando $\phi$ é fixado, a matriz generalizada de pontos de alavanca $\mathrm{GL}(\hat{\theta})$ reduz-se a

$$
\begin{aligned}
\mathrm{GL}(\hat{\theta}) & =-\mathrm{D}_{\hat{\beta}} \ddot{\mathrm{L}}_{\hat{\beta} \hat{\beta}}^{-1} \mathbf{D}_{\hat{\beta}}^{T} \mathrm{D}(\hat{\mathrm{a}}), \quad \mathrm{e} \\
\mathrm{B} & =-\Delta^{T} \ddot{\mathrm{L}}_{\hat{\beta} \hat{\beta}}^{-1} \Delta \\
& =-\frac{1}{\hat{\phi}^{2}} \mathrm{D}(\hat{\mathbf{a}}) \mathbf{D}_{\hat{\beta}} \ddot{\mathrm{L}}_{\hat{\beta} \hat{\beta}}^{-1} \mathbf{D}_{\hat{\beta}}^{T} \mathbf{D}(\hat{\mathbf{a}}) \\
& =\frac{1}{\hat{\phi}} \mathbf{D}(\hat{\mathbf{a}}) \mathbf{G L}(\hat{\theta}) .
\end{aligned}
$$

Nesse caso, a medida de influência $b_{i i}$ assume a forma simples

$$
b_{i i}=\frac{\hat{a}_{i}}{\hat{\phi}} \mathrm{GL}_{i i}(\hat{\boldsymbol{\theta}}),
$$

em que $a_{i}=-2\left\{W_{g}\left(u_{i}\right)+2 u_{i} W_{g}^{\prime}\left(u_{i}\right)\right\}$. Então, pela Tabela 2.1 temos que $a_{i}=1$ para o caso normal e $a_{i}=(\nu+1)\left(\nu-3 u_{i}\right) /\left(\nu+u_{i}\right)^{2}$ para a distribuição $t$-Student com $\nu$ graus de liberdade. A expressão (4.8) pode ser usada para avaliar a influência local total da $i$-ésima observação na estimativa $\hat{\beta}$.

\subsubsection{Caso linear heteroscedástico}

Para o caso do modelo simétrico linear heteroscedástico pode haver interesse em duas medidas de pontos de alavanca, a influência de $y_{i}$ no seu próprio valor médio predito $\hat{y}_{i}$, representado por $\partial \hat{y}_{i} / \partial y_{i}$ e a influência de $y_{i}$ em $\hat{\phi}_{i}$, denotada por $\frac{\partial \hat{\phi}_{i}}{\partial y_{i}}$ em que $\phi=\left(\phi_{1}, \ldots, \phi_{n}\right)^{T}$. Então, pela expressão (4.5) a matriz generalizada de pontos de alavanca $\partial \hat{y} / \partial \mathbf{y}^{T}$ toma a forma

$$
\mathrm{GL}^{\mu}(\hat{\theta})=\mathrm{GL}_{\beta}^{\mu}(\hat{\theta})+\mathrm{GL}_{\gamma}^{\mu}(\hat{\theta})
$$


Desde que $\mathrm{D}_{\theta}=(\mathrm{X}, 0), \ddot{\mathrm{L}}_{\beta y}=\mathrm{X}^{T} \mathrm{D}(\mathrm{a})$ e $\ddot{\mathbf{L}}_{\gamma y}=-2 \mathrm{Z}^{T} \mathrm{D}(\mathrm{b})$, temos que

$$
\begin{aligned}
\mathrm{GL}_{\beta}^{\mu}(\theta) & =\mathrm{X}\left\{\mathrm{X}^{T} \mathrm{D}(\mathrm{a}) \mathrm{X}\right\}^{-1} \mathrm{X}^{T} \mathrm{D}(\mathrm{a}) \text { e } \\
\mathrm{GL}_{\gamma}^{\mu}(\theta) & =-\mathrm{XAE}^{-1} \mathrm{~A}^{T} \mathrm{X}^{T} \mathrm{D}(\mathrm{a})+2 \mathrm{XAE}^{-1} \mathrm{Z}^{T} \mathrm{D}(\mathrm{b})
\end{aligned}
$$

sendo as quantidades definidas na Seção 3.2.1.

Se a matriz de informação esperada de Fisher é usada no lugar de $-\ddot{\mathbf{L}}_{\theta \theta}$, então a matriz de pontos de alavanca $\mathrm{GL}^{\mu}(\boldsymbol{\theta})$ pode ser expressa como $\mathrm{GL}^{\mu}(\boldsymbol{\theta})=$ $\mathrm{X}\left(\mathrm{X}^{T} \hat{\mathbf{W}}_{1} \mathrm{X}^{T}\right)^{-1} \mathrm{XD}(\hat{\mathrm{a}})$. Similarmente, a matriz de pontos de alavanca $\frac{\partial \hat{\phi}}{\partial \mathbf{y}^{T}}$ pode ser expressa como

$$
\mathrm{GL}^{\phi}(\hat{\theta})=\mathrm{GL}_{\gamma}^{\phi}(\hat{\theta})+\mathrm{GL}_{\mu}^{\phi}(\hat{\theta})
$$

em que

$$
\begin{aligned}
\mathrm{GL}_{\gamma}^{\phi}(\boldsymbol{\theta}) & =-2 \mathrm{Z}\left\{\mathbf{Z}^{T} \mathrm{D}(\mathbf{c}) \mathbf{Z}\right\}^{-1} \mathbf{Z}^{T} \mathbf{D}(\mathbf{b}) \text { e } \\
\mathrm{GL}_{\beta}^{\phi}(\boldsymbol{\theta}) & =2 \mathrm{ZAE}^{-1} \mathbf{A}^{T} \mathbf{Z}^{T} \mathbf{D}(\mathbf{b})-\mathbf{Z A E}^{-1} \mathbf{X}^{T} \mathrm{D}(\mathbf{a})
\end{aligned}
$$

em que $\mathbf{A}=2\left\{\mathbf{Z}^{T} \mathbf{D}(\mathbf{c}) \mathbf{Z}\right\}^{-1} \mathbf{Z}^{T} \mathbf{D}(\mathbf{b}) \mathbf{X}^{T}, \mathbf{E}=-\mathbf{X}^{T} \mathbf{D}(\mathbf{a}) \mathbf{X}+2 \mathbf{X}^{T} \mathbf{D}(\mathbf{b}) \mathbf{Z A}$. Se $-\ddot{\mathbf{L}}_{\theta \theta}$ é substituído pela informação esperada de Fisher segue que $\operatorname{GL}_{\gamma}^{\phi}(\hat{\boldsymbol{\theta}})=$ $-2 \mathrm{Z}\left(\mathbf{Z}^{T} \hat{\mathbf{W}}_{2} \mathbf{Z}^{T}\right)^{-1} \mathbf{Z}^{T} \mathbf{D}(\hat{\mathbf{b}})$.

\subsection{Aplicações não-linear (Coelhos)}

Na Seção 2.4 iniciamos a discussão sobre a modelagem não-linear para os dados dos coelhos. Paula, Cysneiros e Galea (2003) observam que os pontos 1, 2 e 3 aparecem como pontos de alavanca nos três modelos mostrando a dificuldade de predição na resposta para animais jovens (vide, Figura 4.4). O modelo $t$-Student destaca menos observações nos gráficos de índices de $\mathrm{C}_{i}$ do que os modelos logísticoII e normal (vide Figuras 4.1-4.3). Aqui, os animais jovens tendem a ser mais influentes nas estimativas dos parâmetros. A linha pontilhada nos gráficos de $\mathrm{GL}_{i i}$ representa o gráfico de índices de $\hat{h}_{i i}$ (ponto de alavanca do plano tangente) que são negligenciáveis, como esperado, para o caso normal, pois a curvatura intrínseca é não significativa, mas difere de valores nos modelos $t$-Student e modelo logístico-II. 
A eliminação das observações 16 e 17 produz maiores mudanças nas estimativas do modelo normal do que nas estimativas do modelo $t$-Student e do modelo logísticoII (vide Tabela 4.1). Eliminando os pontos influentes e de alta alavanca (vide Tabela 4.2) ocorrem mais variações, sob o modelo normal do que sob os modelos t-Student e logístico-II. Nossa principal conclusão, desta análise de diagnóstico, é que o modelo $t$-Student com 10 graus de liberdade parece ser mais robusto contra o esquema de perturbação heteroscedástico do que o modelo normal, previamente usado para analisar esse conjunto de dados.

Tabela 4.1 Mudanças (em \%) nas estimativas dos parâmetros dos modelos ajustados aos dados dos coelhos depois de excluídas as observações (16,17).

\begin{tabular}{c|ccc}
\hline Parâmetro & Normal & $t_{10}$ & Logístico-II \\
\hline$\alpha$ & $-0,24$ & $-0,13$ & $-0,11$ \\
& $(-13,68)$ & $(-9,83)$ & $(-9,29)$ \\
$\beta$ & $-4,41$ & $-2,51$ & $-2,18$ \\
& $(-14,77)$ & $(-9,90)$ & $(-9,15)$ \\
$\gamma$ & $-5,60$ & $-3,35$ & $-2,88$ \\
& $(-14,56)$ & $(-10,06)$ & $(-9,33)$ \\
$\phi$ & $-24,38$ & $-18,87$ & $-18,18$ \\
& $(-23,29)$ & $(-17,71)$ & $(-17,01)$ \\
\hline
\end{tabular}

Tabela 4.2 Mudanças (em \%) nas estimativas dos parâmetros dos modelos ajustados aos dados dos coelhos depois de excluidas as observações $(1,2,3,4,5,16,17)$.

\begin{tabular}{c|ccc}
\hline Parâmetro & Normal & $t_{10}$ & Logístico-II \\
\hline$\alpha$ & $-0,3$ & $-0,13$ & $-0,08$ \\
& $(-7,38)$ & $(1,34)$ & $(2,63)$ \\
$\beta$ & $-5,97$ & $-2,76$ & $-1,92$ \\
& $(11,33)$ & $(25,16)$ & $(27,64)$ \\
$\gamma$ & $-10,75$ & $-5,19$ & $-3,51$ \\
& $(47,56)$ & $(66,19)$ & $(69,55)$ \\
$\phi$ & $-40,98$ & $-32,14$ & $-31,18$ \\
& $(-37,83)$ & $(-28,53)$ & $(-27,51)$ \\
\hline
\end{tabular}


Figura 4.1 Gráficos de índices de $\mathrm{C}_{i}$ sob o modelo normal (esquerda), $t$-Student com 10 g.l. (direita) e logístico-II (abaixo) ajustados aos dados dos coelhos da Tabela C.1.
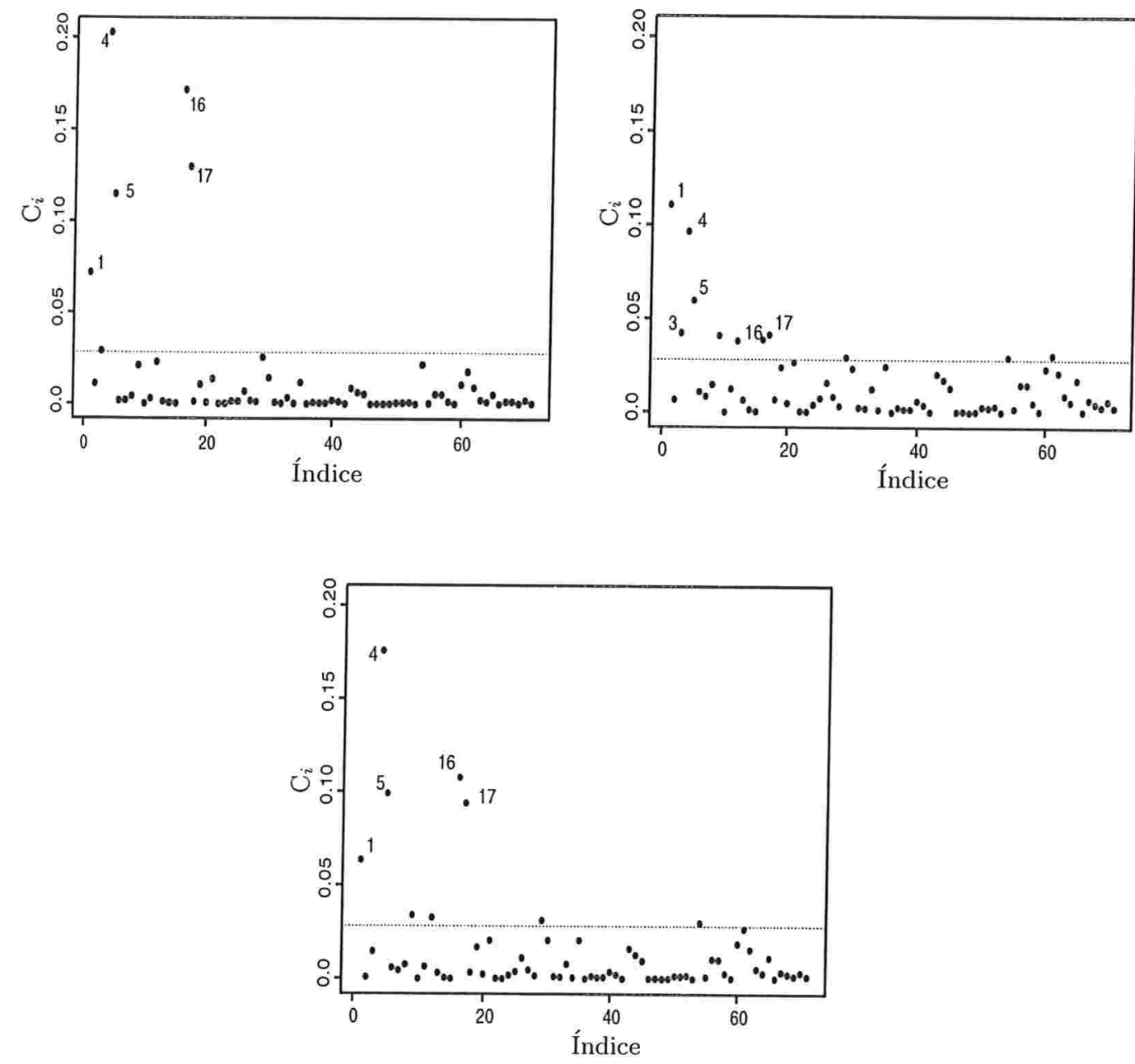
Figura 4.2 Gráficos de índices de $\mathrm{C}_{i}(\beta)$ sob o modelo normal (esquerda), $t$-Student com 10 g.l. (direita) e logístico-II (abaixo) ajustados aos dados dos coelhos da Tabela C.1.
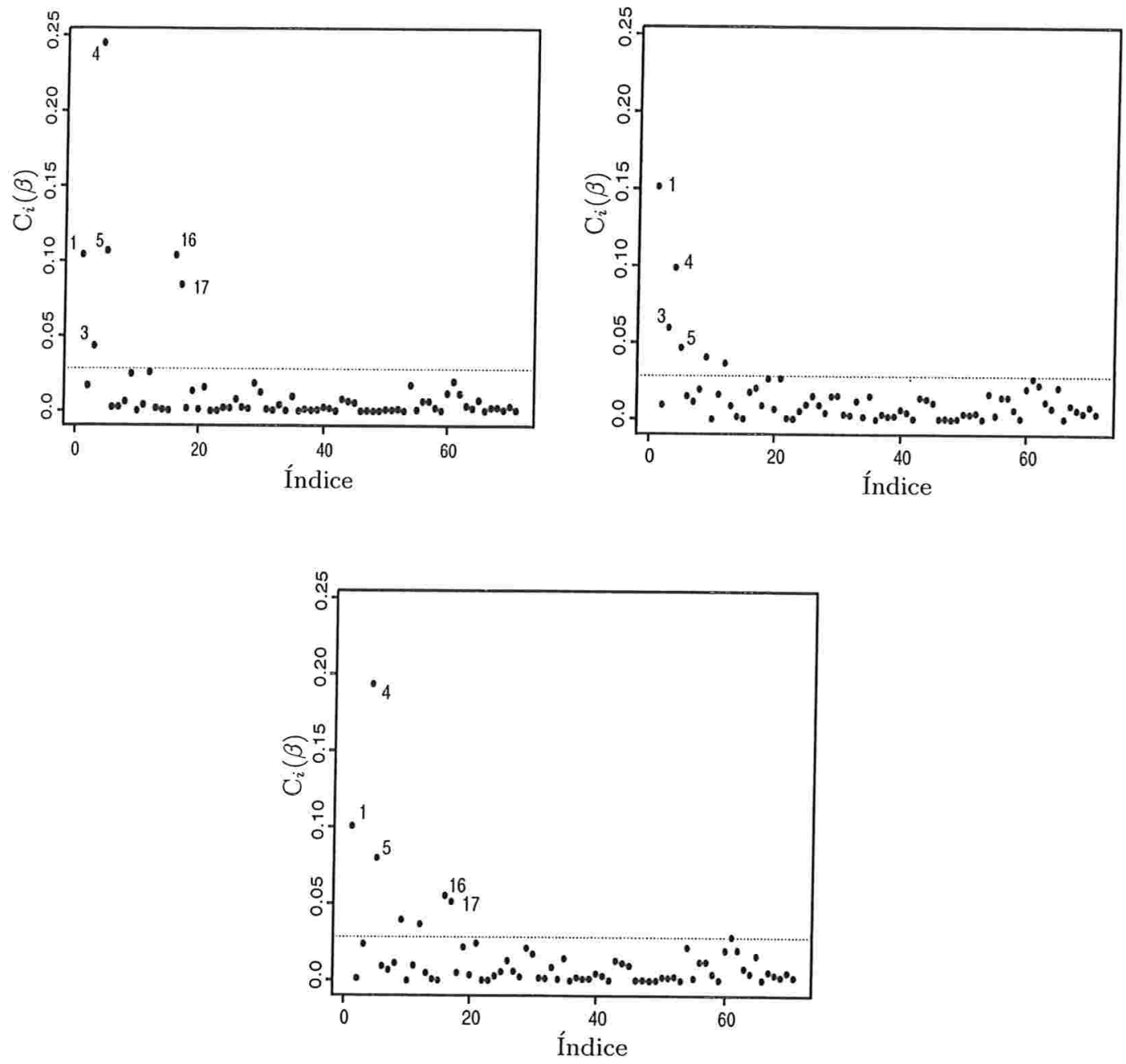
Figura 4.3 Gráficos de índices de $\mathrm{C}_{i}(\phi)$ sob o modelo normal (esquerda), $t$-Student com 10 g.l. (direita) e logístico-II (abaixo) ajustados aos dados dos coelhos da Tabela C.1.
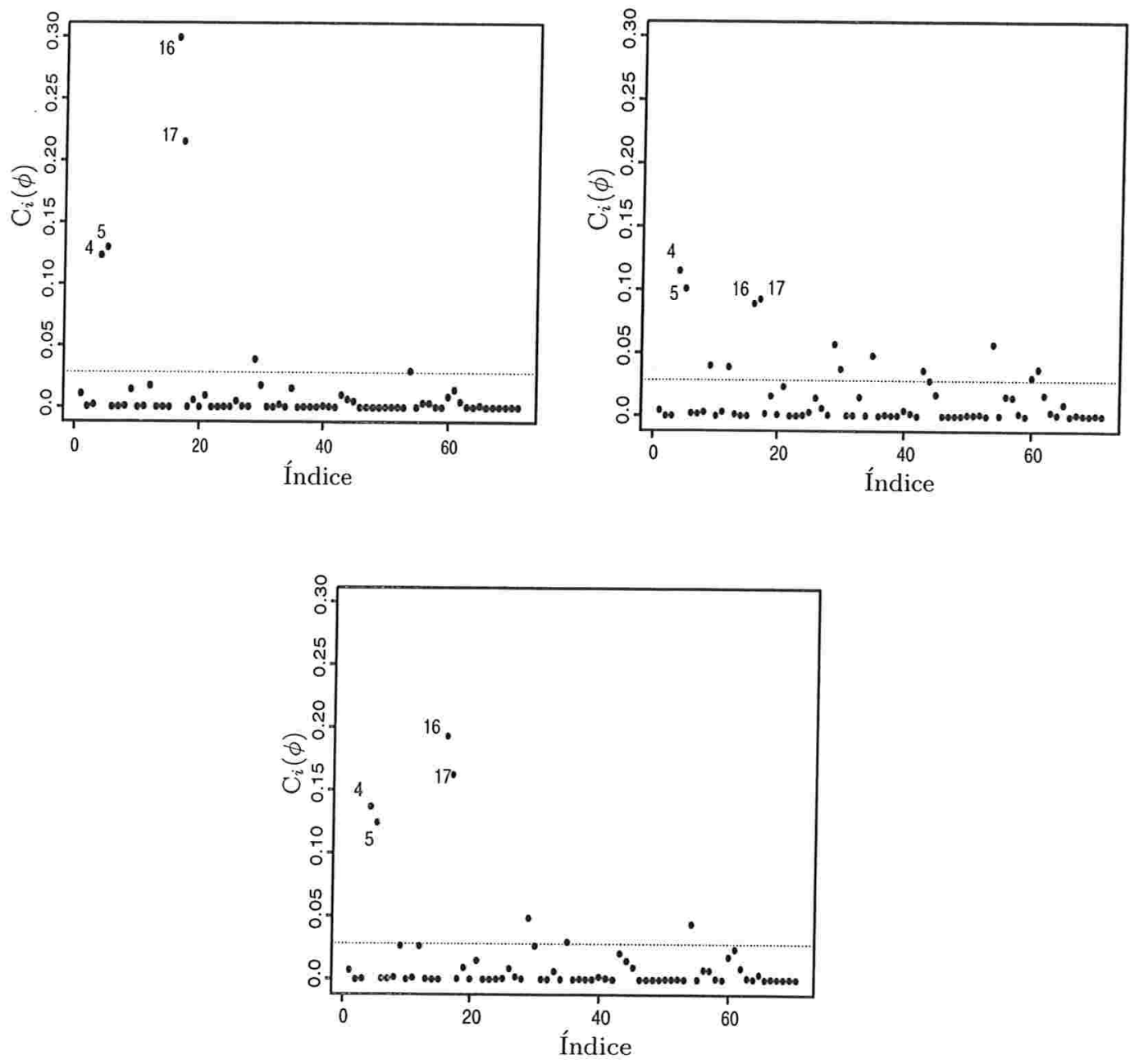
Figura 4.4 Gráficos de pontos de alavanca generalizados contra idade sob o modelo normal (esquerda), t-Student com 10 g.l. (direita) e logístico-II (abaixo) ajustados aos dados dos coelhos da Tabela C.1.
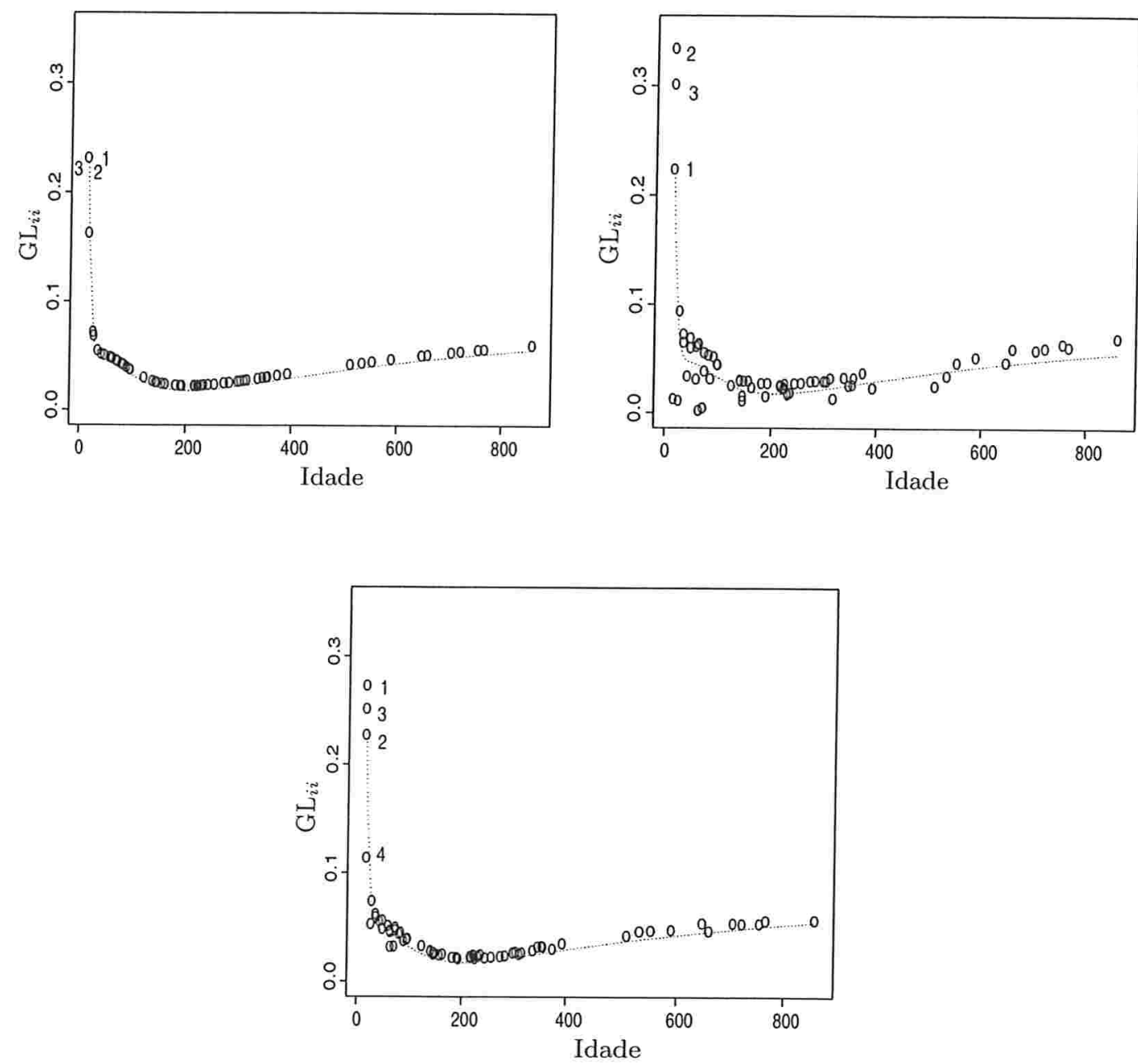


\subsection{Aplicação heteroscedástica}

Prosseguindo a análise iniciada na Seção 3.4, em que se ajustou um modelo simétrico heteroscedástico para explicar o tempo gasto no serviço $(y)$ segundo o número de bebidas estocadas $\left(x_{1}\right)$ e a distância pecorrida pelo motorista $\left(x_{2}\right)$, iremos, agora, aplicar alguns procedimentos de diagnóstico de influência. A Figura 4.5 descreve o gráfico de índices de $\mathrm{C}_{\max }$ quando $x_{1}$ é perturbado. Podemos notar, pelo gráfico, uma dificuldade na predição das observações 9 e 22 para todos os modelos. Logo, pequenas mudanças nos valores de $x_{1}$ levam a mudanças substanciais na predição das observações 9 e 22. A Figura 4.6 mostra o gráfico de índices de $\mathrm{C}_{\max }$ quando $x_{2}$ é perturbado. Nesse caso a observação 9 aparece com maior destaque. O gráfico de índices dos pontos de alavanca generalizados (Figura 4.7) não indica muitas observações. Nota-se uma certa robustez do modelo logístico-II. As Tabelas 4.3 e 4.4 mostram as variações nas estimativas depois de excluídas as observações 9 e 22, respectivamente. Observa-se nesses casos, uma certa robustez do modelo $t$-Student em relação à estimativa de $\delta_{2}$ que parece indicar que o modelo $t$-Student é o mais adequado dentre os modelos estudados para esses dados. 
Figura 4.5 Gráfico de $\mathrm{C}_{\max }$ contra $x_{1}$ quando $x_{1}$ é perturbado sob o modelo normal (esquerda), t-Student com 4 g.l. (direita) e logístico-II (abaixo) ajustados aos dados de estoque da Tabela D.1.
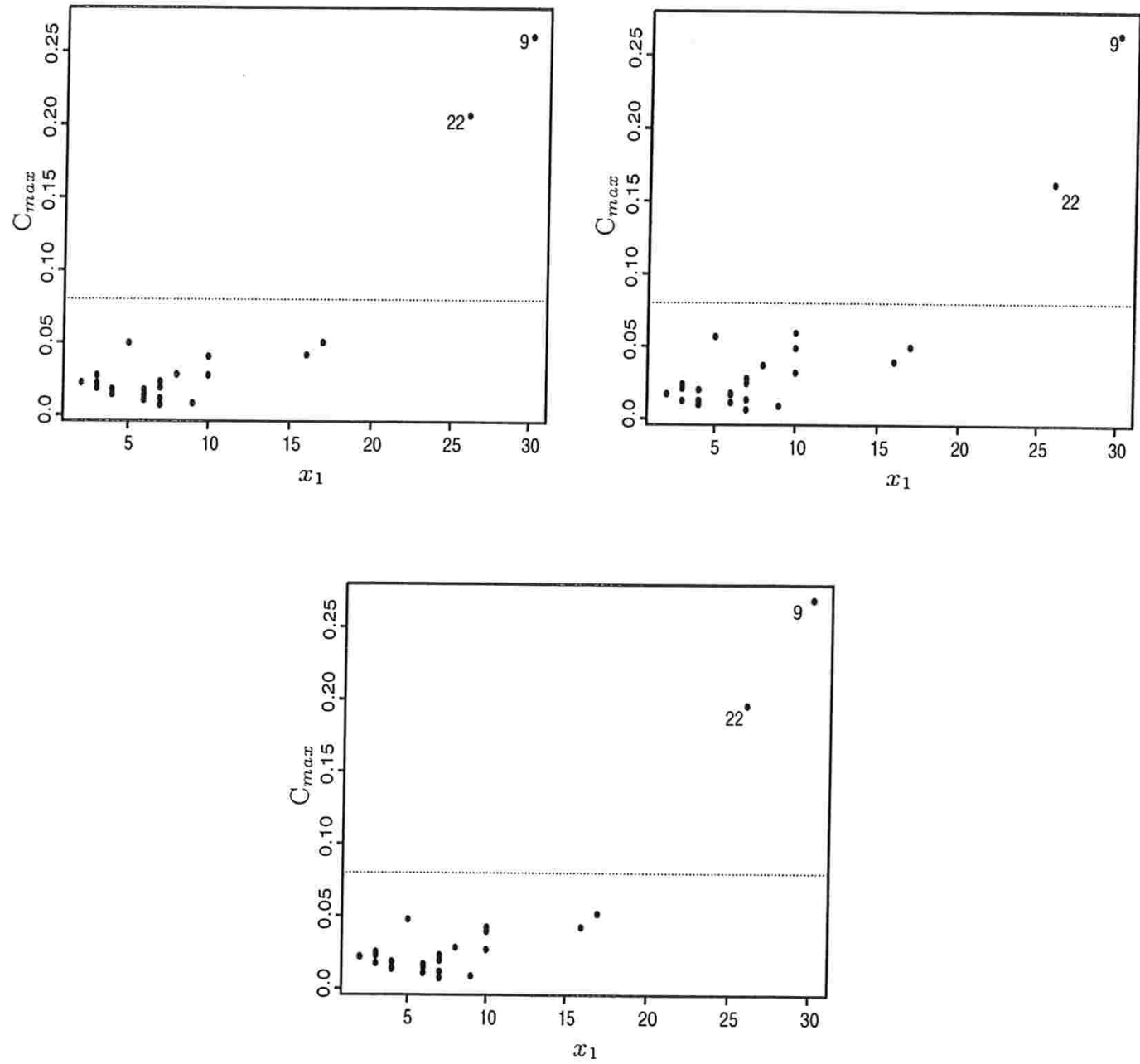
Figura 4.6 Gráfico de $\mathrm{C}_{\max }$ contra $x_{2}$ quando $x_{2}$ é perturbado sob o modelo normal (esquerda), $t$-Student com 4 g.l. (direita) e logístico-II (abaixo) ajustados aos dados de estoque da Tabela D.1.
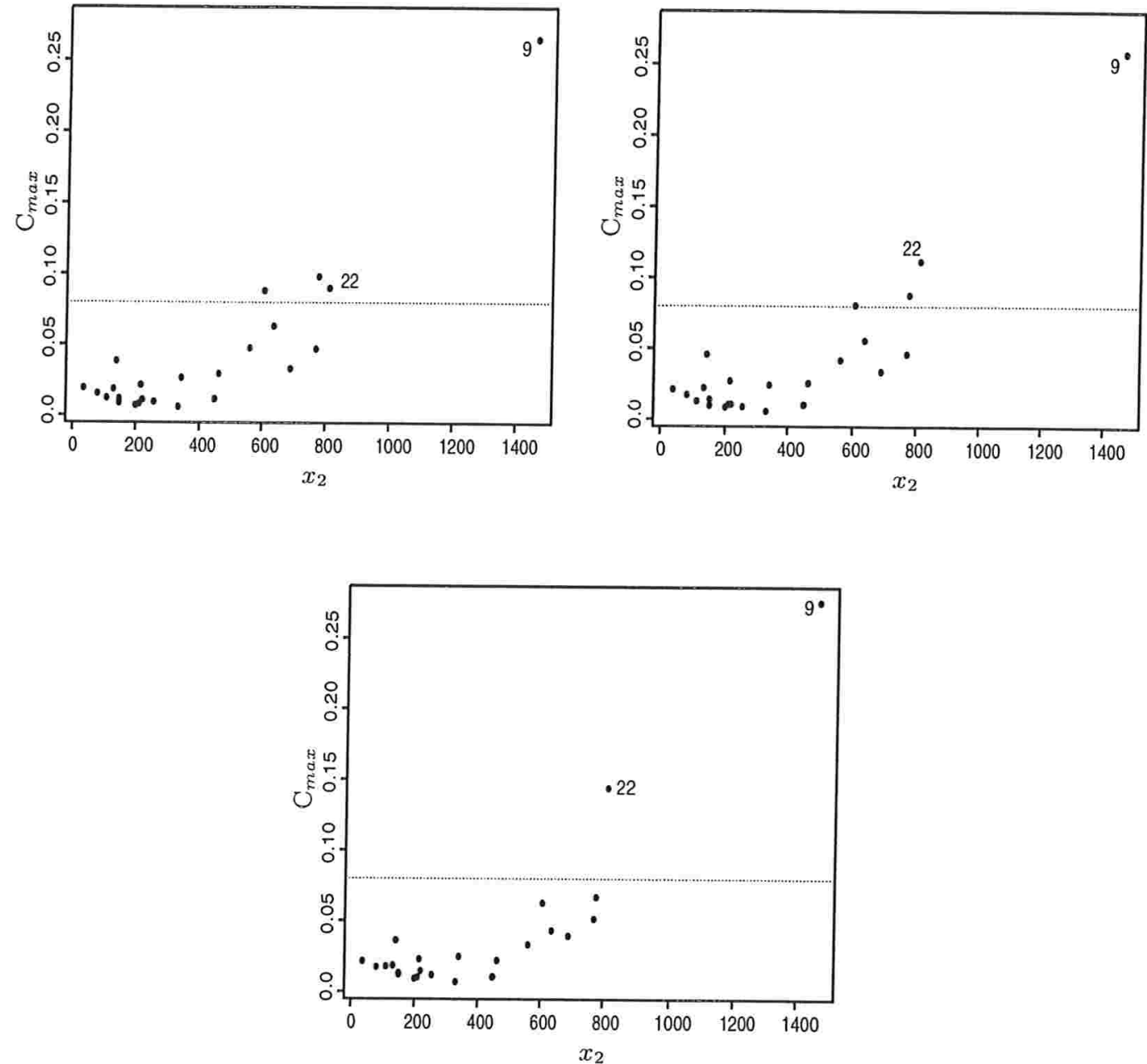
Figura 4.7 Gráfico de índices dos pontos de alavanca generalizados sob o modelo normal (esquerda), $t$-Student com 4 g.l. (direita) e logístico-II (abaixo) ajustados aos dados de estoque da Tabela D.1.
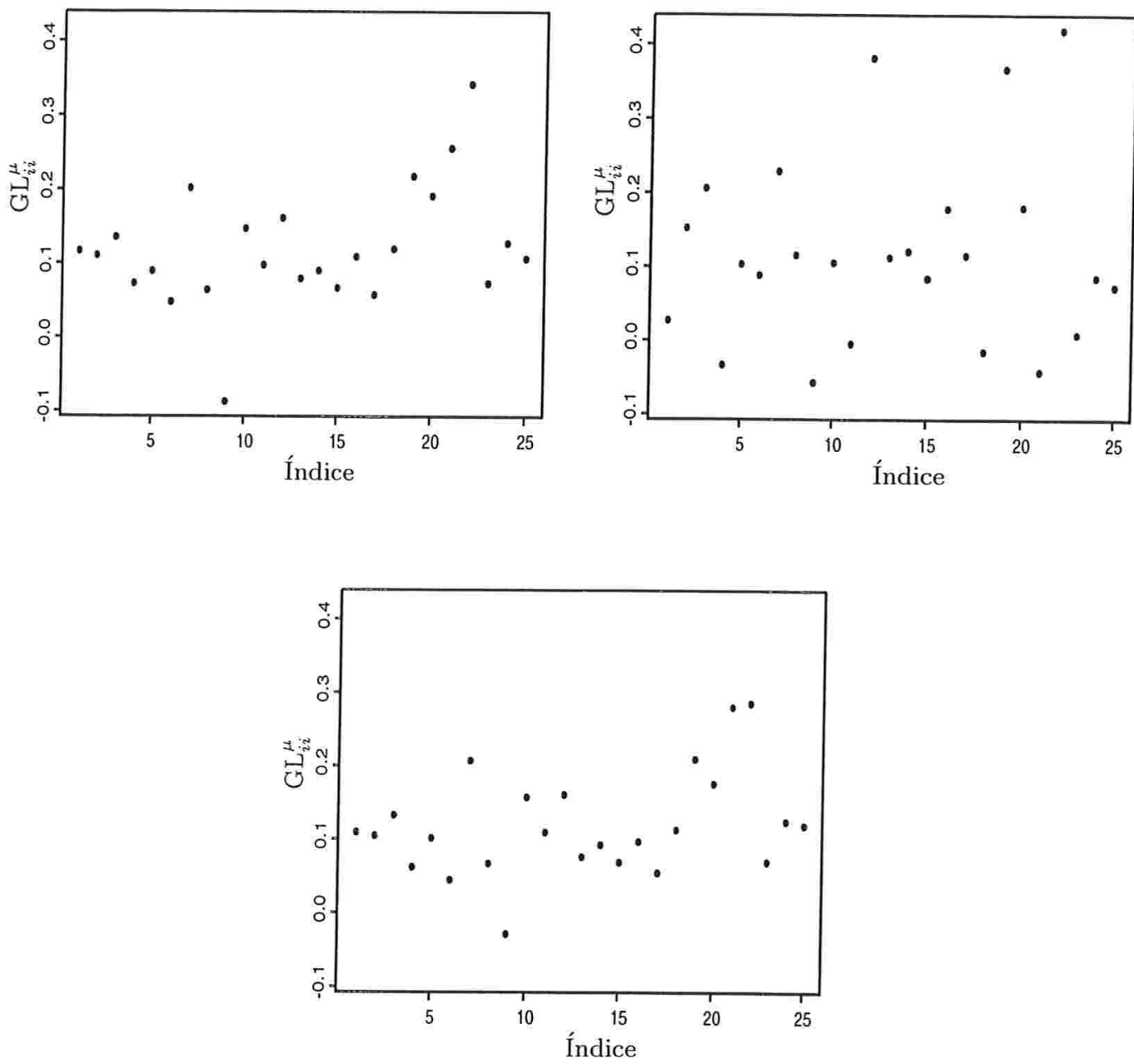
Tabela 4.3 Mudanças (em \%) nas estimativas dos parâmetros dos modelos ajustados aos dados de estoque depois de excluída a observação 9.

\begin{tabular}{c|ccc}
\hline Parâmetro & Normal & $t_{4}$ & Logístico-II \\
\hline$\beta_{0}$ & 2,12 & 3,21 & 3,17 \\
& $(0,00)$ & $(2,55)$ & $(1,32)$ \\
$\beta_{1}$ & $-0,17$ & $-0,33$ & $-0,40$ \\
& $(-5,75)$ & $(-0,81)$ & $(-3,11)$ \\
$\beta_{2}$ & $-4,48$ & $-4,04$ & $-4,28$ \\
& $(-4,93)$ & $(-1,35)$ & $(-3,03)$ \\
$\alpha$ & $-6,56$ & $-3,09$ & $-12,66$ \\
& $(3,74)$ & $(3,74)$ & $(3,74)$ \\
$\delta_{2}$ & 30,25 & $-19,70$ & $-24,44$ \\
& $(35,23)$ & $(35,23)$ & $(35,23)$ \\
\hline
\end{tabular}

Tabela 4.4 Mudanças (em \%) nas estimativas dos parâmetros dos modelos ajustados aos dados de estoque depois de excluída a observação 22.

\begin{tabular}{c|ccc}
\hline Parâmetro & Normal & $t_{4}$ & Logístico-II \\
\hline$\beta_{0}$ & 4,88 & 4,77 & 4,75 \\
& $(11,57)$ & $(10,52)$ & $(11,30)$ \\
$\beta_{1}$ & $-2,51$ & $-2,73$ & $-2,62$ \\
& $(13,86)$ & $(11,49)$ & $(12,87)$ \\
$\beta_{2}$ & $-0,85$ & $-0,70$ & $-0,67$ \\
& $(2,09)$ & $(2,84)$ & $(2,62)$ \\
$\alpha$ & 2,20 & $-4,30$ & 8,47 \\
& $(2,21)$ & $(2,21)$ & $(2,21)$ \\
$\delta_{2}$ & 7,46 & 8,15 & 8,00 \\
& $(3,47)$ & $(3,47)$ & $(3,47)$ \\
\hline
\end{tabular}




\section{CAPÍTULO 5}

\section{Métodos restritos em modelos simétricos}

\subsection{Introdução}

Nos anos de 1959 e 1961 deram-se as primeiras publicações sobre testes para hipóteses na forma de desigualdades. Esses trabalhos, devidos a Bartholomew (1959a, 1959b, 1961), apresentaram resultados importantes para o caso de ordem simples $\left(\mu_{1} \leqslant \ldots \leqslant \mu_{k}\right)$ em $k$ populações normais independentes. Entretanto, foram Kudô $(1963)$ e Nüesch $(1964,1966)$ que mostraram que a distribuição nula do teste da razão de verossimilhanças para testar hipóteses de igualdades lineares contra desigualdades lineares para os coeficientes de um modelo normal linear, é uma mistura de distribuições do tipo qui-quadrado, ponderadas por pesos, diferindo do caso usual, isto é, o caso em que não há restrições sobre os parâmetros do modelo. Quando a variância das observações é desconhecida, a distribuição nula da estatística da razão de verossimilhanças é uma mistura de distribuições do tipo beta ou $F$. Os pesos para o cálculo dessas distribuições no caso de $k$ populações, sob a hipótese nula, não dependem dos parâmetros. Perlman (1969) mostrou que a distribuição nula do teste da razão de verossimilhanças para testar hipóteses de desigualdades lineares, em que a hipótese nula é composta por desigualdades é, também, uma mistura de qui-quadrados, e propôs um lema no qual define a situação menos favorável quando os pesos não dependem dos parâmetros. Porém, à medida que o número de restrições aumenta, esses pesos tomam formas complexas. Existem formas fechadas para os pesos até quatro restrições. Bohrer e Chow (1978) escreveram um programa computacional para calcular os pesos até 10 restrições. Esse programa faz o uso de integração numérica utilizando o enfoque dado em Childs (1967). No caso de termos uma estrutura de regressão, os pesos geralmente dependem dos parâmetros do modelo sob a hipótese nula. É importante 
notar que como os pesos dependem dos parâmetros, a distribuição nula não é mais única como ocorre no caso de $k$ populações. Em Shapiro $(1985,1988)$ encontra-se um resumo sobre a obtenção desses pesos. Todas as dificuldades em calcular os pesos têm motivado o desenvolvimento de várias linhas de pesquisa nesta área. Naturalmente, os resultados de $k$ populações foram estendidos para a família exponencial e, também, para outras distribuições, tais como a multinomial. Nesses casos, sob condições gerais de regularidade, o teste da razão de verossimilhanças tem distribuição nula assintótica, que é uma mistura de qui-quadrados ponderadas com pesos similares aos do caso normal. Lee, Robertson e Wright (1993) fazem uma revisão das diversas aproximações desenvolvidas para os pesos e apresentam limites mais precisos, que podem ser usados quando os pesos não tomam formas fechadas. Barlow, Bartholomew, Bremmer e Brunk (1972) e Robertson, Wright e Dykstra (1988) e Paula (1997) lançaram livros sobre este assunto, os quais são excelentes referências para leitura.

O estudo do poder para os testes de hipóteses com restrições é feito através de métodos de Monte Carlo ou de aproximações em torno da hipótese nula, uma vez que os pesos assumem expressões bem mais complexas na hipótese alternativa. Somente em alguns casos particulares essas funções têm forma fechada.

Nos últimos 15 anos, o estudo de testes para hipóteses com restrições, com enfoque em regressão, teve um grande número de artigos publicados. Kodde e Palm (1986) estenderam os resultados de Kudô (1963) para o caso de hipóteses nãolineares e propuseram o uso de um teste do tipo Wald que, sob certas condições de regularidade, pode ser aplicado para situações em que a função de verossimilhança é desconhecida. Gouriéroux e Monfort (1995) mostram a equivalência assintótica da estatística do tipo Wald com algumas estatísticas usuais. Wolak (1987,1989a) trata o problema de teste de hipóteses com restrição para o modelo de regressão linear, com estruturas gerais para a matriz de variância-covariância dos erros. Wolak (1987) propõe uma estatística da razão de verossimilhanças modificada quando a matriz de correlação é conhecida, porém as variâncias são desconhecidas, e mostra que a distribuição nula é uma mistura de distribuições do tipo F. Wolak (1989a) 
estende os resultados de Gouriéroux, Holly e Monfort (1982) para o caso da matriz de variância-covariância ser desconhecida e depender de um número finito de parâmetros.

Piegorch (1990) apresenta aplicações desta teoria em modelos lineares generalizados com resposta binária e alguns estudos de simulação, comparando o poder do teste da razão de verossimilhanças com restrições nos parâmetros com o poder de alguns métodos de comparações múltiplas. Silvapulle $(1991,1994)$ estuda a aplicação desse tipo de teste em modelos de regressão com função de verossimilhança côncava, que englobam os modelos lineares generalizados com ligação canônica e os modelos de regressão de Cox. Wolak (1991) demonstra para uma classe ampla de modelos de regressão, que a distribuição nula menos favorável não é, necessariamente, atingida quando todas as restrições são satisfeitas na forma de igualdades. Nesse artigo, ele propõe um lema, estendendo o resultado encontrado por Perlman, no qual define um subconjunto da hipótese nula que contém a situação menos favorável. Para determinar a situação menos favorável deve-se pecorrer todo esse subconjunto, no qual, computacionalmente, pode ser muito dispendioso. Wolak (1989b) sugere o uso de testes locais que, sob condições adicionais de regularidade, possam levar à solução do problema. Farhmeir e Klinger (1994) tratam da estimação e teste em modelos lineares generalizados para hipóteses de restrição em desigualdades lineares, e apresentam estudos numéricos em que propõem um método para a determinação da situação menos favorável definida no lema de Wolak. Paula e Sen (1995) verificam que os pesos envolvidos na distribuição nula assintótica do teste da razão de verossimilhanças, para algumas subclasses de modelos lineares generalizados e para algumas hipóteses com restrição de ordem, não dependem dos parâmetros sob a hipótese nula quando estruturas particulares são assumidas para a matriz modelo. Paula e Rojas (1997) aplicam esta teoria em modelos de regressão com distribuição do valor extremo com parâmetro de dispersão desconhecido. Silvapulle e Silvapulle (1995) propõem um teste, tipo-escore, para testar alternativas unilaterais em modelos de regressão geral, que pode incluir observações correlacionadas. Hipóteses de ordem com medidas repetidas são consideradas, por Shin, Park 
e Park (1996) e Park, Shin e Park (1998) e, mais recentemente, Cardoso-Neto e Paula (2001) examinam a aplicação do teste tipo-Wald proposto por Kodde e Palm (1986) em modelos lineares generalizados com observações correlacionadas. Paula e Artes (2000) utilizam o teste escore, proposto por Silvapulle e Silvapulle (1995), para testar correlação positiva multivariada (superdispersão) em modelos logísticos para os dados agrupados. Recentemente, Verbeke e Molenberghs (2003), também, aplicam o teste de Silvapulle e Silvapulle (1995) para testar presença de efeitos aleatórios em modelos normais marginais. Sen e Silvapulle (2002) apresentam uma excelente revisão na área de modelos restritos.

\subsection{Inferência com restrições em igualdades e desigualdades lineares}

\subsubsection{Igualdades lineares}

Suponha primeiro que estamos interessados em estimar o vetor de parâmetros $\boldsymbol{\beta}$ sob $k$ restrições lineares independentes $\mathbf{C}_{j}^{T} \boldsymbol{\beta}-\mathrm{d}_{j}=0$, em que $\mathbf{C}_{j}, j=1, \ldots, k$, são $p \times 1$ vetores $\mathrm{e}_{j}, j=1, \ldots, k$, são escalares, ambos números conhecidos e fixados. O problema aqui é maximizar o logaritmo da função de verossimilhança $L(\theta)$ sujeito a restrições lineares $\mathbf{C} \boldsymbol{\beta}-\mathbf{d}=0$, em que $\mathbf{C}=\left(\mathbf{C}_{1}^{T}, \ldots, \mathbf{C}_{k}^{T}\right)^{T}$ e $\mathbf{d}=\left(\mathrm{d}_{1}, \ldots, \mathrm{d}_{k}\right)^{T}$. Similar a Nyquist (1991), que investigou este problema em modelos lineares generalizados, aplicaremos a metodologia da função penalizada considerando a função penalizada quadrática (vide, por exemplo, Fiacco e McComick, 1968; Ryan, 1974)

$$
\mathrm{P}(\boldsymbol{\theta}, \boldsymbol{\tau})=\mathrm{L}(\boldsymbol{\theta})-\frac{1}{2} \sum_{j=1}^{k} \tau_{j}\left(\mathrm{~d}_{j}-\mathrm{C}_{j}^{T} \boldsymbol{\beta}\right)^{2} .
$$

O procedimento consiste em encontrar a solução de $\max _{\{\beta, \phi\}} \mathrm{P}(\theta, \tau)$ para valores fixados e positivos de $\tau_{j}, j=1, \ldots, k$. A solução para $\boldsymbol{\beta}$ será denotada por $\boldsymbol{\beta}(\boldsymbol{\tau})$. O estimador restrito de igualdades é dado por

$$
\hat{\boldsymbol{\beta}}^{0}=\lim _{\tau_{1}, \ldots, \tau_{k} \rightarrow \infty} \boldsymbol{\beta}(\tau)
$$

Usando um enfoque similar ao dado em Nyquist (1991) pode-se mostrar que $\beta(\tau)$ é a solução do seguinte processo iterativo : 


$$
\begin{aligned}
\beta^{0(r+1)}= & \left\{\mathrm{X}^{T} \mathrm{D}\left(\mathrm{v}^{(r)}\right) \mathrm{X}\right\}^{-1} \mathrm{X}^{T} \mathrm{D}\left(\mathrm{v}^{(r)}\right) \mathrm{y}+\left\{\mathrm{X}^{T} \mathrm{D}\left(\mathrm{v}^{(r)}\right) \mathrm{X}\right\}^{-1} \mathrm{C}^{T} \times \\
& {\left[\mathrm{C}\left\{\mathrm{X}^{T} \mathrm{D}\left(\mathrm{v}^{(r)}\right) \mathrm{X}\right\}^{-1} \mathrm{C}^{T}\right]^{-1}\left[\mathrm{~d}-\mathrm{C}\left\{\mathrm{X}^{T} \mathrm{D}\left(\mathrm{v}^{(r)}\right) \mathrm{X}\right\}^{-1}\right.} \\
& \left.\mathrm{X}^{T} \mathrm{D}\left(\mathrm{v}^{(r)}\right) \mathrm{y}\right]
\end{aligned}
$$

para $r=0,1, \ldots$, em que $\phi^{(r)}$ é obtido de (2.7). O processo iterativo (5.1) pode ser, alternativamente, expresso na forma

$$
\begin{aligned}
\beta^{0(r+1)}= & \mathrm{b}^{(r+1)}+\left\{\mathrm{X}^{T} \mathrm{D}\left(\mathrm{v}^{(r)}\right) \mathrm{X}\right\}^{-1} \mathrm{C}^{T}\left[\mathrm{C}\left\{\mathrm{X}^{T} \mathrm{D}\left(\mathrm{v}^{(r)}\right) \mathrm{X}\right\}^{-1} \mathrm{C}^{T}\right]^{-1} \\
& \left(\mathrm{~d}-\mathrm{Cb}^{(r+1)}\right)
\end{aligned}
$$

para $r=0,1, \ldots$, em que $\mathrm{b}^{(r+1)}$ denota $\boldsymbol{\beta}^{(r+1)}$ avaliado na estimativa corrente restrita. $\mathrm{b}^{(r+1)}$ pode ser considerada uma estimativa de mínimos quadrados reponderados, sendo dada por $\left\{\mathrm{X}^{T} \mathbf{D}\left(\mathbf{v}^{(r)}\right) \mathrm{X}\right\}^{-1} \mathrm{X}^{T} \mathrm{D}\left(\mathbf{v}^{(r)}\right) \mathrm{y}$ (com os pesos avaliados na estimativa restrita de passos anteriores) mais um termo de correção. O termo adicionado é o produto de dois fatores, uma constante e a diferença entre $\mathrm{d}$ e $\mathrm{Cb}^{(r+1)}$. Desenvolvemos uma macro em S-Plus (Becker, Chambers e Wilk, 1988 e Chambers e Hastie, 1992) e R (Ihaka e Gentleman, 1996) para ajustar modelos simétricos bem como alguns procedimentos de diagnóstico. Essa macro está disponível no endereço www.de.ufpe.br/ cysneiros/elliptical/elliptical.html.

É possível mostrar que sob certas condições de regularidade (veja, por exemplo, Gouriéroux e Monfort, 1995, Seção 10.3) $\hat{\boldsymbol{\beta}}^{0}$ é um estimador consistente de $\boldsymbol{\beta}$, tal que

$$
\sqrt{n}\left(\hat{\boldsymbol{\beta}}^{0}-\boldsymbol{\beta}\right) \stackrel{\mathrm{d}}{\rightarrow} \mathrm{N}_{p}\left(0,\left(\mathbf{J}_{\beta \beta}^{0}\right)^{-1}\right)
$$

sendo

$$
\mathbf{J}_{\beta \beta}^{0}=\lim _{\tau_{1}, \ldots, \tau_{k} \rightarrow \infty}\left[\lim _{n \rightarrow \infty} \frac{1}{n} \mathrm{E}\left\{-\frac{\partial \mathrm{P}(\boldsymbol{\theta}, \boldsymbol{\tau})}{\partial \boldsymbol{\beta} \partial \boldsymbol{\beta}^{T}}\right\}\right]
$$

e

$$
\mathrm{E}\left\{-\frac{\partial \mathrm{P}(\boldsymbol{\theta}, \boldsymbol{\tau})}{\partial \boldsymbol{\beta} \partial \boldsymbol{\beta}^{T}}\right\}=\frac{4 d_{g}}{\phi} \mathrm{X}^{T} \mathrm{X}+\mathrm{C}^{T} \mathrm{D}(\boldsymbol{\tau}) \mathrm{C}
$$


$\operatorname{com} \mathbf{D}(\boldsymbol{\tau})=\operatorname{diag}\left\{\tau_{1}, \ldots, \tau_{k}\right\}$. Então, um estimador consistente da matriz de variância-covariância assintótica de $\hat{\boldsymbol{\beta}}^{0}$ fica dado por

$$
\lim _{\tau_{1}, \ldots, \tau_{k} \rightarrow \infty}\left\{\frac{4 d_{g}}{\phi} \mathbf{X}^{T} \mathbf{X}+\mathbf{C}^{T} \mathbf{D}(\tau) \mathbf{C}\right\}^{-1}=\mathbf{K}_{\beta \beta}^{-1}\left\{\mathrm{I}_{p}-\mathrm{C}^{T}\left(\mathrm{CK}_{\beta \beta}^{-1} \mathrm{C}^{T}\right)^{-1} \mathrm{CK}_{\beta \beta}^{-1}\right\},
$$

o qual pode ser avaliado em alguma estimativa consistente de $\beta$, tais como $\hat{\boldsymbol{\beta}}$ ou $\hat{\beta}^{0}$.

Suponha, agora, as hipóteses $\mathrm{H}_{0}: \mathrm{C} \boldsymbol{\beta}=\mathrm{d}$ contra $\mathrm{H}_{1}: \mathrm{C} \boldsymbol{\beta} \neq \mathrm{d}$. Os métodos mais usuais para testar estas hipóteses lineares são os testes da razão de verossimilhanças, Wald e escore. As estatísticas são dadas aqui, respectivamente, por

$$
\begin{aligned}
\xi_{R V}^{*} & =2\left\{\mathrm{~L}(\hat{\boldsymbol{\beta}}, \hat{\phi})-\mathrm{L}\left(\hat{\boldsymbol{\beta}}^{0}, \hat{\phi}_{0}\right)\right\} \\
& =2\left[\frac{n}{2} \log \left(\frac{\hat{\phi}_{0}}{\hat{\phi}}\right)+\sum_{i=1}^{n} \log \left\{\frac{g\left\{\left(y_{i}-\mathbf{x}_{i}^{T} \hat{\boldsymbol{\beta}}\right)^{2} / \hat{\phi}\right\}}{g\left\{\left(y_{i}-\mathbf{x}_{i}^{T} \hat{\boldsymbol{\beta}}^{0}\right)^{2} / \hat{\phi}_{0}\right\}}\right\}\right] \\
\xi_{W}^{*} & =(\mathbf{C} \hat{\boldsymbol{\beta}}-\mathbf{d})^{T} \hat{\operatorname{Var}}^{-1}(\mathbf{C} \hat{\boldsymbol{\beta}})(\mathbf{C} \hat{\boldsymbol{\beta}}-\mathbf{d}) \\
& =(\mathbf{C} \hat{\boldsymbol{\beta}}-\mathbf{d})^{T}\left(\mathbf{C} \hat{\mathbf{K}}_{\beta \beta}^{-1} \mathbf{C}^{T}\right)^{-1}(\mathbf{C} \hat{\boldsymbol{\beta}}-\mathrm{d}) \\
& =\frac{4 d_{g}}{\hat{\phi}}(\mathbf{C} \hat{\boldsymbol{\beta}}-d)^{T}\left\{\mathbf{C}\left(\mathbf{X}^{T} \mathbf{X}\right)^{-1} \mathbf{C}^{T}\right\}^{-1}(\mathbf{C} \hat{\boldsymbol{\beta}}-d) \mathrm{e} \\
\xi_{S R}^{*} & =\left\{\mathbf{U}_{\beta}\left(\hat{\boldsymbol{\beta}}^{0}, \hat{\phi}_{0}\right)-\mathbf{U}_{\beta}(\hat{\boldsymbol{\beta}}, \hat{\phi})\right\}^{T} \hat{\operatorname{Var}}_{0}(\hat{\boldsymbol{\beta}})\left\{\mathbf{U}_{\beta}\left(\hat{\boldsymbol{\beta}}^{0}, \hat{\phi}_{0}\right)-\mathbf{U}_{\beta}(\hat{\boldsymbol{\beta}}, \hat{\phi})\right\} \\
& =\mathbf{U}_{\beta}\left(\hat{\boldsymbol{\beta}}^{0}, \hat{\phi}_{0}\right)^{T}\left(\hat{\mathbf{K}}_{\beta \beta}^{0}\right)^{-1} \mathbf{U}_{\beta}\left(\hat{\boldsymbol{\beta}}^{0}, \hat{\phi}_{0}^{2}\right) \\
& =\frac{\hat{\phi}_{0}}{4 d_{g}} \mathbf{U}_{\beta}\left(\hat{\boldsymbol{\beta}}^{0}, \hat{\phi}_{0}\right)^{T}\left(\mathbf{X}^{T} \mathbf{X}\right)^{-1} \mathbf{U}_{\beta}\left(\hat{\boldsymbol{\beta}}^{0}, \hat{\phi}_{0}\right)
\end{aligned}
$$

em que $\hat{\mathbf{K}}_{\beta \beta}$ e $\hat{\mathbf{K}}_{\beta \beta}^{0}$ são as matrizes de informação avaliadas em $\left(\hat{\boldsymbol{\beta}}^{T}, \hat{\phi}\right)^{T}$ e $\left(\hat{\boldsymbol{\beta}}^{0}{ }^{T}, \hat{\phi}_{0}\right)^{T}$, respectivamente. Sob $\mathrm{H}_{0}$ e para $n$ grande, temos que $\xi_{R V}^{*}, \xi_{W}^{*}$ e $\xi_{S R}^{*}$ têm distribuição qui-quadrado central com $k$ graus de liberdade.

\subsubsection{Desigualdades lineares}

Suponha, agora, que estamos interessados em obter a estimativa de máxima verossimilhança de $\beta$ no modelo de regressão linear simétrico sujeito às restrições $\mathrm{C} \beta-\mathrm{d} \geqslant 0$; isto é, temos interesse em resolver o problema $\max _{\{(\mathrm{C} \beta-\mathrm{d} \geqslant 0), \phi\}} \mathrm{L}(\boldsymbol{\beta}, \phi)$. 
Podemos aplicar as condições de Kuhn-Tucker (vide, por exemplo, Hildreth, 1957; Luenberger 1969; Nocedal e Wright, 1999) para atingir o máximo global restrito. Considere, então, a função Lagrangiana

$$
\mathcal{L}(\boldsymbol{\beta}, \phi)=\mathrm{L}(\boldsymbol{\beta}, \phi)+\sum_{i=1}^{k} \lambda_{j}\left(\mathrm{C}_{j}^{T} \boldsymbol{\beta}-\mathrm{d}_{j}\right),
$$

em que $\lambda=\left(\lambda_{1}, \ldots, \lambda_{k}\right)^{T} \geqslant 0$ denota o vetor de multiplicadores de Lagrange. As condições suficientes para garantir que $\tilde{\boldsymbol{\beta}}$ corresponda à estimativa restrita por desigualdades (veja, por exemplo, Fahrmeir e Klinger, 1994) são dadas por

(i) $\mathrm{C}_{j}^{T} \tilde{\boldsymbol{\beta}}-\mathrm{d}_{j}=0$ para $j \in \mathbf{I} \subseteq\{1, \ldots, k\}$ e $\mathbf{C}_{j}^{T} \tilde{\boldsymbol{\beta}}>\mathrm{d}_{j}$ para todo $j \notin \mathbf{I}$; isto é, $\tilde{\boldsymbol{\beta}}$ é um ponto admissível;

(ii) existe $\tilde{\lambda}=\left(\tilde{\lambda}_{1}, \ldots, \tilde{\lambda}_{k}\right)^{T} \geqslant 0$ tal que $\partial \mathcal{L}(\boldsymbol{\beta}, \phi) /\left.\partial \boldsymbol{\beta}\right|_{\left(\tilde{\boldsymbol{\beta}}^{T}, \tilde{\phi}\right)^{T}}=0$; isto é, $\left(\tilde{\boldsymbol{\beta}}^{T}, \tilde{\phi}\right)^{T}$ é um ponto estacionário;

(iii) $\left.\mathrm{s}^{T} \ddot{\mathrm{L}}_{\beta \beta} \mathrm{s}\right|_{\left(\tilde{\beta}^{T}, \tilde{\phi}\right)^{T}}<0$ para todo $\mathrm{s} \neq 0$ e $\mathrm{s} \in\left\{\mathrm{s} \mid \mathbf{C}_{j}^{T} \mathrm{~s}-\mathrm{d}_{j}=0, j \in \mathbf{I}, \tilde{\lambda}_{j}>\right.$ 0 e $\left.\mathrm{C}_{j}^{T} \mathrm{~s}-\mathrm{d}_{j}>0, j \notin \mathrm{I}, \tilde{\lambda}_{j}=0\right\}$.

Essas condições são equivalentes a encontrar $\tilde{\beta}$ através de um procedimento de busca que consiste em maximizar $\mathrm{L}(\boldsymbol{\beta}, \phi)$ sujeito a $\mathrm{C}_{j}^{T} \boldsymbol{\beta}-\mathrm{d}_{j}=0, j \in \mathbf{I}$, para cada $I \subseteq\{1, \ldots, k\}$. A estimativa restrita por desigualdades $\tilde{\beta}$ é obtida através de problemas de maximização que satisfaçam as condições (i), (ii) e (iii). Então, o problema restrito por desigualdades reduz-se a um problema restrito por igualdades, que pode ser resolvido pelos procedimentos dados na Seção 5.2.1.

A distribuição assintótica de $\tilde{\boldsymbol{\beta}}$, supondo que o espaço paramétrico é da forma $\{\mathrm{C} \boldsymbol{\beta} \geqslant \mathrm{d}\}$, não é necessariamente normal. Essa distribuição depende se o verdadeiro valor do parâmetro satisfaz $\mathrm{C} \boldsymbol{\beta}-\mathrm{d}>0$ ou $\mathrm{C} \boldsymbol{\beta}-\mathrm{d}=\mathbf{0}$. Para o primeiro caso o estimador restrito por desigualdades coincide assintoticamente com o estimador irrestrito e, portanto, $\tilde{\boldsymbol{\beta}}$ tem a mesma distribuição assintótica de $\hat{\boldsymbol{\beta}}$. Contudo, se o verdadeiro valor pertence à fronteira do conjunto de desigualdades lineares, a distribuição assintótica de $\tilde{\boldsymbol{\beta}}$ tem a forma de uma normal truncada em $\mathbf{C} \boldsymbol{\beta}-\mathrm{d}=\mathbf{0}$ (veja discussão, por exemplo, em Gouriéroux e Monfort, 1995, Seção 21.1). 


\subsection{Testes unilaterais}

\subsubsection{Caso 1}

Consideraremos nesta seção o problema de testar as hipóteses $\mathrm{H}_{0}: \mathrm{C} \beta=\mathrm{d}$ contra $\mathrm{H}_{2}: \mathrm{C} \beta \geqslant \mathrm{d}$, com pelo menos uma desigualdade estrita em $\mathrm{H}_{2}$. Esse tipo de hipótese unilateral não exige, necessariamente, que o espaço paramétrico seja do tipo $\{\mathbf{C} \boldsymbol{\beta} \geqslant \mathbf{d}\}$. Em geral, essas hipóteses são testadas quando existem suspeitas de que os parâmetros satisfazem determinadas restrições, embora o contrário seja possível teoricamente. As estatísticas usuais razão de verossimilhanças, Wald e escore tomam, neste caso, as formas

$$
\begin{aligned}
\xi_{R V} & =2\left[\mathrm{~L}(\tilde{\boldsymbol{\beta}}, \tilde{\phi})-\mathrm{L}\left(\hat{\boldsymbol{\beta}}^{0}, \hat{\phi}_{0}\right)\right] \\
& =2\left[\frac{n}{2} \log \left(\frac{\hat{\phi}_{0}}{\tilde{\phi}}\right)+\sum_{i=1}^{n} \log \left\{\frac{g\left\{\left(y_{i}-\mathbf{x}_{i}^{T} \tilde{\boldsymbol{\beta}}\right)^{2} / \tilde{\phi}\right\}}{g\left\{\left(y_{i}-\mathbf{x}_{i}^{T} \hat{\boldsymbol{\beta}}^{0}\right)^{2} / \hat{\phi}_{0}\right\}}\right\}\right], \\
\xi_{W} & =(\mathbf{C} \tilde{\boldsymbol{\beta}}-\mathbf{d})^{T}\left\{\mathbf{C} \tilde{\mathbf{K}}_{\beta \beta}^{-1} \mathbf{C}^{T}\right\}^{-1}(\mathbf{C} \tilde{\boldsymbol{\beta}}-\mathbf{d}) \\
& =\frac{4 d_{g}}{\tilde{\phi}}(\mathbf{C} \tilde{\boldsymbol{\beta}}-\mathbf{d})^{T}\left\{\mathbf{C}\left(\mathbf{X}^{T} \mathbf{X}\right)^{-1} \mathbf{C}^{T}\right\}^{-1}(\mathbf{C} \tilde{\boldsymbol{\beta}}-\mathbf{d}) \mathrm{e} \\
\xi_{S R} & =\left\{\mathbf{U}_{\boldsymbol{\beta}}\left(\hat{\boldsymbol{\beta}}^{0}, \hat{\phi}_{0}\right)-\mathbf{U}_{\beta}(\tilde{\boldsymbol{\beta}}, \tilde{\phi})\right\}^{T}\left(\hat{\mathbf{K}}_{\beta \beta}^{0}\right)^{-1}\left\{\mathbf{U}_{\beta}\left(\hat{\boldsymbol{\beta}}^{0}, \hat{\phi}_{0}\right)-\mathbf{U}_{\beta}(\tilde{\boldsymbol{\beta}}, \tilde{\phi})\right\} \\
& =\frac{\hat{\phi}_{0}}{4 d_{g}}\left\{\mathbf{U}_{\beta}\left(\hat{\boldsymbol{\beta}}^{0}, \hat{\phi}_{0}\right)-\mathbf{U}_{\beta}(\tilde{\boldsymbol{\beta}}, \tilde{\phi})\right\}^{T}\left(\mathbf{X}^{T} \mathbf{X}\right)^{-1}\left\{\mathbf{U}_{\beta}\left(\hat{\boldsymbol{\beta}}^{0}, \hat{\phi}_{0}\right)-\mathbf{U}_{\beta}(\tilde{\boldsymbol{\beta}}, \tilde{\phi})\right\},
\end{aligned}
$$

respectivamente. Sob condições regulares dadas em Gouriéroux e Monfort (1995, Seção 21.3) por exemplo que o espaço paramétrico de $\beta$ é aberto, segue-se que as estatísticas $\xi_{R V}, \xi_{W}$ e $\xi_{S R}$ são assintoticamente equivalentes a uma mistura de distribuições qui-quadrados, tais que

$$
\operatorname{Pr}\left\{\xi_{R V} \geqslant c\right\}=\sum_{\ell=0}^{k} \omega(k, \ell ; \Delta) \operatorname{Pr}\left\{\chi_{\ell}^{2} \geqslant c\right\},
$$

em que $c \geqslant 0, \Delta=\mathrm{CK}_{\beta \beta}^{-1} \mathrm{C}^{T}, \chi_{0}^{2}$ denota a distribuição degenerada na origem e $\omega(k, \ell ; \Delta)$ 's são conhecidos como probabilidades de nível (veja definição e expressões, por exemplo, em Shapiro, 1985) as quais são expressas como funções dos coeficientes de correlação associados com a matriz $\Delta$. Esses coeficientes de correlação são o mínimo de informação necessária para calcular a distribuição nula 
assintótica dada em (5.3) uma vez que $\omega(k, \ell ; \Delta)$ depende de $\Delta$ somente através da matriz de correlação. Devido à dificuldade de cálculo das probabilidades de nível para cinco ou mais restrições, várias aproximações têm sido propostas (veja, por exemplo, Robertson, Wright e Dykstra 1988, Cap. 3). Todavia, procedimentos computacionais para o cálculo dos pesos $w(k, \ell ; \Delta)$ estão disponíveis (veja, por exemplo, Bohrer e Chow, 1978 e Sun, 1988a, 1988b). Se os pesos $\omega(k, \ell ; \Delta)$ 's não dependerem de $\beta$ através dos coeficientes da matriz de correlação associada à $\Delta$, então, a distribuição dada em (5.3) é única. Examinando a expressão de $K_{\beta \beta}$ dada na Seção 5.2 , podemos concluir que $\omega(k, \ell ; \boldsymbol{\Delta})$ não depende de $\boldsymbol{\beta}$ na classe de modelos lineares simétricos. Essa propriedade não é verificada em geral. Por exemplo, em modelos lineares generalizados ocorre somente em alguns casos particulares (veja, por exemplo, Paula e Sen, 1994,1995 e Paula, 1996, 1999a).

\subsubsection{Caso 2}

Consideraremos agora as hipóteses $\mathrm{H}_{2}: \mathbf{C} \boldsymbol{\beta} \geqslant \mathbf{d}$ contra $\mathrm{H}_{1}-\mathrm{H}_{2}$. Neste caso, as estatísticas usuais razão de verosssimilhanças, Wald e escore tomam a forma

$$
\begin{aligned}
\xi_{R V}^{c} & =2\{\mathrm{~L}(\hat{\boldsymbol{\beta}}, \hat{\phi})-\mathrm{L}(\tilde{\boldsymbol{\beta}}, \tilde{\phi})\} \\
& =2\left[\frac{n}{2} \log \left(\frac{\tilde{\phi}}{\hat{\phi}}\right)+\sum_{i=1}^{n} \log \left\{\frac{g\left\{\left(y_{i}-\mathbf{x}_{i}^{T} \hat{\boldsymbol{\beta}}\right)^{2} / \hat{\phi}\right\}}{g\left\{\left(y_{i}-\mathbf{x}_{i}^{T} \tilde{\boldsymbol{\beta}}\right)^{2} / \tilde{\phi}\right\}}\right\}\right], \\
\xi_{W}^{c} & =(\mathbf{C} \hat{\boldsymbol{\beta}}-\mathbf{C} \tilde{\boldsymbol{\beta}})^{T}\left\{\mathbf{C} \tilde{\mathbf{K}}_{\beta \beta}^{-1} \mathbf{C}^{T}\right\}^{-1}(\mathbf{C} \hat{\boldsymbol{\beta}}-\mathbf{C} \tilde{\boldsymbol{\beta}}) \\
& =\frac{4 d_{g}}{\hat{\phi}}(\mathbf{C} \hat{\boldsymbol{\beta}}-\mathbf{C} \tilde{\boldsymbol{\beta}})^{T}\left\{\mathbf{C}\left(\mathbf{X}^{T} \mathbf{X}\right)^{-1} \mathbf{C}^{T}\right\}^{-1}(\mathbf{C} \hat{\boldsymbol{\beta}}-\mathbf{C} \tilde{\boldsymbol{\beta}}) \mathrm{e} \\
\xi_{S R}^{c} & =\mathbf{U}_{\beta}(\tilde{\boldsymbol{\beta}}, \tilde{\phi})^{T}\left(\hat{\mathbf{K}}_{\beta \beta}^{0}\right)^{-1} \mathbf{U}_{\beta}(\tilde{\boldsymbol{\beta}}, \tilde{\phi}) \\
& =\frac{\tilde{\phi}}{4 d_{g}} \mathbf{U}_{\beta}(\tilde{\boldsymbol{\beta}}, \tilde{\phi})^{T}\left(\mathbf{X}^{T} \mathbf{X}\right)^{-1} \mathbf{U}_{\beta}(\tilde{\boldsymbol{\beta}}, \tilde{\phi}) .
\end{aligned}
$$

Quando os pesos $\omega(k, \ell ; \Delta)$ 's dependem de $\beta$ a distribuição nula assintótica menos favorável das estatísticas dos testes pode ser muito complicada de ser determinada (vide, Wolak, 1991). Felizmente, como foi observado na última seção, $\omega(k, \ell ; \Delta)$ não depende de $\beta$ na classe de modelos lineares simétricos. Assim, a 
distribuição nula menos favorável das estatísticas $\xi_{R V}^{c}, \xi_{W}^{c}$ e $\xi_{S R}^{c}$ fica dada por

$$
\operatorname{Pr}\left\{\xi_{R V}^{c} \geqslant c\right\}=\sum_{\ell=0}^{k} \omega(k, k-\ell ; \Delta) \operatorname{Pr}\left\{\chi_{\ell}^{2} \geqslant c\right\},
$$

em que $c \geqslant 0$. Um algoritmo de busca é necessário para encontrar a distribuição nula menos favorável quando os pesos dependem de $\boldsymbol{\beta}$ (vide, Wolak, 1991).

\subsection{Assinaturas de TV a cabo}

Nesta seção será reanalizado um exemplo discutido em Ramanathan (1993), (vide também Cysneiros e Paula, 2003) em que sete variáveis são observadas em 40 áreas metropolitanas (veja Apêndice E). O principal interesse é explicar o número (em milhares) de assinantes de TV a cabo, (y) segundo o número (em milhares) de domicílios na área, $\left(x_{1}\right)$, a renda per capita por domicílio com TV a cabo, $\left(x_{2}\right)$, a taxa de instalação, $\left(x_{3}\right)$, o custo médio mensal de manutenção, $\left(x_{4}\right)$, o número de canais a cabo disponíveis na área, $\left(x_{5}\right)$ e o número de canais abertos com sinal de boa qualidade na área, $\left(x_{6}\right)$. Como $y$ corresponde a dados de contagem usaremos a transformação raiz quadrada a fim de tentar estabilizar a variância. Propomos, então, o modelo

$$
\sqrt{y_{i}}=\beta_{0}+\sum_{j=1}^{6} \beta_{j} x_{j i}+\epsilon_{i}, \quad i=1, \ldots, 40,
$$

em que $\epsilon_{i} \sim S(0, \phi)$ são erros mutuamente independentes.

Além disso, é razoável esperar que o efeito de cada coeficiente seja unidirecional, embora a direção oposta não seja teoricamente impossível. Por exemplo, é razoável querer testar se o número esperado de assinantes de TV a cabo decresce a medida que o custo médio mensal de manutenção cresce, isto é, testar $H_{0}: \beta_{4}=0$ contra $\mathrm{H}_{2}: \beta_{4}<0$. Seguindo o mesmo raciocínio para as demais variáveis podemos estar interessados em avaliar as direções $\beta_{1} \geqslant 0, \beta_{2} \geqslant 0, \beta_{3} \leqslant 0, \beta_{5} \geqslant 0$ e $\beta_{6} \leqslant 0$.

Análise sob erros normais

Inicialmente, ajustamos o modelo (5.5) assumindo erros normais. As estimativas restritas são dadas na primeira coluna da Tabela 5.1. Aplicando testes $t$-unilaterais 
não rejeitamos a hipótese de que cada coeficiente $\beta_{2}, \beta_{3}$ e $\beta_{4}$ seja igual a zero, ao nível de significância de $5 \%$, enquanto alguma dúvida aparece para o coeficiente $\beta_{5}$ cujo $p$-valor está em torno de $3 \%$. Os coeficientes restantes $\beta_{1}$ e $\beta_{6}$ são altamente significativos nas direções unilaterais. O único coeficiente estimado com sinal oposto é $\hat{\beta}_{3}$, mas isto não é devido a multicolinearidade que é desprezível neste exemplo.

Tabela 5.1 Estimativas de máxima verossimilhança irrestritas (erros padrões).

\begin{tabular}{c|cccc}
\hline Parâmetro & Normal & $t_{6}$ & $\mathrm{PE}(0,3)$ & Logístico-II \\
\hline$\beta_{0}$ & 2,319 & 3,335 & 2,635 & 3,122 \\
& $(2,233)$ & $(1,866)$ & $(1,939)$ & $(1,907)$ \\
$\beta_{1}$ & 0,034 & 0,035 & 0,034 & 0,034 \\
& $(0,002)$ & $(0,002)$ & $(0,002)$ & $(0,002)$ \\
$\beta_{2}$ & 0,0002 & 0,0001 & 0,0002 & 0,0001 \\
& $(0,0003)$ & $(0,0002)$ & $(0,0002)$ & $(0,0002)$ \\
$\beta_{3}$ & 0,035 & 0,010 & 0,023 & 0,014 \\
& $(0,040)$ & $(0,033)$ & $(0,034)$ & $(0,034)$ \\
$\beta_{4}$ & $-0,245$ & $-0,318$ & $-0,268$ & $-0,301$ \\
& $(0,182)$ & $(0,152)$ & $(0,158)$ & $(0,155)$ \\
$\beta_{5}$ & 0,134 & 0,118 & 0,122 & 0,119 \\
& $(0,059)$ & $(0,049)$ & $(0,052)$ & $(0,051)$ \\
$\beta_{6}$ & $-0,361$ & $-0,319$ & $-0,335$ & $-0,327$ \\
& $(0,134)$ & $(0,111)$ & $(0,116)$ & $(0,114)$ \\
$\phi$ & 1,015 & 0,665 & 0,573 & 0,298 \\
& $(0,227)$ & $(0,182)$ & $(0,146)$ & $(0,078)$ \\
\hline \multirow{3}{*}{} & & & &
\end{tabular}


Tabela 5.2 Estimativas de máxima verossimilhança restritas (erros padrões).

\begin{tabular}{c|cccc}
\hline Parâmetro & Normal & $t_{6}$ & $\mathrm{PE}(0,3)$ & Logístico-II \\
\hline$\beta_{0}$ & 2,292 & 3,413 & 2,559 & 3,219 \\
& $(2,205)$ & $(1,860)$ & $(1,948)$ & $(1,906)$ \\
$\beta_{1}$ & 0,034 & 0,035 & 0,035 & 0,035 \\
& $(0,003)$ & $(0,002)$ & $(0,002)$ & $(0,002)$ \\
$\beta_{2}$ & 0,0002 & 0,0001 & 0,0002 & 0,0002 \\
& $(0,0002)$ & $(0,0002)$ & $(0,0002)$ & $(0,0002)$ \\
$\beta_{3}$ & 0,000 & 0,000 & 0,000 & 0,000 \\
& $(0,000)$ & $(0,000)$ & $(0,000)$ & $(0,000)$ \\
$\beta_{4}$ & $-0,208$ & $-0,312$ & $-0,242$ & $-0,292$ \\
& $(0,163)$ & $(0,148)$ & $(0,155)$ & $(0,151)$ \\
$\beta_{5}$ & 0,124 & 0,116 & 0,120 & 0,116 \\
& $(0,054)$ & $(0,049)$ & $(0,051)$ & $(0,050)$ \\
$\beta_{6}$ & $-0,376$ & $-0,321$ & $-0,352$ & $-0,329$ \\
& $(0,122)$ & $(0,111)$ & $(0,116)$ & $(0,113)$ \\
$\phi$ & 1,038 & 0,664 & 0,570 & 0,299 \\
& $(0,232)$ & $(0,182)$ & $(0,148)$ & $(0,079)$ \\
\hline \multirow{4}{*}{${ }_{*}^{*}$} & & & & \\
\hline
\end{tabular}

Então, para verificar se os quatro coeficientes são conjuntamente iguais a zero, aplicamos os testes estatísticos definidos nas Seções 5.2 e 5.3 para analisar, respectivamente, as hipóteses $\mathrm{H}_{0}: \mathbf{C} \boldsymbol{\beta}=0$ contra $\mathrm{H}_{1}: \mathbf{C} \boldsymbol{\beta} \neq 0$ e $\mathrm{H}_{0}: \mathbf{C} \boldsymbol{\beta}=0$ contra $\mathrm{H}_{2}: \mathbf{C} \boldsymbol{\beta} \geqslant 0$, com pelo menos uma desigualdade estrita em $\mathrm{H}_{2}$, em que

$$
\mathbf{C}=\left[\begin{array}{rrrrrrr}
0 & 0 & 1 & 0 & 0 & 0 & 0 \\
0 & 0 & 0 & -1 & 0 & 0 & 0 \\
0 & 0 & 0 & 0 & -1 & 0 & 0 \\
0 & 0 & 0 & 0 & 0 & 1 & 0
\end{array}\right]
$$


Tabela 5.3 Valores das estatísticas dos testes e p-valor (entre parênteses).

\begin{tabular}{c|cccc}
\hline Estatística & Normal & $t_{6}$ & $\mathrm{PE}(0,3)$ & Logístico-II \\
\hline$\xi_{S R}^{*}$ & 6,615 & 8,536 & 9,111 & 8,502 \\
& $(0,158)$ & $(0,074)$ & $(0,058)$ & $(0,075)$ \\
$\xi_{R V}^{*}$ & 7,231 & 8,651 & 8,327 & 8,322 \\
& $(0,124)$ & $(0,070)$ & $(0,080)$ & $(0,080)$ \\
$\xi_{W}^{*}$ & 7,926 & 8,875 & 7,700 & 8,215 \\
& $(0,094)$ & $(0,064)$ & $(0,103)$ & $(0,084)$ \\
$\xi_{S R}$ & 5,875 & 8,387 & 8,665 & 8,262 \\
& $(0,064)$ & $(0,020)$ & $(0,018)$ & $(0,021)$ \\
$\xi_{R V}$ & 6,328 & 8,560 & 7,943 & 8,144 \\
& $(0,052)$ & $(0,019)$ & $(0,025)$ & $(0,023)$ \\
$\xi_{W}$ & 6,856 & 8,901 & 7,546 & 8,146 \\
& $(0,041)$ & $(0,016)$ & $(0,030)$ & $(0,023)$ \\
\hline
\end{tabular}

Os resultados dos testes estatísticos ( $p$-valor entre parênteses) são apresentados na primeira coluna da Tabela 5.3. Pode ser mostrado que a distribuição nula assintótica das estatísticas $\xi_{R V}, \xi_{S R}$ e $\xi_{W}$ é uma mistura de distribuições quiquadrados com pesos $\omega(0,4 ; \Delta)=0,071 ; \omega(1,4 ; \Delta)=0,261 ; \omega(2,4 ; \Delta)=0,373$; $\omega(3,4 ; \Delta)=0,239$ e $\omega(4,4 ; \Delta)=0,056$. Os resultados indicam que a hipótese nula não é rejeitada em geral, ao nível de $10 \%$ para os testes bilaterais e ao nível de $5 \%$ para os testes unilaterais.

Contudo, devido à falta de robustez das estimativas de mínimos quadrados contra observações aberrantes, iniciamos algumas análises de resíduos. A Figura 5.1a apresenta o gráfico dos resíduos padronizados, contra os valores ajustados. O gráfico não nos fornece indícios de alguma tendência sistemática, confirmando a propriedade de que $\sqrt{y}$ estabilizaria a variância dos erros. Além disso, a área 14 aparece com um alto valor residual (maior do que 3), sugerindo a possibilidade da influência dessa observação na decisão da estatística do teste. O envelope gerado, como pro- 
posto por Atkinson (1981), é apresentado na Figura 5.2a indicando que a suposição de distribuição normal para os erros não parece ser inapropriada, mesmo com a área 14 aparecendo fora do envelope.

Tabela 5.4 Valor das estatísticas dos testes e p-valor (entre parênteses) excluindo a área 14.

\begin{tabular}{c|cccc}
\hline Estatística & Normal & $t_{6}$ & $\mathrm{PE}(0,3)$ & Logístico-II \\
\hline$\xi_{S R}^{*}$ & 10,902 & 11,144 & 11,733 & 11,379 \\
& $(0,028)$ & $(0,025)$ & $(0,019)$ & $(0,023)$ \\
$\xi_{R V}^{*}$ & 12,787 & 11,420 & 12,419 & 11,675 \\
& $(0,012)$ & $(0,022)$ & $(0,014)$ & $(0,020)$ \\
$\xi_{W}^{*}$ & 15,133 & 11,865 & 13,971 & 12,209 \\
& $(0,004)$ & $(0,018)$ & $(0,007)$ & $(0,016)$ \\
$\xi_{S R}$ & 10,902 & 11,145 & 11,734 & 11,379 \\
& $(0,006)$ & $(0,006)$ & $(0,004)$ & $(0,005)$ \\
$\xi_{R V}$ & 12,787 & 11,420 & 12,419 & 11,675 \\
& $(0,003)$ & $(0,005)$ & $(0,003)$ & $(0,004)$ \\
$\xi_{W}$ & 15,133 & 11,865 & 13,971 & 12,209 \\
& $(0,001)$ & $(0,004)$ & $(0,001)$ & $(0,003)$ \\
\hline
\end{tabular}

A eliminação dessa área do conjunto de dados reduz drasticamente o $p$-valor das estatísticas dos testes, como podemos notar pela primeira coluna da Tabela 5.4 quando comparado com a Tabela 5.3. Nota-se que, a área 14 tem uma alta proporção de domicílios com TV a cabo, taxa de instalação alta e um custo mensal relativamente alto de manutenção, os quais estão em desacordo com o alto número de assinantes. Calculamos a influência local total $C_{i}$ (Lesaffre e Verbeke, 1998) usando as expressões de curvatura calculadas por Galea, Paula e Uribe-Opazo (2003) em modelos lineares simétricos. Os gráficos de índices de $\mathrm{C}_{i}$ são apresentado nas Figuras 5.3a-5.5a para o caso normal. Como podemos observar nestas figuras, as áreas 14 e 1 aparecem como as observações mais influentes. A eliminação da área 1 faz com que todas as estatísticas dos testes sejam não significantes (veja a primeira 
coluna da Tabela 5.5). Essa área tem uma pequena proporção de domicílios com TV a cabo, porém um grande número de canais a cabo.

Tabela 5.5 Valor das estatísticas dos testes e p-valor (entre parênteses) excluindo a área 1.

\begin{tabular}{c|cccc}
\hline Estatística & Normal & $t_{6}$ & $\mathrm{PE}(0,3)$ & Logístico-II \\
\hline$\xi_{S R}^{*}$ & 3,097 & 6,387 & 5,524 & 5,895 \\
& $(0,542)$ & $(0,172)$ & $(0,238)$ & $(0,207)$ \\
$\xi_{R V}^{*}$ & 3,227 & 6,397 & 5,017 & 5,661 \\
& $(0,521)$ & $(0,171)$ & $(0,286)$ & $(0,226)$ \\
$\xi_{W}^{*}$ & 3,364 & 6,779 & 4,393 & 5,562 \\
& $(0,499)$ & $(0,148)$ & $(0,355)$ & $(0,234)$ \\
$\xi_{S R}$ & 2,841 & 6,387 & 5,482 & 5,895 \\
& $(0,245)$ & $(0,050)$ & $(0,075)$ & $(0,063)$ \\
$\xi_{R V}$ & 2,948 & 6,397 & 5,002 & 5,661 \\
& $(0,234)$ & $(0,050)$ & $(0,094)$ & $(0,070)$ \\
$\xi_{W}$ & 3,062 & 6,799 & 4,449 & 5,562 \\
& $(0,222)$ & $(0,042)$ & $(0,120)$ & $(0,073)$ \\
\hline
\end{tabular}

\section{Análise sob outros erros simétricos}

A fim de tentar acomodar as áreas 1 e 14 ou pelo menos tentar reduzir suas influências nos resultados das estatísticas dos testes, reajustamos o modelo (5.5) assumindo distribuições para os erros com caudas mais pesadas que a normal. Inicialmente, ajustamos o modelo com erros seguindo distribuição $t$-Student $\operatorname{com} \nu$ graus de liberdade. Se assumirmos que $\nu>4$, então um estimativa consistente para $\nu$ pode ser obtida dos resíduos ordinários $r_{i}=y_{i}-\hat{y}_{i}, i=1, \ldots, n$. Essa estimativa é dada por $\hat{\nu}=\frac{4 \hat{m}_{2,1}-6}{4 \hat{m}_{2,1}-3}$, em que $\hat{m}_{2,1}=\frac{1 / n \sum_{i=1}^{n} r_{i}^{4}}{\left(1 / n \sum_{i=1}^{n} r_{i}^{2}\right)^{2}}$ (Arrelano-Valle, 1994). Para o conjunto de dados do exemplo encontramos $\hat{\nu} \approx 6$. As estimativas irrestritas de máxima verossimilhança para os parâmetros sob distribuição $t$-Student $\operatorname{com} \nu=6$ graus de liberdade, são dadas na segunda coluna da Tabela 5.1. Note que os erros 
padrões aproximados sob o modelo $t$-Student são, em geral, menores do que os erros padrões sob o modelo normal. Os valores das estatísticas dos testes bilaterais e unilaterais, são descritos na segunda coluna da Tabela 5.3, indicando pela rejeição da hipótese nula aos níveis de significância de $10 \%$ e 5\%, respectivamente. A análise residual sob o modelo $t$-Student é descrita nas Figuras $5.1 \mathrm{~b}$ e $5.2 \mathrm{~b}$, indicando a área 14 com um alto resíduo, que aqui é acomodado no envelope. O envelope gerado para o modelo $t$-Student não apresenta nenhum aspecto incomum. Eliminando a área 14 nota-se que os valores das estatísticas dos testes, descritos na segunda coluna da Tabela 5.4, não mudam como no caso normal confirmando a robustez da distribuição $t$-Student contra observações aberrantes. As Figuras 5.3b-5.5b apresentam os gráficos de índices de $C_{i}$ para o modelo $t$-Student e, como podemos observar nesta figura, que as áreas 1 e 21 aparecem com alguma influência. A eliminação da área 1 (veja Tabela 5.5) muda a decisão baseada nos testes bilaterais, mas não muda muito os $p$-valores nos testes unilaterais.

Outras duas distribuições para o erro com caudas mais pesadas do que a normal foram também assumidas, exponencial potência com $k=0,3$ e logística-II. Foi assumido arbitrariamente $k=0,3$ para tentar acomodar a observação aberrante 14. As estimativas de máxima verossimilhança irrestritas para os parâmetros dos modelos exponencial potência e logístico-II são, respectivamente, dadas na terceira e quarta colunas da Tabela 5.1. Os valores das estatísticas dos testes são apresentados na terceira e quarta colunas da Tabela 5.3. Como se pode notar, há uma similaridade entre os resultados para esses dois modelos e o modelo $t$-Student com $\nu=6$ graus de liberdade. Pelas Figuras 5.1c, 5.1d, 5.2c e 5.2d, pode-se observar que a área 14, também, aparece como uma observação discrepante, porém, os envelopes gerados apresentam um comportamento similar ao do modelo $t$-Student. Os gráficos de índices de $\mathrm{C}_{i}$ (vide Figuras 5.3c-5.5c e 5.3d-5.5d) confirmam a influência das áreas 1 e 14. A eliminação da área 1 muda mais os p-valores das estatísticas dos testes sob o modelo exponencial potência, no sentido de não rejeitar a hipótese nula, do que sob os modelos $t$-Student e logístico-II. O modelo $t$-Student parece ser o modelo mais robusto dentre todos os modelos ajustados. 
Figura 5.1 Gráfico de $t_{r_{i}}$ contra os valores ajustados para o modelo (5.5) sob erros (a) normal, (b) t-Student com 6 g.l., (c) EP(0,3) e (d) logístico-II.

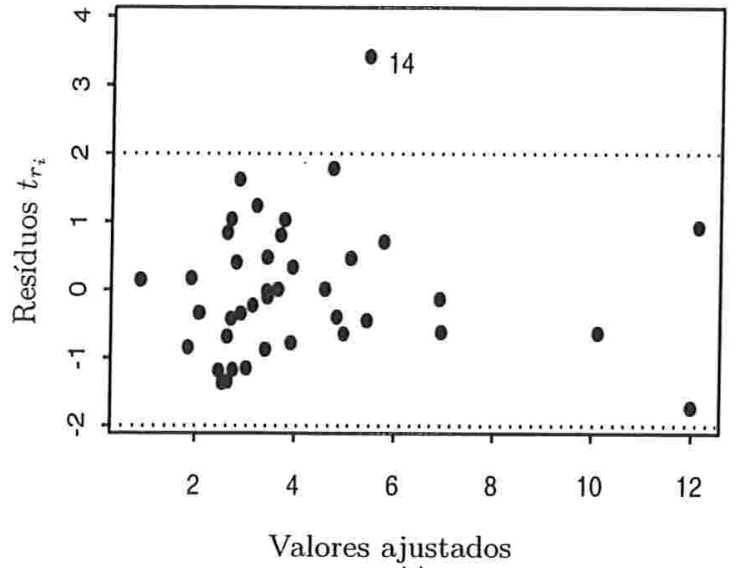

(a)

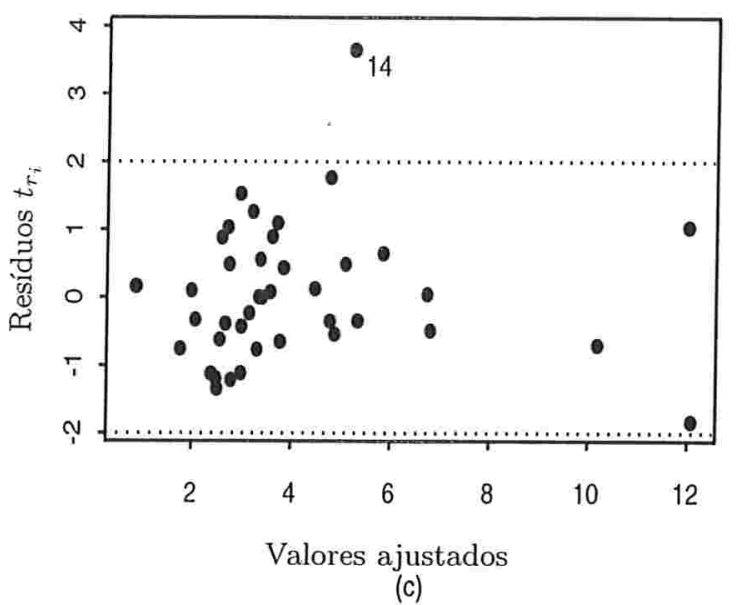

(c)

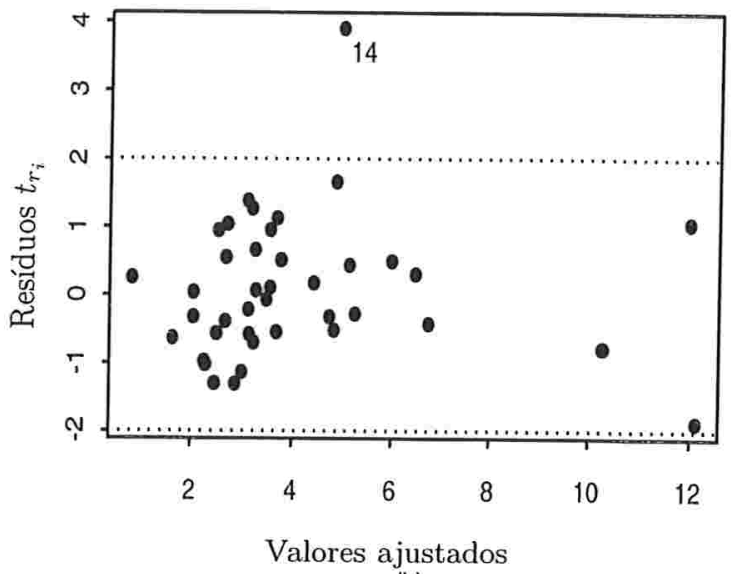

(b)

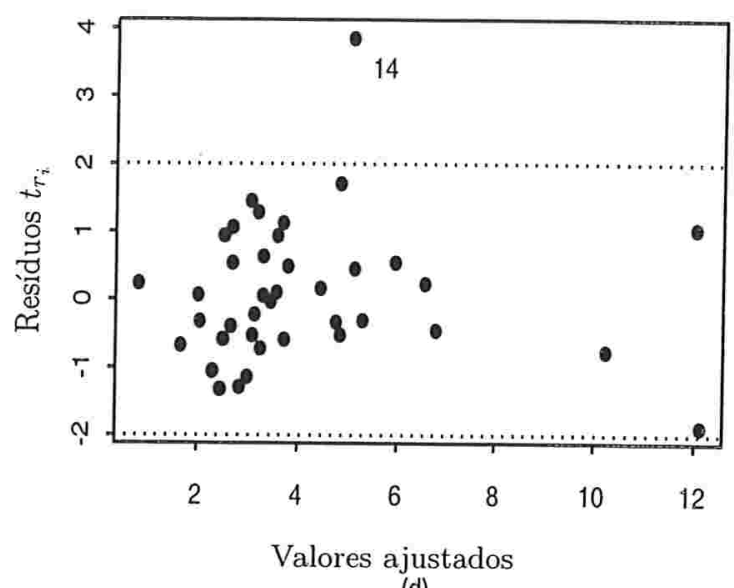

(d) 
Figura 5.2 Gráfico normal de probabilidades com envelope para o resíduo $t_{r_{i}}$ para o modelo (5.5) sob erro (a) normal, (b) t-Student com 6 g.l., (c) $\operatorname{EP}(0,3)$ e (d) logístico-II.

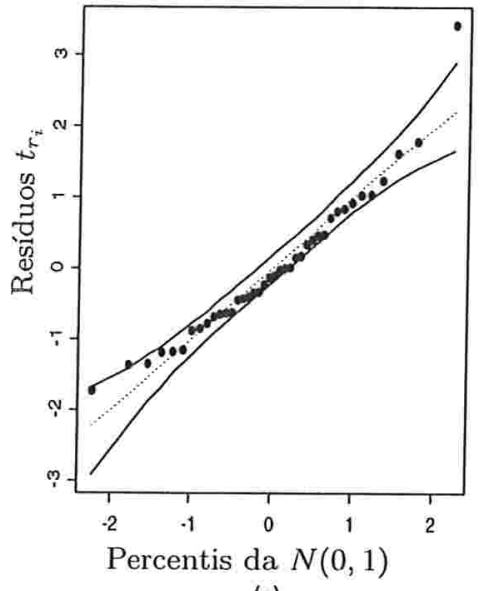

(a)

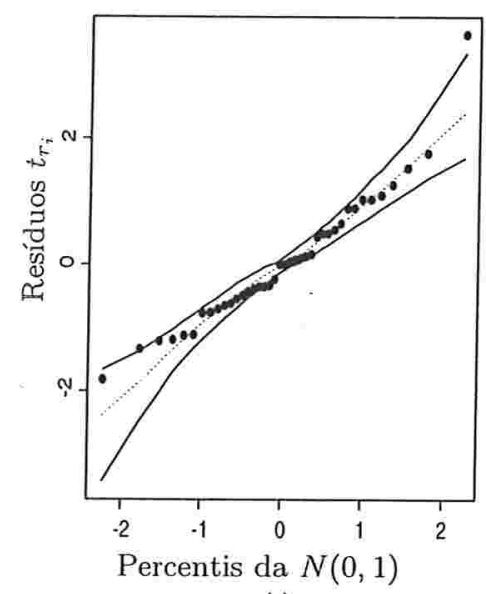

(c)

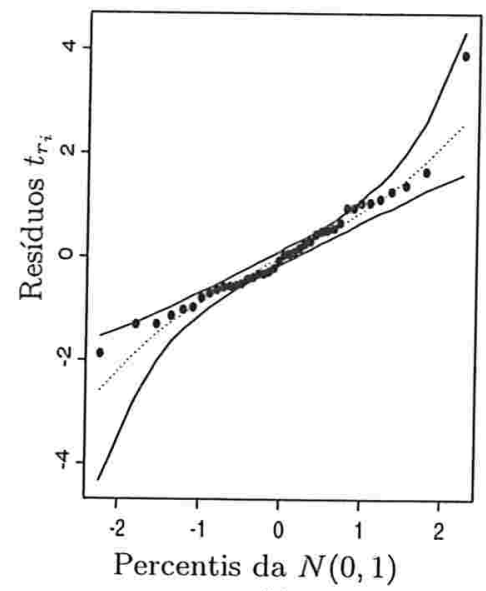

(b)

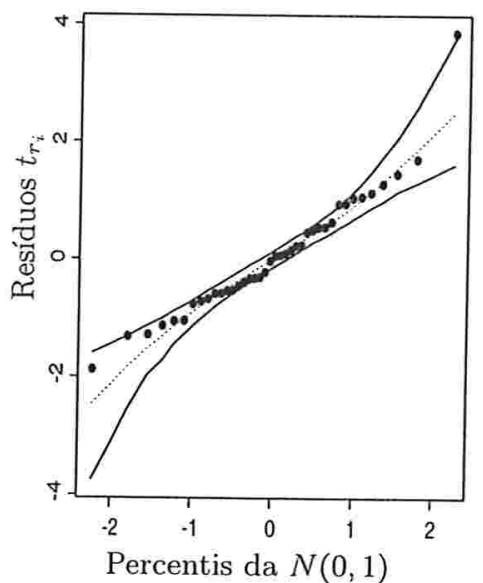

(d) 
Figura 5.3 Gráfico de índices de $\mathrm{C}_{i}$ para as estimativas dos parâmetros do modelo (5.5) sob erro (a) normal, (b) t-Student com 6 g.l., (c) EP(0,3) e (d) logístico-II.

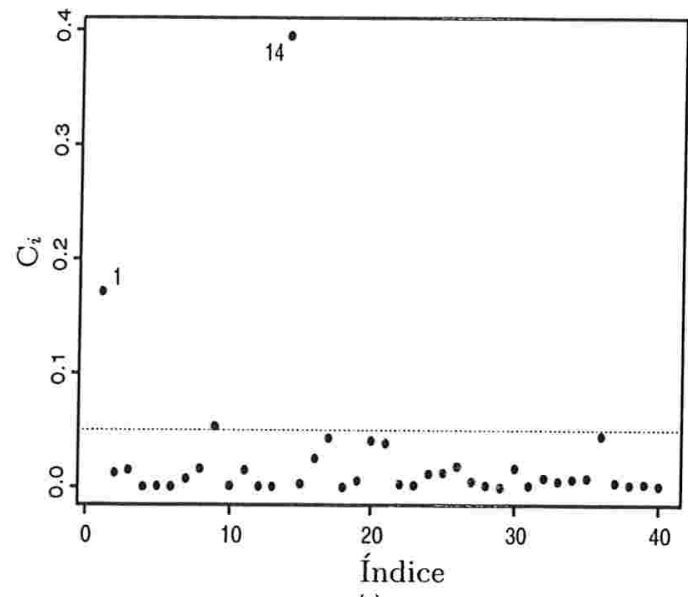

(a)

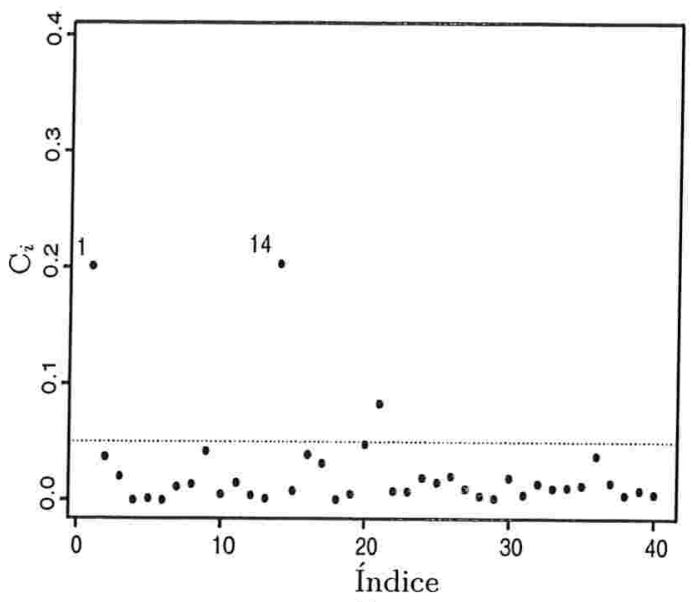

(c)

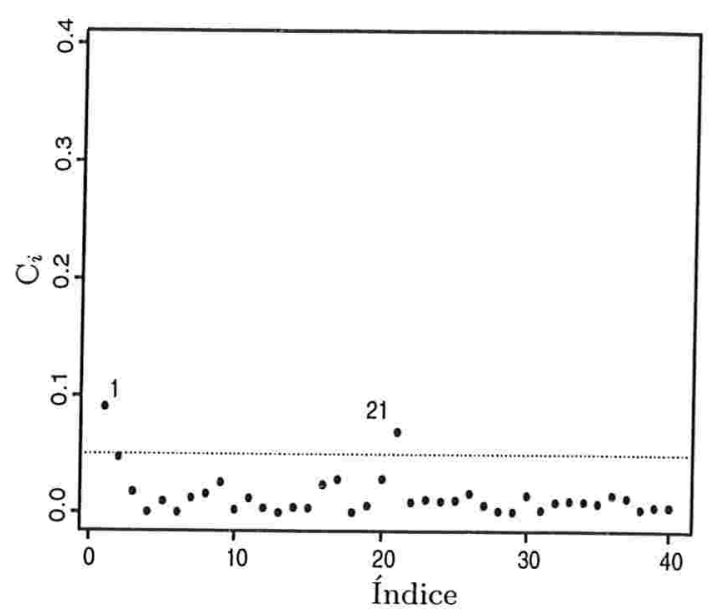

(b)

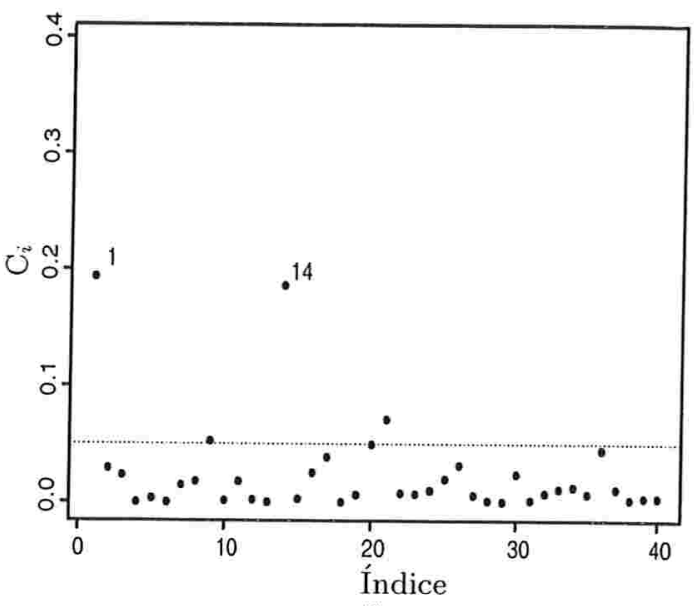

(d) 
Figura 5.4 Gráfico de indices de $\mathrm{C}_{i}(\boldsymbol{\beta})$ para as estimativas dos parâmetros do modelo (5.5) sob erro (a) normal, (b) t-Student com 6 g.l., (c) $E P(0,3)$ e (d) Logístico-II.

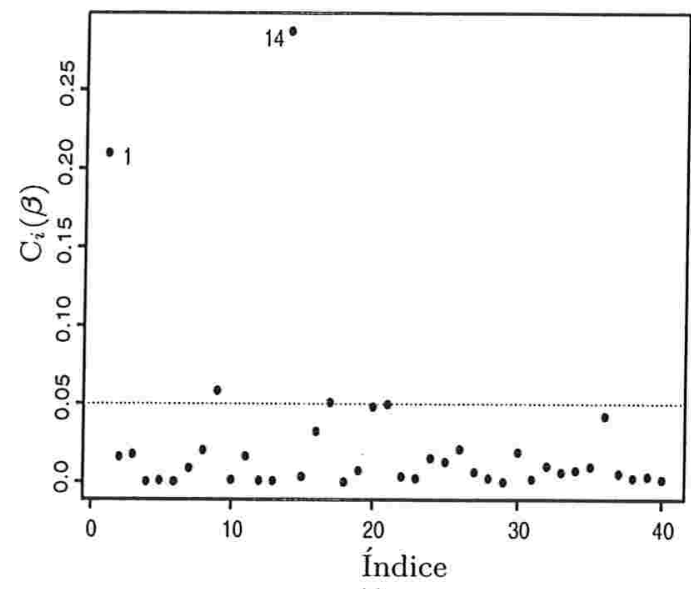

(a)

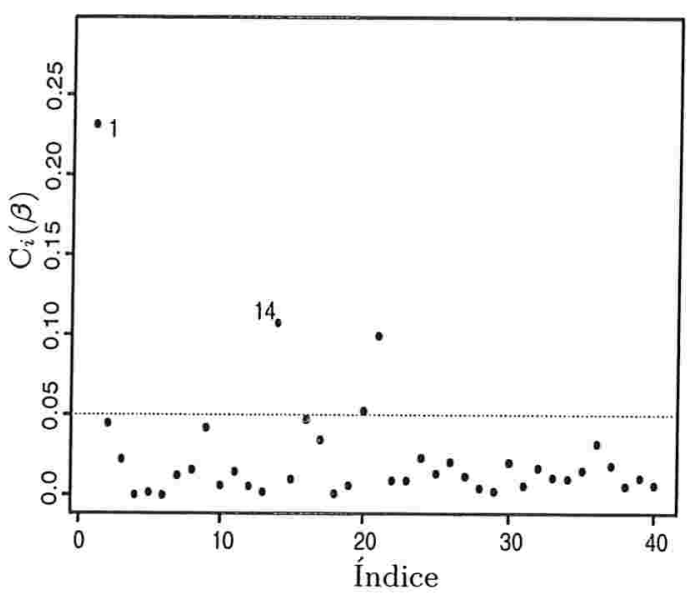

(c)

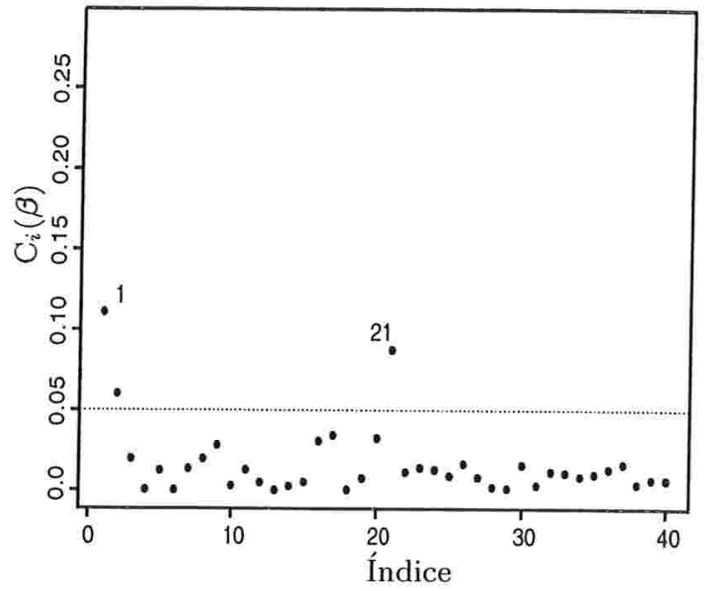

(b)

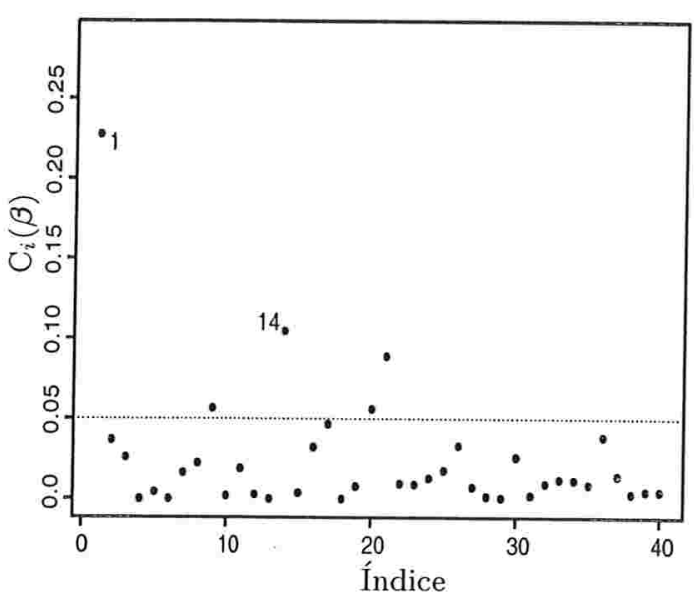

(d) 
Figura 5.5 Gráfico de índices de $\mathrm{C}_{i}(\phi)$ para as estimativas dos parâmetros do modelo (5.5) sob erro (a) normal, (b) t-Student com 6 g.l., (c) $\operatorname{EP}(0,3)$ e (d) Logístico-II.

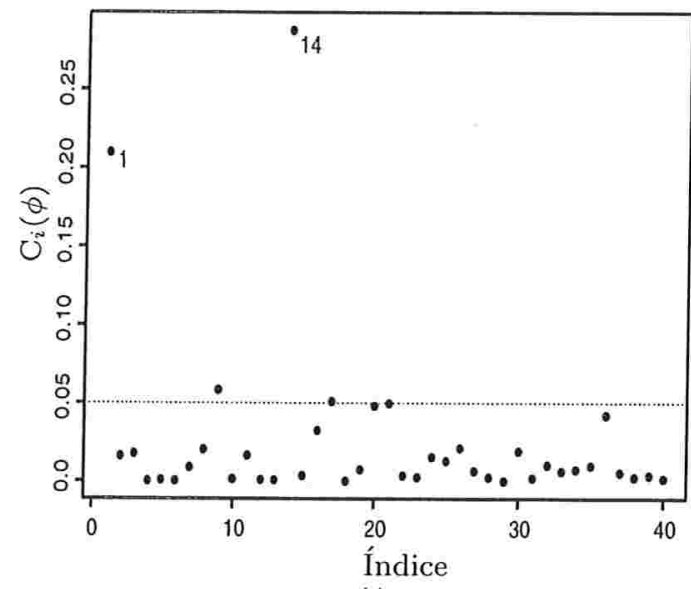

(a)

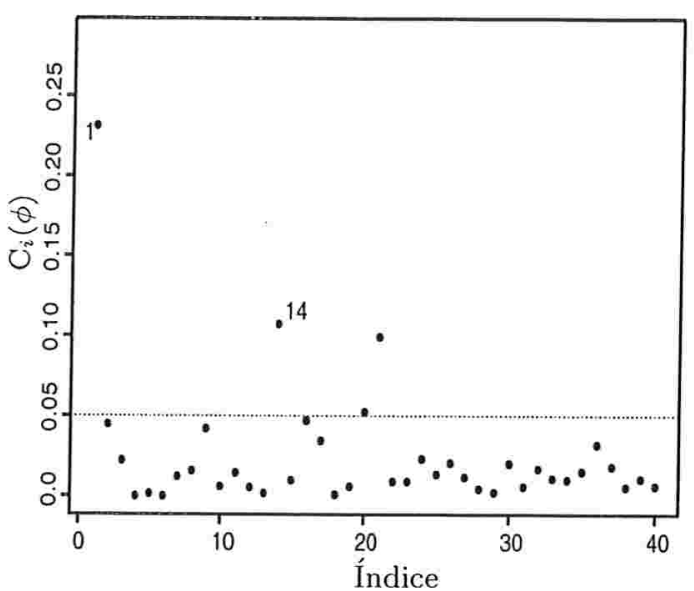

(c)

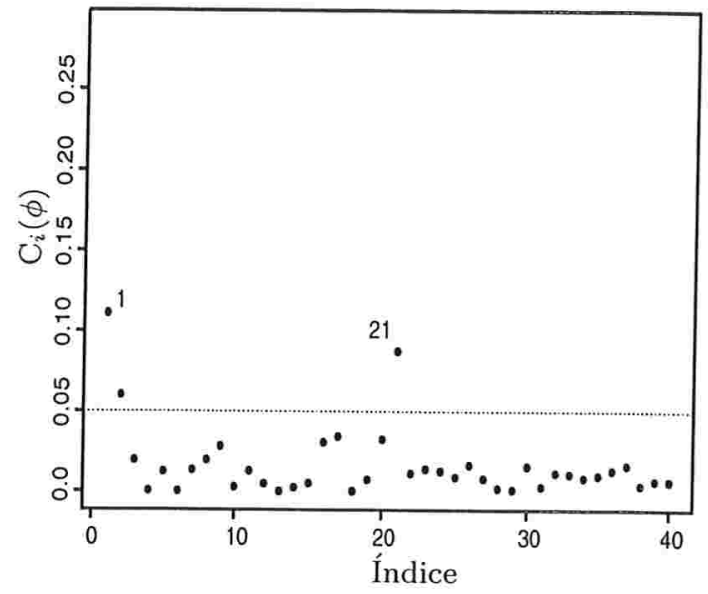

(b)

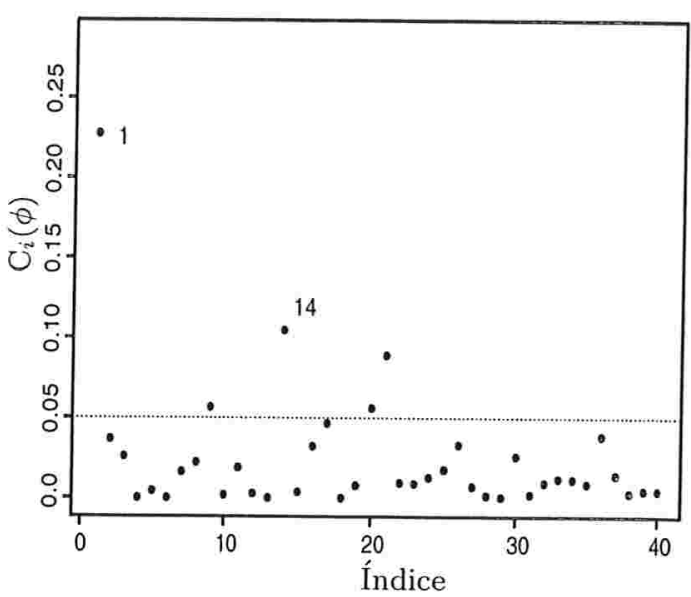

(d) 
Tabela 5.6 Mudanças (em \%) nas estimativas dos parâmetros dos modelos irrestritos ajustados aos dados de TV a cabo excluindo as áreas 1 e 14 (erros padrões).

\begin{tabular}{c|cccc}
\hline Parâmetro & Normal & $t_{6}$ & $\mathrm{EP}(0,3)$ & Logístico-II \\
\hline$\beta_{0}$ & 80,34 & 22,37 & 52,60 & 30,77 \\
& $(-23,92)$ & $(-16,56)$ & $(-20,98)$ & $(-18,46)$ \\
$\beta_{1}$ & 10,25 & 13,03 & 13,77 & 12,58 \\
& $(-14,60)$ & $(-6,34)$ & $(-11,30)$ & $(-8,48)$ \\
$\beta_{2}$ & $-60,10$ & $-44,03$ & $-53,54$ & $-47,37$ \\
& $(-24,31)$ & $(-16,99)$ & $(-21,39)$ & $(-18,88)$ \\
$\beta_{3}$ & $-140,56$ & $-287,74$ & $-173,80$ & $-225,81$ \\
& $(-22,26)$ & $(-14,75)$ & $(-19,26)$ & $(-16,69)$ \\
$\beta_{4}$ & 30,14 & 4,89 & 19,37 & 9,36 \\
& $(-21,87)$ & $(-14,31)$ & $(-18,85)$ & $(-16,27)$ \\
$\beta_{5}$ & $-33,37$ & $-35,33$ & $-34,28$ & $-34,24$ \\
& $(-21,93)$ & $(-14,39)$ & $(-18,92)$ & $(-16,34)$ \\
$\beta_{6}$ & $-38,91$ & $-40,19$ & $-39,46$ & $-39,41$ \\
& $(-19,70)$ & $(-11,93)$ & $(-16,60)$ & $(-13,94)$ \\
$\phi$ & $-44,80$ & $-33,62$ & $-40,47$ & $-36,61$ \\
& $(-43,37)$ & $(-31,90)$ & $(-38,92)$ & $(-34,96)$ \\
\hline
\end{tabular}

A principal conclusão deste exemplo é que a transformação $\sqrt{y}$ parece estabilizar a variância da resposta, e mais, as distribuições $t$-Student, exponencial potência e logística-II são menos influenciadas pelas observações discrepantes 1 e 14 . Os testes unilaterais baseados nesses três modelos ajustados indicam para a rejeição da hipóteses nula ao nível de significância de 5\%, enquanto sob o modelo normal a rejeição da hipótese nula torna-se mais evidente somente depois da retirada da observação discrepante 14. Contudo, o modelo $t$-Student parece ser o mais robusto contra as observações influentes 1 e 14 do que os outros três modelos. Isso pode ser verificado pelas variações nas estimativas dos parâmetros descritas na Tabela 5.6. 
As grandes variações nas estimativas de $\beta_{3}$ podem ser devidas a esse coeficientes não ser significativo. Se continuarmos o processo de seleção com o modelo $t$-Student somente os coeficientes $\beta_{2}, \beta_{3}$ e $\beta_{4}$ são removidos do modelo. Então, o modelo final fica dado por $\sqrt{y}_{i}=\mu_{i}+\epsilon_{i}$, em que $\epsilon_{i} \sim t_{6}(0, \phi)$ com $\mu_{i}=\beta_{0}+\beta_{1} x_{i 1}+\beta_{5} x_{i 5}+\beta_{6} x_{i 6}$ e $\hat{\beta}_{0}=2,998(0,517), \hat{\beta}_{1}=0,034(0,002), \hat{\beta}_{5}=0,105(0,050), \hat{\beta}_{6}=-0,313(0,094)$ e $\hat{\phi}=0,731(0,200)$.

\subsection{Estudo de sensitividade}

É bem conhecido que distribuições de erros com caudas mais pesadas do que a normal tendem a ser robustas contra observações aberrantes, contudo pouco tem sido investigado sobre a robustez de tais distribuições contra observações influentes ou de alta alavanca. A fim de tentar esclarecer esse ponto para alguns erros simétricos, apresentaremos, a seguir, um estudo de sensitividade em que algumas observações são perturbadas no sentido de se tornarem influentes na estimativa da inclinação do modelo de regressão linear simples $y_{i}=\alpha+\beta x_{i}+\epsilon_{i}$, em que $\epsilon_{i} \sim S(0, \phi)$. O comportamento da estatística da razão verossimilhanças será estudado.

Para algumas configurações particulares para $\alpha, \beta$ e $\phi$ e $n=50, x_{i}$ foi gerado de uma distribuição uniforme no intervalo $(0,1)$. Consideramos para ilustrar $\epsilon_{i}$ seguindo distribuições normal, $t$-Student com 12 e 3 graus de liberdade, exponencial potência com $k=0,3$ e $k=0,6$ e logística-II. Para a distribuição exponencial potência com $k>0$, o coeficiente de afastamento de curtose $\gamma_{2}$ é positivo (leptocúrtica) e cresce com $k$ e para $k<0$ tem-se $\gamma_{2}<0$ (platicúrtica). A distribuição logística-II tem coeficiente de afastamento de curtose $\gamma_{2}=1,2$ maior do que o coeficiente da distribuição normal $\left(\gamma_{2}=0\right)$. Após gerarmos a variável explanatória $x_{i}$, aplicamos um esquema de perturbação no maior valor da variável explanatória, $x_{\max }$, a fim de fazê-lo ponto de alta alavanca, de modo que $x_{\max } \leftarrow x_{\max }+a \sigma_{x}$ para $a \in[0,3]$. Então, aplicamos testes unilaterais para avaliar $\mathrm{H}_{0}: \beta=\beta_{0}$ contra $\mathrm{H}_{2}: \beta>\beta_{0}$, em que $\beta_{0}$ foi escolhido apropriadamente para cada distribuição dos erros tal que o $p$-valor ficasse próximo de $5 \%$ para $a=0$. Para confirmar a in- 
fluência de $x_{\max }$ sob $a=3$, calculamos os elementos da diagonal principal da matriz generalizada de pontos de alavanca $\mathrm{GL}(\hat{\boldsymbol{\theta}})$, definida em Paula, Cysneiros e Galea (2003). O gráfico de índices dos elementos da diagonal principal GL ${ }_{i i}$ é apresentado na Figura 5.6. O comportamento da variação do $p$-valor para $\xi_{R V}$ é descrito na Figura 5.7. Como podemos notar os modelos $t$-Student com 3 graus de liberdade, exponencial potência com $k=0,3$ e logística-II parecem ser mais robustos contra o esquema de perturbação aplicado, sugerindo que os modelos simétricos com caudas mais pesadas do que a normal podem ser também robustos contra pontos de alta alavanca e influentes.

Figura 5.6 Gráfico de indices de $\mathrm{GL}_{i i}$ para o modelo simétrico perturbado $(a=3)$ sob erros (a) normal (b) $t$-Student com 3 g.l., (c) t-Student com 12 g.l., (d) $E P(0,3)$, (e) $E P(0,6)$ e (f) logístico-II.

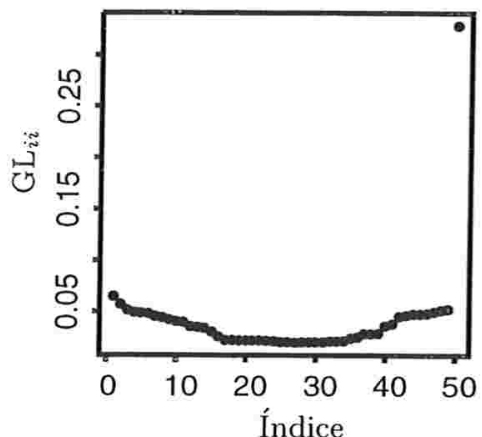

(a)

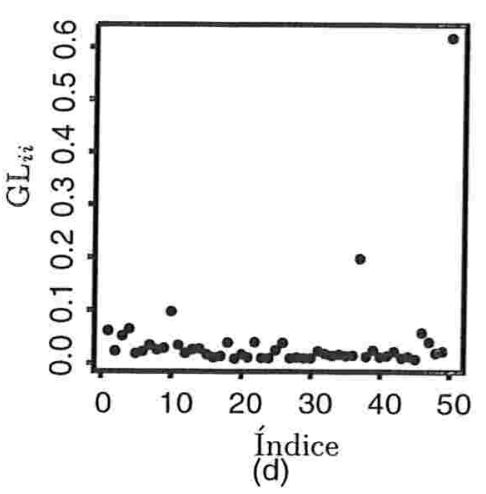

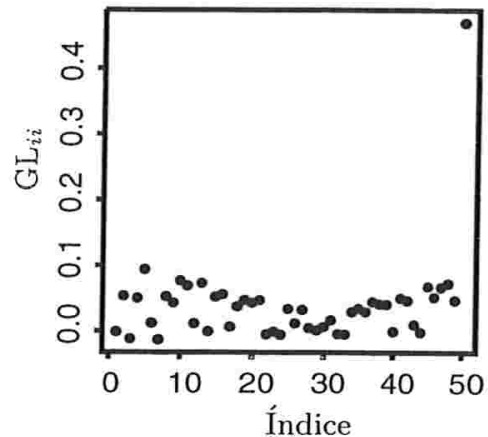

(b)

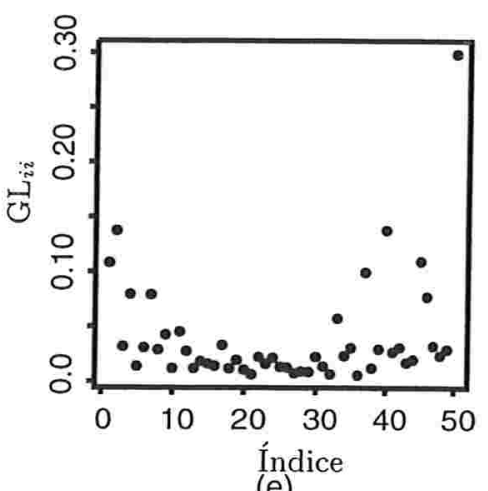

$(\mathrm{e})$

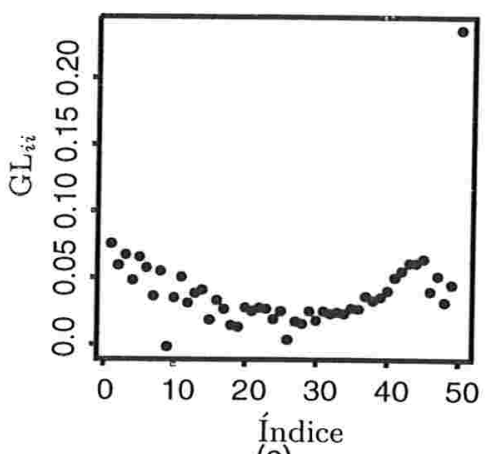

(c)

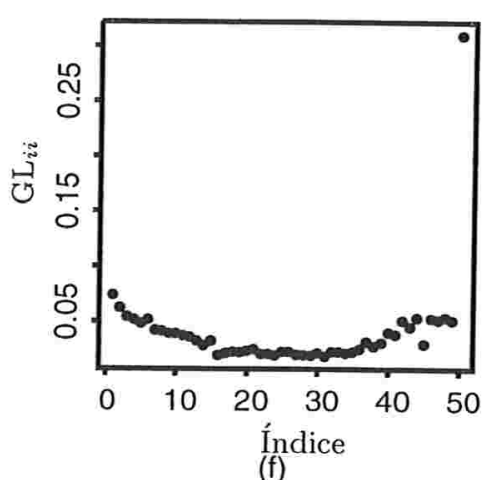


Figura 5.7 Estudo de sensitividade para o p-valor da estatística $\xi_{R V}$ sob perturbações na variável explanatória.
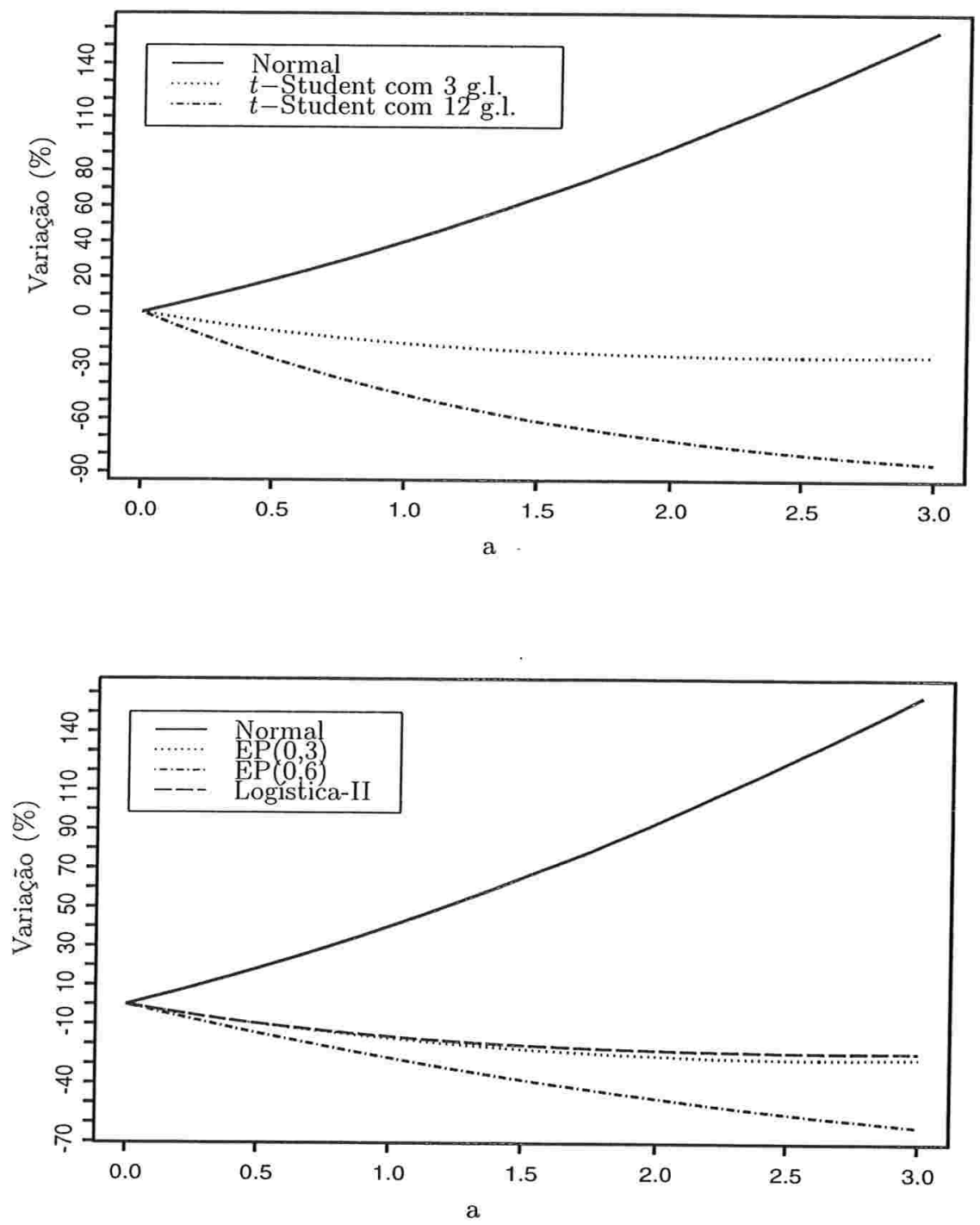


\section{CAPÍTULO 6}

\section{Inferência em modelos $t$-multivariados restritos}

\subsection{Modelo linear $t$-multivariado}

Suponha que temos $n$ indivíduos sendo cada um observado em $m_{i}$ ocasiões. A resposta observada para o $i$-ésimo indivíduo será formada pelo vetor $m_{i} \times 1$ $\mathrm{y}_{i}=\left(y_{i 1}, \ldots, y_{i m_{i}}\right)^{T}$. Além disso, assumiremos o modelo linear

$$
\mathrm{y}_{i}=\mathrm{X}_{i} \beta+\epsilon_{i}, \quad i=1, \ldots, n
$$

em que $\mathbf{X}_{i}$ é uma matriz de planejamento conhecida de dimensão $m_{i} \times p, \boldsymbol{\beta}=$ $\left(\beta_{1}, \ldots, \beta_{p}\right)^{T}$ e os $\epsilon_{i}$ 's são vetores $m_{i}$-dimensionais de erros independentes. Cada $\epsilon_{i}$ é assumido seguir uma distribuição $t$-multivariada $t_{m_{i}}\left(0, \Phi_{i}, \nu_{i}\right)$, cuja função de densidade toma a forma

$$
f_{\epsilon_{i}}(\epsilon)=\frac{\Gamma\left\{\left(\nu_{i}+m_{i}\right) / 2\right\}}{\Gamma\left(\nu_{i} / 2\right)\left(\pi \nu_{i}\right)^{m_{i} / 2}\left|\Phi_{i}\right|^{1 / 2}}\left[1+\frac{1}{\nu_{i}}\left\{\epsilon_{i}^{T} \Phi_{i}^{-1} \epsilon_{i}\right\}\right]^{-\left(\nu_{i}+m_{i}\right) / 2},
$$

em que $\Phi_{i}$ é uma matriz escala e os $\nu_{i}$ 's representam os graus de liberdade. Portanto, segue de Lange, Little e Taylor (1989) que $\mathrm{E}\left(\mathrm{y}_{i}\right)=\mathbf{X}_{i} \boldsymbol{\beta}\left(\nu_{i}>1\right)$ e $\operatorname{Var}\left(\mathrm{y}_{i}\right)=\frac{\nu_{i}}{\nu_{i}-2} \Phi_{i}\left(\nu_{i}>2\right)$. Podemos parametrizar a matriz $\Phi_{i}$ assumindo que $\Phi_{i}=\sigma^{2} \mathbf{R}_{i}$, em que $\sigma^{2}>0$ é um parâmetro de escala e $\mathbf{R}_{i}=\mathbf{R}_{i}(\rho)$ é uma matriz de correlação para cada indivíduo reparametrizada por um conjunto de parâmetros $\rho=\left(\rho_{1}, \ldots, \rho_{q}\right)^{T}$ que não dependem de $\boldsymbol{\beta}$. Assumiremos, também, que os $\nu_{i}$ 's são fixados. Os modelos definidos por (6.1) e (6.2) serão chamados de modelos lineares $t$-multivariados.

Modelos lineares e não-lineares com distribuição $t$-multivariada para os erros têm sido investigados em vários contextos. Por exemplo, Lange, Little e Taylor (1989) apresentam inferência básica para modelos $t$-multivariados com graus de liberdade desconhecidos; mais recentemente, Kowalski, Mendonza-Blanco, Tu 
e Gleser (1999) comparam inferência clássica e Bayesiana em modelos lineares t-multivariados, enquanto Fernandez e Steel (1999) revelam alguns problemas de ambos os métodos em modelos lineares $t$-multivariados com graus de liberdade desconhecidos. Pinheiro, Liu e Wu (2001) propõem um modelo linear robusto hierárquico de efeitos mistos, em que o efeito aleatório e o erro seguem distribuição $t$-multivariada.

O logaritmo da função de verossimilhança para o modelo (6.1) pode ser escrito como

$$
\begin{aligned}
\mathrm{L}\left(\boldsymbol{\beta}, \sigma^{2}, \rho\right)= & \sum_{i=1}^{n} \log \Upsilon_{i}-\frac{n}{2} \bar{m} \log \sigma^{2}-\frac{1}{2} \sum_{i=1}^{n} \log \left|\mathbf{R}_{i}\right| \\
& +\sum_{i=1}^{n} \log \left\{1+\frac{1}{\sigma^{2} \nu_{i}}\left(\mathbf{y}_{i}-\mathbf{X}_{i} \boldsymbol{\beta}\right)^{T} \mathbf{R}_{i}^{-1}\left(\mathbf{y}_{i}-\mathbf{X}_{i} \boldsymbol{\beta}\right)\right\}^{-\frac{\nu_{i}+m_{i}}{2}}
\end{aligned}
$$

em que $\Upsilon_{i}=\Gamma\left(\frac{\nu_{i}+m_{i}}{2}\right) /\left[\left(\pi \nu_{i}\right)^{m_{i} / 2} \Gamma\left(\frac{\nu_{i}}{2}\right)\right], \nu=\left(\nu_{1}, \ldots, \nu_{n}\right)^{T}, \mathrm{y}=\left(y_{1}, \ldots, y_{n}\right)^{T} \mathrm{e}$ $\Gamma(\cdot)$ é a função gama. Denotando $\omega_{i}=\left(\nu_{i}+m_{i}\right) /\left\{\nu_{i}+\mathrm{D}_{R_{i}}\left(\mathrm{y}_{i}, \mathrm{X}_{i}, \boldsymbol{\beta}\right)\right\}$, em que $\mathrm{D}_{R_{i}}\left(\mathrm{y}_{i}, \mathrm{X}_{i}, \boldsymbol{\beta}\right)=\left(\mathrm{y}_{i}-\mathrm{X}_{i} \boldsymbol{\beta}\right)^{T} \mathbf{R}_{i}^{-1}\left(\mathrm{y}_{i}-\mathrm{X}_{i} \boldsymbol{\beta}\right) / \sigma^{2}$ é a distância de Mahalanobis, a função escore para $\beta, \sigma^{2}$ e $\rho$ fica, respectivamente, dada por

$$
\begin{aligned}
\mathrm{U}_{\beta}\left(\boldsymbol{\beta}, \sigma^{2}, \rho\right) & =\frac{1}{\sigma^{2}} \sum_{i=1}^{n} \omega_{i} \mathrm{X}_{i}^{T} \mathbf{R}_{i}^{-1}\left(\mathbf{y}_{i}-\mathrm{X}_{i} \boldsymbol{\beta}\right) \\
\mathrm{U}_{\sigma^{2}}\left(\boldsymbol{\beta}, \sigma^{2}, \rho\right) & =-\frac{n \bar{m}}{2 \sigma^{2}}+\frac{1}{\sigma^{2}} \sum_{i=1}^{n}\left(\frac{\nu_{i}+m_{i}}{2}\right)\left\{\frac{\mathrm{D}_{R_{i}}\left(\mathrm{y}_{i}, \mathrm{X}_{i}, \boldsymbol{\beta}\right)}{\nu_{i}+\mathrm{D}_{R_{i}}\left(\mathrm{y}_{i}, \mathrm{X}_{i}, \boldsymbol{\beta}\right)}\right\} \\
& =-\frac{n \bar{m}}{2 \sigma^{2}}+\frac{1}{2 \sigma^{2}} \sum_{i=1}^{n} \omega_{i} \mathrm{D}_{R_{i}}\left(\mathrm{y}_{i}, \mathrm{X}_{i}, \boldsymbol{\beta}\right) \\
\mathrm{U}_{\rho}\left(\boldsymbol{\beta}, \sigma^{2}, \rho\right) & =\left(\mathrm{U}_{\rho_{1}}\left(\boldsymbol{\beta}, \sigma^{2}, \boldsymbol{\rho}\right), \ldots, \mathrm{U}_{\rho_{q}}\left(\boldsymbol{\beta}, \sigma^{2}, \boldsymbol{\rho}\right)\right)^{T}
\end{aligned}
$$

em que

$\mathrm{U}_{\rho_{j}}\left(\boldsymbol{\beta}, \sigma^{2}, \rho\right)=-\frac{1}{2} \sum_{i=1}^{n} \operatorname{tr}\left(\mathbf{R}_{i}^{-1} \frac{\partial \mathbf{R}_{i}}{\partial \rho_{j}}\right)+\frac{1}{2 \sigma^{2}} \sum_{i=1}^{n} \omega_{i}\left(\mathbf{y}_{i}-\mathbf{X}_{i} \boldsymbol{\beta}\right)^{T} \mathbf{R}_{i}^{-1} \frac{\partial \mathbf{R}_{i}}{\partial \rho_{j}} \mathbf{R}_{i}^{-1}\left(\mathbf{y}_{i}-\mathbf{X}_{i} \boldsymbol{\beta}\right)$ para $j=1, \ldots, q$. 
A matriz de informação de Fisher para $\theta=\left(\beta^{T}, \sigma^{2}, \rho^{T}\right)^{T}$ é dada por

$$
\mathbf{K}_{\theta \theta}=\left[\begin{array}{rrr}
\mathbf{K}_{\beta \beta} & 0 & 0 \\
0 & \mathrm{~K}_{\sigma^{2} \sigma^{2}} & \mathbf{K}_{\sigma^{2} \rho} \\
0 & \mathbf{K}_{\rho \sigma^{2}} & \mathbf{K}_{\rho \rho}
\end{array}\right]
$$

em que

$$
\begin{aligned}
\mathbf{K}_{\beta \beta} & =\frac{1}{\sigma^{2}} \sum_{i=1}^{n}\left(\frac{\nu_{i}+m_{i}}{\nu_{i}+m_{i}+2}\right) \mathbf{X}_{i}^{T} \mathbf{R}_{i}^{-1} \mathbf{X}_{i} \\
\mathrm{~K}_{\sigma^{2} \sigma^{2}} & =\frac{1}{2 \sigma^{4}} \sum_{i=1}^{n} \frac{m_{i} \nu_{i}}{\nu_{i}+m_{i}+2}
\end{aligned}
$$

$\mathrm{K}_{\sigma^{2} \rho}$ é um vetor $(1 \times q)$ em que o $\ell$-ésimo elemento é dado por

$$
\mathrm{K}_{\sigma^{2} \rho_{\ell}}=\frac{1}{2 \sigma^{2}} \sum_{i=1}^{n}\left(\frac{\nu_{i}}{\nu_{i}+m_{i}+2}\right) \operatorname{tr}\left(\mathbf{R}_{i}^{-1} \frac{\partial \mathbf{R}_{i}}{\partial \rho_{\ell}}\right) \quad \mathrm{e}
$$

$\mathrm{K}_{\rho \rho}$ é uma matriz $(q \times q)$ em que o $(\ell, s)$-ésimo elemento fica expresso na forma

$$
\begin{aligned}
\mathrm{K}_{\rho_{\ell} \rho_{s}}= & \frac{1}{2} \sum_{i=1}^{n}\left\{\left(\frac{\nu_{i}+m_{i}}{\nu_{i}+m_{i}+2}\right) \operatorname{tr}\left(\mathbf{R}_{i}^{-1} \frac{\partial \mathbf{R}_{i}}{\partial \rho_{\ell}} \mathbf{R}_{i}^{-1} \frac{\partial \mathbf{R}_{i}}{\partial \rho_{s}}\right)\right. \\
& \left.-\left(\frac{1}{\nu_{i}+m_{i}+2}\right) \operatorname{tr}\left(\mathbf{R}_{i}^{-1} \frac{\partial \mathbf{R}_{i}}{\partial \rho_{\ell}}\right) \operatorname{tr}\left(\mathbf{R}_{i}^{-1} \frac{\partial \mathbf{R}_{i}}{\partial \rho_{s}}\right)\right\} .
\end{aligned}
$$

Pode-se mostrar que $\boldsymbol{\beta}$ e $\rho$ são parâmetros ortogonais como também $\beta$ e $\sigma^{2}$. O processo iterativo conjunto para resolver $\mathrm{U}_{\beta}\left(\boldsymbol{\beta}, \sigma^{2}, \rho\right)=0, \mathrm{U}_{\sigma^{2}}\left(\boldsymbol{\beta}, \sigma^{2}, \rho\right)=0$ e $\mathrm{U}_{\rho}\left(\boldsymbol{\beta}, \sigma^{2}, \rho\right)=0$ é dado por

$$
\boldsymbol{\beta}^{(r+1)}=\left(\sum_{i=1}^{n} \omega_{i}^{(r)} \mathbf{X}_{i}^{T} \mathbf{R}_{i}^{-(r)} \mathbf{X}_{i}\right)^{-1} \sum_{i=1}^{n} \omega_{i}^{(r)} \mathbf{X}_{i}^{T} \mathbf{R}_{i}^{-(r)} \mathbf{y}_{i}
$$

e

$$
\boldsymbol{\theta}^{(r+1)}=\operatorname{argmax}_{\boldsymbol{\theta}}\left\{\mathrm{L}\left(\beta^{(r+1)}, \theta\right)\right\}, \operatorname{com} \quad \boldsymbol{\theta}=\left(\sigma^{2}, \rho^{T}\right)^{T}
$$

para $r=0,1, \ldots$.

Deve-se iniciar o processo iterativo (6.3)-(6.4) com valores iniciais $\boldsymbol{\beta}^{(0)}, \sigma^{2(0)} \mathrm{e}$ $\rho^{(0)}$. Para resolver (6.4) podemos aplicar algum algoritmo de maximização, tal como quasi-Newton, scoring-Fisher ou EM. Temos trabalhado com o algoritmo 
quasi-Newton, BFGS implementado no software Ox (veja, por exemplo, Doornik, 1999). Note que, de (6.3) o peso $\omega_{i}^{(r)}$, é inversamente proporcional à distância de Mahalanobis, de forma que observações aberrantes tendem a ter pesos menores no processo de estimação. Portanto, é esperado que o processo iterativo (6.3) forneça estimativas mais robustas contra pontos aberrantes do que o caso normal. Segue sob condições de regularidade (veja, por exemplo, Maronna, 1976; Lange, Little e Taylor, 1989 e Kowalski, Mendonza-Blanco, Tu e Gleser, 1999) que $\hat{\boldsymbol{\beta}}$ é um estimador consistente $\beta$, e

$$
\sqrt{n}(\hat{\boldsymbol{\beta}}-\boldsymbol{\beta}) \stackrel{\mathrm{d}}{\rightarrow} \mathrm{N}_{p}\left(0, \mathbf{J}_{\beta \beta}^{-1}\right), \text { em que } \mathbf{J}_{\beta \beta}=\lim _{n \rightarrow \infty} \frac{1}{n} \mathbf{K}_{\beta \beta} .
$$

Então, $\hat{\mathbf{K}}_{\beta \beta}^{-1}=\left\{\frac{1}{\hat{\sigma}^{2}} \sum_{i=1}^{n}\left(\frac{\nu_{i}+m_{i}}{\nu_{i}+m_{i}+2}\right)\left(\mathbf{X}_{i}^{T} \hat{\mathbf{R}}_{i}^{-1} \mathbf{X}_{i}\right)\right\}^{-1}$ é um estimador consistente da matriz de variância-covariancia assintótica de $\hat{\boldsymbol{\beta}}$. Assumiremos que $\nu_{i}=\nu$ e que $\nu$ é substituído por um estimador consistente na expressão acima.

\section{$6.2 g$ grupos}

Suponha que temos agora $g$ grupos, tal que cada um tenha $n_{\ell}$ indivíduos que são observados nas mesmas $m$ ocasiões. A resposta observada para o $i$-ésimo indivíduo do $\ell$-ésimo grupo formado é dado por $\mathrm{y}_{i \ell}=\left(y_{i \ell 1}, \ldots, y_{i \ell m}\right)^{T}, i=1, \ldots, n_{\ell}$ e $\ell=$ $1, \ldots, g$. Além disso, assumiremos o modelo

$$
\mathrm{y}_{i \ell}=\mu_{\ell} \mathbf{1}_{m}+\epsilon_{i \ell}
$$

em que $\mathbf{1}_{m}$ é um vetor $m \times 1$ de uns e $\epsilon_{i \ell} \sim t_{m}\left(0, \Phi_{i \ell}, \nu_{\ell}\right)$ com $\Phi_{i \ell}=\sigma^{2} \mathbf{R}(\rho)$ e $\sigma^{2}>0$, em que $\mathbf{R}(\rho)$ denota a matriz de correlação para cada indivíduo, $j=1, \ldots, m$. Portanto, temos que $\mathrm{E}\left(y_{i \ell j}\right)=\mu_{\ell}$ e $\operatorname{Var}\left(y_{i \ell j}\right)=\frac{\nu_{\ell}}{\nu_{\ell}-2} \sigma^{2}$. A estimativa de máxima verossimilhança de $\mu_{\ell}$ toma aqui a forma

$$
\hat{\mu}_{\ell}=\left\{\sum_{i=1}^{n_{\ell}} \hat{\omega}_{i \ell} \mathbf{1}_{m}^{T} \mathbf{R}^{-1}(\hat{\rho}) \mathbf{1}_{m}\right\}^{-1}\left\{\sum_{i=1}^{n_{\ell}} \hat{\omega}_{i \ell} 1_{m}^{T} \mathbf{R}^{-1}(\hat{\rho}) \mathbf{y}_{i \ell}\right\},
$$

em que $\omega_{i \ell}=\left(\nu_{\ell}+m\right) /\left\{\nu_{\ell}+\mathrm{D}_{R}\left(\mathbf{y}_{i \ell}, \mu_{\ell}\right)\right\} \operatorname{com} \mathrm{D}_{R}\left(\mathrm{y}_{i \ell}, \mu_{\ell}\right)=\frac{1}{\sigma^{2}}\left(\mathrm{y}_{i \ell}-\mu_{\ell} \mathbf{1}_{m}\right)^{T} \mathbf{R}^{-1}\left(\mathrm{y}_{i \ell}-\right.$ $\mu_{\ell} 1_{m}$ ). Assumindo que $\frac{n_{\ell}}{n} \rightarrow \gamma_{\ell}, \forall \ell$, quando $n \rightarrow \infty$ segue que sob condições de 
regularidade que

$$
\sqrt{n}(\hat{\mu}-\mu) \stackrel{\mathrm{d}}{\rightarrow} \mathrm{N}_{g}\left(0, \mathrm{~J}_{\mu}^{-1}\right)
$$

em que $\boldsymbol{\mu}=\left(\mu_{1}, \ldots, \mu_{g}\right)^{T}, n=n_{1}+\cdots+n_{g}$ e $\mathbf{J}_{\mu}=\operatorname{diag}\left\{v_{1}^{2}, \ldots, v_{g}^{2}\right\}$ com

$$
\begin{aligned}
v_{\ell}^{2} & =\lim _{n \rightarrow \infty} \frac{1}{n} \sum_{i=1}^{n_{\ell}} \frac{1}{\sigma^{2}}\left(\frac{\nu_{\ell}+m}{\nu_{\ell}+m+2}\right)\left(\mathbf{1}_{m}^{T} \mathbf{R}^{-1} \mathbf{1}_{m}\right) \\
& =\frac{\gamma_{\ell}}{\sigma^{2}}\left(\frac{\nu_{\ell}+m}{\nu_{\ell}+m+2}\right)\left(\mathbf{1}_{m}^{T} \mathbf{R}^{-1} \mathbf{1}_{m}\right) .
\end{aligned}
$$

Então, um estimador consistente para a variância assintótica de $\hat{\mu}_{\ell}$ é dado por

$$
\begin{aligned}
\hat{v}_{\ell}^{2} & =\left\{\sum_{i=1}^{n_{\ell}} \frac{1}{\hat{\sigma}^{2}}\left(\frac{\nu_{\ell}+m}{\nu_{\ell}+m+2}\right)\left(\mathbf{1}_{m}^{T} \hat{\mathbf{R}}^{-1} \mathbf{1}_{m}\right)\right\}^{-1} \\
& =\frac{\hat{\sigma}^{2}}{n_{\ell}}\left(\frac{\nu_{\ell}+m+2}{\nu_{\ell}+m}\right)\left(\mathbf{1}_{m}^{T} \hat{\mathbf{R}}^{-1} \mathbf{1}_{m}\right)^{-1}
\end{aligned}
$$

em que $\hat{\sigma}^{2}=\frac{1}{n} \sum_{\ell=1}^{g} \sum_{i=1}^{n_{\ell}} \sum_{j=1}^{m} \hat{\omega}_{i \ell}\left(y_{i \ell j}-\hat{\mu}_{\ell}\right)^{2}$ e $\hat{\mathbf{R}}=\mathbf{R}(\hat{\rho})$ pode ser avaliado em algum estimador consistente de $\rho$.

\subsection{Restrições em igualdades lineares}

Suponha, inicialmente, que estamos interessados na estimação do vetor de parâmetros $\boldsymbol{\beta}$ sob $k$ restrições lineares independentes $\mathbf{C}_{j}^{T} \boldsymbol{\beta}-\mathrm{d}_{j}=0$, em que $\mathbf{C}_{j}, j=$ $1, \ldots, k$, são vetores $p \times 1 \mathrm{e} \mathrm{d}_{j}, j=1, \ldots, k$, são escalares, ambos números conhecidos e fixados. O problema aqui é maximizar o logaritmo de função de verossimilhança $\mathrm{L}\left(\boldsymbol{\beta}, \sigma^{2}, \rho\right)$ sujeito a restrições lineares $\mathbf{C} \boldsymbol{\beta}-\mathbf{d}=\mathbf{0}$, em que $\mathbf{C}=$ $\left(\mathrm{C}_{1}^{T}, \ldots, \mathbf{C}_{k}^{T}\right)^{T}$ e $\mathrm{d}=\left(\mathrm{d}_{1}, \ldots, \mathrm{d}_{k}\right)^{T}$. Considere a função penalizada quadrática

$$
\mathrm{P}\left(\boldsymbol{\beta}, \sigma^{2}, \boldsymbol{\rho}, \psi\right)=\mathrm{L}\left(\boldsymbol{\beta}, \sigma^{2}, \boldsymbol{\rho}\right)-\frac{1}{2} \sum_{j=1}^{k} \psi_{j}\left(\mathrm{~d}_{j}-\mathrm{C}_{j}^{T} \beta\right)^{2} .
$$

O procedimento de maximização como foi visto na Seção 5.2 , consiste em encontrar a solução de $\max _{\left\{\beta, \sigma^{2}, \rho\right\}} \mathrm{P}\left(\boldsymbol{\beta}, \sigma^{2}, \boldsymbol{\rho}, \psi\right)$ para valores positivos e fixados de $\psi_{j}$, $j=1, \ldots, k$. A solução para $\beta$ será denotada por $\beta(\psi)$. A estimativa restrita por igualdades é dado por

$$
\hat{\boldsymbol{\beta}}^{0}=\lim _{\psi_{1}, \ldots, \psi_{k} \rightarrow \infty} \boldsymbol{\beta}(\psi) .
$$


Sendo assim, pode ser mostrado que $\boldsymbol{\beta}(\psi)$ é a solução do seguinte processo iterativo:

$$
\begin{aligned}
\beta^{0(r+1)}= & \left(\sum_{i=1}^{n} \omega_{i}^{(r)} \mathbf{X}_{i}^{T} \mathbf{R}_{i}^{-(r)} \mathbf{X}_{i}\right)^{-1} \sum_{i=1}^{n} \omega_{i}^{(r)} \mathbf{X}_{i}^{T} \mathbf{R}_{i}^{-(r)} \mathbf{y}_{i}+\left(\sum_{i=1}^{n} \omega_{i}^{(r)} \mathbf{X}_{i}^{T} \mathbf{R}_{i}^{-(r)} \mathbf{X}_{i}\right)^{-1} \\
& \times \mathbf{C}^{T}\left\{\mathrm{C}\left(\sum_{i=1}^{n} \omega_{i}^{(r)} \mathbf{X}_{i}^{T} \mathbf{R}_{i}^{-(r)} \mathbf{X}_{i}\right)^{-1} \mathbf{C}^{T}\right\} \\
& \times\left\{\mathrm{d}-\mathbf{C}\left(\sum_{i=1}^{n} \omega_{i}^{(r)} \mathbf{X}_{i}^{T} \mathbf{R}_{i}^{-(r)} \mathbf{X}_{i}\right)^{-1} \sum_{i=1}^{n} \omega_{i}^{(r)} \mathbf{X}_{i}^{T} \mathbf{R}_{i}^{-(r)} \mathbf{y}_{i}\right\}
\end{aligned}
$$

para $r=0,1, \ldots$, em que $\sigma^{2(r)}$ e $\mathbf{R}_{i}^{(r)}$ são obtidos de (6.4). O processo iterativo (6.7) pode ser, alternativamente, expresso como

$$
\begin{aligned}
\beta^{0(r+1)}= & \mathbf{b}^{(r+1)}+\left(\sum_{i=1}^{n} \omega_{i}^{(r)} \mathrm{X}_{i}^{T} \mathbf{R}_{i}^{-(r)} \mathrm{X}_{i}\right)^{-1} \\
& \times \mathrm{C}^{T}\left\{\mathrm{C}\left(\sum_{i=1}^{n} \omega_{i}^{(r)} \mathrm{X}_{i}^{T} \mathbf{R}_{i}^{-(r)} \mathrm{X}_{i}\right)^{-1} \mathrm{C}^{T}\right\}^{-1}\left\{\mathrm{~d}-\mathrm{Cb}^{(r+1)}\right\}
\end{aligned}
$$

para $r=0,1, \ldots$, em que $\mathbf{b}^{(r+1)}$ denota $\boldsymbol{\beta}^{(r+1)}$ avaliado na estimativa restrita. Desenvolvemos um programa em Ox para resolver o processo iterativo (6.8). Programas em outros softwares tais como SAS, S-Plus e R podem ser facilmente desenvolvidos.

Pode ser mostrado sob condições de regularidade (veja, por exemplo, Gouriéroux e Monfort, 1995, Seção 10.3) que $\hat{\boldsymbol{\beta}}^{0}$ é um estimador consistente de $\boldsymbol{\beta}$, e

$$
\sqrt{n}\left(\hat{\boldsymbol{\beta}}^{0}-\boldsymbol{\beta}\right) \stackrel{\mathrm{d}}{\rightarrow} \mathrm{N}_{p}\left(\mathbf{0},\left(\mathbf{J}_{\boldsymbol{\beta} \beta}^{\mathbf{0}}\right)^{-1}\right)
$$

em que

$$
\mathbf{J}_{\beta \beta}^{0}=\lim _{\psi_{1}, \ldots, \psi_{k} \rightarrow \infty}\left[\lim _{n \rightarrow \infty} \frac{1}{n} \mathrm{E}\left\{-\frac{\partial \mathrm{P}\left(\boldsymbol{\beta}, \sigma^{2}, \rho, \psi\right)}{\partial \boldsymbol{\beta} \partial \boldsymbol{\beta}^{T}}\right\}\right]
$$

e

$$
\mathrm{E}\left\{-\frac{\partial \mathrm{P}\left(\boldsymbol{\beta}, \sigma^{2}, \boldsymbol{\rho}, \psi\right)}{\partial \boldsymbol{\beta} \partial \boldsymbol{\beta}^{T}}\right\}=\frac{1}{\sigma^{2}} \sum_{i=1}^{n}\left(\frac{\nu_{i}+m_{i}}{\nu_{i}+m_{i}+2}\right) \mathrm{X}_{i}^{T} \mathbf{R}_{i}^{-1} \mathrm{X}_{i}+\mathrm{C}^{T} \Psi \mathrm{C},
$$


$\operatorname{com} \Psi=\operatorname{diag}\left\{\psi_{1}, \ldots, \psi_{k}\right\}$. Então, um estimador consistente da matriz de variânciacovariância assintótica de $\hat{\boldsymbol{\beta}}^{0}$ é dado por

$$
\begin{aligned}
& \lim _{\psi_{1}, \ldots, \psi_{k} \rightarrow \infty}\left\{\frac{1}{\sigma^{2}} \sum_{i=1}^{n}\left(\frac{\nu_{i}+m_{i}}{\nu_{i}+m_{i}+2}\right) \mathbf{X}_{i}^{T} \mathbf{R}_{i}^{-1} \mathbf{X}_{i}+\mathbf{C}^{T} \Psi \mathbf{C}\right\}^{-1} \\
= & \mathrm{K}_{\beta \beta}^{-1}\left\{\mathbf{I}_{p}-\mathrm{C}^{T}\left(\mathbf{C K}_{\beta \beta}^{-1} \mathbf{C}^{T}\right)^{-1} \mathbf{C K}_{\beta \beta}^{-1}\right\},
\end{aligned}
$$

que pode ser avaliado em algum estimador consistente de $\beta$, tais como $\hat{\boldsymbol{\beta}}$ ou $\hat{\boldsymbol{\beta}}^{0}$.

Suponha, agora, que estamos interessados em testar as hipóteses $\mathrm{H}_{0}: \mathbf{C} \boldsymbol{\beta}=\mathrm{d}$ contra $\mathrm{H}_{1}: \mathrm{C} \boldsymbol{\beta} \neq \mathrm{d}$. Os métodos mais usuais para testar essas hipóteses lineares são o teste da razão de verossimilhanças, Wald e escore. As estatísticas ficam aqui, respectivamente, expressas nas formas

$$
\begin{aligned}
\xi_{R V}^{*} & =2\left\{\mathrm{~L}\left(\hat{\boldsymbol{\beta}}, \hat{\sigma}^{2}, \hat{\boldsymbol{\rho}}\right)-\mathrm{L}\left(\hat{\boldsymbol{\beta}}^{0}, \hat{\sigma}_{0}^{2}, \hat{\boldsymbol{\rho}}^{0}\right)\right\} \\
\xi_{W}^{*} & =(\mathbf{C} \hat{\boldsymbol{\beta}}-\mathbf{d})^{T} \hat{\operatorname{Var}}^{-1}(\mathbf{C} \hat{\boldsymbol{\beta}})(\mathbf{C} \hat{\boldsymbol{\beta}}-\mathrm{d}) \\
& =(\mathbf{C} \hat{\boldsymbol{\beta}}-\mathrm{d})^{T}\left\{\mathbf{C} \hat{\mathbf{K}}_{\beta \beta}^{-1} \mathrm{C}^{T}\right\}^{-1}(\mathbf{C} \hat{\boldsymbol{\beta}}-\mathrm{d}) \text { e } \\
\xi_{S R}^{*} & =\left\{\mathrm{U}_{\beta}\left(\hat{\boldsymbol{\beta}}^{0}, \hat{\sigma}_{0}^{2}, \hat{\boldsymbol{\rho}}^{0}\right)-\mathrm{U}_{\beta}\left(\hat{\boldsymbol{\beta}}, \hat{\sigma}^{2}, \hat{\boldsymbol{\rho}}\right)\right\}^{T} \hat{\operatorname{Var}}_{0}(\hat{\boldsymbol{\beta}})\left\{\mathrm{U}_{\beta}\left(\hat{\boldsymbol{\beta}}^{0}, \hat{\sigma}_{0}^{2}, \hat{\boldsymbol{\rho}}^{0}\right)-\mathrm{U}_{\beta}\left(\hat{\boldsymbol{\beta}}, \hat{\sigma}^{2}, \hat{\boldsymbol{\rho}}\right)\right\} \\
& =\mathrm{U}_{\beta}\left(\hat{\boldsymbol{\beta}}^{0}, \hat{\sigma}_{0}^{2}, \hat{\boldsymbol{\rho}}^{0}\right)^{T}\left(\hat{\mathbf{K}}_{\beta \beta}^{0}\right)^{-1} \mathrm{U}_{\beta}\left(\hat{\boldsymbol{\beta}}^{0}, \hat{\sigma}_{0}^{2}, \hat{\boldsymbol{\rho}}^{0}\right),
\end{aligned}
$$

em que $\hat{\mathbf{K}}_{\beta \beta}$ e $\hat{\mathbf{K}}_{\beta \beta}^{0}$ são as matrizes de informação avaliadas em $\left(\hat{\boldsymbol{\beta}}^{T}, \hat{\sigma}^{2}, \hat{\boldsymbol{\rho}}^{T}\right)^{T} \mathrm{e}$ $\left(\hat{\beta}^{0^{T}}, \hat{\sigma}_{0}^{2},{\hat{\rho^{0}}}^{T}\right)^{T}$, respectivamente. Segue-se sob $H_{0}$ e para $n$ grande que $\xi_{R V}^{*}, \xi_{W}^{*}$ e $\xi_{S R}^{*}$ têm distribuição qui-quadrado com $k$ graus de liberdade.

\subsection{Restrições em desigualdade lineares}

O problema de maximização do logaritmo da função de verossimilhança do modelo (6.1) restrito ao conjunto de restrições em desigualdades lineares $\mathbf{C} \boldsymbol{\beta}$ $d \geqslant 0$ pode ser resolvido aplicando-se as condições de Kuhn-Tucker para atingir o máximo restrito. Para tal, considere a função Lagrangiana

$$
\mathcal{L}\left(\boldsymbol{\beta}, \sigma^{2}, \rho\right)=\mathrm{L}\left(\boldsymbol{\beta}, \sigma^{2}, \boldsymbol{\rho}\right)+\sum_{i=1}^{k} \lambda_{j}\left(\mathbf{C}_{j}^{T} \boldsymbol{\beta}-\mathrm{d}_{j}\right)
$$


em que $\lambda=\left(\lambda_{1}, \ldots, \lambda_{k}\right)^{T} \geqslant 0$ denota o vetor de multiplicadores de Lagrange. As condições suficientes para garantir que $\tilde{\boldsymbol{\beta}}$ corresponde às estimativa restrita por desigualdades (veja, por exemplo, Fahrmeir e Klinger, 1994) são dados por :

(i) $\mathrm{C}_{j}^{T} \tilde{\boldsymbol{\beta}}-\mathrm{d}_{j}=0$ para $j \in \mathrm{I} \subseteq\{1, \ldots, k\}$ e $\mathbf{C}_{j}^{T} \tilde{\boldsymbol{\beta}}>\mathrm{d}_{j}$ para todo $j \notin \mathrm{I}$; isto é, $\tilde{\boldsymbol{\beta}}$ é um ponto admissível;

(ii) existe $\tilde{\lambda}=\left(\tilde{\lambda}_{1}, \ldots, \tilde{\lambda}_{k}\right)^{T} \geqslant 0$ tal que $\partial \mathcal{L}\left(\boldsymbol{\beta}, \sigma^{2}, \rho\right) /\left.\partial \boldsymbol{\beta}\right|_{\left(\tilde{\beta}^{T}, \tilde{\theta}^{T}\right)^{T}}=0$; isto é, $\left(\tilde{\boldsymbol{\beta}}^{T}, \tilde{\boldsymbol{\theta}}^{T}\right)^{T}$ é um ponto estacionário;

(iii) $\left.\mathrm{s}^{T} \ddot{\mathrm{L}}\left(\beta, \sigma^{2}, \rho\right) \mathrm{s}\right|_{\left(\tilde{\beta}^{T}, \tilde{\theta}^{T}\right)^{T}}<0$ para todo $\mathrm{s} \neq 0$ e $\mathrm{s} \in\left\{\mathrm{s} \mid \mathrm{C}_{j}^{T} \mathrm{~s}-\mathrm{d}_{j}=0, j \in \mathrm{I}, \tilde{\lambda}_{j}>\right.$ 0 e $\left.\mathrm{C}_{j}^{T} \mathrm{~s}-\mathrm{d}_{j}>0, j \notin \mathrm{I}, \tilde{\lambda}_{j}=0\right\}$, em que $\ddot{\mathrm{L}}\left(\boldsymbol{\beta}, \sigma^{2}, \rho\right)$ denota a matriz de segundas derivadas com respeito a $\left(\boldsymbol{\beta}^{T}, \theta^{T}\right)^{T}$.

Essas condições são equivalentes a encontrar $\tilde{\boldsymbol{\beta}}$ através de um procedimento de busca que consiste em maximizar $\mathrm{L}\left(\boldsymbol{\beta}, \sigma^{2}, \boldsymbol{\rho}\right)$ sujeito a $\mathrm{C}_{j}^{T} \boldsymbol{\beta}-\mathrm{d}_{j}=0, j \in \mathrm{I}$, para cada $\mathbf{I} \subseteq\{1, \ldots, k\}$. A estimativa restrita por desigualdades $\tilde{\beta}$ é obtida de um problema de maximização que satisfaz as condições (i), (ii) e (iii). Assim, o problema restrito por desigualdades reduz a um problema restrito por igualdades que pode ser resolvido pelos procedimentos dados na Seção 6.3.

\subsection{Testes unilaterais}

Consideraremos nesta seção o problema de testar as hipóteses $\mathrm{H}_{0}: \mathbf{C} \boldsymbol{\beta}=\mathbf{d}$ contra $\mathrm{H}_{2}: \mathbf{C} \boldsymbol{\beta} \geqslant \mathrm{d}$, com pelo menos uma desigualdade estrita em $\mathrm{H}_{2}$. As estatísticas usuais razão de verossimilhanças, Wald e escore tomam, neste caso, as formas

$$
\begin{aligned}
\xi_{R V} & =2\left\{\mathrm{~L}\left(\tilde{\boldsymbol{\beta}}, \tilde{\sigma}^{2}, \tilde{\boldsymbol{\rho}}\right)-\mathrm{L}\left(\hat{\boldsymbol{\beta}}^{0}, \hat{\sigma}_{0}^{2}, \hat{\boldsymbol{\rho}}^{0}\right)\right\}, \\
\xi_{W} & =(\mathbf{C} \tilde{\boldsymbol{\beta}}-\mathbf{d})^{T}\left\{\mathbf{C} \tilde{\mathbf{K}}_{\beta \beta}^{-1} \mathrm{C}^{T}\right\}^{-1}(\mathbf{C} \tilde{\boldsymbol{\beta}}-\mathrm{d}) \mathrm{e} \\
\xi_{S R} & =\left\{\mathrm{U}_{\boldsymbol{\beta}}\left(\hat{\boldsymbol{\beta}}^{0}, \hat{\sigma}_{0}^{2}, \hat{\boldsymbol{\rho}}^{0}\right)-\mathrm{U}_{\boldsymbol{\beta}}\left(\tilde{\boldsymbol{\beta}}, \tilde{\sigma}^{2}, \tilde{\boldsymbol{\rho}}\right)\right\}^{T}\left(\hat{\mathbf{K}}_{\beta \beta}^{0}\right)^{-1}\left\{\mathrm{U}_{\beta}\left(\hat{\boldsymbol{\beta}}^{0}, \hat{\sigma}_{0}^{2}, \hat{\boldsymbol{\rho}}^{0}\right)-\mathrm{U}_{\beta}\left(\tilde{\boldsymbol{\beta}}, \tilde{\sigma}^{2}, \tilde{\boldsymbol{\rho}}\right)\right\},
\end{aligned}
$$

respectivamente. Além disso, suponha que o espaço paramétrico de $\beta$ é um aberto. Isso quer dizer que, embora exista interesse em testar na direção unilateral $\mathbf{C} \boldsymbol{\beta} \geqslant \mathrm{d}$, a direção complementar não está descartada teoricamente. Sob certas condições de regularidade, segue de Gouriéroux e Monfort (1995, Seção 21.3) que as estatísticas 
$\xi_{R V}, \xi_{W}$ e $\xi_{S R}$ são, assintoticamente equivalentes a uma mistura de distribuições qui-quadrados, dada por

$$
\operatorname{Pr}\left\{\xi_{R V} \geqslant c\right\}=\sum_{\ell=0}^{k} \omega(k, \ell ; \Delta) \operatorname{Pr}\left\{\chi_{\ell}^{2} \geqslant c\right\}
$$

em que $c \geqslant 0, \Delta=\mathrm{CK}_{\beta \beta}^{-1} \mathrm{C}^{T}, \chi_{0}^{2}$ denota a distribuição degenerada na origem, $\mathrm{e}$ $\omega(k, \ell ; \Delta)$ 's são pesos conhecidos como probabilidades de nível, que são expressos como funções dos coeficientes de correlação associados com a matriz $\Delta$. Pode-se mostrar, facilmente, que os coeficientes de correlação associados com a matriz $\Delta$ não dependem, neste caso, de $\boldsymbol{\beta}$, mas podem depender dos coeficientes de correlação $\left(\rho_{1}, \ldots, \rho_{q}\right)^{T}$. Assim, deveremos procurar pela situação menos favorável no conjunto $\rho=\left(\rho_{1}, \ldots, \rho_{q}\right)^{T}$ de modo que (6.9) ficará expresso na forma

$$
\operatorname{Pr}\left\{\xi_{R V} \geqslant c\right\}=\sup _{\Delta} \sum_{\ell=0}^{k} \omega(k, \ell ; \Delta) \operatorname{Pr}\left\{\chi_{\ell}^{2} \geqslant c\right\}
$$

ou então deve-se substituir $\Delta(\rho)$ em (6.9) por uma estimativa consistente.

\subsection{Ordem simples}

Considere novamente o caso de $g$ grupos discutido na Seção 6.2 e o caso de testar ordem simples $\mathrm{H}_{0}: \mu_{1}=\cdots=\mu_{g}$ contra $\mathrm{H}_{2}: \mu_{1} \leqslant \cdots \leqslant \mu_{g}$, com pelo menos uma desigualdade estrita em $\mathrm{H}_{2}$. Por exemplo, a estatística Wald assume aqui a seguinte forma :

$$
\xi_{W}=(\mathbf{C} \tilde{\mu})^{T}\left(\mathbf{C}^{T} \tilde{\mathbf{V}}_{\mu} \mathbf{C}\right)^{-1}(\mathbf{C} \tilde{\mu})
$$

em que $\mathrm{C}$ é uma matriz $(g-1) \times g$ cuja $j$-ésima linha é formada por zeros com um na $(j+1)$-ésima posição e -1 na $j$-ésima posição, $\mathrm{V}_{\mu}=\operatorname{diag}\left\{v_{1}^{2}, \ldots, v_{g}^{2}\right\}, \tilde{\mu}$ é a estimativa de máxima verossimilhança de $\boldsymbol{\mu}$ sob $\mathrm{H}_{2}$. Pode-se mostrar que as probabilidades de nível $\omega(0, g-1 ; \Delta), \ldots, \omega(g-1, g-1 ; \Delta)$ não dependem de $\rho$ 
neste caso. As correlações associadas com a matriz $\Delta$ assumem a forma

$$
\begin{aligned}
\varrho_{\ell(\ell+1)}=\varrho_{(\ell+1) \ell} & =-\left[\frac{v_{\ell}^{-2} v_{j+2}^{-2}}{\left(v_{\ell+1}^{-2}+v_{\ell}^{-2}\right)\left(v_{j+1}^{-2}+v_{j+2}^{-2}\right)}\right]^{1 / 2} \\
& =-\left[\frac{\gamma_{\ell}^{-2} \gamma_{j+2}^{-2}}{\left(\gamma_{\ell+1}^{-2}+\gamma_{\ell}^{-2}\right)\left(\gamma_{j+1}^{-2}+\gamma_{j+2}^{-2}\right)}\right]^{1 / 2},
\end{aligned}
$$

$\ell=1, \ldots, g-2$ e $\varrho_{\ell \ell^{\prime}}=0$ para $\left|\ell-\ell^{\prime}\right|>1$, e não dependem de $\mu$. Assim, a distribuição nula assintótica do lado direito de (6.9) é única para o caso de ordem simples . Outros casos usuais em que essa distribuição nula assintótica é também única são de árvore simples e de ordem unimodal (veja, por exemplo, Robertson, Wright e Dykstra, Cap. 3, 1998; Paula e Sen, 1995).

\subsection{Estudos de simulação}

A fim de estudar o comportamento do poder das estatísticas $\xi_{R V}$, $\xi_{W}$ e $\xi_{S R}$ em amostras pequenas e moderadas, e para a mesma estrutura de correlação para $\mathbf{R}(\rho)$, como também o ganho de poder de $\xi_{R V}, \xi_{W}$ e $\xi_{S R}$ sobre $\xi_{R V}^{*}$, $\xi_{W}^{*}$ e $\xi_{S R}^{*}$, respectivamente, realizamos vários estudos de simulação, supondo dados agrupados e sob a presença de regressores. Dois tipos de estruturas de correlação, uniforme e $\mathrm{AR}(1)$, entre as respostas do mesmo indivíduo foram assumidos para $\mathbf{R}(\rho)$ e foram considerados dois parâmetros restritos. Os valores da correlação $\rho$ usados no estudo variam no intervalo $[0 ; 0,8]$. Para cada configuração paramétrica, 5000 amostras simuladas foram avaliadas. Um programa em Ox foi desenvolvido para executar o estudo de simulação.

O poder foi estimado como a proporção de $p$-valores maiores do que o nível de significância $\alpha$. Para as estatísticas $\xi_{R V}^{*}, \xi_{W}^{*}$ e $\xi_{S R}^{*}$ o $p$-valor é definido como $\mathrm{P}=\operatorname{Pr}\left\{\chi_{2}^{2} \geqslant t\right\}$, em que $t$ é o valor da estatística do teste, enquanto que para $\xi_{R V}$, $\xi_{W}$ e $\xi_{S R}$ o $p$-valor é definido para $t>0$ como

$$
\mathrm{P}=\sup _{\Delta}\left\{\omega(2,1 ; \Delta) \operatorname{Pr}\left(\chi_{1}^{2} \geqslant t\right)+\omega(2,2 ; \Delta) \operatorname{Pr}\left(\chi_{2}^{2} \geqslant t\right)\right\}
$$

e $\mathrm{P}=1$ para $t=0$. Os pesos neste caso são dados por $\omega(2,1 ; \Delta)=\frac{1}{2}$ e $\omega(2,2 ; \Delta)=$ 
ESTUDOS DE SIMULAÇÃO

$\frac{1}{2}-\frac{1}{2} \pi^{-1} \cos ^{-1}(\varrho)$ com $\varrho$ denotando o coeficiente de correlação associado com a matriz $\Delta$.

\subsubsection{Dados agrupados}

\section{Caso I}

Seja $\mathbf{y}_{i}$ uma variável aleatória distribuída segundo uma distribuição $t$-multivariada $t_{m}(\boldsymbol{\mu}, \Phi, \nu)$, em que $m=3, \mu=\left(\mu_{1}, \mu_{2}, \mu_{3}\right)^{T}$ e $\Phi=\sigma^{2} \mathbf{R}(\rho)$, para $i=$ $1, \ldots, n$. Os valores de $\nu$ e $\sigma^{2}$ foram fixadas como sendo $\nu=3$ e $\sigma^{2}=2$, e o nível nominal foi $\alpha=0,05$. Foram geradas amostras para tamanhos amostrais de $n=20,50$ e 100. O interesse aqui é testar as hipóteses $\mathrm{H}_{0}: \mu_{1}=\mu_{2}=\mu_{3}$ contra $\mathrm{H}_{2}: \mu_{1} \leqslant \mu_{2} \leqslant \mu_{3}$, com pelo menos uma desigualdade estrita em $\mathrm{H}_{2}$. Nos dados gerados trabalhamos com $\mu_{1}=\mu_{2}=1$ e $\mu_{3}=1+\delta$, em que $\delta=0 ; 0,2 ; 0,4 ; 0,6$ e 0,8 . A matriz $\Delta$ toma aqui a forma

$$
\Delta=\frac{\sigma^{2}}{n}\left(\frac{\nu+m+2}{\nu+m}\right)\left[\begin{array}{cc}
2-2 \rho_{1} & \rho_{1}-\rho_{2}+\rho_{3}-1 \\
\rho_{1}-\rho_{2}+\rho_{3}-1 & 2-2 \rho_{3}
\end{array}\right]
$$

em que $\rho_{1}=\rho_{3}=\rho$ e $\rho_{2}=\rho^{2}$, consequentemente $\varrho=(\rho-1) / 2$ para a estrutura $\operatorname{AR}(1) ; \rho_{1}=\rho_{2}=\rho_{3}=\rho$, de modo que $\varrho=-0,5$ para a estrutura uniforme. Assim, a região menos favorável ocorre quando $\rho=1$ para $\mathrm{AR}(1), \log \mathrm{P}=$ $\frac{1}{2} \operatorname{Pr}\left\{\chi_{1}^{2} \geqslant t\right\}+\frac{1}{4} \operatorname{Pr}\left\{\chi_{2}^{2} \geqslant t\right\}$ e é unicamente determinada para estrutura uniforme com $\mathrm{P}=\frac{1}{2} \operatorname{Pr}\left\{\chi_{1}^{2} \geqslant t\right\}+\frac{1}{6} \operatorname{Pr}\left\{\chi_{2}^{2} \geqslant t\right\}, t>0$.

Figura 6.1 Distribuições acumuladas teórica e empírica da estatística $\xi_{R V}$ para dados agrupados (caso I), $n=20$ e para a estrutura $\mathrm{AR}(1)$.
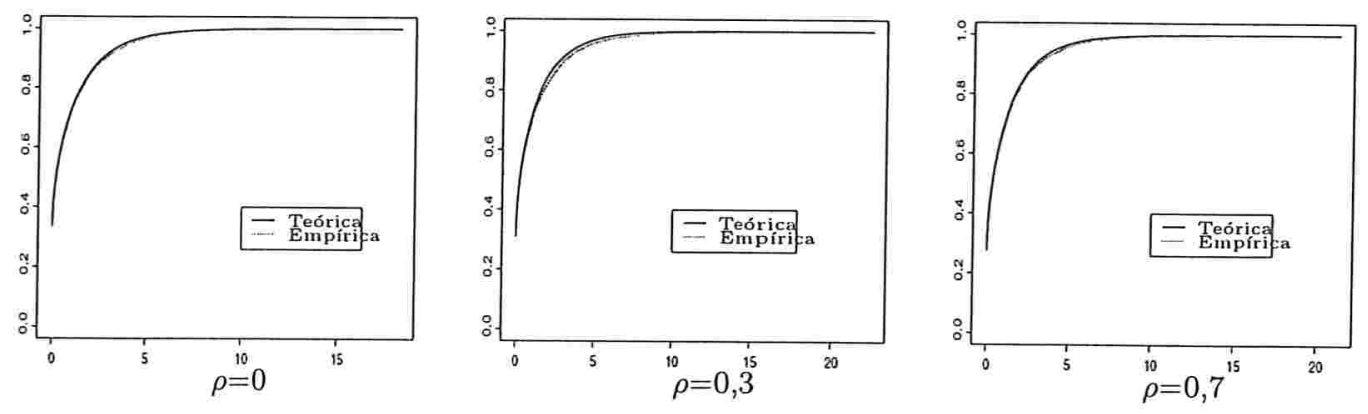
ESTUDOS DE SIMULAÇÃO

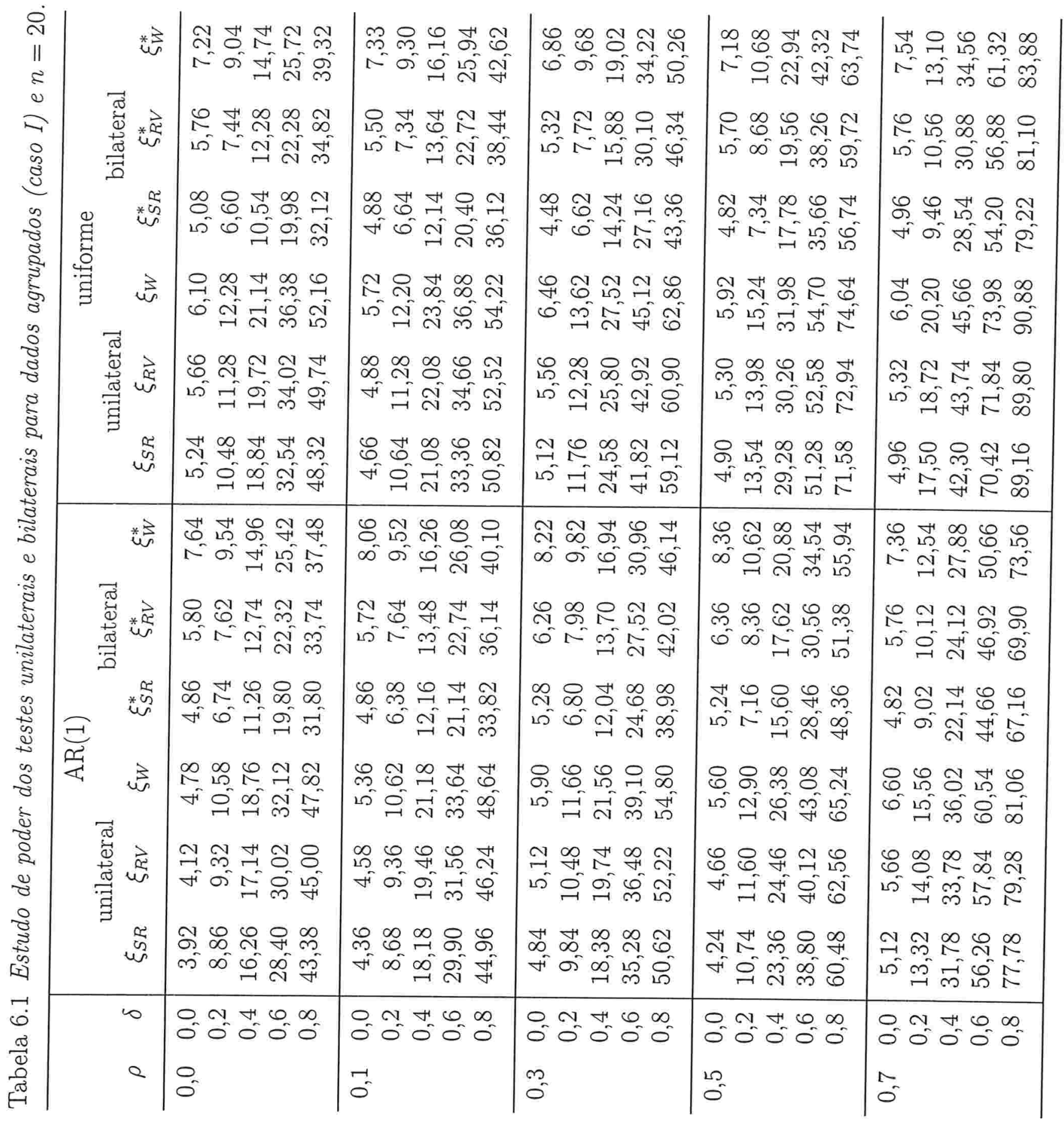




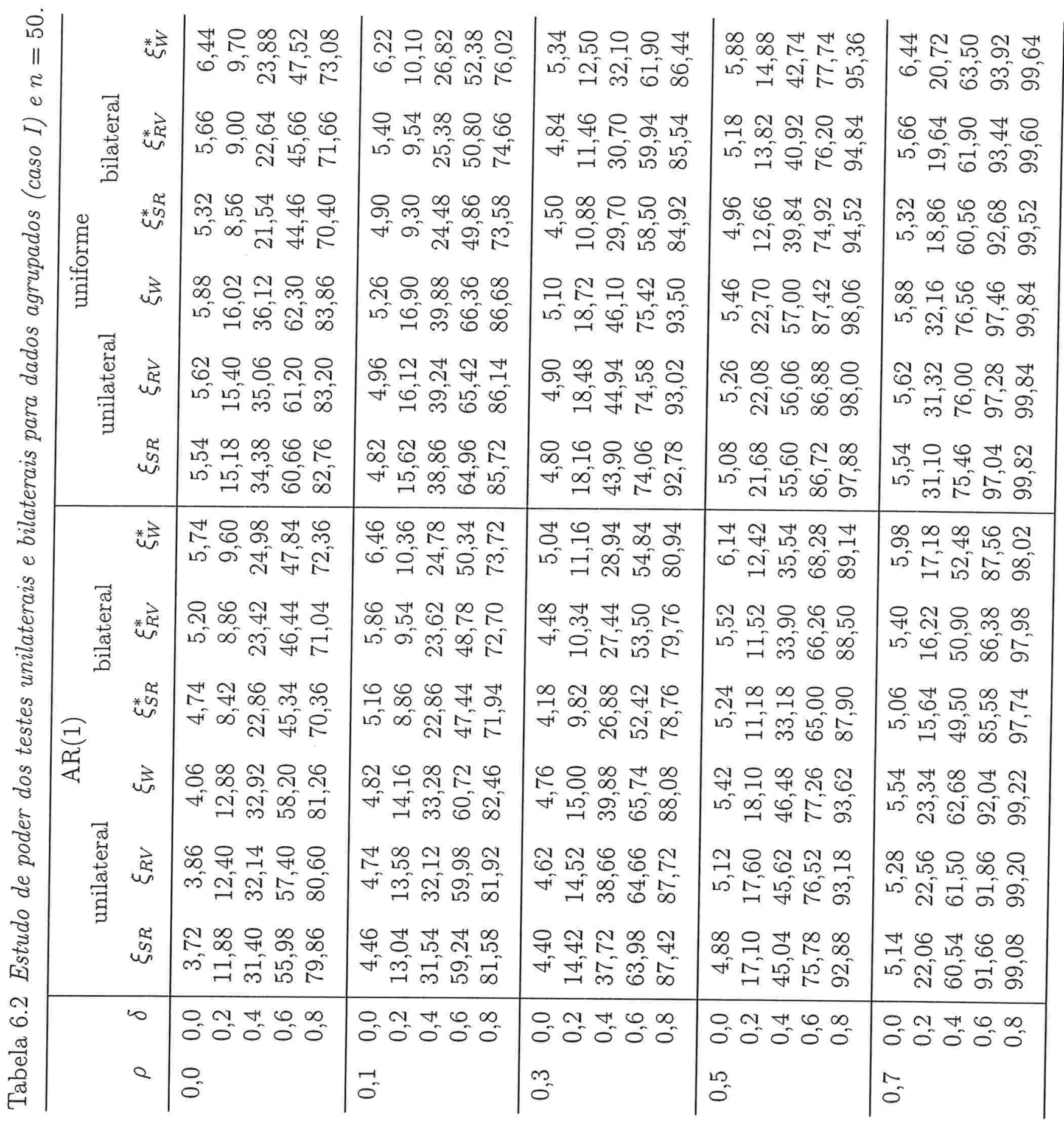




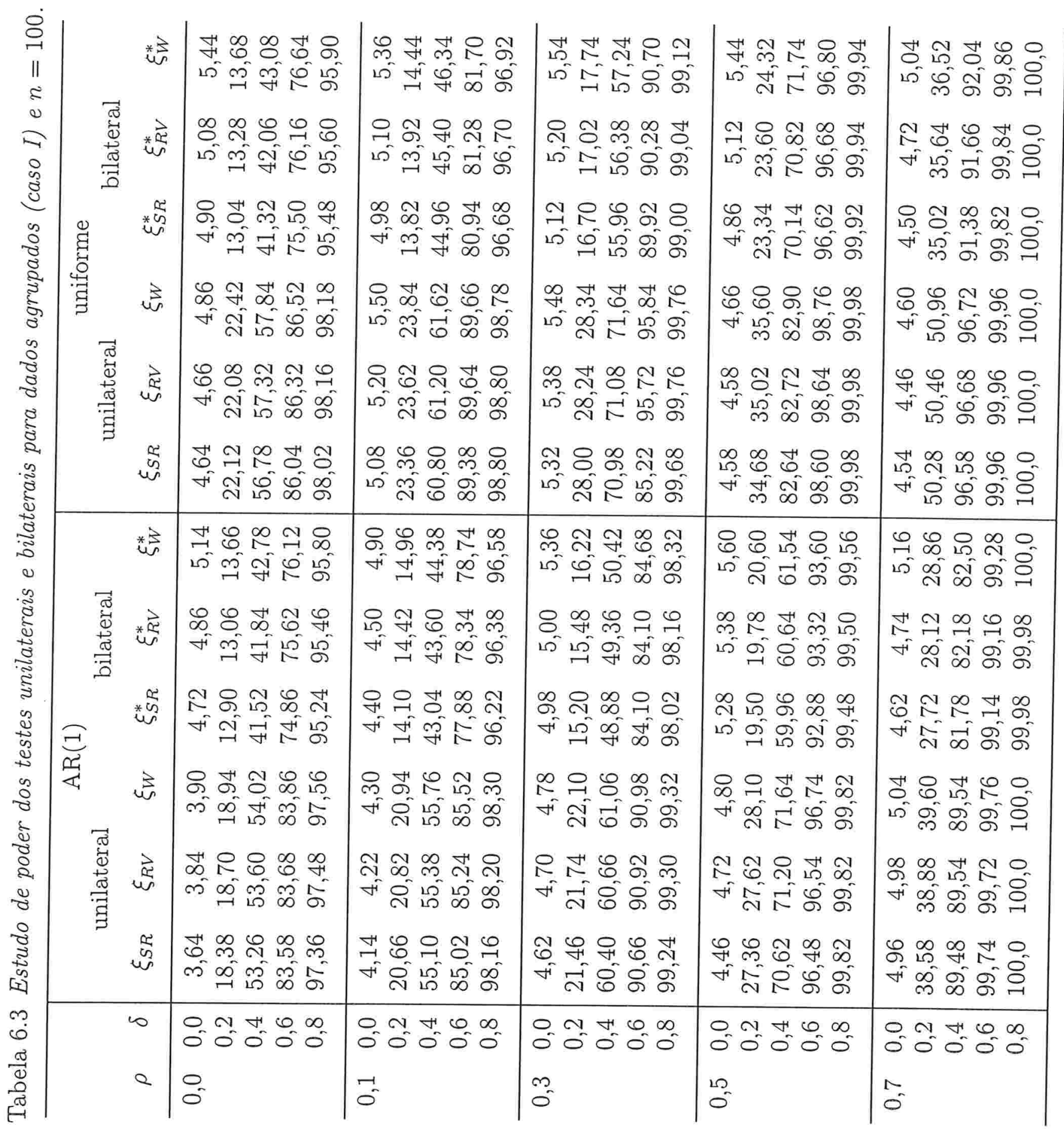


Figura 6.2 Distribuições acumuladas teórica e empírica da estatística $\xi_{R V}$ para dados agrupados (caso I), $n=20$ e para a estrutura uniforme.
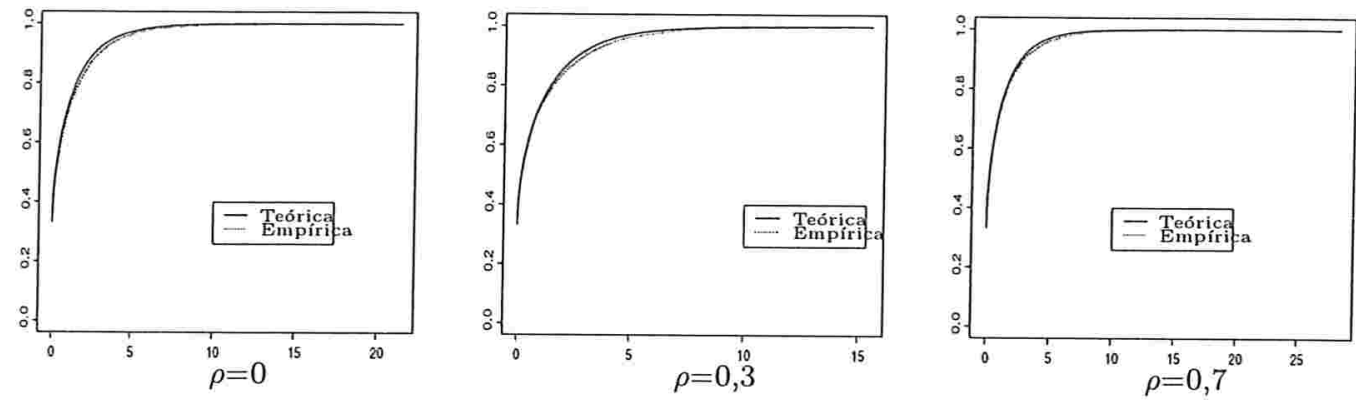

Figura 6.3 Distribuições acumuladas teórica e empírica da estatística $\xi_{R V}$ para dados agrupados (caso I), $n=50$ e para a estrutura $\mathrm{AR}(1)$.
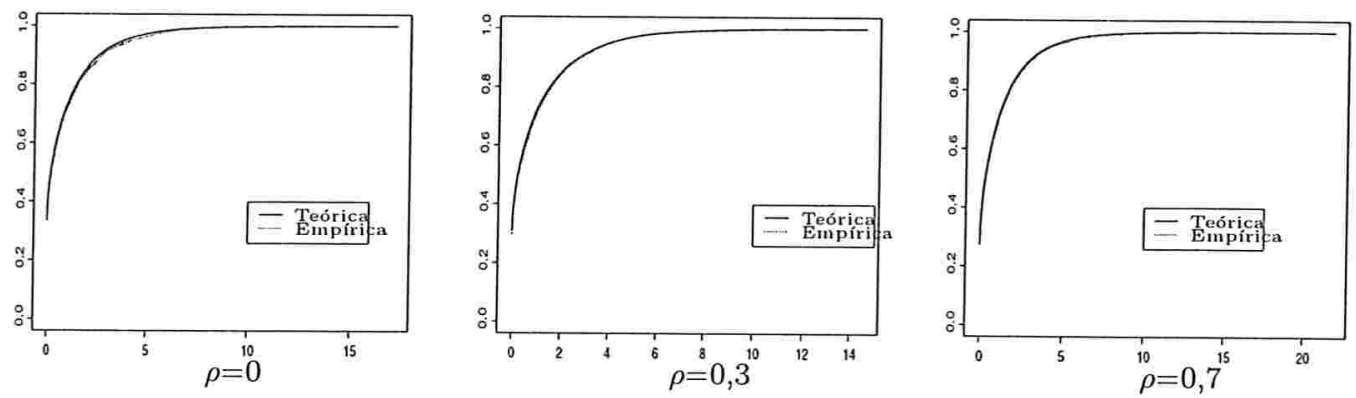

Figura 6.4 Distribuições acumuladas teórica e empírica da estatística $\xi_{R V}$ para dados agrupados (caso I), $n=50$ e para a estrutura uniforme.
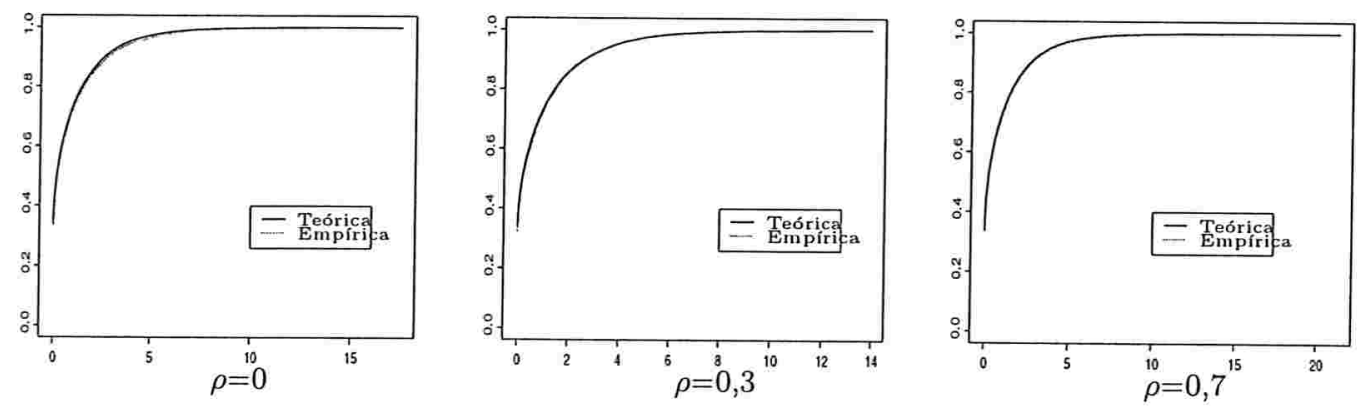
Tabela 6.4 Diferenças (em valor absoluto) entre a distribuição acumulada teórica e empírica da estatística $\xi_{R V}$ para dados agrupados (caso I) (em \%).

\begin{tabular}{|c|c|c|c|c|c|c|c|c|c|c|c|c|c|}
\hline & \multirow[b]{2}{*}{$\rho$} & \multicolumn{6}{|c|}{$\begin{array}{c}\mathrm{AR}(1) \\
\text { percentil teórico }\end{array}$} & \multicolumn{6}{|c|}{$\begin{array}{l}\text { uniforme } \\
\text { percentil teórico }\end{array}$} \\
\hline & & $25 \%$ & $50 \%$ & $75 \%$ & $90 \%$ & $95 \%$ & $\max$ & $25 \%$ & $50 \%$ & $75 \%$ & $90 \%$ & $95 \%$ & $\max$ \\
\hline \multirow[t]{3}{*}{$n=20$} & 0,0 & 0,1 & 0,4 & 0,6 & 0,8 & 0,9 & 1,0 & 1,1 & 1,8 & 2,2 & 2,3 & 2,4 & 2,6 \\
\hline & 0,3 & 0,4 & 0,8 & 1,4 & 2,2 & 2,3 & 2,5 & 0,2 & 0,4 & 1,2 & 1,5 & 1,6 & 1,9 \\
\hline & 0,7 & 0,3 & 0,5 & 0,7 & 0,9 & 1,0 & 1,2 & 0,2 & 0,5 & 0,9 & 1,3 & 1,4 & 1,5 \\
\hline \multirow[t]{3}{*}{$n=50$} & 0,0 & 0,3 & 0,7 & 1,0 & 1,2 & 1,2 & 1,5 & 0,4 & 0,6 & 0,8 & 1,0 & 1,1 & 1,4 \\
\hline & 0,3 & 0,3 & 0,7 & 1,0 & 1,3 & 1,4 & 1,7 & 0,2 & 0,6 & 0,9 & 1,2 & 1,3 & 1,6 \\
\hline & 0,7 & 0,2 & 0,3 & 0,5 & 0,9 & 1,0 & 1,2 & 0,2 & 0,3 & 0,4 & 0,6 & 0,7 & 0,8 \\
\hline
\end{tabular}

Os resultados do estudo de simulação são apresentados nas Tabelas 6.1-6.3. Notase que os tamanhos empíricos dos testes tendem ao nível nominal, à medida que os tamanhos da amostra crescem. O desvio padrão da estimativa do poder é limitado por $1 / \sqrt{20000}$. O poder aumenta à medida que o tamanho amostral e a correlação aumentam. Essa última tendência é esperada pois, quanto maior é a correlação entre $y_{i j}$ e $y_{i j}, j \neq j^{\prime}$, mais alta tende a ser a probabilidade do teste detectar diferenças entre os valores médios $\mu_{j}$ e $\mu_{j^{\prime}}$, se eles são diferentes. Podemos também notar a superioridade dos testes unilaterais sobre os testes bilaterais. As Figuras 6.1-6.4 exibem as distribuições nulas teórica e empírica da estatística $\xi_{R V}$. Em geral, podemos notar uma boa concordância entre as duas distribuições. Pequenas diferenças aparecem para o caso de $n=20$. Comportamento similar foi encontrado para as estatísticas $\xi_{S R}$ e $\xi_{W}$. A Tabela 6.4 apresenta as diferenças entre as distribuições nulas teórica e empírica da estatística $\xi_{R V}$ para alguns percentis da distribuição nula assintótica, como pode-se notar os valores máximos são em geral menores do que $2 \%$ mesmo para $n=20$. Além disso, é interessante notar que as diferenças parecem decrescer, à medida que a correlação cresce. 
Caso II

Seja $y_{i \ell}$ uma variável aleatória distribuída segundo uma distribuição $t$-multivariada $t_{m}\left(\mu_{\ell}, \Phi, \nu\right)$, em que $m=4, \mu_{\ell}=\mu_{\ell} 1_{m}$ e $\Phi=\sigma^{2} \mathbf{R}(\rho)$, para $i=1, \ldots, n_{\ell}$ e $\ell=1,2,3$. Os valores de $\nu$ e $\sigma^{2}$ foram também fixados com valores $\nu=3$ e $\sigma^{2}=1$. Os tamanhos dos grupos foram os mesmos, sendo $n_{1}=n_{2}=n_{3}=10,20$ e 50 . O interesse aqui é testar as hipóteses $\mathrm{H}_{0}: \mu_{1}=\mu_{2}=\mu_{3}$ contra $\mathrm{H}_{2}: \mu_{1} \leqslant \mu_{2} \leqslant \mu_{3}$, com pelo menos uma desigualdade estrita em $\mathrm{H}_{2}$. Trabalhamos com o mesmo conjunto de valores para $\mu_{1}, \mu_{2}$ e $\mu_{3}$ assumidos no caso I. A matriz $\Delta$ assume aqui a forma

$$
\Delta=\frac{\sigma^{2}}{n}\left(\frac{\nu+m+2}{\nu+m}\right) \frac{1}{\mathbf{1}^{T} \operatorname{vec}\left(\mathbf{R}^{-1}\right)}\left(\begin{array}{rr}
2 & -1 \\
-1 & 2
\end{array}\right)
$$

A situação menos favorável é única para ambas as estruturas em que $\varrho=-0,5$. Encontramos $\mathrm{P}=\frac{1}{2} \operatorname{Pr}\left\{\chi_{1}^{2} \geqslant t\right\}+\frac{1}{6} \operatorname{Pr}\left\{\chi_{2}^{2} \geqslant t\right\}, t>0$ para ambos os casos.

Os resultados do estudo de simulação são descritos nas Tabelas 6.5-6.7. Note que, à medida que $n$ cresce, os tamanhos empíricos dos testes ficam mais próximos ao nível nominal. O poder cresce quando o tamanho da amostra cresce, porém, contrário ao caso I, o poder diminui à medida que a correlação cresce. Essa última tendência é também esperada, uma vez que as observações $y_{i \ell 1}, \ldots, y_{i \ell m}$ não fornecem muita informação sobre $\mu_{\ell}$ se elas são altamente correlacionados. É evidente a superioridade de $\xi$ sobre $\xi^{*}$, particularmente próximo à hipótese nula. As Figuras 6.5-6.8 exibem as distribuições nulas teórica e empírica da estatística $\xi_{R V}$. Podemos também notar uma boa concordância entre as duas distribuições. Contudo, a distribuição empírica parece subestimar a distribuição nula teórica para réplicas de tamanhos iguais a 10 . Comportamento similar foi encontrado para as estatísticas restantes. A Tabela 6.8 exibe as diferenças entre as distribuições nulas teórica e empírica da estatística $\xi_{R V}$ para alguns percentis da distribuição nula assintótica. Podemos observar nesta tabela que as diferenças máximas são, em geral, não maiores do que $2 \%$, e as mesmas, parecem crescer à medida que a correlação cresce. 
ESTUDOS DE SIMULAÇÃO

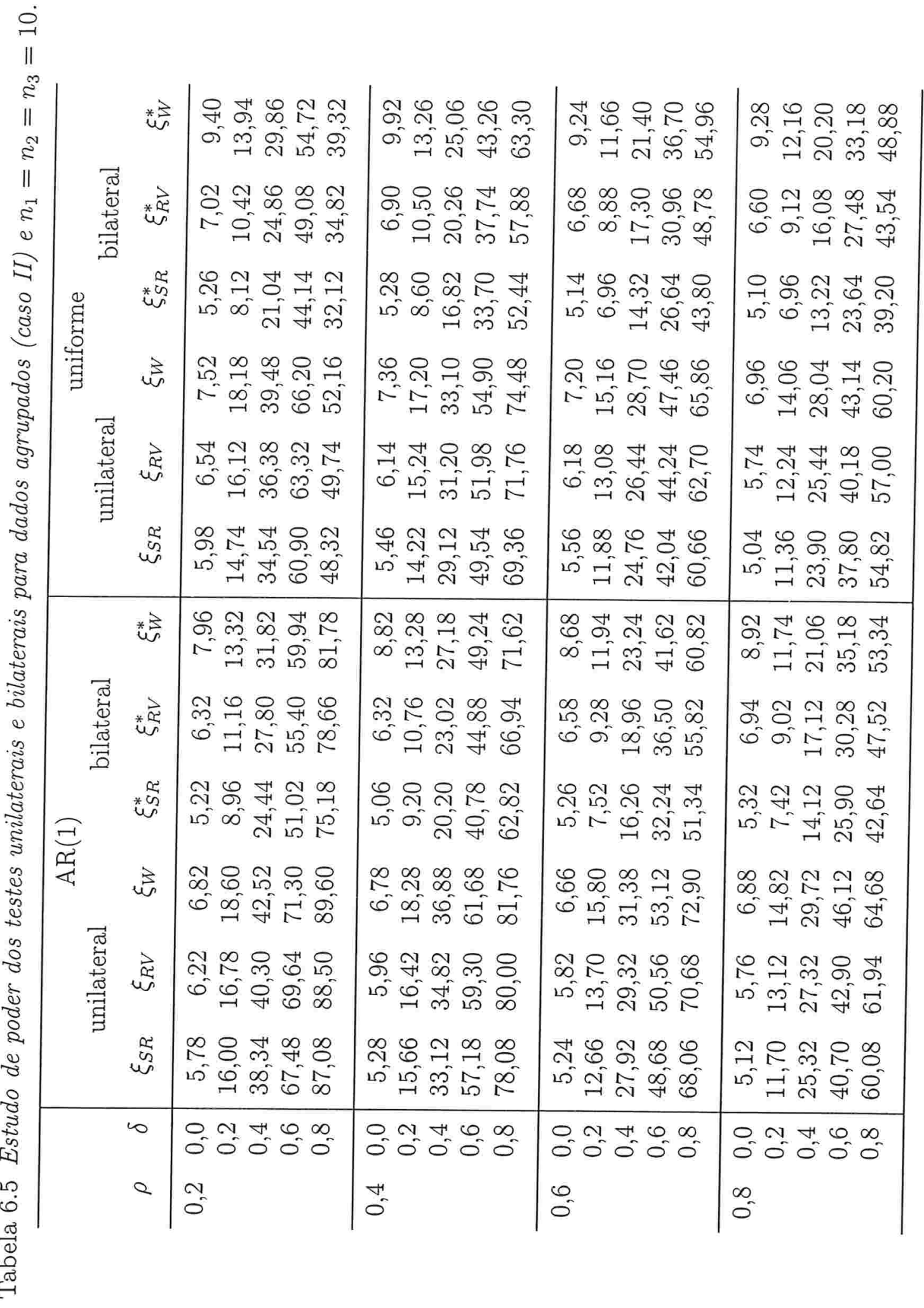




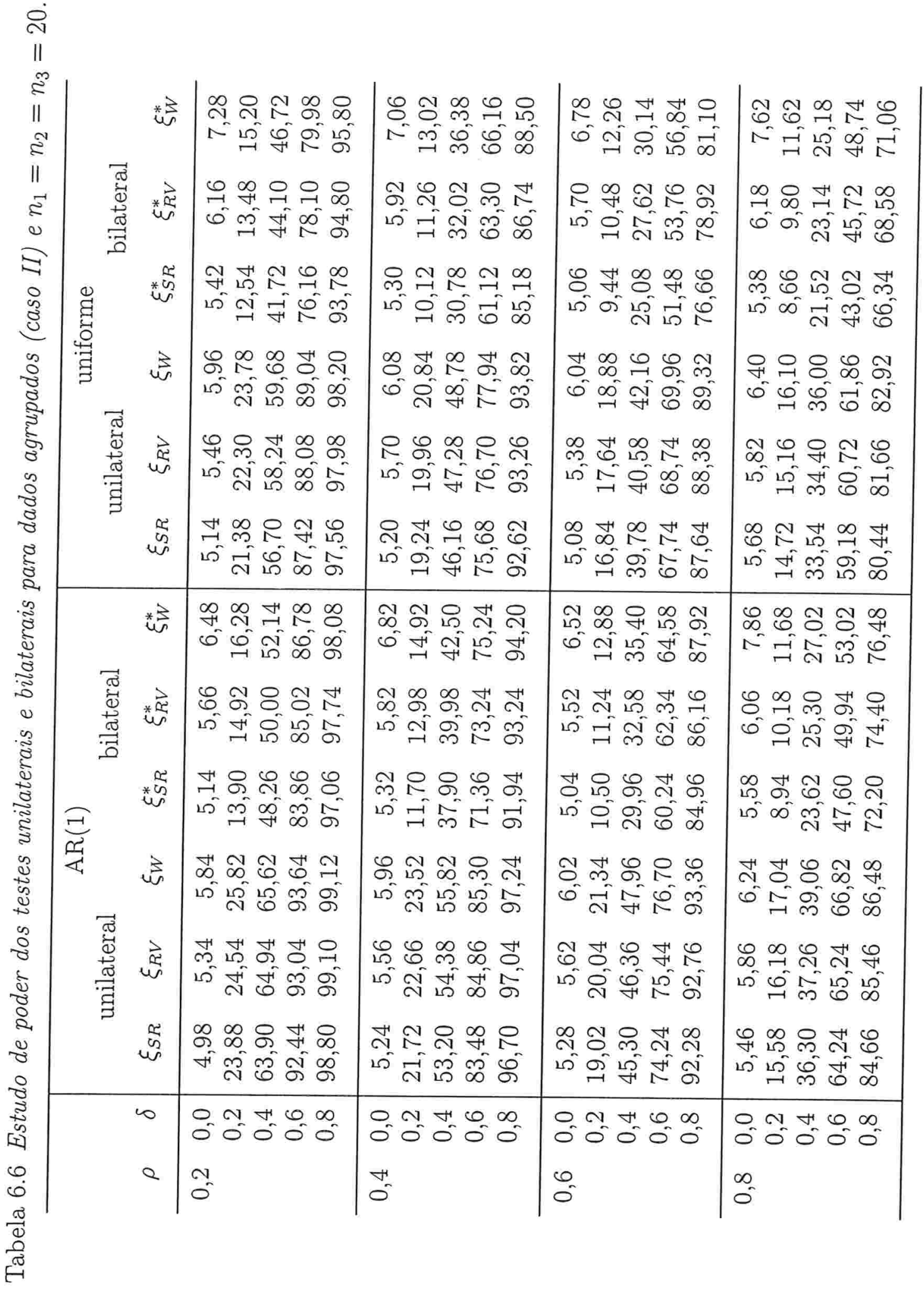




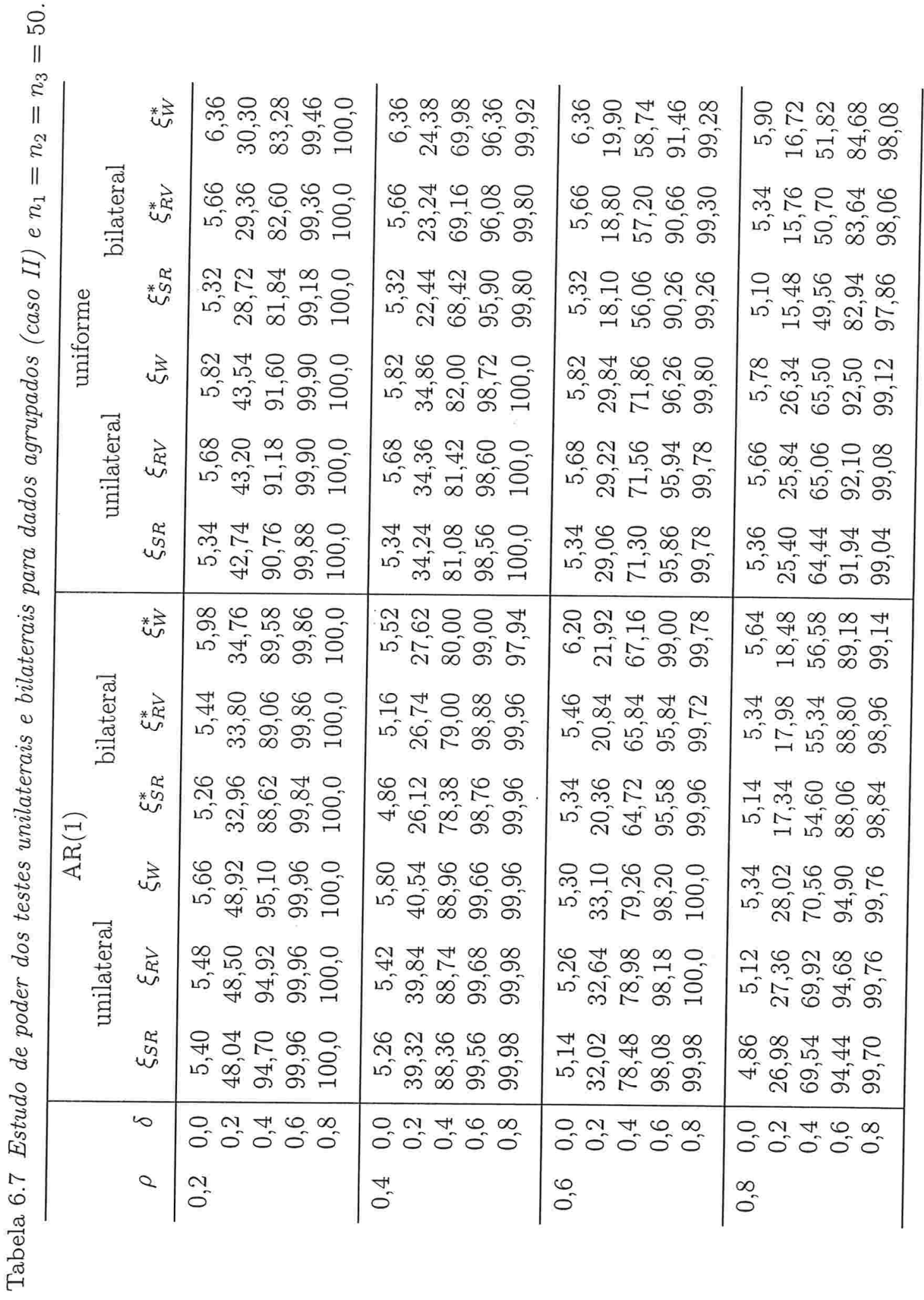


Figura 6.5 Distribuições acumuladas teórica e empírica da estatística $\xi_{R V}$ para dados agrupados (caso II), $n_{1}=n_{2}=n_{3}=10$ e para estrutura $\mathrm{AR}(1)$.
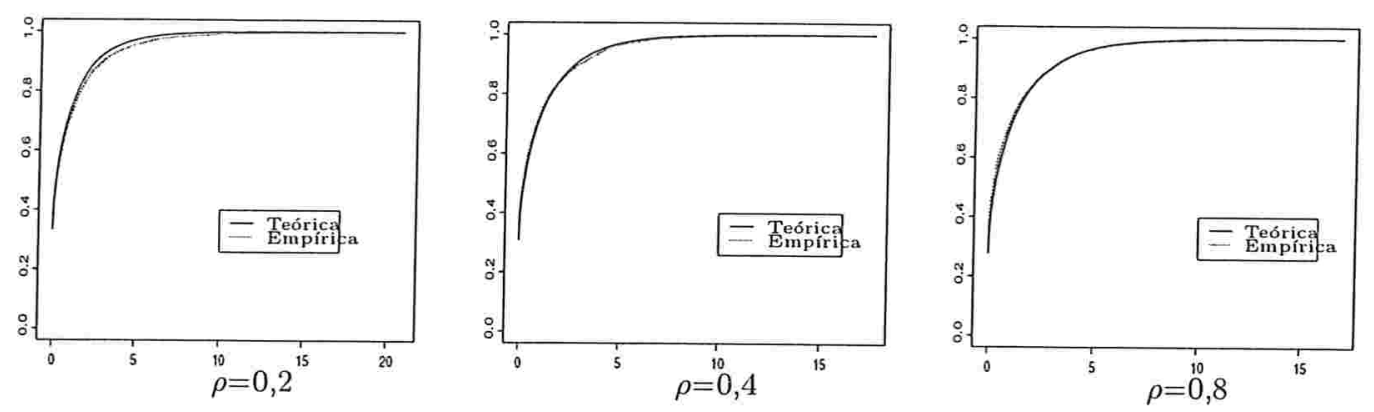

Figura 6.6 Distribuições acumuladas teórica e empírica da estatística $\xi_{R V}$ para dados agrupados (caso II), $n_{1}=n_{2}=n_{3}=10$ e para estrutura uniforme.
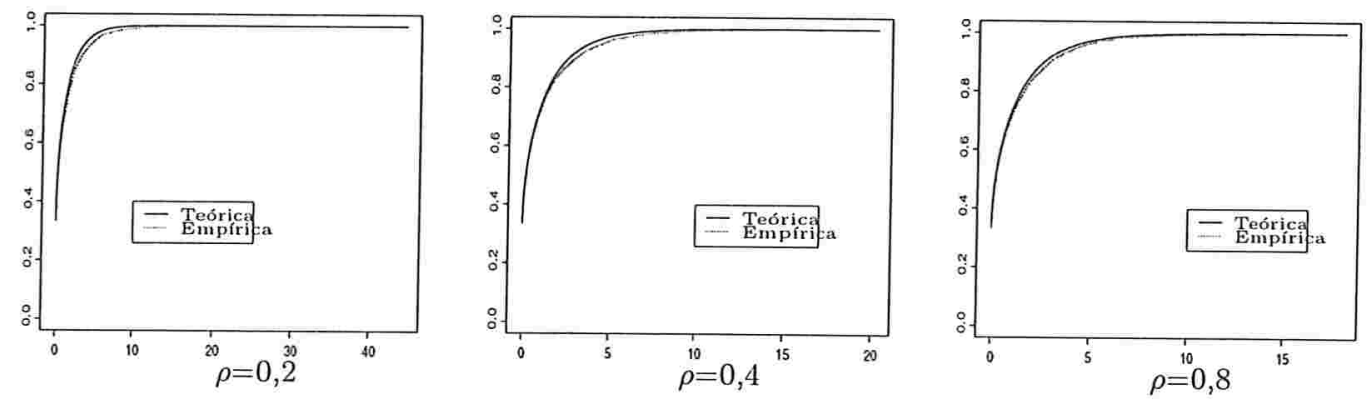

Figura 6.7 Distribuições acumuladas teórica e empírica da estatística $\xi_{R V}$ para dados agrupados (caso II), $n_{1}=n_{2}=n_{3}=20$ e para estrutura $\operatorname{AR}(1)$.
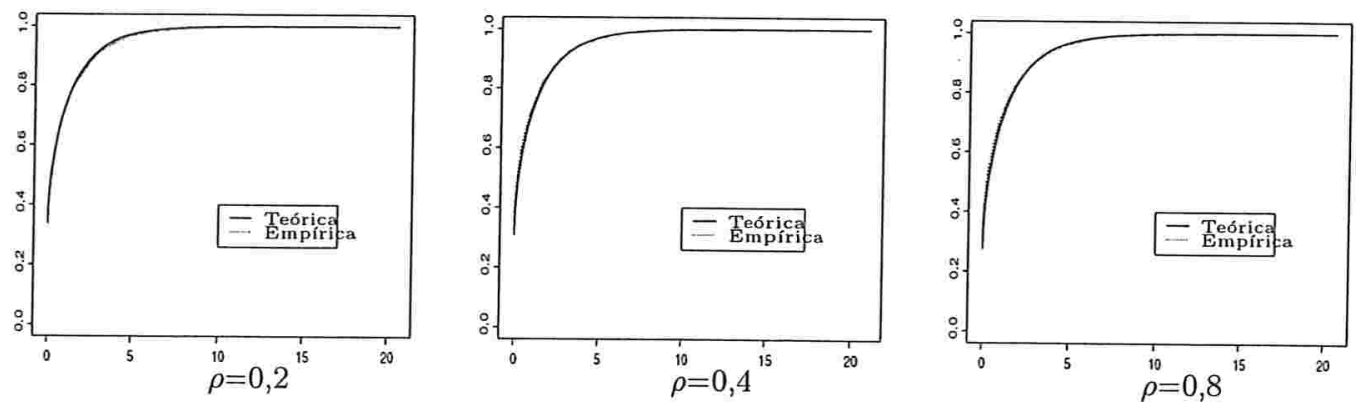
Figura 6.8 Distribuições acumuladas teórica e empírica da estatística $\xi_{R V}$ para dados agrupados (caso II), $n_{1}=n_{2}=n_{3}=20$ e para estrutura uniforme.
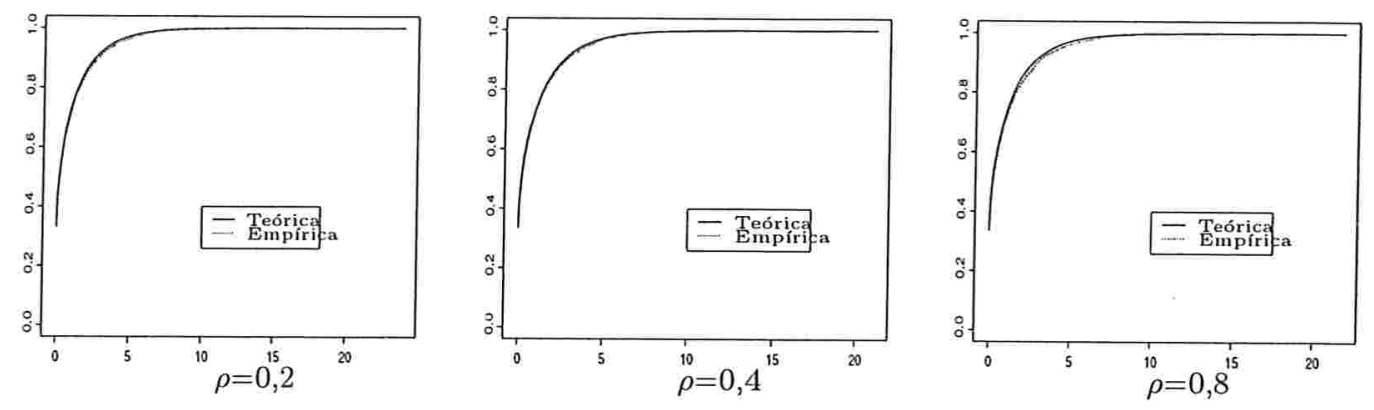

Tabela 6.8 Diferenças (em valor absoluto) entre a distribuição acumulada teórica e empírica da estatística $\xi_{R V}$ para dados agrupados (caso II) (em \%).

\begin{tabular}{|c|c|c|c|c|c|c|c|c|c|c|c|c|c|}
\hline & \multirow[b]{2}{*}{$\rho$} & \multicolumn{6}{|c|}{$\begin{array}{c}\mathrm{AR}(1) \\
\text { percentil teórico }\end{array}$} & \multicolumn{6}{|c|}{$\begin{array}{c}\text { uniforme } \\
\text { percentil teórico }\end{array}$} \\
\hline & & $25 \%$ & $50 \%$ & $75 \%$ & $90 \%$ & $95 \%$ & $\max$ & $25 \%$ & $50 \%$ & $75 \%$ & $90 \%$ & $95 \%$ & $\max$ \\
\hline \multirow[t]{3}{*}{$n_{i}=10$} & 0,2 & 1,0 & 1,7 & 2,1 & 2,3 & 2,4 & 2,6 & 1,3 & 2,1 & 2,8 & 3,0 & 3,1 & 3,4 \\
\hline & 0,4 & 0,2 & 0,3 & 0,7 & 1,4 & 1,6 & 2,0 & 0,3 & 0,5 & 1,2 & 1,8 & 1,9 & 2,2 \\
\hline & 0,8 & 0,3 & 0,8 & 1,4 & 1,6 & 1,8 & 2,0 & 0,2 & 1,1 & 1,6 & 1,8 & 2,0 & 2,2 \\
\hline \multirow[t]{3}{*}{$n_{i}=20$} & 0,2 & 0,1 & 0,2 & 0,4 & 0,8 & 0,9 & 1,2 & 0,2 & 0,5 & 0,8 & 1,1 & 1,3 & 1,3 \\
\hline & 0,4 & 0,3 & 0,7 & 1,2 & 1,4 & 1,5 & 1,8 & 0,5 & 0,8 & 1,0 & 1,4 & 1,5 & 1,7 \\
\hline & 0,8 & 0,4 & 1,2 & 1,5 & 1,6 & 1,7 & 1,8 & 0,2 & 0,9 & 1,4 & 1,8 & 1,9 & 2,1 \\
\hline
\end{tabular}

\subsubsection{Presença de regressores}

Assumiremos aqui que $\mathrm{y}_{i}$ segue distribuição $t$-multivariada $t_{m}\left(\mu_{i}, \Phi, \nu\right)$, em que $m=4, \Phi=\sigma^{2} \mathbf{R}(\rho)$ e $\boldsymbol{\mu}_{i}=\mu_{i} \mathbf{1}_{m}$, com

$$
\mu_{i}=\beta_{0}+\beta_{1} x_{1 i}+\beta_{2} x_{2 i}
$$

para $i=1, \ldots, n$. Os valores de $x_{1 i}$ e $x_{2 i}$ foram gerados de uma distribuição uniforme no intervalo $[0 ; 1]$. Os valores dos parâmetros foram fixados em $\beta_{0}=\beta_{1}=$ $\beta_{2}=1, \nu=3$ e $\sigma^{2}=2$. Os tamanhos amostrais usados foram $n=20,50$ e 100 . O interesse aqui é testar as hipóteses $H_{0}: \beta_{1}=1, \beta_{2}=1$, contra $H_{2}: \beta_{1} \geqslant 1, \beta_{2} \geqslant 1$, 
com pelo menos uma desigualdade estrita em $\mathrm{H}_{2}$. Na alternativa trabalhamos com os valores dos parâmetros $\beta_{1}=1+\delta_{1}$ e $\beta_{2}=1+\delta_{2}$, para $0<\delta_{1}, \delta_{2} \leqslant 2$. A matriz $\Delta$ toma neste caso a forma

$$
\Delta=\frac{\sigma^{2}}{n}\left(\frac{\nu+m+2}{\nu+m}\right) \mathrm{C}\left(\sum_{i=1}^{n} \mathbf{X}_{i}^{T} \mathbf{R}^{-1} \mathbf{X}_{i}\right)^{-1} \mathbf{C}^{T}
$$

em que $\mathrm{X}_{i}=\left[\mathbf{1}_{m}, x_{1 i} \mathbf{1}_{m}, x_{2 i} \mathbf{1}_{m}\right]$. A região menos favorável ocorre para ambas as estruturas em $\varrho=-\operatorname{corr}\left(x_{1}, x_{2}\right)$. Então, $\mathrm{P}=0,5 \operatorname{Pr}\left\{\chi_{1}^{2} \geqslant t\right\}+0,24 \operatorname{Pr}\left\{\chi_{2}^{2} \geqslant t\right\}$, $\mathrm{P}=0,5 \operatorname{Pr}\left\{\chi_{1}^{2} \geqslant t\right\}+0,27 \operatorname{Pr}\left\{\chi_{2}^{2} \geqslant t\right\}, t>0$ e $\mathrm{P}=0,5 \operatorname{Pr}\left\{\chi_{1}^{2} \geqslant t\right\}+0,25 \operatorname{Pr}\left\{\chi_{2}^{2} \geqslant\right.$ $t\}, t>0$, para $n=20, n=50$ e $n=100$, respectivamente.

Os resultados deste estudo são descritos nas Tabelas 6.9-6.11. Podemos notar, nessas tabelas, tendências semelhantes àquelas observadas para os dados agrupados (caso II). Em particular, podemos notar que o poder cresce, à medida que a correlação diminui. Esse resultado pode ser explicado pelo fato de que, quanto maior é a correlação menos informação tem-se da relação entre $\mu_{i}$ e $\left(x_{1 i}, x_{2 i}\right)$, e por conseguinte, dos valores de $\beta_{1}$ e $\beta_{2}$. As Figuras 6.9-6.12 exibem as distribuições nulas teórica e empírica da estatística $\xi_{R V}$. Podemos, também, notar aqui uma boa concordância entre as duas distribuições para $n=50$. Contudo, a distribuição empírica parece subestimar a distribuição nula para $n=20$. A Tabela 6.12 exibe as diferenças entre a distribuições nulas teórica e empírica da $\xi_{R V}$ para alguns percentis da distribuição nula assintótica. Similar ao caso II as diferenças parecem crescer quando a correlação cresce, porém, as diferenças máximas não são maiores do que $2 \%$ apenas para $n=50$. Esses resultados estão de acordo com os resultados apresentados por Fahrmeir e Klinger (1994) que comparam as distribuições nulas empírica e teórica de testes unilaterais em modelos lineares generalizados com respostas independentes, encontrando uma boa concordância para tamanhos amostrais pelo menos iguais a 50. Resultados similares foram também obtidos por Cardoso-Neto e Paula (2001), que aplicam esse tipo de procedimento em equações de estimação generalizadas. 


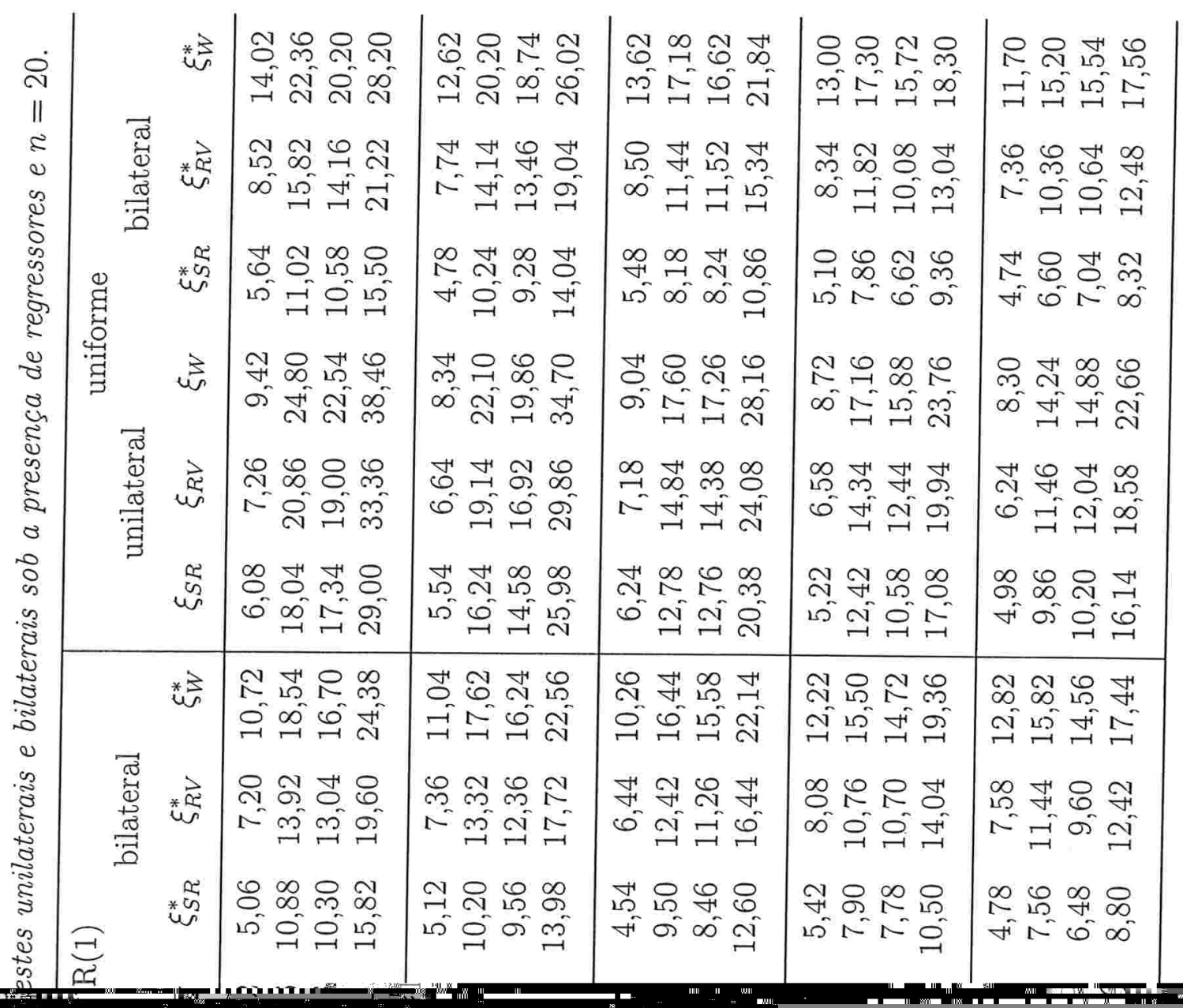




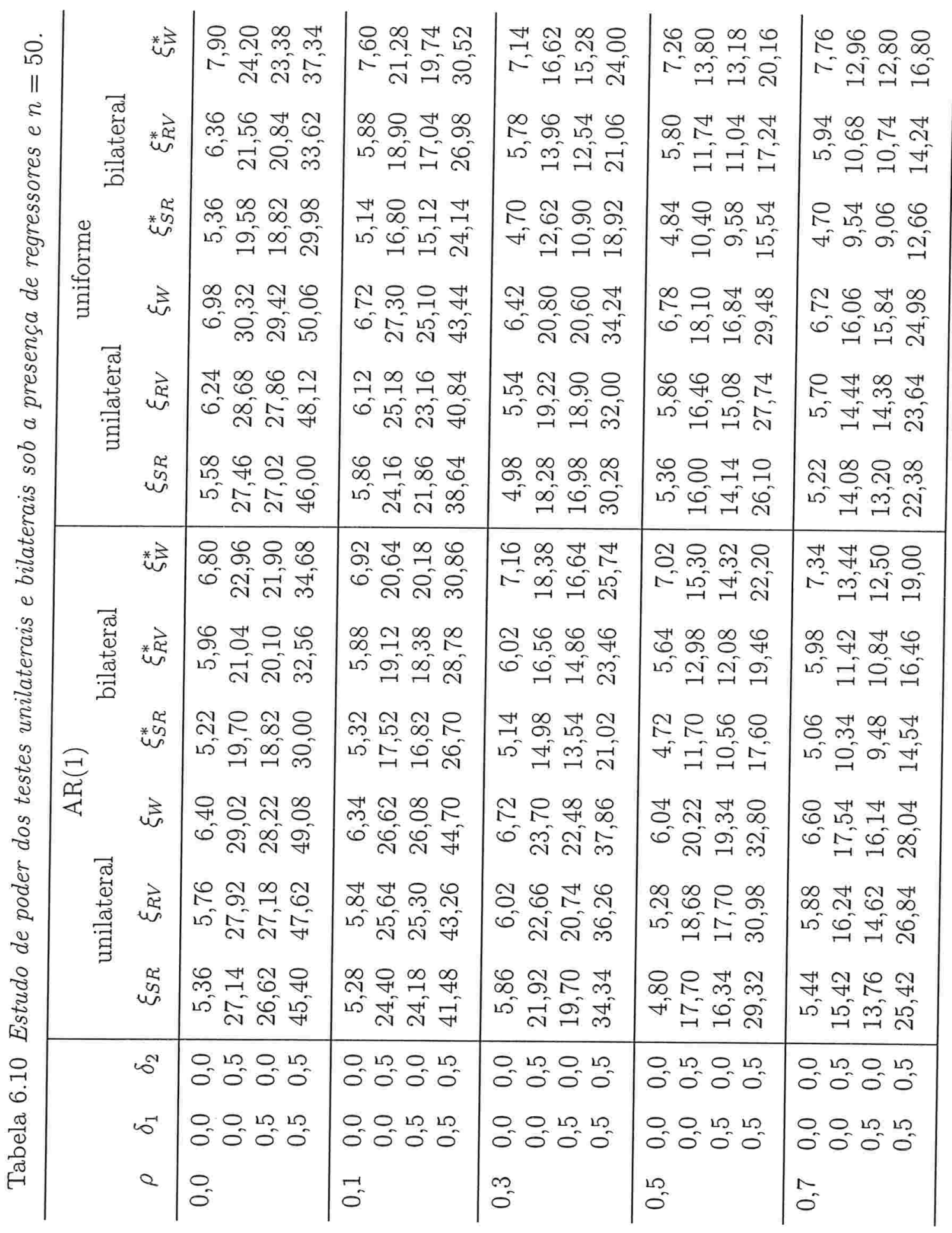




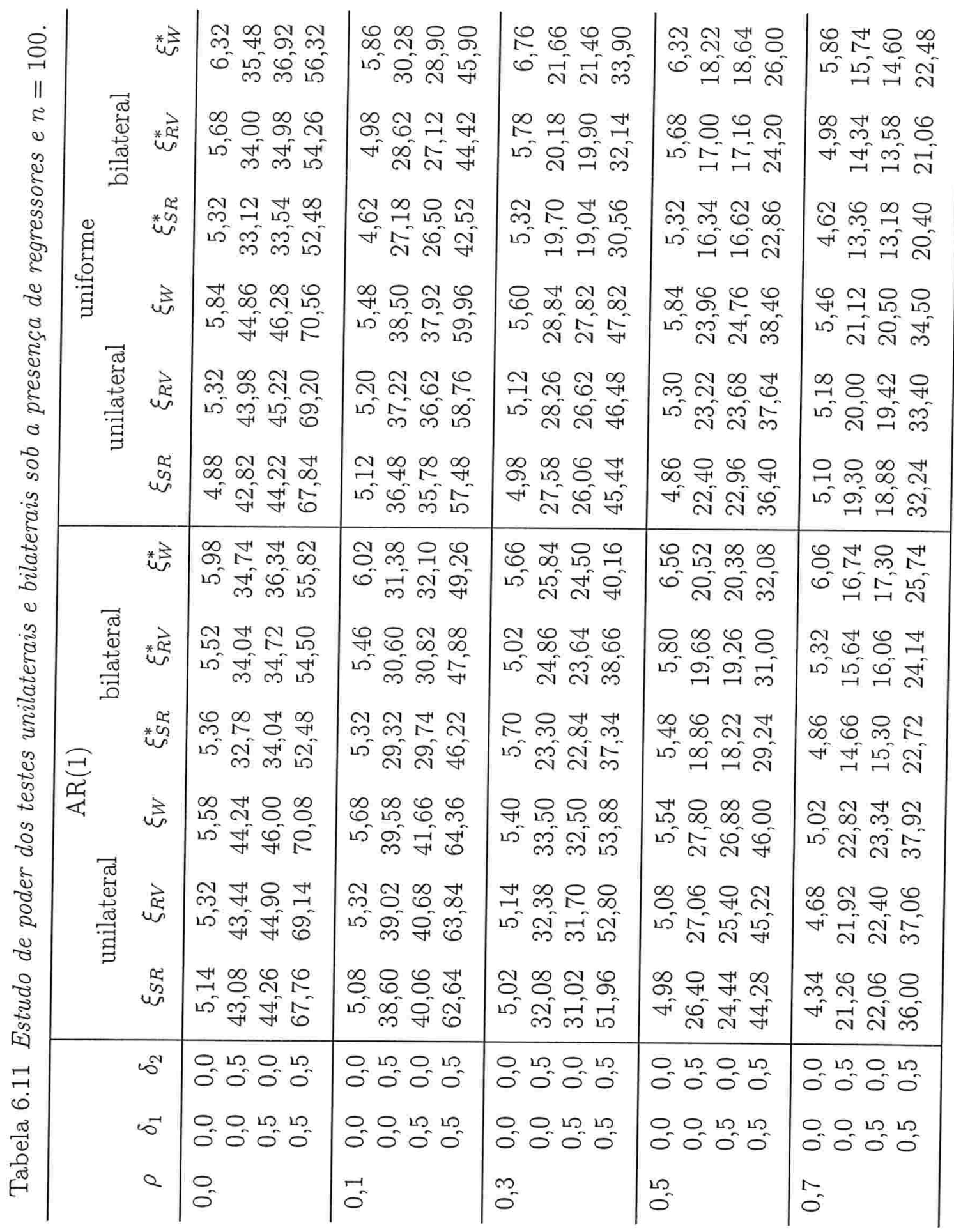


Figura 6.9 Distribuições acumuladas teórica e empírica da estatística $\xi_{R V}$ para regressores, $n=20$ e para a estrutura $\mathrm{AR}(1)$.
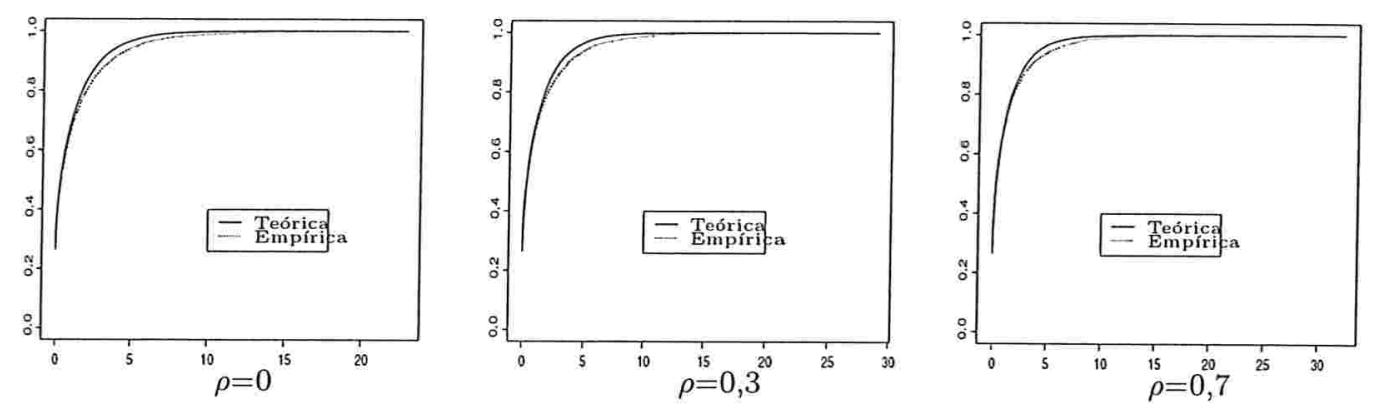

Figura 6.10 Distribuições acumuladas teórica e empírica da estatística $\xi_{R V}$ para regressores, $n=20$ e para a estrutura uniforme.
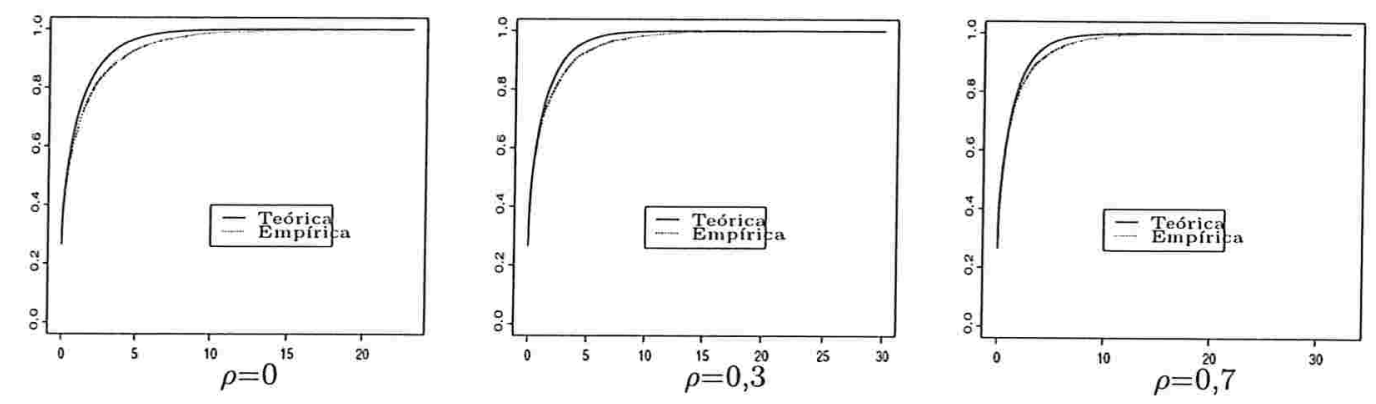

Figura 6.11 Distribuições acumuladas teórica e empírica da estatística $\xi_{R V}$ para regressores, $n=50$ e para a estrutura $\mathrm{AR}(1)$.
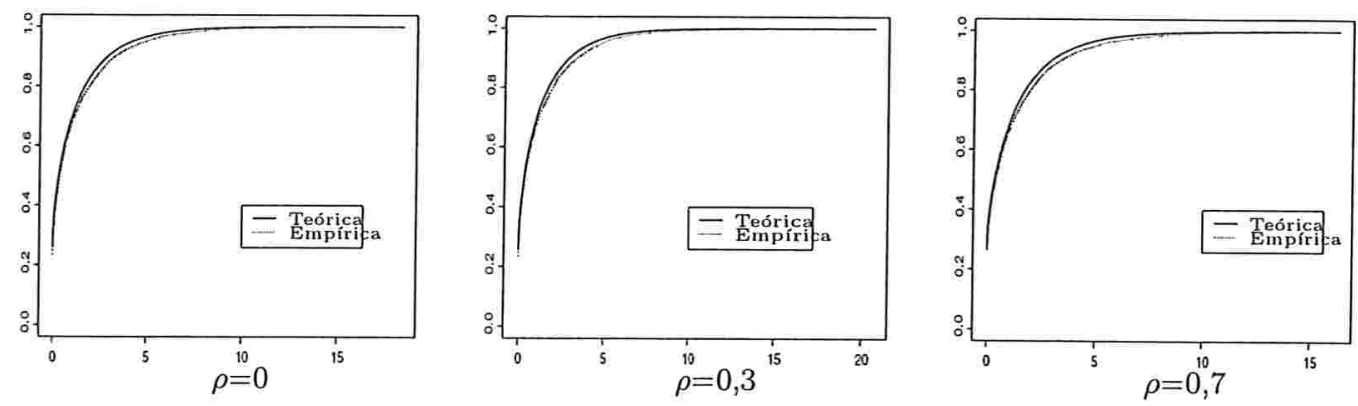
Figura 6.12 Distribuições acumuladas teórica e empírica da estatística $\xi_{R V}$ para regressores, $n=50$ e para a estrutura uniforme.
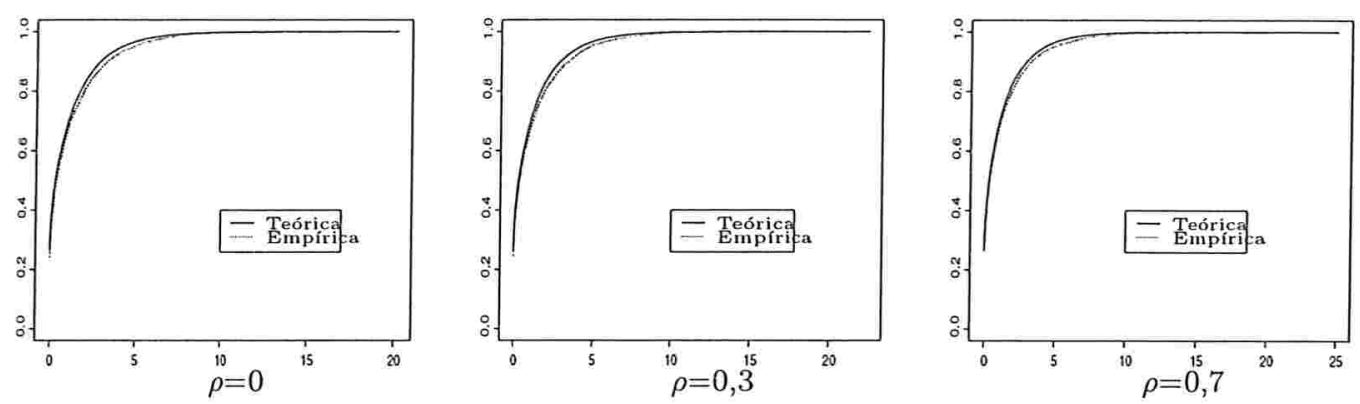

Tabela 6.12 Diferenças (em valor absoluto) entre as distribuições acumuladas teórica e empírica da estatística $\xi_{R V}$ para o caso de regressores (em \%).

\begin{tabular}{|c|c|c|c|c|c|c|c|c|c|c|c|c|c|}
\hline & \multirow[b]{2}{*}{$\rho$} & \multicolumn{6}{|c|}{$\begin{array}{c}\text { AR.(1) } \\
\text { percentil teórico }\end{array}$} & \multicolumn{6}{|c|}{$\begin{array}{c}\text { uniforme } \\
\text { percentil teórico }\end{array}$} \\
\hline & & $25 \%$ & $50 \%$ & $75 \%$ & $90 \%$ & $95 \%$ & $\max$ & $25 \%$ & $50 \%$ & $75 \%$ & $90 \%$ & $95 \%$ & $\max$ \\
\hline \multirow[t]{3}{*}{$n=20$} & 0,0 & 0,8 & 2,2 & 2,7 & 3,1 & 3,2 & 3,3 & 1,0 & 3,3 & 4,5 & 4,7 & 4,7 & 4,8 \\
\hline & 0,3 & 1,0 & 2,0 & 2,8 & 3,4 & 3,5 & 3,8 & 1,3 & 2,6 & 3,7 & 4,4 & 4,5 & 4,9 \\
\hline & 0,7 & 1,0 & 2,0 & 2,7 & 3,9 & 4,4 & 5,3 & 1,5 & 2,4 & 3,1 & 5,0 & 5,8 & 6,5 \\
\hline \multirow[t]{3}{*}{$n=50$} & 0,0 & 0,1 & 0,3 & 1,0 & 1,5 & 1,6 & 1,8 & 0,4 & 0,6 & 1,2 & 1,4 & 1,5 & 1,9 \\
\hline & 0,3 & 0,4 & 0,7 & 1,3 & 1,6 & 1,6 & 1,8 & 0,4 & 1,0 & 1,4 & 1,8 & 1,9 & 2,1 \\
\hline & 0,7 & 0,7 & 1,3 & 1,7 & 2,8 & 3,1 & 3,3 & 0,8 & 1,1 & 1,6 & 2,2 & 3,0 & 3,4 \\
\hline
\end{tabular}

\subsubsection{Dados sobre diabéticos}

Vamos reanalizar nesta seção o exemplo 2.1 discutido por Crowder e Hand (1990) em um estudo comparativo entre grupos de diabéticos. Originalmente foram considerados quatro grupos de pacientes. Porém, para ilustrar a metodologia descrita nas seções anteriores, consideraremos apenas os primeiros três grupos (veja, discussão em Shin, Park e Park, 1996): grupo controle $\left(n_{1}=8\right)$, grupo diabético sem complicações $\left(n_{2}=6\right)$ e grupo diabético com hipertensão $\left(n_{3}=7\right)$. Para cada paciente a resposta é uma tarefa física medida nos tempos 1, 2, 3, 4, 5, 6, 8 e 10 minutos. Foram excluídas os tempos de 12 e 15 minutos por apresentarem um 
grande número de observações perdidas. Para as observações perdidas, será assumido que o mecanismo gerador é completamente aleatório. O conjunto de dados é descrito no Apêndice F. Seja $y_{i \ell j}$ a resposta da tarefa física observada para o $i$-ésimo paciente do $\ell$-ésimo grupo no tempo $j$. Assumiremos o modelo

$$
\mathrm{y}_{i \ell}=\mu_{\ell}+\epsilon_{i \ell}
$$

em que $\mu_{\ell}=\mu_{\ell} 1_{m}, \mathrm{y}_{i \ell}=\left(y_{i \ell 1}, \ldots, y_{i \ell m}\right)^{T}$ e $\epsilon_{i \ell} \sim t_{m}\left(0, \Phi_{i \ell}, \nu\right)$ com $\Phi_{i \ell}=\Phi=$ $\sigma^{2} \mathbf{R}(\rho)$ e $m=8$. Como sugerido por Shin, Park e Park (1996) uma estrutura de correlação $\mathrm{AR}(1)$ é assumida para $\mathbf{R}(\rho)$. Além disso, é razoável assumir as restrições $\mu_{1} \geqslant \mu_{2} \geqslant \mu_{3}$ para os valores esperados da tarefa física.

As hipóteses de interesse aqui são $\mathrm{H}_{0}: \mu_{1}=\mu_{2}=\mu_{3}$ contra $\mathrm{H}_{2}: \mu_{1} \geqslant \mu_{2} \geqslant \mu_{3}$, com pelo menos uma desigualdade estrita em $\mathrm{H}_{2}$. A Tabela 6.13 apresenta as estimativas de máxima verossimilhança sob $\mathrm{H}_{0}$ e $\mathrm{H}_{2}$, bem como os valores das estatísticas dos testes da razão de verossimilhanças, Wald e escore ( $p$-valor entre parênteses) para o caso normal. As expressões das estatísticas são omitidas aqui, mas elas podem ser encontradas, por exemplo, em Gouriéroux, Holly e Monfort (1982). As distribuições nulas asintóticas das três estatísticas são equivalentes e segue uma mistura de distribuições qui-quadrados com pesos $\omega(0,2 ; \Delta)=0,344$, $\omega(1,2 ; \Delta)=0,5$ e $\omega(2,2 ; \Delta)=0,156$. Apresentamos também na Tabela 6.13 os resultados dos testes estatísticos para as hipóteses $\mathrm{H}_{0}: \mu_{1}=\mu_{2}=\mu_{3}$ contra $\mathrm{H}_{1}$ : pelo menos duas médias diferentes. Podemos notar pelos $p$-valores que a hipótese nula não é rejeitada ao nível de $10 \%$.

A Figura 6.13 apresenta o gráfico de índices do resíduo padronizado $\mathbf{t}_{r}^{*}=\left(\mathbf{t}_{r_{11}}^{T}, \ldots\right.$, $\left.\mathbf{t}_{r_{n_{1} 1}}^{T}, \mathbf{t}_{r_{12}}^{T}, \ldots, \mathbf{t}_{r_{n_{2} 2}}^{T}, \mathbf{t}_{r_{13}}^{T}, \ldots, \mathbf{t}_{r_{n_{3} 3}}^{T}\right)^{T}$ em que $\mathbf{t}_{r_{i \ell}}=\hat{\Phi}^{-1 / 2}\left(\mathbf{y}_{i \ell}-\hat{\mu}_{\ell}\right)$. O valor mais extremo foi observado para o paciente 6 no grupo 3 . A eliminação desse paciente leva à rejeição da hipótese nula ao nível de 5\%. O gráfico normal de probabilidades para $t_{r_{k}}^{*}$ com envelope gerado como sugerido por Atkinson (1981) é dado na Figura 6.14. O gráfico indica que uma distribuição com caudas mais pesadas para o erro pode ser mais apropriada.

A Tabela 6.14 apresenta os resultados segundo modelo $t$-Student com $\nu=15$ 
Tabela 6.13 Estimativas de máxima verossimilhança e valores das estatísticas dos testes sob o modelo normal.

\begin{tabular}{rrrrrrr}
\hline Parâmetro & \multicolumn{2}{c}{$\mathrm{H}_{0}$} & \multicolumn{2}{c}{$\mathrm{H}_{2}$} & \multicolumn{2}{c}{$\mathrm{H}_{1}$} \\
\hline$\mu_{1}$ & 6,658 & $(0,792)$ & 7,209 & $(1,251)$ & 6,624 & $(1,237)$ \\
$\mu_{2}$ & 6,658 & $(0,792)$ & 7,209 & $(1,445)$ & 7,989 & $(1,428)$ \\
$\mu_{3}$ & 6,658 & $(0,792)$ & 5,554 & $(1,337)$ & 5,553 & $(1,322)$ \\
$\sigma^{2}$ & 15,057 & $(4,094)$ & 14,410 & $(3,898)$ & 14,119 & $(3,810)$ \\
$\rho$ & 0,960 & $(0,012)$ & 0,958 & $(0,012)$ & 0,957 & $(0,012)$ \\
$\xi_{S R}$ & $\xi_{R V}$ & $\xi_{W}$ & $\xi_{S R}^{*}$ & $\xi_{R V}^{*}$ & $\xi_{W}^{*}$ & \\
0,971 & 0,995 & 1,021 & 1,459 & 1,512 & 1,569 & \\
$(0,258)$ & $(0,254)$ & $(0,250)$ & $(0,482)$ & $(0,470)$ & $(0,457)$ & \\
\hline
\end{tabular}

Figura 6.13 Gráfico de índices do resíduo padronizado $t_{r_{k}}^{*}$ sob o modelo normal.

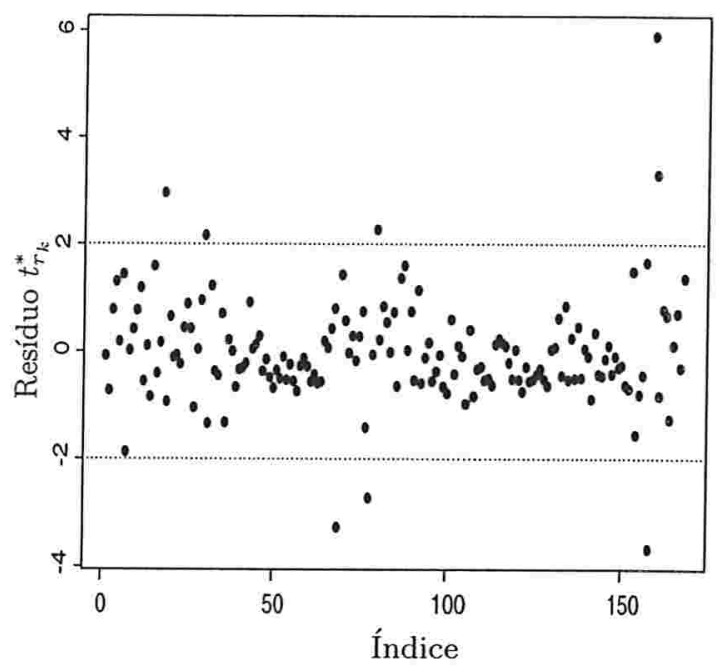

graus de liberdade para os erros. $\mathrm{O}$ valor $\nu=15$ foi obtido pela maximização do logaritmo da função de verossimilhança $L\left(\boldsymbol{\beta}, \sigma^{2}, \rho, \nu\right)$. Fernandez e Steel (1999) mostram que quando $\nu$ é desconhecido a função $\mathrm{L}\left(\boldsymbol{\beta}, \sigma^{2}, \rho, \nu\right)$ é não limitada, à me- 
Figura 6.14 Gráfico normal de probabilidades com envelope para o resíduo padronizado $t_{r_{k}}^{*}$ sob o modelo normal.

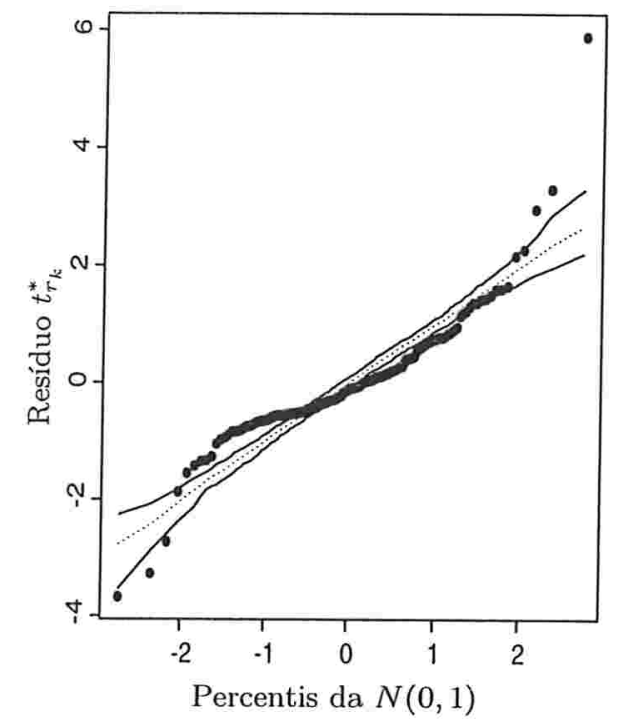

dida que o espaço paramétrico tende para a fronteira. Uma consequência desse fato é que as estimativas de máxima verossimilhança podem corresponder ao máximo local e não ao global. Contudo, o máximo local pode nos fornecer informações sobre a região do máximo global. Verificamos que $\nu=15$ corresponde aproximadamente ao máximo global do logaritmo da função de verossimilhança para o conjunto de dados do exemplo. Pela Tabela 6.14 nota-se que as estimativas de máxima verossimilhança sob o modelo $t$-Student são, em geral, menores do que as estimativas sob o modelo normal. Os pesos que aparecem na distribuição nula assintótica dos testes unilaterais assumem os mesmos valores dos pesos do caso normal. Contudo, os $p$-valores dos testes estatísticos indicam pela rejeição da hipótese nula ao nível de 10\%. Assim, sob modelo $t$-Student $\operatorname{com} \nu=15$ graus de liberdade existe indicação de que, pelo menos, o grupo de diabéticos com hipertensão apresenta valor esperado para a tarefa física menor do que os valores esperados para o grupo controle. 
Tabela 6.14 Estimativas de máxima verossimilhança e valores das estatísticas dos testes sob o modelo $t$-Student com $\nu=15$ graus de liberdade.

\begin{tabular}{rrrrrrr}
\hline Parâmetro & \multicolumn{2}{c}{$\mathrm{H}_{0}$} & \multicolumn{2}{c}{$\mathrm{H}_{2}$} & \multicolumn{2}{c}{$\mathrm{H}_{1}$} \\
\hline$\mu_{1}$ & 5,532 & $(0,738)$ & 6,439 & $(1,122)$ & 5,696 & $(1,100)$ \\
$\mu_{2}$ & 5,532 & $(0,738)$ & 6,439 & $(1,295)$ & 7,513 & $(1,270)$ \\
$\mu_{3}$ & 5,532 & $(0,738)$ & 3,995 & $(1,199)$ & 3,994 & $(1,176)$ \\
$\sigma^{2}$ & 11,482 & $(3,529)$ & 10,216 & $(3,111)$ & 9,845 & $(2,991)$ \\
$\rho$ & 0,974 & $(0,008)$ & 0,971 & $(0,009)$ & 0,970 & $(0,009)$ \\
$\xi_{S R}$ & $\xi_{R V}$ & $\xi_{W}$ & $\xi_{S R}^{*}$ & $\xi_{R V}^{*}$ & $\xi_{W}^{*}$ & \\
2,724 & 2,730 & 2,768 & 3,503 & 3,781 & 4,138 & \\
$(0,090)$ & $(0,089)$ & $(0,087)$ & $(0,174)$ & $(0,151)$ & $(0,126)$ & \\
\hline
\end{tabular}

A Figura 6.15 apresenta o gráfico de índices $t_{r_{k}}^{*}$ em que $\mathbf{t}_{r}^{*}=\left(\mathbf{t}_{r_{11}}^{T}, \ldots, \mathbf{t}_{r_{n_{1} 1}}^{T}, \mathbf{t}_{r_{12}}^{T}\right.$, $\left.\ldots, \mathbf{t}_{r_{n_{2}}}^{T}, \mathbf{t}_{r_{13}}^{T}, \ldots, \mathbf{t}_{r_{n_{3} 3}}^{T}\right)^{T}$ com $\mathbf{t}_{r_{i \ell}}=\left[\frac{\nu}{\nu-2} \hat{\Phi}\right]^{-1 / 2}\left(\mathbf{y}_{i \ell}-\hat{\mu}_{\ell}\right)$, indicando o mesmo valor extremo que aparece na Figura 6.13. A eliminação do paciente 6 no grupo 3 não muda a decisão de rejeição da hipótese nula a 10\%, mostrando a robustez do teste unilateral sob o modelo $t$-Student neste exemplo contra observações extremas. O gráfico de probabilidades $t_{r_{k}}^{*}$ (Figura 6.16) indica alguma evidência de que o modelo $t$-Student com $\nu=15$ graus de liberdade parece ser menos inapropriado para ajustar os dados do que o modelo normal. O comportamento do $p$-valor contra os graus de liberdade para as três estatísticas (Figura 6.17) indica que a especificação incorreta de $\nu$, por exemplo, assumindo um número menor de graus de liberdade para acomodar os pontos aberrantes, pode dificultar na detecção de diferenças acerca das médias. Finalmente, na Figura 6.18 temos as distribuições nulas empírica e teórica de $\xi_{R V}$ sob o modelo $t$-Student ajustado, indicando que a distribuição empírica tende a subestimar a distribuição teórica. Portanto, os $p$-valores do testes unilaterais sob o modelo $t$-Student podem estar sendo subestimados neste exemplo. 
Figura 6.15 Gráfico de índices do resíduo padronizado $t_{r_{k}}^{*}$ sob o modelo $t$-Student.

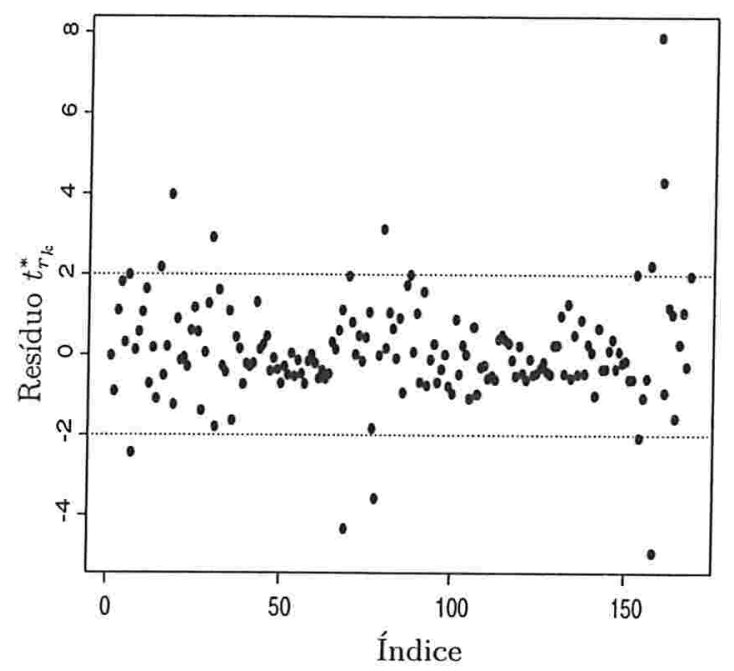

Figura 6.16 Gráfico de probabilidades com envelope para o resíduo padronizado $t_{r_{k}}^{*}$ sob o modelo t-Student.

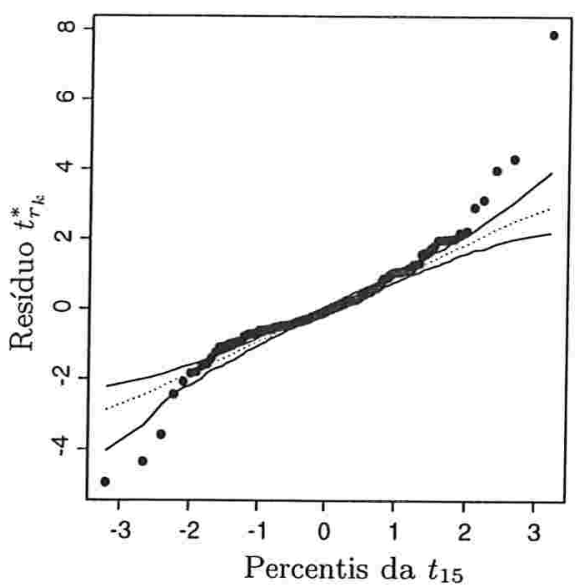


Figura 6.17 Comportamentos do p-valor para as estatísticas $\xi_{S R}$, $\xi_{R V}$ e $\xi_{W}$ sobo modelo t-Student ajustado aos dados de diabéticos.

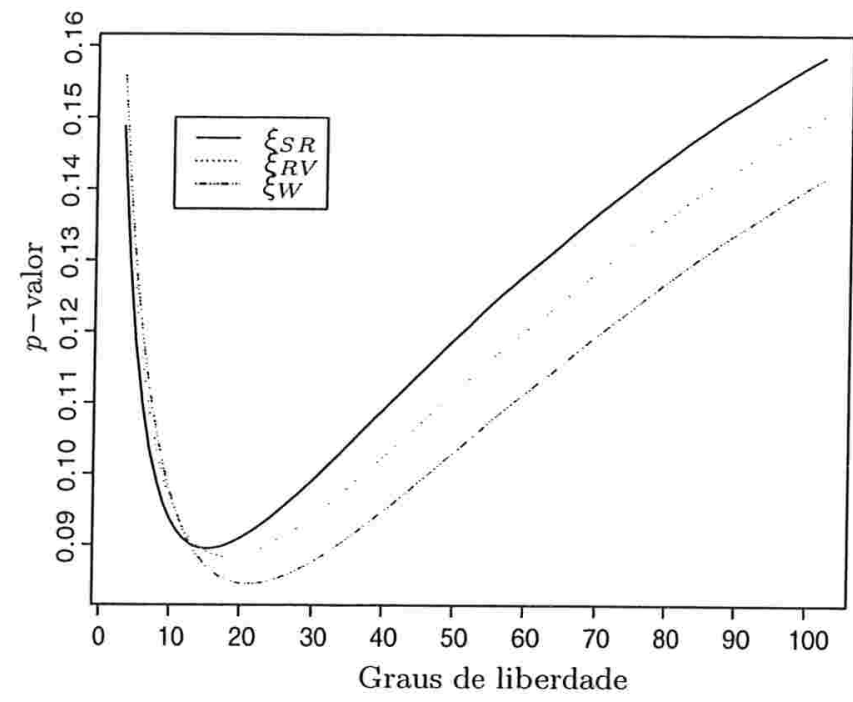

Figura 6.18 Distribuições acumuladas teórica e empírica da estatística $\xi_{R V}$ sob o modelo t-Student ajustado aos dados de diabéticos.

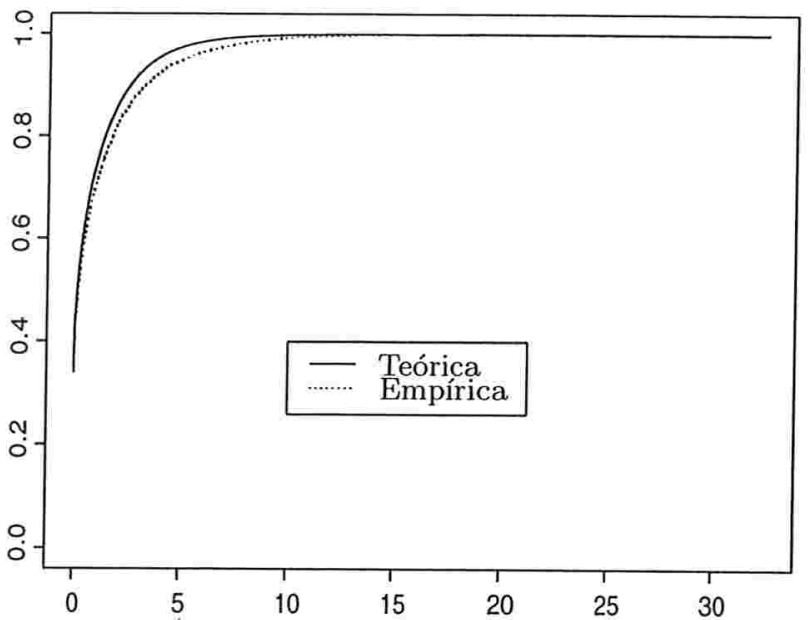




\section{Conclusões}

Em resumo, nesta tese discutimos vários aspectos envolvendo modelos simétricos de regressão. Um dos aspectos abordados foi o desenvolvimento de métodos de diagnóstico em modelos simétricos lineares e não-lineares. Em particular, desenvolvemos métodos para detectar pontos de alavanca e mostramos que a matriz generalizada de pontos de alavanca pode ser decomposta em duas partes, uma devido aos parâmetros de locação e outra devido aos parâmetros de escala. Verificamos, também, a conexão entre essas medidas de influência local quando perturbações na resposta são feitas. Temos notado que, para algumas aplicações, os modelos simétricos em que a distribuição dos erros tem caudas mais pesadas do que a normal, tendem a acomodar melhor as observações aberrantes. Quando a suposição de homoscedasticidade dos erros não é verificada, modelos simétricos lineares heteroscedásticos foram propostos. Desenvolvemos um processo iterativo para a estimação dos parâmetros de locação e escala, bem como, métodos de validação baseado em influência local. Propomos um resíduo padronizado para os modelos simétricos lineares e não-lineares. Estudos de simulação mostraram que este resíduo tem média zero e variância um, uma assimetria desprezível e uma curtose acompanhando a curtose da distribuição.

Outro aspecto abordado foi na área de modelos restritos, em que desenvolvemos processos iterativos para a estimação de parâmetros restritos em igualdades e desigualdades lineares, relativamente simples de serem implementados nos modelos simétricos lineares. Sob certas condições de regularidade verificamos que a distribuição nula assintótica das estatísticas dos testes unilaterais é uma mistura de qui-quadrados, unicamente determinada em ambos os casos estudados. As estimativas de máxima verossimilhança para os coeficientes de regressão como esperado 
são, em geral, robustas a observações aberrantes e ou influentes para alguns modelos simétricos. Essas propriedades foram confirmadas no exemplo, bem como no estudo de sensitividade apresentado no Capítulo 5.

As propriedades descritas acima de robustez foram, também, observadas nos modelos $t$-multivariados. Verificamos neste caso que a distribuição nula assintótica é única, isto é, não depende de parâmetros sob a hipótese nula, porém pode depender das correlações. Estudos de simulação indicam uma boa concordância entre as distribuições nulas teórica e empírica de alguns testes unilaterais mesmo para amostras pequenas $(n=20)$. Foi confirmado nesses estudos a superioridade dos testes unilaterais sobre os testes bilaterais, em particular perto dos valores dos parâmetros sob a hipótese nula.

Várias linhas de pesquisa podem ser ainda tratadas, tais como :

(i) definir outros tipos de resíduos, tal como o resíduo componente do desvio para os modelos simétricos;

(ii) estender os modelos não-lineares, definir resíduos e medidas de diagnóstico para outras classes de distribuições, por exemplo, skew-elíptica;

(iii) estender os resultados de diagnósticos para modelos mistos simétricos;

(iv) encontrar casos particulares em que os pesos não dependam dos parâmetros;

$(v)$ estender os resultados encontrados para o modelo t-multivariado para a classe elíptica multivariada;

(vi) definir medidas de diagnóstico para os modelos simétricos restritos;

(vii) discutir métodos restritos em modelos simétricos mistos. Por exemplo, testes para avaliar a presença de efeito aleatório;

(viii) estender os métodos restritos para a classe dos modelos simétricos heteroscedásticos.

Concluindo, esta tese é um esforço inicial para apresentar alguns tópicos nesta área de pesquisa e divulgar a utilidade da mesma. 


\section{APENDICE A}

\section{Medidas de curvatura e viés de ordem $n^{-1}$}

Nesse apêndice o objetivo principal é desenvolver medidas de não-linearidade em modelos simétricos não-lineares. Essas medidas podem indicar se o grau de nãolinearidade de um problema de estimação é suficientemente pequeno para que os procedimentos usuais de estimação desenvolvidos, no caso linear, possam ser utilizados como uma boa aproximação para o caso não-linear. Os primeiros esforços no desenvolvimento de uma medida de não-linearidade foi devido a Beale (1960). Uma importante contribuição foi dada por Box (1971) que obteve a aproximação de ordem $n^{-1}$ para o viés do estimador de máxima verossimilhança de $\hat{\beta}$ sob erro normal. Cordeiro, Ferrari, Uribe-Opazo e Vasconcellos (2000) estenderam esses resultados para os modelos simétricos. Existem, contudo, diversos outros trabalhos envolvendo o cálculo do viés de ordem $n^{-1}$ em modelos de regressão. Por exemplo, Cordeiro e McCullagh (1991) e Paula (1992) obtiveram tais expressões para os estimadores de máxima verossimilhança em modelos lineares generalizados e modelos não-lineares de família exponencial, respectivamente. Um dos trabalhos mais relevantes no assunto é devido a Bates e Watts (1980) que utilizam alguns conceitos de geometria diferencial para desenvolver medidas de curvatura em modelos normais não-lineares. Estendemos essas medidas para os modelos simétricos não-lineares.

\section{A.1 Multiplicação de "array"}

A multiplicação de "arrays" tri-dimensionais foi primeiro introduzida por Bates e Watts (1980). Desde então, muitos autores têm discutido e utilizado essa multiplicação (veja Bates e Watts, 1988; Seber e Wild, 1989, pp.691-692 e Wei, 1998, pp.188-190).

Um "array" tri-dimensional de dimensão $n \times p \times q$ é denotado por $\mathrm{X}=\left(X_{\ell i j}\right)$, 
em que os índices $\ell, i$ e $j$ indicam a face, a linha e a coluna, respectivamente. Um "array" pode ser visto da seguinte forma: $\mathbf{A}=\left(\mathrm{A}_{\ell}\right)$ e cada $\mathbf{A}_{\ell}$ é uma matriz $\mathrm{A}_{\ell}=\left(A_{\ell i j}\right)$ de dimensão $p \times q$ para algum $\ell$ fixo e $\mathbf{A}_{\ell}$ é chamada de $\ell$-ésima face de A.

Definição A.1 Se X é um "array" de dimensão $n \times p \times q$, A e B são matrizes de dimensões $r \times p$ e $q \times s$, respectivamente, então $Y=A X B$ é definido como um "array" de dimensão $n \times r \times s$ com elementos:

$$
Y_{\ell k t}=\sum_{i=1}^{p} \sum_{j=1}^{q} A_{k i} X_{\ell i j} B_{j t} .
$$

Definição A.2 Se X é um "array" de dimensão $n \times p \times q, A$ é uma matriz de dimensão $m \times n$, então $Y=[A][X]$ é chamado de produto colchete de $A$ e $X$, isto é, um "array" de dimensão $m \times p \times q$ com elementos:

$$
Y_{\ell i j}=\sum_{t=1}^{n} A_{\ell k} X_{t i j} .
$$

A seguinte propriedade pode ser deduzida diretamente das definições acima :

Propriedade A.1 Sejam A,L, M matrizes e X um "array", então, temos que

$$
[A][L X M]=L[A][X] M .
$$

\section{A.2 Medidas de curvatura}

Considere, então, o modelo de regressão simétrico definido na Seção 2.2. Uma linha arbitrária no espaço paramétrico que passa através de $\hat{\beta}$ pode ser expressa usando um parâmetro $b$ dado por

$$
\boldsymbol{\beta}(b)=\hat{\boldsymbol{\beta}}+b \mathbf{h},
$$

em que $\mathbf{h}=\left(h_{1}, \ldots, h_{p}\right)^{T}$ é algum vetor não nulo. Essa linha gera uma curva, ou "linha projetada" sobre o espaço solução, definida por

$$
\eta_{h}(b)=\eta(\hat{\beta}+b \mathbf{h})
$$


em que $\eta_{h}(b)$ é o ponto móvel no "tempo" $b$. A curva tangente no ponto $b=0$ é expressa na forma

$$
\dot{\eta}_{h}(b)=\mathrm{D}_{\hat{\beta}} \mathrm{h},
$$

em que $\mathbf{D}_{\hat{\beta}}$ é aqui a matriz Jacobiana da transformação $\eta(\boldsymbol{\beta})$ em $\boldsymbol{\beta}=\hat{\boldsymbol{\beta}}$. O conjunto de todas as combinações lineares de (A.1) é também chamado de plano tangente em $\eta(\hat{\boldsymbol{\beta}})$.

A aceleração da curva $\eta_{h}$ ou vetor de aceleração é definido por

$$
\ddot{\eta}_{h}=\mathrm{h}^{T} \mathrm{D}_{\hat{\beta} \hat{\beta}} \mathbf{h}
$$

em que $\hat{\mathbf{D}}_{\beta \beta}$ é um "array" de dimensão $n \times p \times p$ com $i$-ésima face dada por $\mathrm{D}_{\beta \beta}(i)=\frac{\partial^{2} \eta_{i}}{\partial \beta_{r} \partial \beta_{s}}, i=1, \ldots, n$ e $r, s=1, \ldots, p$.

Portanto, cada elemento do "array" $\ddot{\eta}_{h}$ é dado por $\mathbf{h}^{T} \mathbf{D}_{\hat{\beta} \hat{\beta}}(i) \mathbf{h}, i=1, \ldots, n$. O vetor de aceleração $\ddot{\eta}_{h}$ pode ser decomposto em três componentes. A primeira componente $\ddot{\eta}^{I N}$ determina a variação na direção do vetor de velocidade instantânea $\dot{\eta}_{h}$ normal ao plano tangente, enquanto a segunda componente $\ddot{\eta}^{G}$ determina a variação na direção $\ddot{\eta}_{h}$ paralela ao plano tangente e a terceira componente $\ddot{\eta}^{P}$ a variação na velocidade do ponto móvel em que $\ddot{\eta}^{P E}=\ddot{\eta}^{G}+\ddot{\eta}^{P}$. Essas componentes foram transformadas por Bates e Watts (1980) nas curvaturas intrínseca e paramétrica definidas por $K_{h}^{I N}=\frac{\ddot{\eta}^{I N}}{\left\|\dot{\eta}_{h}\right\|^{2}}$ e $K_{h}^{P E}=\frac{\ddot{\eta}^{P E}}{\left\|\dot{\eta}_{h}\right\|^{2}}$, respectivamente.

Essas curvaturas podem ser padronizadas tal que sejam invariantes com mudanças de escala. Para isto, suponha $\boldsymbol{\beta}$ próximo de $\hat{\boldsymbol{\beta}}$ de modo que possamos usar uma aproximação quadrática em Taylor

$$
\begin{aligned}
\mu-\hat{\mu} & =f(\boldsymbol{\beta})-f(\hat{\boldsymbol{\beta}}) \approx \mathrm{D}_{\hat{\beta}}(\boldsymbol{\beta}-\hat{\boldsymbol{\beta}})+\frac{1}{2}(\boldsymbol{\beta}-\hat{\boldsymbol{\beta}})^{T} \mathrm{D}_{\hat{\beta} \hat{\beta}}(\boldsymbol{\beta}-\hat{\boldsymbol{\beta}}) \\
& =\mathrm{D}_{\hat{\beta}}(\boldsymbol{\beta}-\hat{\boldsymbol{\beta}})+\frac{1}{2} \delta^{T} \mathrm{D}_{\hat{\beta} \hat{\beta}} \delta
\end{aligned}
$$

em que $\boldsymbol{\delta}=\boldsymbol{\beta}-\hat{\boldsymbol{\beta}}$.

Ignorando o termo quadrático em (A.2), obtemos uma aproximação linear para $\boldsymbol{\beta}$ na vizinhança de $\hat{\beta}$

$$
\mu-\hat{\mu} \approx \mathrm{D}_{\hat{\beta}}(\beta-\hat{\beta}) .
$$


Pela equação (A.1) vimos que a faixa (espaço coluna) da matriz $D_{\hat{\beta}}$ é o plano tangente da superfície esperada no ponto $\hat{\beta}$ e a equação (A.3) mostra que a aproximação $\boldsymbol{\mu}$ pertence a esse plano tangente. Portanto, a aproximação linear (A.3) é igual à aproximação da superfície esperada na vizinhança de $\hat{\boldsymbol{\beta}}$ pelo plano tangente em $\hat{\boldsymbol{\beta}}$. Então, podemos construir uma região de $100(1-\alpha) \%$ de confiança para $\boldsymbol{\beta}$ que é dada pelo conjunto de valores de $\beta$ no plano tangente, tais que

$$
\|\boldsymbol{\mu}-\hat{\boldsymbol{\mu}}\|^{2} \approx\left\|\mathrm{D}_{\hat{\boldsymbol{\beta}}}(\boldsymbol{\beta}-\hat{\boldsymbol{\beta}})\right\|^{2} \leqslant \rho^{2} \chi_{\alpha}^{2}
$$

em que $\chi_{\alpha}^{2}=\chi_{p, \alpha}^{2}$ é o percentil de ordem $(1-\alpha)$ da distribuição $\chi^{2}$ com $p$ graus de liberdade e $\rho=\sqrt{\phi\left(4 d_{g}\right)^{-1}}$. A equação (A.4) mostra que $\mu$ pertence aproximadamente à esfera de raio $\rho \sqrt{\chi_{\alpha}^{2}}$ e centro $\mu$. Reescrevendo (A.4), temos que $(\boldsymbol{\beta}-\hat{\boldsymbol{\beta}})^{T} \mathbf{D}_{\hat{\boldsymbol{\beta}}}^{T} \mathbf{D}_{\hat{\boldsymbol{\beta}}}(\boldsymbol{\beta}-\hat{\boldsymbol{\beta}}) \leqslant \rho^{2} \chi_{\alpha}^{2}$ é um elipsóide de centro $\hat{\boldsymbol{\beta}}$. Sendo assim, podemos usar $\rho$ como um fator de escala, e similarmente a Bates e Watts (1980) se dividirmos $\mathrm{y}, \boldsymbol{\mu}, \hat{\mu}, \mathrm{D}_{\hat{\beta}}$ e $\mathrm{D}_{\hat{\beta} \hat{\beta}}$ por $\rho$ nas duas curvaturas acima e na curvatura da esfera (inverso do raio) em (A.4), obtemos

$$
\gamma_{h}^{I N}:=\rho K_{h}^{I N}, \gamma_{h}^{P E}:=\rho K_{h}^{P E} \mathrm{e} \frac{1}{\sqrt{\chi_{\alpha}^{2}}},
$$

respectivamente. Essa normalização será adotada nesta seção. Denotando por $\mathrm{V}$. = $\mathbf{D}_{\beta} / \rho$ e $\mathbf{V}_{. .}=\mathbf{D}_{\beta \beta} / \rho$ e seja a decomposição QR (Businger e Golub, 1965) da matriz $\mathrm{V}$. = $\mathrm{QR}$ em que $\mathrm{Q}$ é uma matriz ortogonal $n \times n$ e $\mathbf{R}$ é uma matrix $n \times p$ definida por

$$
\mathbf{R}=\left[\begin{array}{c}
\tilde{R} \\
0
\end{array}\right]
$$

com $\tilde{\mathbf{R}}$ sendo uma matriz triangular superior $p \times p$ e inversível. Seja o "array", $\mathbf{U}=\mathbf{L}^{T} \hat{\mathbf{V}}$..L em que $\mathbf{L}=\tilde{\mathbf{R}}^{-1}$. Denote os elementos de $\mathbf{U}$, "arrays" $n \times 1$ por $\mathbf{U}_{k j}, k, j=1, \ldots, p$ e definindo o vetor de aceleração $n \times p \times p, \mathbf{A}=\mathbf{Q}^{T} \mathbf{U} . \mathrm{O}$ $(k, j)$-ésimo elemento deste vetor de dimensão $n \times 1$ fica expresso na forma $\mathrm{Q}^{T} \mathrm{U}_{k j}$. Então, o "array" A é dado por

$$
\mathrm{A}=\left[\begin{array}{ccc}
\mathrm{Q}^{T} \mathrm{U}_{11} & \ldots & \mathrm{Q}^{T} \mathrm{U}_{1 p} \\
\vdots & \vdots & \vdots \\
\mathrm{Q}^{T} \mathrm{U}_{p 1} & \ldots & \mathrm{Q}^{T} \mathrm{U}_{p p}
\end{array}\right]
$$


VIÉS DE SEGUNDA ORDEM DAS ESTIMATIVAS DE MÁXIMA VEROSSIMILHANÇA

em que $\mathbf{Q}^{T} \mathbf{U}_{k j}=\left(a_{k j 1}, \ldots, a_{k j n}\right)^{T}$. A $i$-ésima face de A é expressa na forma

$$
\mathbf{A}_{i}=\left[\begin{array}{ccc}
a_{i 11} & \ldots & a_{i 1 p} \\
\vdots & \vdots & \vdots \\
a_{i p 1} & \ldots & a_{i p p}
\end{array}\right]
$$

para $i=1, \ldots, n$. Seja $\mathrm{A}^{I N}$ o "array" composto pelas $p$-primeiras faces de A e $\mathrm{A}^{P E}$, o "array" é composto pelas últimas $(n-p)$ faces de $\mathbf{A}$. Então, as medidas de não-linearidade serão dadas por $\gamma^{I N}=\max _{h}\left\|\mathbf{h}^{T} \mathbf{A}^{I N} \mathbf{h}\right\|$ e $\gamma^{P E}=$ $\max _{h}\left\|\mathbf{h}^{T} \mathbf{A}^{P E} \mathbf{h}\right\|$, em que $\|\mathbf{h}\|=1$. Desta forma, podemos usar o algorimo descrito por Bates e Watts (1980) que descreve uma metodologia para encontrar a curvatura máxima $\gamma_{\max }^{I N}$ e $\gamma_{\max }^{P E}$ para a respectiva maximização de $\gamma^{I N}$ e $\gamma^{P E}$. Similarmente, podemos sugerir um critério $\gamma^{I N}<2 \sqrt{\chi_{\alpha}^{2}}$ e $\gamma^{P E}<2 \sqrt{\chi_{\alpha}^{2}}$ indicando que a aproximação ao plano tangente é aceitável. Outro critério é baseado em $1-\left\{1-\left(\gamma_{h}^{I N}\right)^{2} \chi_{\alpha}^{2}\right\}^{1 / 2}$, em que essa quantidade será grande se $\gamma_{h}^{I N}$ for grande. Concluindo, a aproximação para o plano tangente será inaceitável se a curvatura máxima intrínseca for muito grande em $\hat{\beta}$.

\section{A.3 Viés de segunda ordem das estimativas de máxima verossimilhança}

Cordeiro, Ferrari, Uribe-Opazo e Vasconcellos (2000) expressam o viés de segunda ordem da estimativa de máxima verossimilhança de $\beta$, que pode ser reescrito como

$$
\mathrm{B}(\hat{\beta})=-\frac{\phi}{8 d_{g}}\left(\mathbf{D}_{\beta}^{T} \mathbf{D}_{\beta}\right)^{-1} \mathbf{D}_{\beta}^{T} \mathbf{z}
$$

em que $\mathbf{z}$ é um vetor $n \times 1$ com o $i$-ésimo elemento dado por $z_{i}=\operatorname{tr}\left\{\left(\mathbf{D}_{\beta}^{T} \mathbf{D}_{\beta}\right)^{-1} \mathbf{D}_{\beta \beta}(i)\right\}$ $i=1, \ldots, n$.

Portanto, o viés, $\mathrm{B}(\hat{\boldsymbol{\beta}})$ pode ser interpretado como a estimativa de mínimos quadrados da regressão de $\mathrm{z}$ nas colunas de $\mathbf{D}_{\beta}$ multiplicada por um fator de escala que depende da distribuição simétrica $\left(d_{g}\right)$ e do parâmetro de dispersão $(\phi)$. Sendo assim, o viés pode ser grande quando $d_{g}$ e $n$ são ambos pequenos. Além disso, o viés cresce com o parâmetro de dispersão. Nos modelos lineares, $\mathrm{B}(\hat{\beta})=0$ 
VIÉS DE SEGUNDA ORDEM DAS ESTIMATIVAS DE MÁXIMA VEROSSIMILHANÇA

pois $\mathrm{D}_{\beta \beta}(i)=0$ para todo $i$. Bates e Watts (1980) mostram que o viés de Box está relacionado com a medida de não-linearidade $\gamma^{P E}$ nos modelos normais nãolineares. Similarmente, temos que a relação entre $\mathrm{B}(\hat{\boldsymbol{\beta}})$ e $\gamma^{P E}$ é dada por

$$
\mathrm{B}(\hat{\boldsymbol{\beta}})=-\frac{\phi}{8 d_{g} \rho^{2}} \mathbf{L}\left(\sum_{j=1}^{p} \mathbf{a}_{j j}^{P E}\right) .
$$

Prova. Seja a decomposição QR de

$$
\mathbf{V}^{T} \mathbf{V}=\mathbf{R}^{T} \mathbf{Q}^{T} \mathbf{Q R}=\mathbf{R}^{T} \mathbf{R}=\tilde{\mathbf{R}}^{T} \tilde{\mathbf{R}}=\left(\mathbf{L L}^{T}\right)^{-1}
$$

e como $\left(\mathbf{D}_{\beta}^{T} \mathbf{D}_{\beta}\right)=\rho^{2}\left(\mathbf{V}^{T} \mathbf{V}.\right)$ e $\mathbf{D}_{\beta \beta}(i)=\rho \mathbf{V}_{\text {.. }}(i)$, temos que

$$
\begin{aligned}
\operatorname{tr}\left\{\left(\mathbf{D}_{\beta}^{T} \mathbf{D}_{\beta}\right)^{-1} \mathbf{D}_{\beta \beta}(i)\right\} & =\operatorname{tr}\left\{\mathbf{D}_{\beta \beta}(i)\left(\mathbf{D}_{\beta}^{T} \mathbf{D}_{\beta}\right)^{-1}\right\}=\operatorname{tr}\left\{\mathbf{D}_{\beta \beta}(i)\left(\mathbf{L} \mathbf{L}^{T}\right)\right\} / \rho^{2} \\
& \left.=\operatorname{tr}\left\{\mathbf{L}^{T} \mathbf{D}_{\beta \beta}(i)\right\} \mathbf{L}\right\} / \rho^{2}=\operatorname{tr}\{\mathbf{U}(i)\} / \rho \\
& =\sum_{j=1}^{p} U_{i j j} / \rho
\end{aligned}
$$

Substituindo (A.6) em (A.5) então $\mathrm{B}(\hat{\beta})$ pode ser reescrito em função da curvatura paramétrica dada abaixo

$$
\begin{aligned}
\mathbf{B}(\hat{\boldsymbol{\beta}}) & =-\frac{\phi}{8 d_{g} \rho^{2}} \mathbf{L}\left[\mathbf{L}^{T} \mathbf{D}_{\beta}^{T}\right]\left[\sum_{j=1}^{p} \mathbf{U}_{j j} / \rho\right]=-\frac{\phi}{8 d_{g} \rho^{2}} \mathbf{L}\left[\mathbf{L}^{T} \mathbf{V}_{.}^{T}\right]\left[\sum_{j=1}^{p} \mathbf{U}_{j j}\right] \\
& =-\frac{\phi}{8 d_{g} \rho^{2}} \mathbf{L}\left[\mathbf{L}^{T}(\mathbf{Q R})^{T}\right]\left[\sum_{j=1}^{p} \mathbf{U}_{j j}\right]=-\frac{\phi}{8 d_{g} \rho^{2}} \mathbf{L}\left[\left(\mathbf{Q R} \tilde{\mathbf{R}}^{-1}\right)^{T}\right]\left[\sum_{j=1}^{p} \mathbf{U}_{j j}\right] \\
& =-\frac{\phi}{8 d_{g} \rho^{2}} \mathbf{L}\left[\mathbf{Q}^{T}\right]\left[\sum_{j=1}^{p} \mathbf{U}_{j j}\right]=-\frac{\phi}{8 d_{g} \rho^{2}} \mathbf{L}\left(\sum_{j=1}^{p} \mathbf{a}_{j j}^{P E}\right) .
\end{aligned}
$$

em que Q. é uma matriz formada das primeiras linha de $\mathrm{Q}$.

Como o viés está relacionado com a medida de não-linearidade $\gamma^{P E}$, podemos reduzí-lo através de reparametrizações no modelo e a expressão (A.5) pode indicar quais parâmetros são os possíveis responsáveis pelo alto valor da medida de nãolinearidade. 


\section{APENNDICE B}

\section{Probabilidades de Nível}

\section{B.1 Caso de $k=2$ restrições}

Para o caso de $k=2$ restrições os pesos ficam dados por (vide, por exemplo, Wolak, 1987)

$$
\begin{aligned}
\omega(2,0, \Delta) & =\frac{1}{2} \pi^{-1} \arccos \left(\rho_{12}\right) ; \\
\omega(2,1, \Delta) & =\frac{1}{2} \mathrm{e} \\
\omega(2,2, \Delta) & =\frac{1}{2}-\omega(2,0, \Delta),
\end{aligned}
$$

em que $\rho_{i j}$ denota o elemento $(i, j)$ da matriz de correlações lineares associadas à matriz $\Delta$.

\section{B.2 Caso de $k=3$ restrições}

Para o caso de $k=3$ restrições os pesos ficam dados por (vide, por exemplo, Wolak, 1987)

$$
\begin{aligned}
\omega(3,0, \Delta) & =\frac{1}{2}-\omega(3,2, \Delta) ; \\
\omega(3,1, \Delta) & =\frac{1}{2}-\omega(3,3, \Delta) ; \\
\omega(3,2, \Delta) & =\frac{1}{4} \pi^{-1}\left\{3 \pi-\arccos \left(\rho_{12.3}\right)-\arccos \left(\rho_{13.2}\right)-\arccos \left(\rho_{23.1}\right)\right\} \mathrm{e} \\
\omega(3,3, \Delta) & =\frac{1}{4} \pi^{-1}\left\{2 \pi-\arccos \left(\rho_{12}\right)-\arccos \left(\rho_{13}\right)-\arccos \left(\rho_{23}\right)\right\},
\end{aligned}
$$

em que $\rho_{i j . t}$ 's correspondem aos coeficientes de correlação linear parcial, os quais são definidos por

$$
\rho_{i j . t}=\frac{\rho_{i j}-\rho_{i t} \rho_{j t}}{\sqrt{\left(1-\rho_{i t}^{2}\right)\left(1-\rho_{j t}^{2}\right)}} .
$$




\section{B.3 Caso de $k=4$ restrições}

No caso de $k=4$ restrições temos as expressões abaixo para os pesos (vide, por exemplo, Wolak 1987)

$$
\begin{aligned}
\omega(4,0, \Delta)= & \frac{1}{2}-\omega(4,4, \Delta)-\omega(4,2, \Delta) \\
\omega(4,1, \Delta)= & -\frac{1}{2}+\frac{1}{8 \pi} \sum_{i>j ; i, j \neq k} \arccos \left(\rho_{i j . k}\right) ; \\
\omega(4,2, \Delta)= & \frac{1}{4 \pi^{2}} \sum_{i>j, k>\ell ; \ell \neq i, j} \arccos \left(\rho_{i j}\right)\left\{\pi-\arccos \left(\rho_{k \ell . i j}\right)\right\} ; \\
\omega(4,3, \Delta)= & \frac{1}{2}-\omega(4,1, \Delta) \mathrm{e} \\
\omega(4,4, \Delta)= & \frac{1}{16}+\frac{1}{8 \pi}\left\{\operatorname{arcsen}\left(\rho_{12}\right)+\operatorname{arcsen}\left(\rho_{13}\right)+\operatorname{arcsen}\left(\rho_{14}\right)\right. \\
& \left.+\operatorname{arcsen}\left(\rho_{23}\right)+\operatorname{arcsen}\left(\rho_{24}\right)+\operatorname{arcsen}\left(\rho_{34}\right)\right\}+\frac{1}{4 \pi^{2}} \eta,
\end{aligned}
$$

em que $\eta$ (vide, Childs, 1967; Sun, 1988a) é dado por

$$
\eta=\int_{0}^{1} \sum_{k=2}^{4} \frac{\rho_{1 k}}{\sqrt{1-\rho_{1 k}^{2} t^{2}}} I_{2}\left(R_{2}^{1, k}\right) d t
$$

com $I_{2}\left(R_{2}^{1,2}\right), I_{2}\left(R_{2}^{1,3}\right)$ e $I_{2}\left(R_{2}^{1,4}\right)$ sendo

$$
\begin{aligned}
& I_{2}\left(R_{2}^{1,2}\right)=\operatorname{arcsen}\left(\frac{r_{34}^{12}(t)}{\sqrt{r_{33}^{12}(t) r_{44}^{12}(t)}}\right) \\
& I_{2}\left(R_{2}^{1,3}\right)=\operatorname{arcsen}\left(\frac{r_{24}^{13}(t)}{\sqrt{r_{22}^{13}(t) r_{44}^{13}(t)}}\right) \mathrm{e} \\
& I_{2}\left(R_{2}^{1,4}\right)=\operatorname{arcsen}\left(\frac{r_{23}^{14}(t)}{\sqrt{r_{22}^{14}(t) r_{33}^{14}(t)}}\right)
\end{aligned}
$$

em que as entradas da matriz $2 \times 2 R_{2}^{1, k}$ são dadas por

$$
\begin{aligned}
r_{i j}^{1 k}(t)= & \left\{\rho_{i j}-\rho_{k i} \rho_{k j}-t^{2}\left(\rho_{1 k}^{2} \rho_{i j}+\rho_{1 i} \rho_{1 j}\right.\right. \\
& \left.\left.-\rho_{1 k} \rho_{1 i} \rho_{k j}-\rho_{1 k} \rho_{1 j} \rho_{k i}\right)\right\},
\end{aligned}
$$

$i, j, k=1,2,3$. A correlação parcial $\rho_{k \ell \ell i j}$ é definida abaixo

$$
\rho_{k \ell . i j}=\frac{\rho_{k \ell . i}-\rho_{k j . i} \rho_{\ell j . i}}{\sqrt{\left(1-\rho_{k j . i}^{2}\right)\left(1-\rho_{\ell j . i}^{2}\right)}} .
$$




\section{APÊNDICE C}

\section{Coelhos}

Tabela C.1 Pesos das lentes dos olhos de coelhos europeus (y), em miligramas, a idade (x) em dias numa amostra de 71 observações.

\begin{tabular}{rr|cc}
\hline \multicolumn{1}{c}{$x$} & \multicolumn{1}{c|}{$y$} & $x$ & $y$ \\
\hline 15 & 21,66 & 195 & 161,10 \\
15 & 22,75 & 218 & 174,18 \\
15 & 22,30 & 218 & 173,03 \\
18 & 31,25 & 219 & 173,54 \\
28 & 44,79 & 224 & 178,86 \\
29 & 40,55 & 225 & 177,68 \\
37 & 50,25 & 227 & 173,73 \\
37 & 46,88 & 232 & 159,98 \\
44 & 52,03 & 232 & 161,29 \\
50 & 63,47 & 237 & 187,07 \\
50 & 61,13 & 246 & 176,13 \\
60 & 81,00 & 258 & 183,40 \\
61 & 73,09 & 276 & 186,26 \\
64 & 79,09 & 285 & 189,66 \\
65 & 79,51 & 300 & 186,09 \\
65 & 65,31 & 301 & 186,70 \\
72 & 71,90 & 305 & 186,80 \\
75 & 86,10 & 312 & 195,10 \\
75 & 94,60 & 317 & 216,41 \\
82 & 92,50 & 338 & 203,23 \\
85 & 105,00 & 347 & 188,38 \\
91 & 101,70 & 354 & 189,70 \\
91 & 102,90 & 357 & 195,31 \\
97 & 110,00 & 375 & 202,63 \\
98 & 104,30 & 394 & 224,82 \\
125 & 134,90 & 513 & 203,30 \\
142 & 130,68 & 535 & 209,70 \\
142 & 140,58 & 554 & 233,90 \\
147 & 155,30 & 591 & 234,70 \\
147 & 152,20 & 648 & 244,30 \\
150 & 144,50 & 660 & 231,00 \\
159 & 142,15 & 705 & 242,40 \\
165 & 139,81 & 723 & 230,77 \\
183 & 153,22 & 756 & 242,57 \\
192 & 145,72 & 768 & 232,12 \\
& & 860 & 246,70 \\
\hline & & &
\end{tabular}


APÊNDICE D

\section{Estoque}

Tabela D.1 Tempo gasto no serviço (y) em minutos, número de bebidas estocadas $\left(x_{1}\right)$ e distância percorrida $\left(x_{2}\right)$ em pés numa amostra de 25 observações.

\begin{tabular}{rrr}
\hline \multicolumn{1}{c}{$y$} & $x_{1}$ & \multicolumn{1}{c}{$x_{2}$} \\
\hline 16,68 & 7 & 560 \\
11,50 & 3 & 220 \\
12,03 & 3 & 340 \\
14,88 & 4 & 80 \\
13,75 & 6 & 150 \\
18,11 & 7 & 330 \\
8,00 & 2 & 110 \\
17,83 & 7 & 210 \\
79,24 & 30 & 1460 \\
21,50 & 5 & 605 \\
40,33 & 16 & 688 \\
21,00 & 10 & 215 \\
13,50 & 4 & 255 \\
19,75 & 6 & 462 \\
24,00 & 9 & 448 \\
29,00 & 10 & 776 \\
15,35 & 6 & 200 \\
19,00 & 7 & 132 \\
9,50 & 3 & 36 \\
35,10 & 17 & 770 \\
17,90 & 10 & 140 \\
52,32 & 26 & 810 \\
18,75 & 9 & 450 \\
19,83 & 8 & 635 \\
10,75 & 4 & 150 \\
\hline & &
\end{tabular}


APÊNDICE E

\section{TV a cabo}

Tabela E.1 Conjuntos de dados sobre demanda de TV a cabo.

\begin{tabular}{rrrrrrr}
\hline$y$ & $x_{1}$ & $x_{2}$ & $x_{3}$ & $x_{4}$ & $x_{5}$ & $x_{6}$ \\
\hline 105 & 350 & 9839 & 14,95 & 10 & 16 & 13 \\
90 & 255,631 & 10606 & 15 & 7,5 & 15 & 11 \\
14 & 31 & 10455 & 15 & 7 & 11 & 9 \\
11,7 & 34,840 & 8958 & 10 & 7 & 22 & 10 \\
46 & 153,434 & 11741 & 25 & 10 & 20 & 12 \\
11,217 & 26,621 & 9378 & 15 & 7,66 & 18 & 8 \\
12 & 18 & 10433 & 15 & 7,5 & 12 & 8 \\
6,428 & 9,324 & 10167 & 15 & 7 & 17 & 7 \\
20,1 & 32 & 9218 & 10 & 5,6 & 10 & 8 \\
8,5 & 28 & 10519 & 15 & 6,5 & 6 & 6 \\
1,6 & 8 & 10025 & 17,5 & 7,5 & 8 & 6 \\
1,1 & 5 & 9714 & 15 & 8,95 & 9 & 9 \\
4,355 & 15,204 & 9294 & 10 & 7 & 7 & 7 \\
78,910 & 97,889 & 9784 & 24,95 & 9,49 & 12 & 7 \\
19,6 & 93 & 8173 & 20 & 7,5 & 9 & 7 \\
1 & 3 & 8967 & 9,95 & 10 & 13 & 6 \\
1,65 & 2,6 & 10133 & 25 & 7,55 & 6 & 5 \\
13,4 & 18,284 & 9361 & 15,5 & 6,3 & 11 & 5 \\
18,708 & 55 & 9085 & 15 & 7 & 16 & 6 \\
1,352 & 1,7 & 10067 & 20 & 5,6 & 6 & 6 \\
170 & 270 & 8908 & 15 & 8,75 & 15 & 5 \\
15,388 & 46,540 & 9632 & 15 & 8,73 & 9 & 6 \\
6,555 & 20,417 & 8995 & 5,95 & 5,95 & 10 & 6 \\
40 & 120 & 7787 & 25 & 6,5 & 10 & 5 \\
19,9 & 46,39 & 8890 & 15 & 7,5 & 9 & 7 \\
2,45 & 14,5 & 8041 & 9,95 & 6,25 & 6 & 4 \\
3,762 & 9,5 & 8605 & 20 & 6,5 & 6 & 5 \\
24,882 & 81,98 & 8639 & 18 & 7,5 & 8 & 4 \\
21,187 & 39,7 & 8781 & 20 & 6 & 9 & 4 \\
3,487 & 4,113 & 8551 & 10 & 6,85 & 11 & 4 \\
3 & 8 & 9306 & 10 & 7,95 & 9 & 6 \\
42,1 & 99,750 & 8346 & 9,95 & 5,73 & 8 & 5 \\
20,350 & 33,379 & 8803 & 15 & 7,5 & 8 & 4 \\
23,15 & 35,5 & 8942 & 17,5 & 6,5 & 8 & 5 \\
9,866 & 34,775 & 8591 & 15 & 8,25 & 11 & 4 \\
42,608 & 64,840 & 9163 & 10 & 6 & 11 & 6 \\
10,371 & 30,556 & 7683 & 20 & 7,5 & 8 & 6 \\
5,164 & 16,5 & 7924 & 14,95 & 6,95 & 8 & 5 \\
31,150 & 70,515 & 8454 & 9,95 & 7 & 10 & 4 \\
18,350 & 42,040 & 8429 & 20 & 7 & 6 & 4 \\
\hline & & & & & &
\end{tabular}


APÊNDICE F

\section{Pacientes diabéticos}

Tabela F.1 Efeito de um teste físico em pacientes hospitalares.

\begin{tabular}{|c|c|c|c|c|c|c|c|c|c|}
\hline \multirow[b]{2}{*}{ Sujeito } & & \multicolumn{8}{|c|}{ Tempo (em minutos) } \\
\hline & & 1 & 2 & 3 & 4 & 5 & 6 & 8 & 10 \\
\hline \multirow[t]{8}{*}{ Grupo 1} & 1 & 7,6 & 7,5 & 8,9 & 9,5 & 8,7 & 8,8 & * & 7,0 \\
\hline & 2 & 10,1 & 10,4 & 10,4 & 8,9 & 8,9 & 8,4 & 9,9 & 8,6 \\
\hline & 3 & 11,2 & 12,8 & 10,0 & 10,3 & 9,5 & 9,2 & 9,0 & 9,4 \\
\hline & 4 & 10,8 & 10,3 & 9,3 & 10,3 & 11,5 & 12,3 & 10,0 & 11,4 \\
\hline & 5 & 3,9 & 3,9 & 4,5 & 3,2 & 4,1 & 4,0 & 3,5 & 3,7 \\
\hline & 6 & 6,7 & 7,0 & 7,9 & 7,4 & 7,3 & 7,2 & 6,6 & 6,6 \\
\hline & 7 & 2,2 & 2,0 & 2,2 & 2,2 & 2,5 & 2,3 & 2,5 & 2,4 \\
\hline & 8 & 2,1 & 2,4 & 2,5 & 2,3 & 2,0 & 2,0 & 1,9 & 2,0 \\
\hline \multirow[t]{6}{*}{ Grupo 2} & 9 & 8,5 & 8,4 & 8,5 & 8,2 & 5,6 & 8,8 & 8,8 & 8,4 \\
\hline & 10 & 7,5 & 7,1 & 7,2 & 7,0 & 5,0 & 4,2 & 6,9 & 9,5 \\
\hline & 11 & 12,9 & 13,5 & 13,4 & 13,1 & 13,6 & 13,1 & 14,8 & 15,3 \\
\hline & 12 & 8,8 & 9,2 & 8,4 & 9,2 & 7,9 & 7,9 & 7,9 & 7,3 \\
\hline & 13 & 5,5 & 5,6 & 5,2 & 5,3 & 6,4 & 6,0 & 6,4 & 6,4 \\
\hline & 14 & 3,2 & 4,0 & 3,2 & 3,4 & 3,4 & 3,2 & 3,2 & 3,2 \\
\hline \multirow[t]{7}{*}{ Grupo 3} & 15 & 5,5 & 5,5 & 5,3 & 5,0 & 4,5 & 4,1 & 4,3 & 3,9 \\
\hline & 16 & 0,4 & 0,6 & 0,4 & 0,4 & 0,5 & 0,6 & 0,5 & 0,5 \\
\hline & 17 & 6,2 & 6,3 & 6,6 & 5,9 & 6,5 & 5,5 & 5,7 & 5,1 \\
\hline & 18 & 4,6 & 3,8 & 3,9 & 3,6 & 3,0 & 3,7 & 3,2 & 3,1 \\
\hline & 19 & 3,2 & 3,2 & 2,7 & 2,7 & 2,4 & 2,2 & 1,8 & 1,7 \\
\hline & 20 & 10,8 & 8,7 & 9,3 & 10,5 & 12,7 & 11,3 & 19,1 & 18,9 \\
\hline & 21 & 5,7 & 7,0 & 7,0 & 5,8 & 6,9 & 7,7 & 7,5 & 8,8 \\
\hline
\end{tabular}




\section{Referências}

Albert, J.; Delampady, M. e Polasek, W. (1991). A class of distribution for robustness studies. Journal of Statistical Planning and Inference, 28, 291-304.

Anderson, T.W. e Fang, K.T (1987). Cochran's theorem for elliptically contourned distributions. Sankhya A, 49, 305-315.

Arellano-Valle, R.B. (1994). Elliptical Distribution: Properties and Applications in Regression Models. Tese de doutorado, Departamento de Estatística, Universidade de São Paulo, Brasil.

Ascombe, F. (1961). Examination of residuals In Procedings 4th Berkeley Symposium, 1, 1-36.

Aitkin, M. (1987). Modelling variance heterogeneity in normal regression using GLIM. Applied Statistics, 36, 332-339.

Atkinson, A.C. (1981). Two graphical display for outlying and influential observations in regression. Biometrika, 68, 13-20.

Atkinson, A.C. (1985). Plots, Transformation and Regression. Clarendon Press : Oxford.

Barlow, R.E.; Bartholomew, D.J.; Bremmer, J.N. e Brunk, H. H. (1972). Statistical Inference under Order Restrictions. New York: John Wiley.

Barroso, L.P.; Cordeiro, G.M. e Vasconcellos, K.L.P. (2002). Second-Order Asymptotic for Score Tests in Heteroskedastic t Regression Models. Communications in Statistics - Theory and Methods, 31, 1515-1529.

Bartholomew, D.J. (1959a). A test of homogeneity for ordered alternatives, I. Biometrika, 46, 36-48.

Bartholomew, D.J. (1959b). A test of homogeneity for ordered alternatives, II. Biometrika, 46, 328-335. 
Bartholomew, D.J. (1961). A test of homogeneity of means under restricted alternatives. Journal of the Royal Statistical Society B, 23, 239-281.

Bates, D.M. e Watts, D.G. (1980). Relative curvature of nonlinearity. Journal of the Royal Statistical Society B, 42, 1-25.

Bates, D.M. e Watts, D.G. (1988). Nonlinear Regression Analysis and its Applications. New York: John Wiley.

Beale, E.M.L. (1960). Confidence region in nonlinear estimation. Journal of the Royal Statistical Society B, 22, 41-76.

Becker, R.A.; Chambers, J.M. e Wilks, A.R. (1988). The New S Language. New York: Chapman and Hall.

Berkane, M. e Bentler, P.M. (1986). Moments of elliptical distributed random variates. Statistics and Probability Letters, 4, 333-335.

Bickel, P. (1978). Using residuals robustly I:Tests for heteroscedasticity, nonlinearity. The Annals of Statistics, 6, 266-291.

Bohrer, R. e Chow, W. (1978). Algorithm AS122. Weights for one-sided multivariate inference. Applied Statistics, 27, 100-104.

Box, M.J. (1971). Bias in non-linear estimation (with discussion). Journal of the Royal Statistical Society B, 33, 171-201.

Box, M.J. e Tiao, G.C. (1973). Bayesian Inference in Statistical Analysis. London: Addison-Wesley.

Businger, P. e Golub, G.H. (1965). Least squares by Householder transformations. Numerische Math., 7, 269-276.

Cambanis. S; Huang, S. e Simons, G. (1981). On the theory of elliptically contoured distributions. Journal of Multivariate Analysis, 11, 368-385.

Cardoso-Neto, J. e Paula, G.A. (2001). Wald one-sided test using generalized estimating equations. Computational Statistics and Data Analysis, 36, 475-495.

Carroll, R.J. e Ruppert, D. (1988). Transformation and Weighting in Regression. New York: Chapman and Hall.

Chambers, J.M. e Hastie, T.J. (eds) (1992). Statistical Models in S. New York : Chapman and Hall. 
Childs, D.P. (1967). Reduction of the multivariate normal integral to characteristic form. Biometrika, 54, 293-300.

Chmielewski, M.A. (1981). Elliptically symmetric distributions: a review and bibliography. International Statistical Review, 49, 67-74.

Cook, R. D. (1986). Assessment of local influence (with discussion). Journal of the Royal Statistical Society B, 48, 133-169.

Cook, R. D. e Weisberg, S. (1982). Residuals e Influence in Regression. New York: Chapman and Hall.

Cook, R.D. e Weisberg, S. (1983). Diagnostics for heteroscedasticity in regression. Biometrika, 70, 1-10.

Cook, R.D. e Tsai, C.L. (1985). Residuals in nonlinear regression. Biometrika, 72, 23-29.

Cook, R.D.; Tsai, C.L. e Wei, B.C. (1986). Bias in nonlinear regression. Biometrika, 73, 615-623.

Cordeiro, G.M. (2004). Corrected LR tests in symmetric nonlinear regression models. Journal Statistical Computation and Simulation, aceito para publicação.

Cordeiro, G.M.; Ferrari, S.L.P.; Uribe-Opazo, M.A. e Vasconcellos, K.L.P. (2000). Corrected maximum likelihood estimation in a class of symmetric nonlinear regression models. Statistics and Probability Letters, 46, 317-328.

Cordeiro, G.M. e McCullagh, P. (1991). Bias correction in generalized linear models. Journal of the Royal Statistical Society B, 53, 629-643.

Cox, D.R. e Hinkley, D.V. (1974). Theoretical Statistics. London: Chapman and Hall.

Cox, D.R. e Snell, E.J. (1968). A general definition of residuals Journal of the Royal Statistical Society B, 30, 248-275.

Crowder, M.J. e Hand, D.J. (1990). Analysis of Repeated Measures. London: Chapman and Hall.

Cysneiros, F.J.A. e Paula, G.A. (2003). One-sided tests in univariate elliptical linear regression models. In: Proceedings of the 18th International Workshop on Statistical Modelling, Verbeke, G., Molenberghs, G., Aerts, A. and Fieuws, S. 
(Eds.). Leuven: Katholieke Universiteit Leuven, pp. 103-108.

Cysneiros, F.J.A. e Paula, G.A. (2004). One-sided test in linear models with multivariate $t$-distribution. Communications in Statistics-Simulation and Computation, 33, aceito para publicação.

Devlin, S.J.; Gnanadesikan, R. e Kettenring, J.R. (1976). Some multivariate applications of elliptical distributions. Essays in Probability and Statistics.

Devroye, L. (1986). Non-Uniform Random Variable Generator. New York: Springer-Verkag.

Dickey, J.M. (1967). Multivariate generalizations of the multivariate $t$ distribution and the inverted multivariate $t$ distribution. Annals of Mathmatical Statistics, $38,511-518$.

Doornik, J.A. (1999). Object-Oriented matrix programming using Ox, 3rd ed. London: Timberlake Consultants Press and Oxford: www.nuff.ox.ac.uk/Users/Doornik.

Emerson, J.D.; Hoaglin, D.C. e Kempthorne, P.J. (1984). Leverage in least squares additive-plus-multiplicative fits for two-way tables. Journal of the American Statistical Association, 79, 329-335.

Escobar, L.A. e Meeker, W.Q. (1992). Assessing influence in regression analysis with censored data. Biometrics, 48, 507-528.

Fahrmeir, L. e Klinger, J. (1994). Estimating and testing generalized linear models under inequality restrictions. Statistical Papers, 35, 211-229.

Fang, K.T. e Anderson, T.W. (1990). Statistical Inference in Elliptical Contoured and Related Distributions. New York: Allerton Press.

Fang, K.T. e Zhang, Y.T. (1990). Generalized Multivariate Analysis. New York: Springer-Verlag.

Fang, K.T.; Kotz, S. e Ng, K.W. (1990). Symmetric Multivariate and Related Distributions. London: Chapman and Hall.

Ferrari, S.L.P e Arellano-Valle, R.B. (1996). Bartlett corrected tests for regression models with Student- $t$ independent errors . Brazilian Journal of Probability and Statistics, 10, 15-33. 
Ferrari, S.L.P; Cysneiros, A.H.M.A. e Cribari-Neto, F. (2004). An improved test for heterokedasticity using adjusted modified profile likelihood inference. Journal of Statistical Planning and Inference, aceito para publicação.

Ferrari, S.L.P. e Uribe-Opazo, M.A. (2001). Corrected likelihood ratio tests in class of symmetric linear Regression models. Brazilian Journal of Probability and Statistics, 15, 49-67.

Fernandez, C. e Steel, M.F.J. (1999). Multivariate student-t regression models: Pitfalls and inference. Biometrika, 86, 153-167.

Fiacco, A.V. e McCormick, G.P. (1968). Nonlinear Programming : Sequential Unconstrained Minimization Techniques. New York : John Wiley.

Galea, M.; Bolfarine, H. e Vilca-Labra, F. (2002). Influence diagnostics for the structural error-in-variables model under the Student- $t$ distribution. Journal of Applied Statistics, 29, 1191-1204.

Galea, M.; Paula, G.A. e Bolfarine, H. (1997). Local influence in elliptical linear regression models. The Statistician, 46, 71-79.

Galea, M.; Paula, G.A. e Uribe-Opazo, M. (2003). On influence diagnostic in univariate elliptical linear regression models. Statistical Papers, 44, 23-45.

Gouriéroux, C.; Holly, A. e Monfort, A. (1982). Likelihood ratio test, Wald test, and Kuhn-Tucker test in linear models with inequality constraints on the regression parameters. Econometrica, 50, 63-80.

Gouriéroux, G. e Monfort, A. (1995). Statistics and Econometric. Vols. 1 e 2. Cambridge: Cambridge University Press.

Gumbel, E. (1944). Ranges and midranges. Annals of Mathematical Statistics, 15, 414-422.

Gupta, A. K. e Varga, T. (1993). Elliptically Contoured Models in Statistics. Kluwer Academic Publishers.

Hastings, N.A.J. e Peacock, J.B. (1975). Statistical Distributions. New York: John Wiley.

Harvey, A.C. (1976). Estimating regression models with multiplicative heteroscedasticity. Econometrica, 41, 461-465. 
Hildreth, C. (1957). A quadratic programming procedure. Naval Research Logistics Quartely, 4, 79-85.

Hoaglin, D.C. e Welsch, R.E. (1978). The hat matrix in regression and ANOVA. The American Statistician, 32, 17-22.

Ihaka, R. e Gentleman, R. (1996). R: A language for data analysis and graphics. Journal of Computational Graphics and Statistics, 5, 299-314.

Johnson, R. e Kotz, S. (1970). Continuous Univariate Distributions v.2. Boston: Houghton Mifflin.

Kelker, D. (1970). Distribution theory of spherical distributions and a locationscale parameter generalization. Sankhya A, 32, 419-430.

Kodde, D.A. e Palm, F.C. (1986). Wald criteria for jointly testing equality and inequality restrictions. Econometrica, 54, 1243-1248.

Kotz S. (1975). Multivariate distributions at a cross-road. Statiscal Distributions in Scientific Work, 1 Ed. GP. Patil, S. Kotz e J.K. Ord., 247-270. Dordrecht, Reiden.

Kowalski, J.; Mendoza-Blanco, J.; Tu, X.M. e Gleser,L.J. (1999). On the difference in inference and prediction between the joint and independent $t$-error models for seemingly unrelated regressions. Communications in Statistics, Theory and Methods, 28, 2119-2140.

Kudô, A. (1963). A multivariate analogue of the one-sided test. Biometrika 50, 403-418.

Lange, K.L.; Little, R.J.A. e Taylor, J.M.G. (1989). Robust statistical modeling using the $t$ distribution. Journal of the American Statistical Association, 84, 881-896.

Lee, C.C.; Robertson, T. e Wright, F.T. (1993). Bounds on distributions arising in order restricted inferences with restricted weights. Biometrika, 80, 405-416.

Lesaffre, F. e Verbeke, G. (1998). Local influence in linear mixed models. Biometrics, 38, 963-974.

Little, R.J.A. (1988). Robust estimation of the mean and covariance matrix from data with missing values Applied Statistics, 37, 23-39. 
Luenberger, D.G. (1969). Optimization by Vector Space Methods. New York: John Wiley.

Manoukian, E.B. (1985). Modern Concepts and Theorems of Mathematical Statistics. New York: Springer-Verlag.

Maronna, R.A. (1976). Robust M-estimators of multivariate location and scatter. The Annals of Statistics, 4, 51-67.

Montgomery, D.C.; Peck, E.A. e Vining, G.G. (2001). Introduction to Linear Regression Analysis, 3rd ed. New York: John Wiley.

Muirhead, R. (1980). The effects of symmetric distributions on some standard procedures involving correlation coefficients. In Multivariate Statistical Analysis (ed. R.P. Gupta) North-Holland, 143-159.

Muirhead, R. (1982). Aspects of Multivariate Statistical Theory. New York: John Wiley.

Nocedal, J. e Wright, S.J. (1999). Numerical Optimization. New York: SpringerVerlag.

Nüesch, P.E. (1964). Multivariate Test of Location for Restricted Alternatives. Tese de doutorado - Swiss Federal Institute of Technology, Zurich.

Nüesch, P.E. (1966). On the problem of testing location in multivariate populations for restricted alternatives. Annals of Mathematical Statistics, 37, 113-119.

Nyquist, H. (1991). Restricted estimation of generalized linear models. Applied Statistics, 40, 133-141.

Park, R.E. (1966). Estimating with heteroscedastic error terms. Econometrica, 34, 888.

Park, T.P.; Shin, D. W. e Park, C.G. (1998). A generalized estimating equations approach for testing ordered group effects with repeated measurements. Biometrics, 54, 1645-1653.

Paula, G. A. (1992). Bias correction for exponential family nonlinear models. Journal of Statistical Computation and Simulation, 40, 43-54.

Paula, G.A. (1993). Assessing local influence in restricted regression models. Computational Statistics and Data Analysis, 16, 63-79. 
Paula, G.A. (1995). Influence and residuals in restricted generalized linear models. Journal of Statistical Computation and Simulation, 51, 315-331.

Paula, G.A. (1996). On approximation of the level probabilities for testing ordered parallel regression lines. Statistics and Probability Letters, 30, 333-338.

Paula, G.A. (1997). Estimação e Testes em Modelos de regressão com Parâmetros Restritos. Livro texto de minicurso da 5a. Escola de Modelos de Regressão, Campos do Jordão, SP.

Paula, G. A. (1999a). One-sided test in generalized linear dose-response models Computational Statistics and Data Analysis, 30, 413-427.

Paula, G.A. (1999b). Leverage in inequality constrained regression models. The Statistician, 48, 529-538.

Paula, G.A. e Artes, R. (2000). One-sided test to assess correlation in logistic linear models using estimating equations. Biometrical Journal, 42, 701-714.

Paula, G.A., Cysneiros, F.J.A. e Galea, M. (2003). Local influence and leverage in elliptical nonlinear regression models. In: Proceedings of the 18th International Workshop on Statistical Modelling, Verbeke, G., Molenberghs, G., Aerts, A. and Fieuws, S. (Eds.). Leuven: Katholieke Universiteit Leuven, pp. 361-365.

Paula, G.A. e Rojas, O.V. (1997). On restricted hypotheses in extreme value regression models. Computational Statistics and Data Analysis, 25, 143-157.

Paula, G.A. e Sen, P.K. (1994). Tests of ordered hypotheses in linkage in heredity. Statistics and Probability Letters, 20, 395-400.

Paula, G.A. e Sen, P.K. (1995). One-sided tests in generalized linear models with parallel regression lines. Biometrics, 51, 1494-1501.

Perlman, M.D. (1969). One-sided problems in multivariate analysis. Annals of Mathematical Statistics, 40, 549-567.

Piegorch, W. (1990). One-sided-significance tests for generalized linear models under dichotomous response. Biometrics, 46, 309-316.

Pinheiro, J.C.; Liu, C. e Wu, Y.N. (2001). Efficient Algorithms for robust estimation in linear mixed-effects models using the multivariate t distribution. Journal of Computation and Graphical Statistics, 10, 249-276. 
Ramanathan, R. (1993). Statistical Methods in Econometrics. New York: John Wiley.

Rao, B.L.S.P. (1990). Remarks on univariate symmetric distributions. Statistics and Probability Letters, 10, 307-315.

Ratkowsky, D.A. (1983). Nonlinear Regression Modelling. Marcel Dekker: New York.

Robertson, T.; Wright, F.T. e Dykstra, R.L. (1988). Order Restricted Statistical Inference. New York: John Wiley.

Ryan, D.M. (1974). Penalty and barrier functions. In Numerical Methods for Constrained Optimization (Eds. P.E. Gill and W. Murray), pp. 175-190. New York: Academic Press.

Seber, G.A. e Wild, C.J. (1989). Nonlinear Regression. New York : John Wiley.

Serfling, R.J. (1980). Approximation Theorems of Mathematical Statistics. New York: John Wiley.

Sen, P.K. e Silvapulle, M.J. (2002). An appraisal of some aspects of statistical inference under inequality constraints. Journal of Statistical Planning and Inference, $107,3-44$.

Shapiro, A. (1985). Asymptotic distribution of test statistics in the analysis of moment structures under inequality constraints. Biometrika, 72, 133-144.

Shapiro, A. (1988). Towards a unified theory of inequality constrained testing in multivariate analysis. International Statistical Review, 56, 49-62.

Shin, D.W.; Park, C.G. e Park, T.P. (1996). Testing for ordered group effects with repeated measurements. Biometrika, 83, 688-694.

Silvapulle, M.J. (1991). On limited dependent variable models: maximum likelihood estimation and test of one-sided hypothesis. Econometric Theory, 7, 385-395.

Silvapulle, M.J. (1994). On tests against one-sided hypotheses in some generalized linear models. Biometrics, 50, 853-858.

Silvapulle, M.J. e Silvapulle, P. (1995). A score test against one-sided alternative. Journal of the American Statistical Association, 90, 342-349.

Smyth, G.K. (1989). Generalized linear models with varying dispersion. Journal of 
in the linear regression model. Journal of the American Statistical Association, 82, 782-793.

Wolak, F.A. (1989a). Testing inequality constraints in linear econometric models. Journal of Econometrics, 41, 205-235.

Wolak, F.A. (1989b). Local and global testing of linear and nonlinear inequality constraints in nonlinear econometric models. Econometric Theory, 5, 1-35.

Wolak, F.A. (1991). The local nature of hypothesis tests involving inequality constraints in nonlinear models., Econometrica 59, 981-995.

Yamaguchi, K. (1990). Generalized EM algorithm for model with contaminated error term. In Proceedings of the Seven Japan and Korea Joint Conference of Statistics, 107-114 